\title{
Development of Tunable Diode Laser Absorption Tomography and Application to Scramjet Engines
}

\author{
A Dissertation \\ Presented to \\ the faculty of the School of Engineering and Applied Science \\ University of Virginia
}

\begin{abstract}
in partial fulfillment
of the requirements for the degree of

Doctor of Philosophy

Mechanical and Aerospace Engineering
\end{abstract}

by

Kristin Michelle Busa

August

2014 
APPROVAL SHEET

This dissertation is submitted in partial fulfillment of the requirements for the degree of

Doctor of Philosophy in Mechanical and Aerospace Engineering

Kristin M. Busa, Author

This dissertation has been read and approved by the Examining Committee:

James C. McDaniel, Advisor

Christopher P. Goyne, Chairman

Charles A. Sackett, Committee Member
Harsha K. Chelliah, Committee Member

Glenn S. Diskin, Committee Member

Accepted for the School of Engineering and Applied Science:

James H. Aylor, Dean

School of Engineering and Applied Science

August 2014 


\begin{abstract}
Scramjets are an appealing alternative to traditional rocket engines for many applications such as access to space, long-range strike missile capabilities, and civilian transport. Their advantages over rockets and traditional jet engines include a stationary flow path with no moving parts and higher payload capacity due to their airbreathing operation. A major hindrance to the maturation of scramjet technology is the high-cost and high-risk associated with flight testing. Computational fluid dynamics (CFD) simulations are a promising alternative to flight testing, but much of the complex flow physics present in scramjet engines are not well understood, making the modeling of these high-enthalpy, supersonic, combusting, and three-dimensional flows extremely challenging. Ground testing of scramjet model flow paths allows for experimental measurements of key variables, such as temperature and species number density, in controlled experimental environments. These measurements are guided by preliminary CFD results while also serving to validate the advanced state-of-the-art computational models. This symbiotic relationship between ground test experiments and computational models brings us closer to the realization of operational scramjet technology.

Harsh flowfields, such as those found within a scramjet, can present great difficulties for conventional in-stream diagnostics. The high speed, high enthalpy, combusting flowfields make probing the flow very challenging. Optical diagnostics, such as tunable diode laser (TDL) techniques, have an advantage in these harsh flowfields over their mechanical counterparts due to their non-intrusive nature. No hardware comes in contact with the flow itself, and therefore the integrity of the diagnostic is preserved and the flow is undisturbed. Traditional implementations of TDL, such as Tunable Diode Laser Absorption Spectroscopy (TDLAS), are indeed non-intrusive, but are limited by their path-integrated line-of-sight (LOS) nature. The harsh flowfields of a scramjet are highly three-dimensional and thus an optical diagnostic technique capable of producing spatially resolved measurements is required. Tunable Diode Laser Absorption Tomography (TDLAT) is a non-intrusive optical technique which combines TDLAS and computed tomography (CT) to produce a 2-D spatially resolved measurement of two key combustion diagnostic properties: temperature and species number density. Several hundred LOS TDLAS measurements are collected for each experiment and are subsequently reconstructed utilizing a tomographic post-processing algorithm. The TDLAT measurement results in highly resolved 2-D temperature and species number density distributions of the flowfield at the probed measurement plane.

The work presented herein describes the development of the TDLAT technique, building upon earlier proof-of-concept demonstrations. Application of the TDLAT diagnostic to two scramjet-relevant ground test facilities is presented and the insight gained from the resulting 2-D distributions is discussed. Of great significance, the combustion efficiency of the University of Virginia's dual-mode scramjet is experimentally measured in situ, becoming the first measurement of its kind on a dual-mode
\end{abstract}


scramjet. The TDLAT technique is shown to be a novel and viable diagnostic technique which is capable of providing significant insight into the complex flowfields within scramjet engines. 


\section{Acknowledgements}

Dr. James McDaniel -

For believing in me and welcoming me to your University. Your quiet, but steadfast, support has allowed me to develop into the researcher I am today. You allowed me to ask

my own questions and pursue my own curiosities and for that I am very grateful.

Dr. Christopher Goyne -

For teaching me about the complexities of scramjet testing and guiding my research.

Dr. Bob Rockwell, Roger Reynolds, and Dr. Roland Krauss -

For the endless hours you spent helping me to design, fabricate, and build my experimental hardware, as well as for running the UVaSCF for my experiments. Your good-humored attitudes and genuine desire to help me succeed made the long test days endurable.

Dr. Glenn Diskin and Dr. Michael Brown -

For your unwavering guidance and support, both with technical and non-technical matters. Glenn, you first introduced me to the world of spectroscopy. You have always been willing and able to answer my unending questions, with an optimism and curiosity that I strive to match. Mike, you gave me the final push of support I needed to finish my Ph.D. You've taught me how important it is to be passionate about your work and to keep the "fire in your belly".

Dr. Harsha Chelliah and Dr. Cass Sackett -

For your guidance and for serving on my committee.

Brian Rice, Ben Tatman, Jesse Quinlan, Max DePiro, Thomas Steva, Justin Kirik, Mike Smayda, Josh Codoni, Brendyn Sarnacki, Christina Pappas, and Elliott

Bryner -

For being by my side every step of the way.

My family and friends back home -

For your encouragement and unconditional love. Thank you for believing in me when I had trouble believing in myself.

NASA, AFOSR, the National Science Foundation, and the Virginia Space Grant Consortium -

For their financial support. 


\section{Contents}

Acknowledgements $\quad$ i

Nomenclature $\quad$ xii

1 Introduction $\quad 1$

1.1 Dissertation Overview . . . . . . . . . . . . . . . . 1

1.2 Background and Motivation . . . . . . . . . . . . . . . 2

1.3 Research Objectives . . . . . . . . . . . . . . . . . . . . 10

2 Conceptual Model and Experimental Overview 12

2.1 Introduction . . . . . . . . . . . . . . . . . . . 12

2.2 Theory . . . . . . . . . . . . . . . . . . . . 12

2.2.1 TDLAS Theory . . . . . . . . . . . . . . . . . . 13

2.2.2 Computed Tomography Theory . . . . . . . . . . . . . . . 17

2.3 Experimental Overview . . . . . . . . . . . . . . . . . . 20

2.3.1 Hardware Design . . . . . . . . . . . . . . . . . . 20

2.3.2 Optical Design . . . . . . . . . . . . . . . . . . 22

2.4 Three-phase Analysis . . . . . . . . . . . . . . . . . . . . . . 32

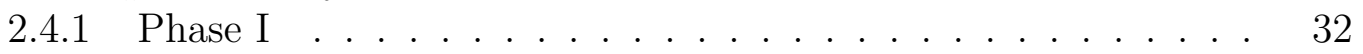

2.4 .2 Phase II . . . . . . . . . . . . . . . . . . 46

2.4 .3 Phase III . . . . . . . . . . . . . . . . . . . . . . . . . . . . . 47

3 Tomographic Processing and Error Evaluation $\quad 49$

3.1 Introduction and Motivation . . . . . . . . . . . . . . . . . . 49

3.2 Method ........................ 56

3.3 Impact of Background Subtraction . . . . . . . . . . . . . 67

3.4 Uncertainty Analysis for TDLAT Measurements . . . . . . . . . . . . 75

4 UVa Results $\quad 78$

4.1 Introduction . . . . . . . . . . . . . . . . . 78

4.2 Experimental Overview . . . . . . . . . . . . . . . . . . . . . . . . . . . . . . . 79

4.2 .1 Facility . . . . . . . . . . . . . . . . . 79

4.2.2 Experimental Setup . . . . . . . . . . . . . . 83

4.2.3 Calibration of Physical Orientation . . . . . . . . . . . . 86

4.3 Case 1: $12 \%$ Steam Results . . . . . . . . . . . . . . . . 90 
4.4 Case 2: Scram-mode $\phi=0.18$ Results . . . . . . . . . . . . . 103

4.5 Case 3: Ram-mode $\phi=0.49$ Results . . . . . . . . . . . . . . . 116

4.6 Combustion Efficiency . . . . . . . . . . . . . . . . . 127

5 NASA Langley Results 133

5.1 Introduction and Motivation . . . . . . . . . . . . . . 133

5.2 NASA Langley Direct-Connect Supersonic

Combustion Test Facility . . . . . . . . . . . . . . . . . . . . . . . 134

5.3 Experimental Parameters . . . . . . . . . . . . . . . . . . . . 139

5.4 Case 4: NASA Langley DCSCTF Results . . . . . . . . . . . . . . . . 140

6 Summary and Future Work 153

6.1 Summary . . . . . . . . . . . . . . . . . . . 153

6.1.1 TDLAT Hardware and Software Summary . . . . . . . . . . . 154

6.1.2 UVa Measurements Summary . . . . . . . . . . . . . . 155

6.1.3 NASA Langley Measurements Summary . . . . . . . . . . . . 157

6.2 Future Work . . . . . . . . . . . . . . . . . . . 158 


\section{List of Figures}

1.1 "Over and under" TBCC concept. [1] . . . . . . . . . . . . . .

1.2 X-51A flight-test scramjet attached to a B-52 before separating and igniting, reaching Mach 5+ under powered flight for approximately 300 seconds. [6] . . . . . . . . . . . . . . . .

1.3 Portrayal of optical techniques stemming from tunable diode laser direct absorption spectroscopy. . . . . . . . . . . . . .

2.1 Example of Boltzmann plot analysis with three representative absorption features. . . . . . . . . . . . . . . . . . .

2.2 Algebraic Reconstruction Technique: finite series expansion of discretized measurement. [31] . . . . . . . . . . . . . . . . . . . . 18

2.3 (a) Fanbeam style geometry, and (b) parallel beam style geometry. . . 21

2.4 Orientation of fanbeam collection geometry in both (a) measurement space, and (b) sinogram space. . . . . . . . . . . . . . .

2.5 (a) Temperature-dependent line strength of the four selected absorption features, and (b) ratio of temperature-dependent line strengths for various line pairs. . . . . . . . . . . . . . . .

2.6 Illustration of three temporally multiplexed lasers. Not to scale. . . .

2.7 First iteration of the optical TED box for TDLAT experiment, designed by Bryner and Snyder. [31] . . . . . . . . . . . . . . . .

2.8 TED box 2.0 for use in measurements on NASA Langley DCSCTF (a) in dimensioned SolidWorks model, (b) SolidWorks isometric view, and (c) fabricated and coated in absorptive Aeroglaze paint. Solid models of Thorlabs components property of Thorlabs, Inc. . . . . . . . . . .

2.9 TED box 3.0 for use in measurements on UVaSCF (a) in dimensioned SolidWorks model, (b) SolidWorks isometric view, and (c) mounted at the exit plane of the UVaSCF. Solid models of Thorlabs components property of Thorlabs, Inc. . . . . . . . . . . . . . . . .

2.10 (a) Bandpass filter utilized in TED 2.0 and 3.0, and (b) aspheric lens utilized in TED 2.0 and 3.0. . . . . . . . . . . . . . . . . . 32

2.11 Example plot of raw measured signals: current, signal, and etalon data. 33

2.12 (a) An example of single-scan LOS signal and the identified average background level between laser-on periods, and (b) the same singlescan LOS signal with the linear background removed. . . . . . . . . 
2.13 (a) An example of calculating the mean signal from 150 single-scan LOS, and (b) the mean etalon from 150 single-scan LOS, and (c) the mean current from 150 single-scan LOS. . . . . . . . . . . . . . .

2.14 Representative signals for all three lasers after averaging over 150 single-scans. . . . . . . . . . . . . . . . . . . 35

2.15 Optical setup for etalon detection and frequency retrieval. . . . . . . 36

2.16 (a) An example of an etalon trace with the extrema identified, and (b) the resulting point number to relative frequency conversion. . . . . . 37

2.15 Preliminary baseline fit for (a) KB1, (b) KB2, (c) KB3, and (d) KB4. 40

2.16 Diagram of Whiting approximation of Voigt profile. [52] . . . . . . . 41

2.17 Sample multi-peak and single-peak Voigt fits for (a) KB1, (b) KB2, (c) KB3, and (d) KB4. . . . . . . . . . . . . . . . . . .

2.18 Convergence of ML-EM algorithm for sample pixels. (a) Pixel value versus iteration number for three sample pixels, and (b) the derivative of the pixel values with respect to the iteration number. . . . . . . .

3.1 Schematic of region of interest. . . . . . . . . . . . . . 50

3.2 (a) Unaltered KB1 sinogram, and (b) unaltered KB1 reconstruction. . $\quad 52$

3.3 (a) Distribution of total laser path length per pixel, $\sum_{i} A_{i j}$, for experimental parameters shown in Table 2.1, and (b) normalized radial gradient of total laser path length per pixel, with a single fanbeam superimposed in white. . . . . . . . . . . . . . .

3.4 (a) Zero-background original distribution, (b) zero-background reconstruction, (c) zero-background error, (d) zero-background normalized sinogram, (e) non-zero background original distribution, (f) non-zero background reconstruction, (g) non-zero background error, and (h) non-zero background normalized sinogram. . . . . . . . . . . .

3.5 Synthetically created distributions with $0 \%$ noise added. Properties of Figures 3.5a-3.5i are summarized in Table 3.1. The distributions are: (a) CFD absorbance, where the colorbar is scaled by $\times 10^{-5}$, and synthetically generated (b) through (i), which are distributions $2-9$. .

3.6 Synthetically created distributions with 30\% noise added. Properties of Figures 3.6a-3.6i are summarized in Table 3.1. The distributions are: (a) CFD absorbance, where the colorbar is scaled by $\times 10^{-5}$, and synthetically generated (b) through (i), which are distributions 2-9. .

3.7 (a) The evolution of a first example point for varied levels of background subtraction across varying grid radius, and (b) the evolution of a second example point for varied levels of background subtraction across varying grid radius. . . . . . . . . . . . . . .

3.8 The standard deviation for the first sixty points of distribution 2 with $0 \%$ noise addition for all seven background subtraction values. . . . .

3.9 (a) The mean of pixel-by-pixel variations for distribution 2 for all four noise addition cases versus the \% subtraction case, and (b) the standard deviation of pixel-by-pixel variations for distribution 2 for all four noise addition cases versus the $\%$ subtraction case. . . . . . . . . . . 
3.10 The mean of pixel-by-pixel variation versus subtraction case for all noise addition cases for distributions 1-9. . . . . . . . . . . . .

3.11 Summary of identification of correct background value for nine synthetic distributions. . . . . . . . . . . . . . . . .

3.12 Mean relative error plots for the baseline, $100 \%$ subtraction, and $110 \%$ subtraction cases for all nine synthetic distributions as a function of reconstruction grid radius. . . . . . . . . . . . . . . . . .

3.13 (a) Mean relative error at $50 \mathrm{~mm}$ reconstruction grid radius for baseline, $100 \%$ subtraction, and $110 \%$ subtraction cases, and (b) \% improvement over baseline case for $100 \%$ subtraction and $110 \%$ subtraction. 70

3.14 Mean pixel-by-pixel variance for background substitution attempt for (a) case 1 experimental data, and (b) case 2 experimental data. . . .

3.15 Black pixels represent LOS which have little interaction with Region A and white pixels represent LOS which intersect Region A. . . . . . 72

3.16 Edge rays divided by path length for synthetic distribution 2 , for four cases of noise addition. . . . . . . . . . . . . . . .

3.17 (a) Background subtraction values for the synthetic distributions identified via the simplified background subtraction method, and (b) relative error resulting from the worst-case guess of the background subtraction values. . . . . . . . . . . . . . . . . . .

3.18 Resulting background absorbance values for each absorption feature and each experimental data set using simplified background subtraction method. . . . . . . . . . . . . . . . . . .

4.1 UVaSCF (a) schematic, and (b) test section hardware for Configuration C. . . . . . . . . . . . . . . . . . . .

4.2 UVaSCF Configuration $\mathrm{C}$ flow path and characteristic pressure scans for three operating points. . . . . . . . . . . . . . . .

4.3 Schematic of (a) single-box configuration for TDLAT measurements on UVaSCF facility, and (b) multi-box configuration for TDLAT measurements on NASA Langley DCSCTF facility. . . . . . . . . . . . . .

4.4 TDLAT hardware mounted on UVaSCF (a) full view, and (b) close up view at measurement plane. . . . . . . . . . . . . . .

4.5 Mapping from reconstruction grid space to physical space with solid objects both inside and outside the tunnel flow path hardware. The first column shows (a) a photograph, (c) reconstruction, and (e) sinogram of the mapping of objects placed within the flow path. The second column shows (b) a photograph, (d) reconstruction, and (f) sinogram of the mapping of objects placed outside of the flow path. . . . . . . .

4.6 Orientation of the presented data to the actual UVaSCF. View is looking from above, with flow coming out of the page. The fuel injector is on the lower horizontal wall. (a) The view in which data will be presented, and (b) SolidWorks model of UVaSCF containing entire flow

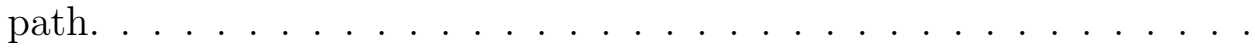


4.7 Integrated absorbance sinograms at the exit plane of the UVaSCF for case 1 . The first column contains the raw sinograms of TDLAT measured integrated absorbance for each of the four absorption features (KB1-KB4) and the second column contains the corresponding filtered sinograms. . . . . . . . . . . . . . . . . . 91

4.8 LOS temperatures calculated for case 1 TDLAT measurements. . . . 93

4.9 Reconstructed integrated absorbance at the exit plane of the UVaSCF for case 1, for (a) KB1, (b) KB2, (c) KB3, and (d) KB4. . . . . . . . 94

4.10 Two-line thermometry calculation via Equation 2.10 at the exit plane of the UVaSCF for case 1, for line-pairs (a) KB1 and KB2, (b) KB1 and KB3, (c) KB1 and KB4, (d) KB2 and KB3, and (e) KB2 and KB4. 95

4.11 Root-mean square error for Boltzmann plot at the exit plane of the UVaSCF for case 1. . . . . . . . . . . . . . . . . . . 96

4.12 Final reconstructions at the exit plane of the UVaSCF for case 1: (a) static temperature, (b) water vapor number density, and (c) water vapor mole fraction. . . . . . . . . . . . . . . . . . . .

4.13 Coherent anti-Stokes Raman Spectroscopy (CARS) measurement of rotational temperature at the in-flow plane of the combustor, $\mathrm{x} / \mathrm{H}=$

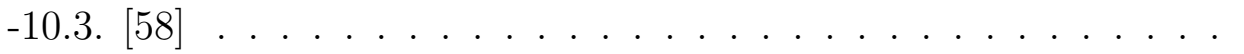

4.14 Case 1 line plots (profiles) both vertical and horizontal of (a) vertical temperature profile, (b) horizontal temperature profile, (c) vertical water vapor number density profile, (d) horizontal water vapor number density profile, (e) vertical water vapor mole fraction profile, and (f) horizontal water vapor mole fraction profile. . . . . . . . . . . .

4.15 Path-integrated TDLAT measurement compared to 1-D Stanford Measurement at the exit plane of the UVaSCF for case 1. . . . . . . . . 100

4.16420 stationary LOS collected at case 1 conditions. . . . . . . . . . . . 101

4.17 Absolute uncertainty and \% of full value uncertainty for case 1 reconstructions of temperature, water vapor number density, and water vapor mole fraction. . . . . . . . . . . . . . . . . . .

4.18 Integrated absorbance sinograms at the exit plane of the UVaSCF for case 2 . The first column contains the raw sinograms of TDLAT measured integrated absorbance for each of the four absorption features (KB1-KB4) and the second column contains the corresponding filtered sinograms. . . . . . . . . . . . . . . . . . . . 104

4.19 LOS temperatures calculated for case 2 TDLAT measurements. . . . 105

4.20 Reconstructed integrated absorbance at the exit plane of the UVaSCF for case 2, for (a) KB1, (b) KB2, (c) KB3, and (d) KB4. . . . . . . . 106

4.21 Two-line thermometry calculation via Equation 2.10 at the exit plane of the UVaSCF for case 2 for line-pairs (a) KB1 and KB2, (b) KB1 and KB3, and (c) KB2 and KB3. . . . . . . . . . . . . . . . . . 107

4.22 Root-mean square error for Boltzmann plot at the exit plane of the UVaSCF for case 2. . . . . . . . . . . . . . . . . . . . 107 
4.23 Final reconstructions at the exit plane of the UVaSCF for case 2: (a) static temperature, (b) water vapor number density, and (c) water vapor mole fraction. . . . . . . . . . . . . . . . . . . . . . . . . . 108

4.24 Side-by-side comparisons of TDLAT measurements and CFD at the exit plane of the UVaSCF for case 2, (a) TDLAT temperature distribution, (b) CFD temperature distribution, (c) TDLAT water vapor number density distribution, (d) CFD water vapor number density distribution, (e) TDLAT water vapor mole fraction distribution, and (f) CFD water vapor mole fraction distribution. . . . . . . . . . . . .

4.25 Line plot comparisons of TDLAT measurements and CFD at the exit plane of the UVaSCF for case 2, (a) vertical profiles for TDLAT and CFD temperatures, (b) horizontal profiles for TDLAT and CFD temperatures, (c) vertical profiles for TDLAT and CFD water vapor number densities, (d) horizontal profiles for TDLAT and CFD water vapor number densities, (e) vertical profiles for TDLAT and CFD water vapor mole fractions, and (f) horizontal profiles for TDLAT and CFD water vapor mole fractions. . . . . . . . . . . . . . . . . . . . . 111

4.26560 stationary LOS collected at case 2 conditions. . . . . . . . . . . . 114

4.27 Absolute uncertainty and \% of full value uncertainty for case 2 reconstructions of temperature, water vapor number density, and water vapor mole fraction. . . . . . . . . . . . . . . . . . . .

4.28 Integrated absorbance sinograms at the exit plane of the UVaSCF for case 3. The first column contains the raw sinograms of TDLAT measured integrated absorbance for each of the four absorption features (KB1-KB4) and the second column contains the corresponding filtered sinograms. . . . . . . . . . . . . . . . . . . . 117

4.29 LOS temperatures calculated for case 3 TDLAT measurements. . . . 118

4.30 Reconstructed integrated absorbance at the exit plane of the UVaSCF for case 3, for (a) KB1, (b) KB2, (c) KB3, and (d) KB4. . . . . . . . 118

4.31 Two-line thermometry calculation via Equation 2.10 at the exit plane of the UVaSCF for case 3, for line-pairs (a) KB1 and KB2, (b) KB1 and KB3, and (c) KB2 and KB3. . . . . . . . . . . . . . . .

4.32 Root-mean square error for Boltzmann plot at the exit plane of the UVaSCF for case 3. . . . . . . . . . . . . . . . . . . . . . 120

4.33 Final reconstructions at the exit plane of the UVaSCF for case 3: (a) temperature, (b) water vapor number density, and (c) water vapor mole fraction. . . . . . . . . . . . . . . . . . . .

4.34 (a) Z-component of velocity, $V_{z}$, measured by Rice [60] at the exit plane of the UVaSCF for case 3, and (b) contour plot of water vapor mole fraction via TDLAT with overlaid in-plane velocity vectors via SPIV [60] at the exit plane of the UVaSCF for case 3. . . . . . . . . . . . 123 
4.35 Case 3 line plots (profiles) both vertical and horizontal of (a) vertical temperature profile, (b) horizontal temperature profile, (c) vertical water vapor number density profile, (d) horizontal water vapor number density profile, (e) vertical water vapor mole fraction profile, and (f) horizontal water vapor mole fraction profile. . . . . . . . . . .

4.36 Absolute uncertainty and \% of full value uncertainty for case 3 reconstructions of temperature, water vapor number density, and water vapor mole fraction. . . . . . . . . . . . . . . . . . .

4.37 Water vapor number density, axial velocity, and water vapor flux for scram-mode operation from TDLAT (first column) and CFD [55] (second column).

4.38 Water vapor number density from TDLAT measurement, axial velocity from SPIV measurement [60], and water vapor flux for the scram-mode (first column) and ram-mode (second column). . . . . . . . . . .

5.1 NASA Langley Direct-Connect Supersonic Combustion Test Facility with TDLAT system installed. . . . . . . . . . . . . . . . . . 135

5.2 Schematic of DCSCTF and location of TDLAT equipment. . . . . . . 135

5.3 TDLAT system used for measurements on (a) the UVaSCF, and (b) the NASA Langley DCSCTF. . . . . . . . . . . . . . . . . . . 136

5.4 SolidWorks model of the multi-box TDLAT configuration for use on the DCSCTF. . . . . . . . . . . . . . . . . . 137

5.5 Single-scan and averaged LOS sample data from DCSCTF TDLAT. . 138

5.6 Integrated absorbance sinograms at the exit plane of the DCSCTF for case 4. Sinograms are formed by combining the measurements collected during eight tunnel runs. The first column contains the raw sinograms of TDLAT measured integrated absorbance for each of the four absorption features (KB1-KB4) and the second column contains the corresponding filtered sinograms. . . . . . . . . . . . . . . . 141

5.7 LOS temperatures calculated for case 4 TDLAT measurements. . . . 142

5.8 Reconstructed integrated absorbance at the exit plane of the NASA Langley DCSCTF for case 4, for (a) KB1, (b) KB2, (c) KB3, and (d) KB4. . . . . . . . . . . . . . . . . . . . .

5.9 Two-line thermometry calculation via Equation 2.10 at the exit plane of the NASA Langley DCSCTF for case 4, for line-pairs (a) KB1 and KB2, (b) KB1 and KB3, and (c) KB2 and KB3. . . . . . . . . . . . . 144

5.10 Root-mean square error for Boltzmann plot at the exit plane of the NASA Langley DCSCTF for case 4. . . . . . . . . . . . . . . .

5.11 Final reconstructions at the exit plane of the NASA Langley DCSCTF for case 4: (a) temperature, (b) water vapor number density, and (c) water vapor mole fraction. . . . . . . . . . . . . . . . . . . . 146 
5.12 Side-by-side comparisons of TDLAT measurements and CFD at the exit plane of the NASA Langley DCSCTF for case 4, (a) TDLAT temperature distribution, (b) CFD temperature distribution, (c) TDLAT water vapor number density distribution, (d) CFD water vapor number density distribution, (e) TDLAT water vapor mole fraction distribution, and (f) CFD water vapor mole fraction distribution. . . . . . . .

5.13 Line plot comparisons of TDLAT measurements and CFD at the exit plane of the NASA Langley DCSCTF for case 4, (a) vertical profiles for TDLAT and CFD temperatures, (b) horizontal profiles for TDLAT and CFD temperatures, (c) vertical profiles for TDLAT and CFD water vapor number densities, (d) horizontal profiles for TDLAT and CFD water vapor number densities, (e) vertical profiles for TDLAT and CFD water vapor mole fractions, and (f) horizontal profiles for TDLAT and CFD water vapor mole fractions. . . . . . . . . . . . . . . .

5.14 Off-center temperature line plot comparisons of TDLAT measurements and CFD at the exit plane of the NASA Langley DCSCTF for case 4. 151

5.15 Off-center water vapor number density line plot comparisons of TDLAT measurements and CFD at the exit plane of the NASA Langley DCSCTF for case 4. . . . . . . . . . . . . . . . . . . . 151

5.16 Off-center water vapor mole fraction line plot comparisons of TDLAT measurements and CFD at the exit plane of the NASA Langley DCSCTF for case $4 . \ldots \ldots \ldots \ldots$. . . . . . . . . . . . . 152 


\section{List of Tables}

2.1 Fanbeam geometry parameters for both test facilities utilized.

2.2 Transitions used in TDLAT system and their spectroscopic parameters. $[16][48] \ldots \ldots \ldots \ldots \ldots \ldots$

3.1 Summary of properties of the nine synthetic distributions created for study, as shown in Figures 3.5 and 3.6. . . . . . . . . . . . . . 60

3.2 Summary of randomly generated parameters used in Equations 3.3a and 3.3b to create six of the nine synthetic distributions, as shown in Figures 3.5d-3.5i and Figures 3.6d-3.6i. . . . . . . . . . . . . . .

3.3 Summary of mean relative error for the baseline, $100 \%$ subtraction, and $110 \%$ subtraction cases, at each level of noise addition. . . . . . .

3.4 Summary of background absorbance values for each absorption feature and each experimental data set. . . . . . . . . . . . . . . .

4.1 Summary of UVaSCF operating conditions for TDLAT measurement collection. . . . . . . . . . . . . . . 80

4.2 Statistical properties of case 1 measurements. . . . . . . . . . . . . . 101

4.3 Statistical properties of case 2 measurements. . . . . . . . . . . . . . 114

4.4 Summary of UVaSCF combustion efficiency via TDLAT \& SPIV. . . 130

5.1 Test conditions during TDLAT measurements on DCSCTF. . . . . . 140 


\section{Nomenclature}

$\alpha \quad$ Total angle inscribed by fanbeam, ${ }^{\circ}$

$\alpha_{D} \quad$ Doppler width, $\mathrm{cm}^{-1}$

$\alpha_{L} \quad$ Lorentz width, $\mathrm{cm}^{-1}$

$\Delta \alpha \quad$ Ray angle, ${ }^{\circ}$

$\Delta \nu_{f s r}$ Free spectral range of interference pattern, $\mathrm{Hz}$

$\Delta \theta \quad$ Fan angle, ${ }^{\circ}$

$\dot{N}_{\text {Hydrogen,injected }}$ Total mass flow rate of $H_{2}$ injected into the UVaSCF, grams/s

$\eta_{c} \quad$ Combustion efficiency

$\hat{P}_{i} \quad$ Measured LOS projection for the $i^{\text {th }}$ ray

$\kappa_{\nu} L \quad$ Absorbance, $\mathrm{cm}^{-1}$

$\lambda \quad$ Wavelength

$\lambda_{C L} \quad$ Wavelength at line center

$\nu, \nu_{0}$ frequency and transition line center frequency, $\mathrm{cm}^{-1}$

$\phi \quad$ Fuel-to-air equivalence ratio

$\phi_{\nu} \quad$ Lineshape function, $\mathrm{cm}$

$\tau_{m} \quad$ Abrarov constant

$\theta_{f_{i}} \quad$ Principal angle between fan $i$ and y-axis of Cartesian plane, ${ }^{\circ}$

$A_{i} \quad$ Spectrally integrated area of $i^{\text {th }}$ absorption feature, $\mathrm{cm}^{-1}$

$a_{n} \quad$ Abrarov series coefficient

$A_{i j} \quad$ Geometric area matrix

Amp Amplitude multiplier for Abrarov Voigt approximation 
$b(z)$ Baseline terms in Abrarov approximation

$B(x, y)$ Original value of background pixels in synthetic distribution

c $\quad$ Speed of light in a vacuum, $299,792,458 \mathrm{~m} / \mathrm{s}$

$c_{0} \quad$ DC coefficient of Boltzmann plot analysis

$c_{1} \quad$ Linear coefficient of Boltzmann plot analysis

$d_{f s r} \quad$ Path length of the interference cavity, $\mathrm{m}$

$D_{\text {new }}(x, y)$ Altered synthetic distribution

$D_{\text {original }}(x, y)$ Original synthetic distribution

E Sum of squared errors for Abrarov Voigt approximation fit to measured data

$E_{i}^{\prime \prime} \quad$ Lower-state energy level of the $i^{\text {th }}$ absorption feature, $\mathrm{cm}^{-1}$

$h \quad$ Planck's constant, $6.62606957 \times 10^{-34} \mathrm{~m}^{2} \mathrm{~kg} / \mathrm{s}$

I Transmitted intensity

$I_{0} \quad$ Incident intensity

$I_{\lambda} \quad$ Wavelength-dependent intensity

$I_{\lambda_{C L}} \quad$ Wavelength-dependent intensity at line center

$K \quad$ Voigt function

$K(x, y)$ Real component of complex error function

$k_{B} \quad$ Boltzmann's constant, $1.3806488 \times 10^{-23} \mathrm{~m}^{2} \mathrm{~kg} \mathrm{~s}^{-2} \mathrm{~K}^{-1}$

L Path length, cm

$L(x, y)$ Imaginary component of complex error function

$N \quad$ Number of terms in Abrarov complex error function approximation sum, equal to 32 for this research

$n \quad$ Index of refraction of medium through which light travels

$N_{A} \quad$ Avagadro's number, $6.02214 \times 10^{23}$ molecules $/$ mole

$N_{\text {fan }}$ Total number of fans per data set

$N_{\mathrm{H}_{2} \mathrm{O}}(y, z)$ Spatially resolved water number density distribution, molecules $/ \mathrm{cm}^{3}$

$N_{\text {ray }}$ Total number of rays per fanbeam 
$N_{\text {total }}$ Total number density of a gas mixture

O Sample distribution

$P \quad$ Static pressure, atm

$p \quad$ Intensity constant for synthetic distribution noise addition

$p_{i} \quad i^{t h}$ Re-projection

$q \quad$ Iteration number

$Q(T)$ Partition function of the absorbing feature at temperature $T$

$r(x, y)$ Matrix of pseudo-random numbers from a standard normal distribution

$R \quad$ Reconstruction ratio, number of measurements divided by number of reconstruction grid cells

$R \quad$ Universal gas constant, $82.05736 \mathrm{~cm}^{3}$ atm $\mathrm{K}^{-1} \mathrm{~mol}^{-1}$

$r \quad$ Radius between $s_{i}$ and origin of Cartesian plane, $\mathrm{mm}$

$S(T)$ Line strength, $\mathrm{cm}^{-1} /$ molecule- $\mathrm{cm}^{-2}$

$s_{i} \quad i^{t h}$ fan vertex

$T \quad$ Static temperature, $K$

$T_{\nu} \quad$ Transmittance

$T_{\text {ref }} \quad$ HITRAN Reference temperature, $296 K$

$V(z)$ Abrarov Voigt approximation

$V_{x}(y, z)$ Spatially resolved axial velocity distribution, $\mathrm{m} / \mathrm{s}$

$w(z)$ Abrarov complex error function approximation

$W(z)$ Complex error function

$w_{L} \quad$ Lorentzian full width at half-maximum

$w_{V} \quad$ Voigt full width at half-maximum

$x, y, z$ Input parameters to Abrarov Voigt approximation

$x_{j} \quad$ Reconstruction variable at the $j^{\text {th }}$ cell

$x_{\text {Boltzmann }}$ Abscissa-coordinate utilized in Boltzmann plot analysis, equal to the lowerstate energy of the corresponding absorption feature

$X_{\mathrm{H}_{2} \mathrm{O}}$ Water vapor mole fraction 
$y_{\text {Boltzmann }}$ Log-scale ordinate-coordinate utilized in Boltzmann plot analysis. Dependent on integrated absorbance, line strength at reference temperature, and lower-state energy 


\section{Chapter 1}

\section{Introduction}

\subsection{Dissertation Overview}

This dissertation is organized into six chapters. The first of the chapters is intended to provide the reader with the motivation for the research discussed herein, as well as contextualize this research historically. The research objectives of this body of work are explicitly presented. Chapter 2 introduces the reader to the concepts which will be utilized throughout the dissertation including: theory, an overview of the facilities utilized for experimental measurements, and a discussion of the threephase analysis procedure. Chapter 3 presents a more in-depth examination of the tomographic algorithm utilized, including analysis and mitigation of reconstruction artifacts. Uncertainty analysis of the measurement technique is also discussed. Chapter 4 presents experimental results for testing conducted at the University of Virginia, while Chapter 5 shows experimental results for testing conducted at NASA Langley Research Center, conveying the evolution and adaptability of the technique. Chapter 6 summarizes the findings of this body of work and it is verified that the proposed research objectives have been addressed. The original contributions of the author and greater impact of this work is established. 


\subsection{Background and Motivation}

Airbreathing propulsion systems are an appealing alternative to traditional rockets for hypersonic flight vehicle applications such as access to orbit. An airbreathing engine does not need to carry its oxidizer on board, and thus is able to carry a heavier payload. However, the hypersonic airbreather is more complex than a conventional rocket due to the external physics of high-speed aerothermodynamics and the internal propulsion flow path physics. One such high-speed airbreathing propulsion system is the (sc)ramjet engine.

A ramjet is a high-speed airbreathing propulsion device that exploits aerodynamic compression due to isentropic shocks off of the vehicle forebody (rather than turbomachinery) for the initial pressure-rise in preparation for fuel injection and combustion. Though a ramjet produces no static-thrust, at conditions near Mach 3 they become practical. The incoming freestream air is decelerated through a series of oblique shock waves, and finally a normal shock, which decelerates the flow to subsonic speeds in a 1-D sense. The subsonic air enters a combustor section where fuel is injected, ignited, and combusted. The resulting hot gases are then accelerated through a convergingdiverging nozzle to produce thrust.

A supersonic combustion ramjet, or scramjet, is similar to a ramjet, but operates in Mach number regimes of approximately 6-10. The high-enthalpy freestream air enters the scramjet engine through a series of oblique shock waves off of the vehicle forebody, but unlike in the ramjet mode, the flow remains supersonic in a 1-D sense throughout the internal flow path of the scramjet, creating an extremely challenging environment for the combustion process to fully mature. The supersonic air enters the combustor section, where again fuel is injected, ignited, and combusted. Due to the high velocity of the flow, the combustion process must be achieved in a very short time frame. The hot combustion products are then accelerated to ambient atmospheric pressure through a divergent nozzle, producing a thrust force on the vehicle. The flowfields within and exiting from (sc)ramjets are harsh, complex, and 
highly three-dimensional and are not fully understood.

(Sc)ramjets are unable to produce static thrust and thus cannot operate from takeoff to high-speed cruise velocities. Two combined cycle propulsion systems which are capable of spanning this operational range have been studied: the rocket-based combined cycle (RBCC) and the turbine-based combined cycle (TBCC). The fundamental flow physics between the RBCC and TBCC are essentially the same, so for brevity, the Turbine-Based Combined Cycle (TBCC) will be discussed herein.

Current TBCC conceptual design is known as an "over-and-under" TBCC engine, and is shown in Figure 1.1. The low-speed turbine flow path is situated parallel to and directly above the high-speed (sc)ramjet flow path. The engine transitions from the low-speed turbine flow path to the high-speed flow path at velocities nearing Mach 3-4 by closing the low-speed inlet and opening the high-speed inlet. This process is the first critical combined cycle mode transition. As the Mach number is increased past $\sim 4$, the subsonic ramjet transitions into the dual-mode regime. The dual-mode arises when the combustor inlet Mach number is high enough to alleviate the thermal throat in the combustor and the pre-combustion shock train in the isolator terminates with an oblique shock rather than a normal shock. The isolator flowfield contains multiple shock reflections, creating complex shock-boundary-layer-interactions (SBLI). As the Mach number is increased beyond $\sim 6$, the pre-combustion shock train moves out of the isolator and the combustor operates in a purely supersonic mode.

Historically, flight testing of (sc)ramjet engines has been erratic, enduring both triumphant victories and disconcerting breakdowns. National interest and hence funding has also seen peaks and valleys. One of the earliest attempts to flight-test a ramjet engine was during the X-15 program. Richard Weber developed the first definitive analytical evaluation of a supersonic ramjet in 1954, and the first open forum on the possibility of hypersonic flight was held at the 4th Advisory Group for Aerospace Research \& Development Colloquium in Milan in April 1960. [2] The X-15A-2 flew several flights with a dummy scramjet attached to the modified airframe, though the 


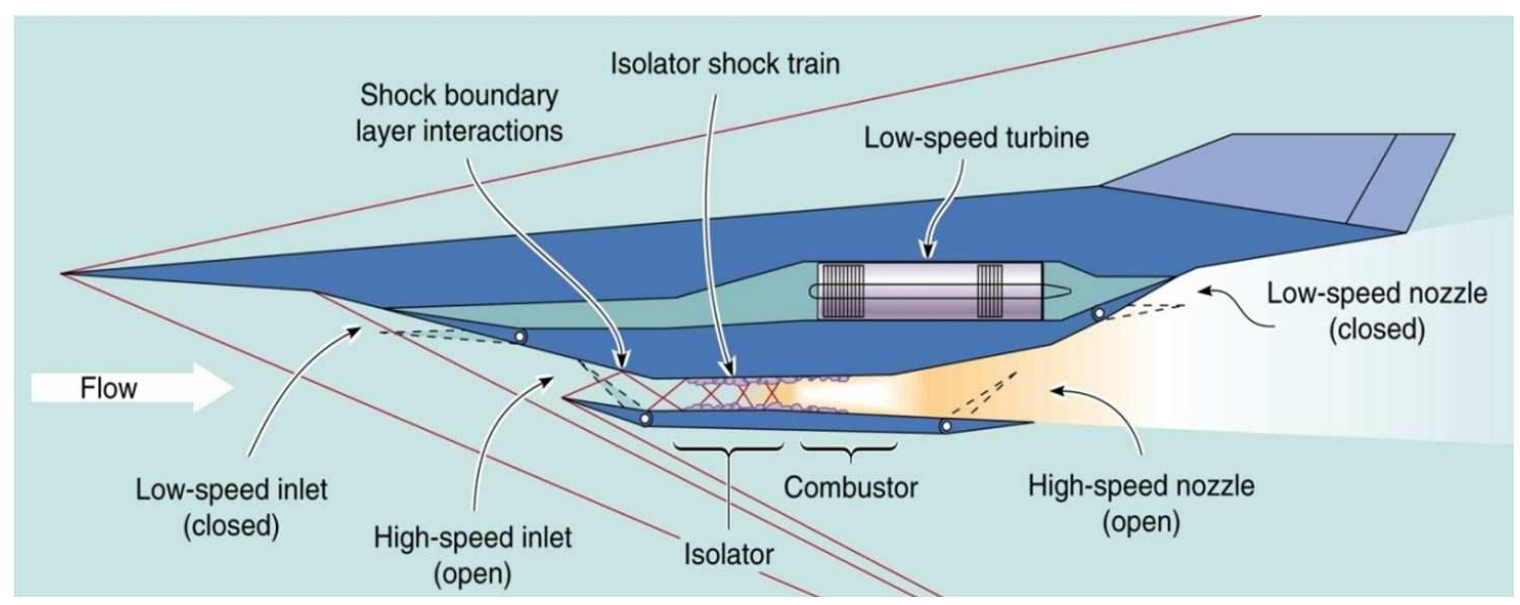

FiguRE 1.1: "Over and under" TBCC concept. [1]

possibility of flight tests with an actual scramjet were tempered when the scramjet pylon was severed by shockwaves and fell off of the plane. The National Aerospace Plane (NASP) program was made public in 1986, with plans for the development of two X-30 flight vehicles possibly reaching Mach 25. [3] The program was canceled in 1994 citing excessive costs (\$200-\$300 million annual budget from 1985 - 1993 [4]), becoming a significant and widely publicized disappointment for the US. Despite the ultimate termination of the project, the NASP program made many significant contributions to hypersonic flight technology, including Mach 18 testing of inlet, combustor, and small scale scramjets, Mach 8 testing of large scale ramjets, and Mach 16 testing of large scale scramjets. In addition to notable ground testing achievements, the NASP program led to substantial advancements in computational fluid dynamics (CFD) model development, validation, and analysis. [4] In 2002, Australia's HyShot program achieved the first flight test of a started scramjet engine with the flight of HyShot 2. [5] The HyShot 2 vehicle achieved supersonic combustion above Mach 7.5 via ballistic reentry into the earth's atmosphere. Most recently, the $\$ 300$ million $\mathrm{X}-51 \mathrm{~A}$ test program demonstrated air-breathing hypersonic flight above Mach 5 on May 1, 2013. [6][7][8][9] A photograph of the X-51A attached to a missile booster, carried by a B-52 is shown in Figure 1.2.

Due to the high-cost and high-risk nature of flight testing, other means of re- 


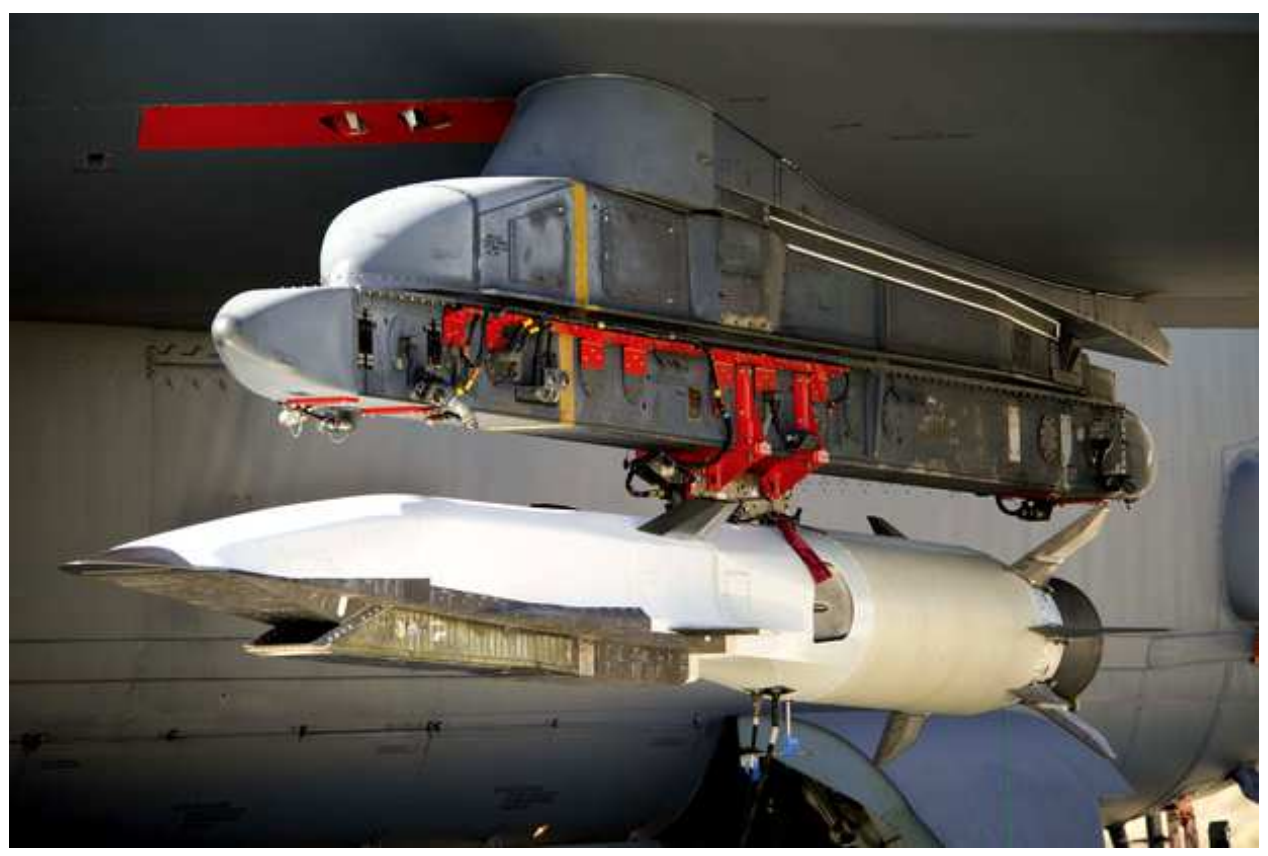

Figure 1.2: X-51A flight-test scramjet attached to a B-52 before separating and igniting, reaching Mach 5+ under powered flight for approximately 300 seconds. [6]

search will need to be employed to advance scramjet technology in the future. The $\$ 10$ million National Center for Hypersonic Combined Cycle Propulsion (NCHCCP), led by the University of Virginia, is facilitating a dual effort to develop advanced CFD models and generate one of the most complete experimental databases of a dual-mode scramjet facility to date. The complex nature of dual-mode, mixed subsonic/supersonic reacting flows demands the availability of quantitative measurements both to improve the understanding of the flow physics and for accurate CFD modeling using both a Reynolds-averaged Navier-Stokes (RANS) scheme, and a hybrid scheme which incorporates both Large Eddy Simulation (LES) and RANS (LES/RANS). Experimentally measured parameters such as temperature, species concentration, and combustion efficiency serve to validate the advanced modeling techniques and improve the understanding of the underlying flow physics.

Flowfields have historically been measured using techniques such as gas sampling probes and static pressure measurements along wind tunnel walls. However, when working in the supersonic regime, flows are very sensitive to disturbances, and intro- 
ducing probes into the flow will alter the flow properties. Therefore, a non-intrusive measurement technique is required. Planar optical measurement techniques, such as Planar Laser-Induced Fluorescence, are suitable for the supersonic regime but usually not for quantitative measurements. Coherent anti-Stokes Raman spectroscopy (CARS) has the ability to quantitatively measure the temperature and species concentration with spatial resolution, but requires a large series of single-point measurements taken sequentially and interpolated between to produce a "planar" measurement. This involves a very complex experimental setup and spectra to be measured several thousand times. The Tunable Diode Laser Absorption Tomography (TDLAT) technique, to be developed and applied in this research, is capable of quantitative and spatially-resolved measurements in the complex exhaust flowfields of a dual-mode scramjet model, providing both temperature and species number density distributions to the modelers.

The use of diode lasers for measuring gas properties originated in the 1970's following the demonstration of direct current injection semiconductor lasers. [10] In 1977, one of the earliest demonstrations of using tunable IR diode lasers to non-intrusively acquire in situ measurement of properties of combusting gases was by Hanson et al. [11] The field of diode laser absorption sensing has grown rapidly since the maturation of the telecommunications industry yielded widely available light sources and fiber optics for the visible and near-infrared spectral regions. In the 1980's, both Eckbreth and Kimball-Linne et al. published work on the advancement of optical tunable diode laser measurements in combustion. [12][13] In 1987, Silver and Stanton demonstrated airborne measurements of humidity (water vapor) using a diode laser. [14]

Much of the progress in tunable diode laser sensing was facilitated by the highresolution transmission molecular absorption database (HITRAN), which was established by the Air Force Geophysics Laboratory in 1960 for a better understanding of the transmission parameters of the earth's atmosphere. In 1986, and again several times in the decades since, a more complete version of HITRAN was released 
by Rothman et al. [15] A similar database, HITEMP was published in 2010 by Rothman et al. [16] and served to provide more accurate transmission parameters at elevated temperatures, whereas HITRAN was developed more for atmospheric, lower-temperature applications. Several of the transmission parameters utilized in this research were obtained from HITEMP 2010.

As is depicted in Figure 1.3, many techniques have stemmed from the fundamental idea of tunable diode laser direct absorption spectroscopy, including the branches of multi-species and multi-wavelength applications [17][18][19][20], velocity and mass flux measurement [21][22][23], wavelength modulation spectroscopy (WMS) [24][25][26][27][28][29], and spatially resolved techniques [30][31][32]. Each branch of this field serves to improve the fundamental direct absorption technique in their own way: simultaneously measuring multiple species, extracting additional properties from the flow, increasing signal-to-noise ratio, and obtaining spatially-resolved "planar" information, respectively. The research reported herein aligns with the Tomographic Reconstruction branch, highlighted in red in Figure 1.3.

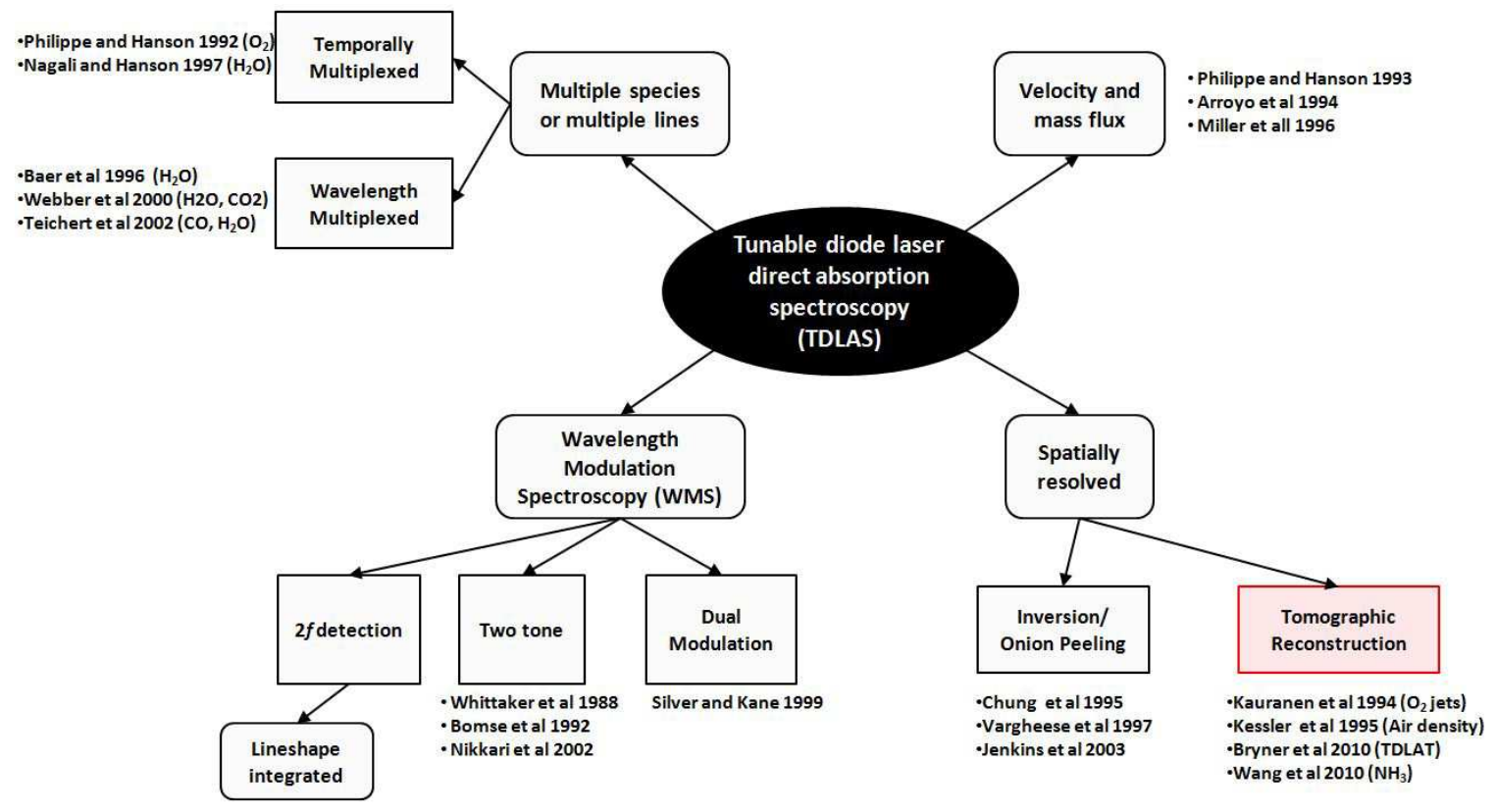

Figure 1.3: Portrayal of optical techniques stemming from tunable diode laser direct absorption spectroscopy. 
The development of Tunable Diode Laser Absorption Tomography (TDLAT) stems from the widely employed technique of Tunable Diode Laser Absorption Spectroscopy (TDLAS). TDLAS has been utilized for a range of applications, including sensing gaseous temperature, species concentration, velocity, and mass flux. TDLAS is excellent for use in probing high-speed, high-enthalpy, and combustion flowfields due to its robust and nonintrusive nature. TDLAS is intrinsically an integrated line-of-sight (LOS) measurement, and as such is limited by this 1-dimensional characteristic when large gradients of flow properties exist in the flowfield of interest. The TDLAT technique has been developed at the University of Virginia to augment traditional TDLAS approaches with a tomographic post-processing routine. This development has allowed for the favorable characteristics of TDLAS to be preserved, such as its nonintrusive nature, robust and commercially available optics, and ability to measure several flow properties rapidly, while enhancing its suitability to 3-dimensional flowfields.

The development of the TDLAT technique has many similarities to the work of Brown et al. and Nadir et al., who have also employed tomographic reconstruction techniques to spatially resolve absorption spectroscopy measurements in hypersonic flowfields. [33][34][35] Brown et al. have successfully measured the temperature and water vapor mole fraction distributions at the exit plane of the Hypersonic International Flight Research Experimentation (HIFiRE) Direct-Connect Rig ground test engine, as well as within the HIFiRE Flight 2 test article at the exit of the combustor and just upstream of the bifurcated exhaust nozzle. [34] While the methodology of Brown's work and the TDLAT research presented herein have many similarities which have proven to be synergistic, the ultimate objectives of each technique are distinct. Brown's work strives to make tomographic measurements at engine locations where optical access is very limited. Though the resulting spatial resolution of Brown's tomographic measurements is lower than is resolved by TDLAT, Brown's computations and reconstructions are achieved at quasi real-time processing rates. The TDLAT 
technique presented is intended to produce tomographic distributions with high spatial fidelity in situations where optical access is less restricted and real-time processing is not imperative.

Beyond just quantifying spatially resolved thermodynamic properties, application of the TDLAT technique to a supersonic combustor model with hydrogen injection also allows for improved understanding of the combustion efficiency. Historically, combustion efficiency measurements utilize the combination of a calorimeter and wall pressure taps but not with great accuracy. [36] To directly measure the combustion efficiency of the supersonic combustor model with $H_{2}$ fuel, the total mass flow rate of the produced $\mathrm{H}_{2} \mathrm{O}$ vapor (and thus converted $\mathrm{H}_{2}$ molecules) is compared to the total mass flow rate of the injected $H_{2}$ fuel. To quantify the total mass flow rate of the produced $\mathrm{H}_{2} \mathrm{O}$ vapor exiting the supersonic combustor model, two experimental optical diagnostics are utilized: TDLAT and stereoscopic particle image velocimetry (SPIV). SPIV is a planar optical measurement technique which utilizes injected seed particles to track the flow. CCD (charge-coupled device) cameras capture double frames of these particles which are illuminated by a double-pulsed laser sheet. The displacement of the particles is determined at interrogation sub-regions and is combined with the known time delay between laser pulses to determine the instantaneous velocity. Two cameras, viewing from different perspectives, allow for all three components of velocity to be measured. The TDLAT measurements result in spatially resolved water vapor number density distributions at the exit plane, while the SPIV technique results in a $2-\mathrm{D}$ distribution of the $3-\mathrm{D}$ velocity vector field at the exit plane. Pointby-point flux is calculated by multiplying these two quantities, water vapor number density and axial velocity, by their elemental area. The total mass flow rate of the produced $\mathrm{H}_{2} \mathrm{O}$ vapor exiting the supersonic combustor model is then calculated by summing the point-by-point flux across the exit plane of the model combustor. The combustion efficiency of the hydrogen-air combustor is then calculated as the ratio of the total mass flow rate of produced $\mathrm{H}_{2} \mathrm{O}$ to the known injected hydrogen mass flow 
rate of the facility. The injected hydrogen mass flow rate is measured by a mass flow sensor. This approach is described by the following equation:

$$
\eta_{c}=\frac{\int_{A_{\text {exit }}} N_{H_{2} O}(y, z) V_{x}(y, z) \mathrm{d} A_{\text {exit }}}{\dot{N}_{\text {Hydrogen }, \text { injected }}}
$$

where $N_{H_{2} O}(y, z)$ is the spatially resolved water number density distribution from the TDLAT measurement, $V_{x}(y, z)$ is the axial velocity distribution from the SPIV measurement, and $\dot{N}_{\text {Hydrogen,injected }}$ is the total mass flow rate of hydrogen fuel injected into the combustor.

\section{$1.3 \quad$ Research Objectives}

As discussed previously, a major hindrance to the advancement of scramjet technology is the high-cost and high-risk of flight testing. Computational efforts alleviate some of the cost and risk of flight testing, but are in great need of experimental data for development and validation. Therefore, there is a need for a measurement technique applicable in ground testing which can: efficiently and accurately measure two-dimensional temperature and species number density distribution in high-speed and high-enthalpy flow fields, efficiently and accurately measure combustion efficiency of a (sc)ramjet engine, and be utilized for both long and short duration ground testing with the aspiration of eventual flight testing.

To address the challenges outlined, the following research objectives are proposed:

I Develop efficient and accessible (well-documented) analysis codes for both spectral and tomographic reconstruction analysis

II Measure temperature and $\mathrm{H}_{2} \mathrm{O}$ number density distributions at the exit plane of the University of Virginia's Supersonic Combustion Facility (UVaSCF) at two operating points: scram-mode operation and ram-mode operation 
III Measure combustion efficiency of the UVaSCF at the exit plane, in conjunction with SPIV measurements

IV Modify and improve first iteration of TDLAT hardware and software for extension of the TDLAT technique to other experimental facilities (NASA Langley Direct-Connect Supersonic Combustion Test Facility) 


\section{Chapter 2}

\section{Conceptual Model and}

\section{Experimental Overview}

\section{$2.1 \quad$ Introduction}

This chapter presents the concepts which will be utilized throughout the remainder of the dissertation: fundamental theory, experimental facility overviews, hardware and optical architectures, and the analysis approach.

\subsection{Theory}

The TDLAT technique is comprised of absorption spectroscopy and computed tomography techniques. First, absorption spectroscopy theory is utilized to analyze and prepare the collected data for tomographic inversion. Second, computed tomography is used to reconstruct the two-dimensional absorbance fields. Lastly, absorption spectroscopy theory is again utilized to obtain the thermodynamic properties of interest at each spatial location. 


\subsubsection{TDLAS Theory}

The Beer-Lambert law expresses the relationship between the transmittance of light through a gas mixture and the resulting optical absorption of individual species. The Beer-Lambert law states that the transmittance, $T_{\nu}$, which is the ratio of the transmitted intensity, $I$, to the incident intensity, $I_{0}$, is proportional to the exponential of the product of path length, $L[\mathrm{~cm}]$, and $\kappa_{\nu}$, the spectral absorption coefficient $\left[\mathrm{cm}^{-1}\right]$ of the absorbing species:

$$
T_{\nu}=\frac{I}{I_{0}}=\exp \left(-\kappa_{\nu} L\right)
$$

where $\kappa_{\nu} L$ is termed the absorbance. The spectral absorption coefficient is given by Equation 2.2:

$$
\kappa_{\nu}=S(T) \cdot \phi_{\nu} \cdot N_{H_{2} O}
$$

where $S(T)$ is the transition line strength $\left[\mathrm{cm}^{-1} /\right.$ molecule- $\left.\mathrm{cm}^{-2}\right], N_{\mathrm{H}_{2} \mathrm{O}}$ is the number density of the absorbing species, in this case $\mathrm{H}_{2} \mathrm{O}$ vapor, [molecules $\cdot \mathrm{cm}^{-3}$ ], and $\phi_{\nu}$ is the lineshape function $[\mathrm{cm}]$ approximated by a Voigt function. The Voigt function is the spectral lineshape resulting from the convolution of independent Doppler and Lorentzian line broadening mechanisms:

$$
\phi_{\nu}=\frac{1}{\alpha_{D}} \sqrt{\frac{\ln 2}{\pi}} K\left(\frac{\left(\nu-\nu_{0}\right)}{\alpha_{D}} \sqrt{\ln 2}, \frac{\alpha_{L}}{\alpha_{D}} \sqrt{\ln 2}\right)
$$

where $\alpha_{D}$ is the Doppler width, $\alpha_{L}$ is the Lorentz width, $\nu_{0}$ is the transition line center wavenumber, and $K$ is the Voigt function. The temperature dependent line strength can be calculated using: 


$$
\begin{aligned}
S(T)=S\left(T_{r e f}\right) \frac{Q\left(T_{r e f}\right)}{Q(T)} \exp & {\left[-\frac{h c E^{\prime \prime}}{k_{B} T}\left(\frac{1}{T}-\frac{1}{T_{r e f}}\right)\right] \times } \\
& {\left[1-\exp \left(-\frac{h c \nu_{0}}{k_{B} T}\right)\right] \times\left[1-\exp \left(-\frac{h c \nu_{0}}{k_{B} T_{r e f}}\right)\right]^{-1} }
\end{aligned}
$$

where $h$ is Planck's constant, $c$ is the speed of light, $k_{B}$ is Boltzmann's constant, $E^{\prime \prime}$ is the lower state energy level $\left[\mathrm{cm}^{-1}\right], \nu_{0}$ is the line center wavenumber of the selected transition, $T_{\text {ref }}$ is the HITRAN reference temperature, $296 K$, and $Q(T)$ is the partition function of the absorbing species. By substituting Equation 2.2 into Equation 2.1, rearranging, and integrating over $d \nu$ from $-\infty$ to $+\infty$ the integrated area under a line is given by:

$$
A=S(T) \cdot N_{H_{2} O} \cdot L
$$

where $A$ is the spectrally integrated area $\left[\mathrm{cm}^{-1}\right]$. The measured absorption spectra are fit using a Voigt function and the integrated areas under the transitions are calculated. Equation 2.4 is then combined with Equation 2.5 to form Equation 2.6:

$$
\begin{aligned}
\ln \left(\frac{A}{S\left(T_{r e f}\right)} e^{-h c E^{\prime \prime} / k_{B} T_{r e f}}\right. & \left.\left(1-e^{-h c \nu_{0} / k_{B} T_{r e f}}\right)\right)= \\
& \frac{-h c E^{\prime \prime}}{k_{B} T}+\ln \left(\frac{Q\left(T_{r e f}\right)}{Q(T)} N_{H_{2} O} L\right)+\ln \left(1-e^{-h c \nu_{0} / k_{B} T}\right)
\end{aligned}
$$

For the wavelengths and temperatures considered in this research, $\exp \left(-h c \nu_{0} / K_{B} T\right) \approx$ 0, and thus these terms can be neglected, simplifying Equation 2.6 to:

$$
\ln \left(\frac{A}{S\left(T_{r e f}\right)} e^{-h c E^{\prime \prime} / K_{B} T_{r e f}}\right)=\frac{-h c E^{\prime \prime}}{k_{B} T}+\ln \left(\frac{Q\left(T_{r e f}\right)}{Q(T)} N_{H_{2} O} L\right)
$$


Equation 2.7 can be rewritten by making the following substitutions:

$$
\begin{aligned}
y_{\text {Boltzmann }} & =\ln \left(\frac{A}{S\left(T_{\text {ref }}\right)} e^{-h c E^{\prime \prime} / K_{B} T_{r e f}}\right) \\
x_{\text {Boltzmann }} & =E^{\prime \prime} \\
c_{1} & =\frac{-h c}{k_{B} T} \\
c_{0} & =\ln \left(\frac{Q\left(T_{r e f}\right)}{Q(T)} N_{H_{2} O} L\right)
\end{aligned}
$$

Applying these substitutions, the Boltzmann plot method is expressed by Equation 2.9:

$$
y_{\text {Boltzmann }}=c_{0}+x_{\text {Boltzmann }} c_{1}
$$

If several absorption features are utilized, a Boltzmann plot analysis can be performed using Equation 2.9. For each absorption feature observed, an ordered pair is formed by $x_{\text {Boltzmann }}$, the known lower state energy of each absorption feature, and $y_{\text {Boltzmann }}$, a function of the measured integrated area and the transition's spectroscopic parameters. The ordered pairs are plotted and a linear fit is performed, as is illustrated by Figure 2.1.

The resulting fit parameters, $c_{1}$ and $c_{0}$, and Equations $2.9,2.8 \mathrm{c}$, and $2.8 \mathrm{~d}$ can then be used to solve for the temperature and number density assuming ro-vibrational equilibrium. The Boltzmann-plot method for calculating temperature from measured absorption spectra is a standard practice and has been utilized in similar TDLAS experiments of scramjet isolators and combustors. [37] 


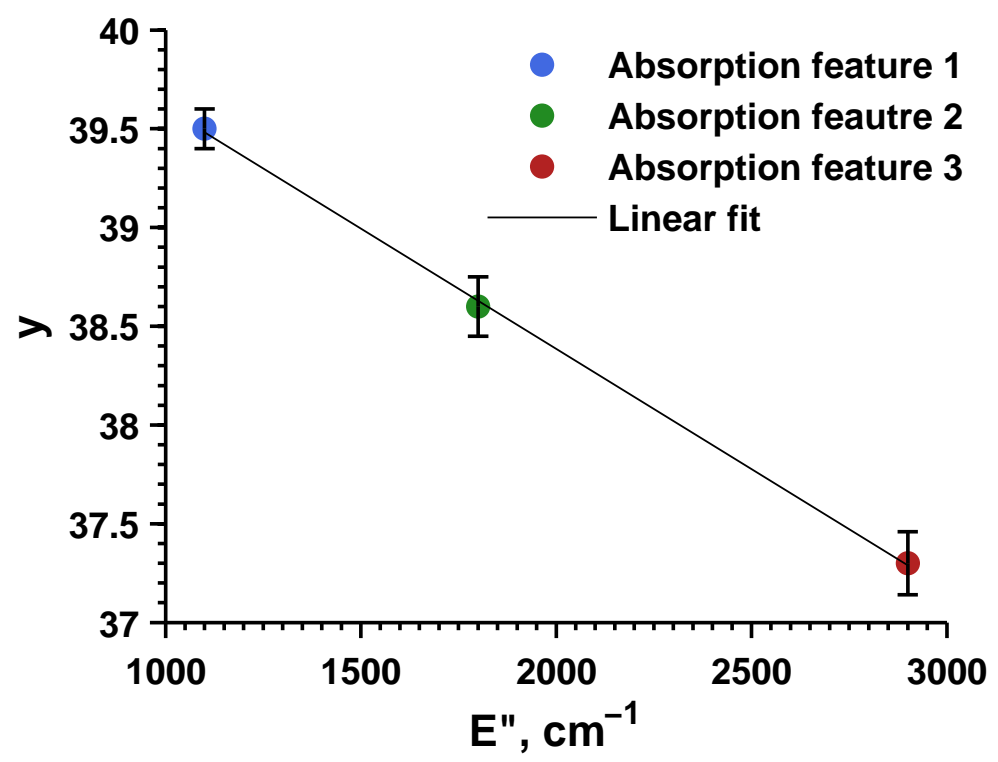

FIgURE 2.1: Example of Boltzmann plot analysis with three representative absorption features.

If only two absorption features are utilized, the Boltzmann plot method reduces to the well-known two-line thermometry expression, given by Equation 2.10:

$$
T=\frac{\frac{h c}{k_{B}}\left(E_{2}^{\prime \prime}-E_{1}^{\prime \prime}\right)}{\ln \left(\frac{A_{1}}{A_{2}}\right)+\ln \left(\frac{S_{2}\left(T_{r e f}\right)}{S_{1}\left(T_{r e f}\right)}\right)+\frac{h c}{k_{B}} \frac{E_{2}^{\prime \prime}-E_{1}^{\prime \prime}}{T_{r e f}}}
$$

After the temperature and water vapor number density have been calculated, the water vapor mole fraction can be calculated via Equation 2.11. Though the pressure is not measured, both of the (sc)ramjet facilities considered in this research exhaust to atmosphere. The TDLAT measurements are acquired just downstream of the exit plane of both facilities, and thus a pressure of 1 atm will be utilized in Equation 2.11 to obtain the mole fraction of water vapor.

$$
X_{\mathrm{H}_{2} \mathrm{O}}=N_{\mathrm{H}_{2} \mathrm{O}} / N_{\text {total }}=\frac{N_{\mathrm{H}_{2} \mathrm{O}}}{\left(\frac{N_{A} P}{R T}\right)}
$$

where $X_{\mathrm{H}_{2} \mathrm{O}}$ is the water vapor mole fraction, $N_{\text {total }}$ is the total number density of the 
gas mixture, $N_{A}$ is Avagadro's number, $P$ is static pressure, and $R$ is the universal gas constant.

\subsubsection{Computed Tomography Theory}

Computed tomography is a method in which an object (or field) is mathematically rendered from projections of the object. The mathematical solution of how to reconstruct a function from a series of projections was proposed by Radon in 1917, while the invention of the "modern" x-ray computed tomographic scanner was first introduced by Hounsfield in 1972. [38] Tomographic reconstruction techniques have a wide range of application and are employed in many scientific fields including physics, chemistry, astronomy, geophysics, and medicine. [39][40][41][42] The tomography process is most commonly associated with the medical field's Computed Axial Tomography (CAT) scan.

In the case of TDLAT reported herein, the object which is studied, and subsequently reconstructed, is the distribution of water vapor in the exhaust of a scramjet model. The projections of the object are the line-of-sight (LOS) absorption measurements. There are several methods of tomographic image reconstruction but these can generally be categorized into two groups: algebraic reconstruction techniques (ART) and transform-based methods. The transform-based method of filtered back projection has been used for tomographic inversion in TDLAT analysis codes at the University of Virginia in the past through the use of a MATLAB Image Processing Toolbox script ifanbeam.m. [43][44][31] While this script provides the advantage of being commercially available, it also severely limits the user's control over critical variables.

Algebraic reconstruction techniques (ART) are iterative in nature and are known as Kaczmarz methods in numerical linear algebra. [45] The problem which is encountered in a physical measurement can be described using finite series expansion techniques and expressed as a system of linear equations depicted in Figure 2.2 and 
given by Equation 2.12:

$$
\sum_{j} A_{i j} x_{j}=\hat{P}_{i}, \mathrm{i}=1 \text { to } N_{\text {rays }}, \mathrm{j}=1 \text { to } N_{\text {pixels }}
$$

where $A_{i j}$ is a matrix corresponding to the area which the $i^{\text {th }}$ ray intersects with $j^{\text {th }}$ cell, $x_{j}$ corresponds to the reconstruction variable at the $j^{\text {th }}$ cell (in this case absorbance), and $\hat{P}_{i}$ is the measured LOS projection (also called a ray sum or sinogram element) for the $i^{t h}$ ray. It is important to note that here all of the pixels in an $m \times n$ matrix are simply assigned an index, $j$, which is different than the customary $i, j$ description of a point in a matrix.

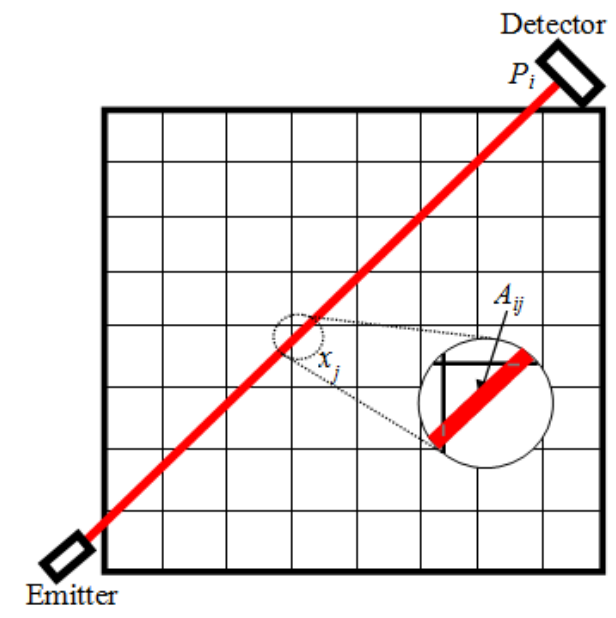

Figure 2.2: Algebraic Reconstruction Technique: finite series expansion of discretized measurement. [31]

Maximum-Likelihood Expectation-Maximization (ML-EM) is a particular form of ART which involves the maximization of an objective function. Verkruysse and Todd [46] utilized ML-EM for tomographic reconstruction of chemical concentrations in the atmosphere. This algorithm can be used for tomographic inversion in the TDLAT technique, with the reconstruction variable being absorbance, seen in Equation 2.1, in place of Verkruysse and Todd's concentration, denoted by $x$. The scheme is organized as follows: 
1. $\hat{P}_{i}$, the measured projections or "ray sums", are known. These projections are organized in matrix termed a sinogram.

2. $A_{i j}$, the matrix which geometrically relates how much of the $i^{\text {th }}$ ray intersects with the $j^{\text {th }}$ cell, is calculated.

3. The absorbance values, $x_{j}$, which satisfy $p_{i} \approx \hat{P}_{i}$ are iteratively solved for, where $p_{i}$ are the computed re-projections are $\hat{P}_{i}$ are the measured projections. Therefore, new calculated re-projection values are iterated upon until the calculated re-projection is approximately equal to the measured projection.

To initialize the process, first an initial guess of the absorbance distribution, $x_{j}^{q=0}$, is formed by guessing an average value of $x$ over the entire reconstruction domain. Next, an initial re-projection, $p_{i}{ }^{q=0}$, is calculated by:

$$
p_{i}^{q=0}=\sum_{j} A_{i j} x_{j}^{q=0}
$$

The absorbance distribution $x_{j}$ is then updated with each iteration of the ML-EM code according to Equation 2.14. [46]

$$
x_{j}^{q+1}=\frac{x_{j}{ }^{q}}{\sum_{i} A_{i j}} \sum_{i} \frac{\hat{P}_{i}}{p_{i}{ }^{q}} A_{i j}
$$

Next, a re-projection is calculated from the most recent absorbance distribution $x_{j}^{q=1}$ by:

$$
p_{i}^{q=1}=\sum_{j} A_{i j} x_{j}^{q=1}
$$

The resulting re-projection $p_{i}$ is then compared to the measured projection $\hat{P}_{i}$ and the process is repeated with $q=q+1$ until the difference between the two is minimized. When the solution converges, $x_{j}^{q+1}$ is the resulting absorbance distribution. The MLEM algorithm preserves absorbance throughout the process in both the projections 
and the absorbance distribution.

\section{$2.3 \quad$ Experimental Overview}

\subsubsection{Hardware Design}

There are two basic geometries which can be used to collect the individual TDLAS measurements which comprise a full TDLAT data set: fanbeam and parallel beam, as is illustrated in Figure 2.3. In the fanbeam configuration, seen in Figure 2.3a, the infrared laser beams originate from vertices, $s_{i}$, and traverse the measurement region which contains a distribution, $O$. The vertex locations are characterized by their angle relative to the y-axis in the reference Cartesian plane, $\theta_{f_{i}}$, and their (constant) distance to the origin of the reference Cartesian plane, $r$. The angle formed between two consecutive fans is defined as $\Delta \theta$. The rays are formed by pivoting the laser source about $s_{i}$ by an angle $\Delta \alpha$ several times. The total inscribed angle by the fanbeam is $\alpha$, equal to $\Delta \alpha \times\left(N_{\text {rays }}-1\right)$. In the parallel beam configuration, shown in Figure $2.3 \mathrm{~b}$, the infrared diode laser again revolves around the distribution of interest, $O$, at a constant radius, $r$. At discrete and evenly spaced locations along its circular path, $s_{i}$, the laser source stops and translates linearly in uniform increments, $\Delta \alpha$, traversing the laser beam across the measurement space.

For the research presented herein, data will be collected in a fanbeam geometry, the experimental parameters of which are listed in Table 2.1. The fanbeam geometry has been selected due to the simplicity of rotation about a vertex, enabled by a single rotational stage with a small footprint, rather than a larger linear translation stage to be used for the parallel beam geometry. The rotation of the vertex $360^{\circ}$ around the measurement area, for both the fanbeam and parallel beam geometries, would be achieved by a large rotational stage. The experimental fanbeam parameters differ slightly for the two facilities measured at, the University of Virginia's supersonic combustion facility (UVaSCF) and NASA Langley's direct-connect supersonic 
combustion test facility (DCSCTF). These experimental facilities will be discussed in detail in Chapters 4 and 5.

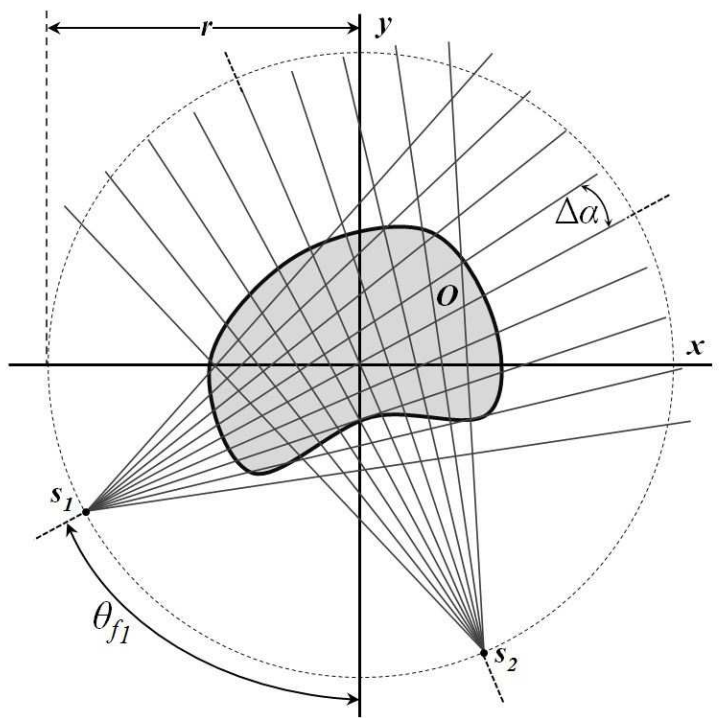

(a)

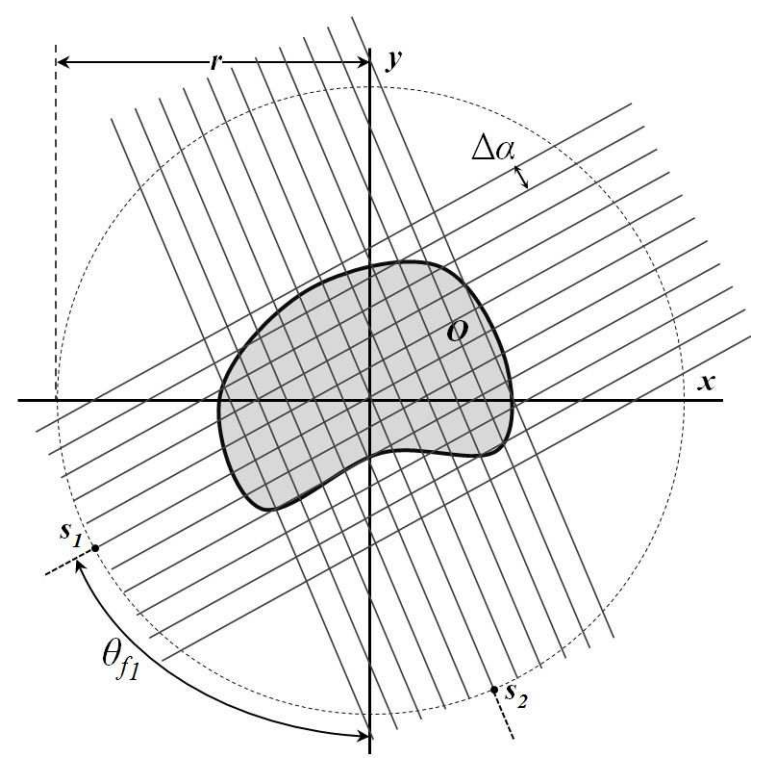

(b)

Figure 2.3: (a) Fanbeam style geometry, and (b) parallel beam style geometry.

TABLE 2.1: Fanbeam geometry parameters for both test facilities utilized.

\begin{tabular}{lllllll}
\hline Facility & $\Delta \theta$ & $\Delta \alpha$ & $\alpha$ & $N_{\text {fans }}$ & $N_{\text {rays }}$ & $r, \mathrm{~mm}$ \\
\hline UVaSCF & $9.0^{\circ}$ & $0.5^{\circ}$ & $17^{\circ}$ & 40 & 35 & 203.2 \\
NASA Langley DCSCTF & $9.0^{\circ}$ & $1.3^{\circ}$ & $26^{\circ}$ & 40 & 21 & 442 \\
\hline
\end{tabular}

Tomographic data is typically organized into a matrix termed a sinogram. The sinogram is arranged with each column representing a fanbeam, and each row corresponding to the individual rays within the fanbeam, as seen in Figure 2.4. In the TDLAT application, each individual ray results in a single value of integrated absorbance for each line in a scan. Therefore, the resulting sinogram (for each individual absorption feature) represents the integrated absorbance values for each of the rays. The total number of rays collected is equal to the number of fans $(360 / \Delta \theta)$ multiplied by the number of rays per fan, $N_{\text {rays }}$, which equals either 1400 or 840 in this research, for the datasets collected on the UVaSCF and the NASA Langley DCSCTF respectively. 


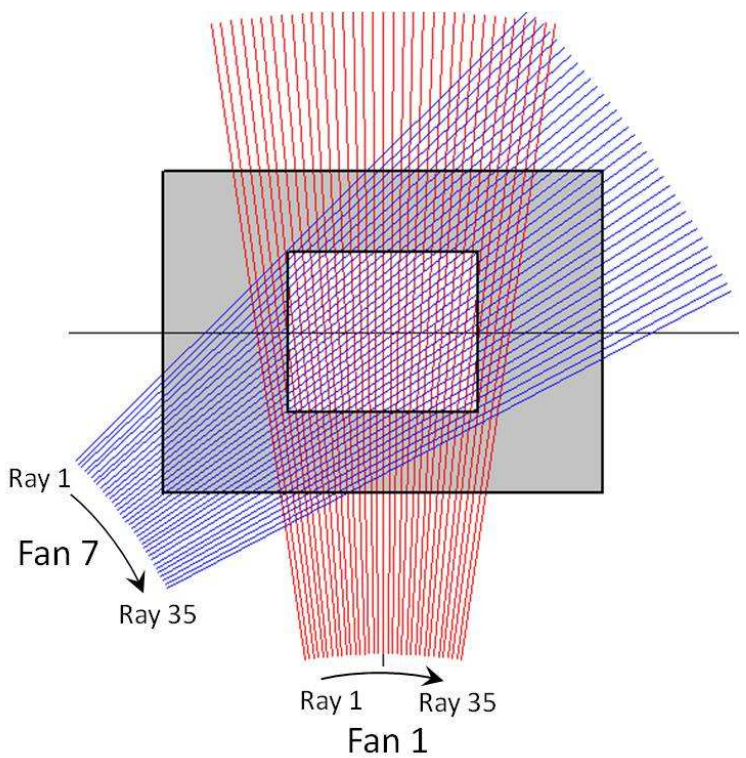

(a)

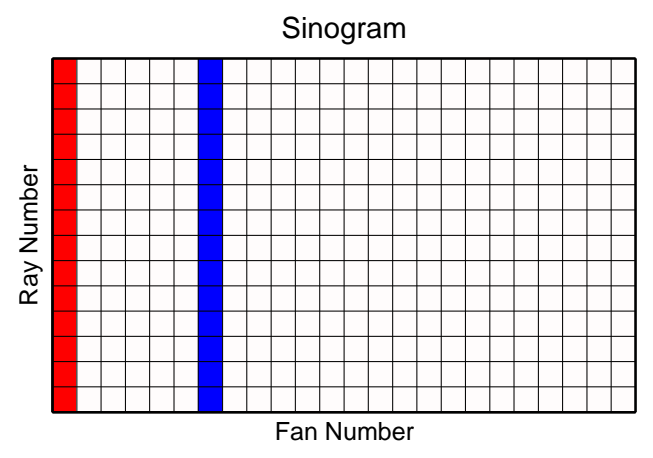

(b)

FIGURE 2.4: Orientation of fanbeam collection geometry in both (a) measurement space, and (b) sinogram space.

\subsubsection{Optical Design}

The TDLAT technique makes use of three NTT Electronics polarization maintaining (PM) fiber coupled distributed feedback (DFB) lasers $(1 \times$ NLK1E5GAAA and $2 \times$ NLK1B5EAAA). The selection of the line transitions to use in this research was guided by X. Zhou et al. [47] and consisted of four main criteria:

1. Sufficient absorption required for high signal-to-noise (SNR) measurements

2. Transitions should be selected to minimize interference from ambient (low-temperature) $\mathrm{H}_{2} \mathrm{O}$, i.e. $E^{\prime \prime} \geq 1700 \mathrm{~cm}^{-1}$ for the selected transitions.

3. The transitions must have sufficiently different lower state energies, $E^{\prime \prime}$, to yield a peak height ratio that is sensitive to temperature (in the range $1000-2500 \mathrm{~K}$ ), i.e. $\left|E_{i}^{\prime \prime}-E_{i+1}^{\prime \prime}\right| \geq 700 \mathrm{~cm}^{-1}$.

4. The selected transitions must be isolated from nearby transitions.

The transitions selected for use this experiment are given in Table 2.2. Note that the designations of the absorption transitions used in this research are used only for convenience in referencing the transitions. The four transitions identified in Table 
2.2 were found to have sufficiently favorable characteristics for this research based on the aforementioned criteria. However, they were inaccessible within a single laser scan. For this reason, three lasers were used. KB1 \& KB2 are best suited for a lower temperature range, and KB2, KB3, and KB4 are best suited for a higher temperature range. Note that KB3 and KB4 are located very near to each other and are accessible in a single scan. Therefore, a total of three lasers, with four probed transitions, will be utilized for the research reported herein.

TABLE 2.2: Transitions used in TDLAT system and their spectroscopic parameters. [16][48]

\begin{tabular}{lllll}
\hline Transition & $\begin{array}{l}\text { Line center Wavenumber } \\
\nu_{0} \\
\left(\mathrm{~cm}^{-1}\right)\end{array}$ & $\begin{array}{l}\text { Transition Reference Intensity } \\
S_{\text {ref }} \\
\left(\mathrm{cm}^{-1} / \text { molecule }-\mathrm{cm}^{-2}\right)\end{array}$ & $\begin{array}{l}\text { Lower State Energy Level } \\
\left(\mathrm{cm}^{-2} / \mathrm{atm}\right)\end{array}$ & $\begin{array}{l}\mathrm{cm}^{\prime \prime} \\
\left(\mathrm{cm}^{-1}\right)\end{array}$ \\
\hline $\mathrm{KB} 1$ & 7185.59 & $7.865 \times 10^{-22}$ & $1.95 \times 10^{-2}$ & 1045.06 \\
$\mathrm{~KB}{ }^{*}$ & $7444.35 / 7444.369 / 7444.37$ & $4.70 \times 10^{-23}$ & $1.10 \times 10^{-3}$ & $1774.8 / 1806.67 / 1806.67$ \\
$\mathrm{~KB} 3$ & 7472.22 & $1.016 \times 10^{-25}$ & $2.52 \times 10^{-6}$ & 2981.36 \\
$\mathrm{~KB} 4$ & 7471.617 & $7.96 \times 10^{-26}$ & $1.97 \times 10^{-6}$ & 2952.39 \\
\hline
\end{tabular}

${ }^{*}$ This transition consists of three transitions spaced $.02 \mathrm{~cm}^{-1}$ apart. They are essentially indistinguishable at the test conditions reported herein.

Figure 2.5a shows each absorption feature's line strength as a function of temperature within a range of expected experimental temperatures. Figure 2.5b shows the ratio of the temperature-dependent line strengths for several line pair combinations of the four absorption features. 


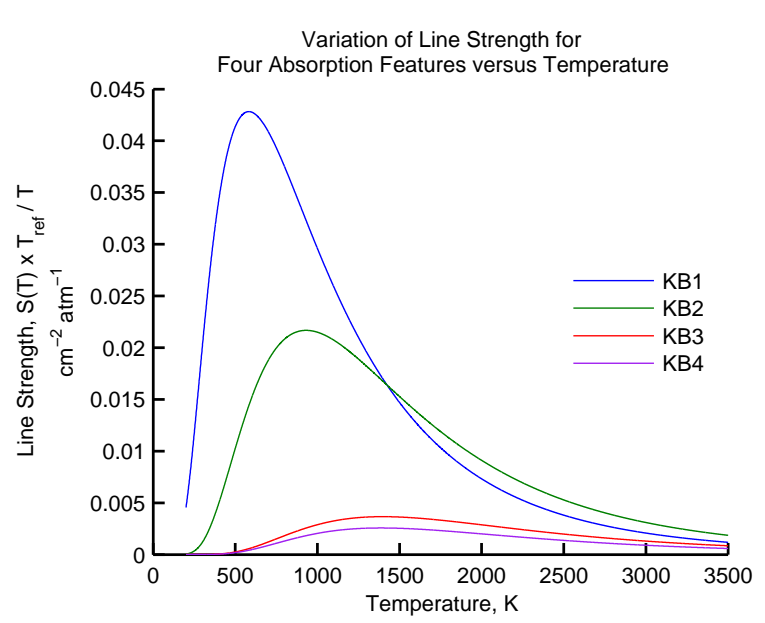

(a)

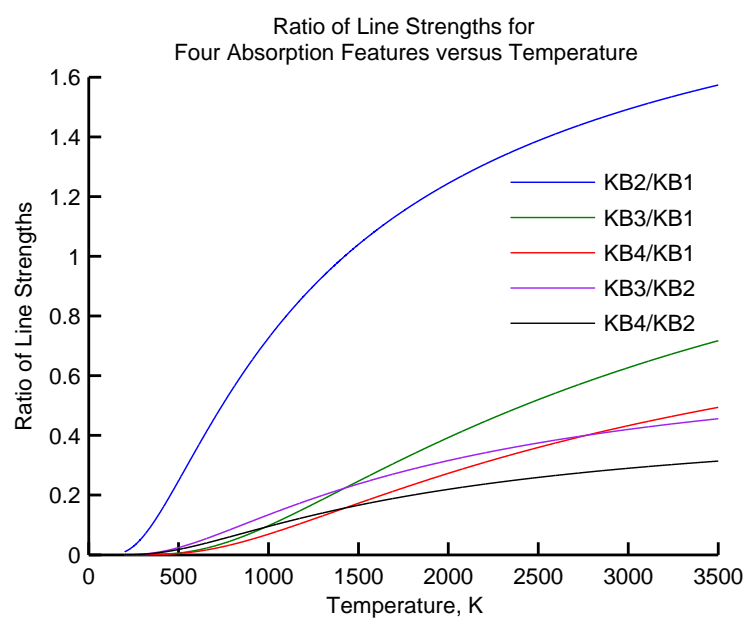

(b)

Figure 2.5: (a) Temperature-dependent line strength of the four selected absorption features, and (b) ratio of temperature-dependent line strengths for various line pairs.

Data are collected with all three lasers, in a time-multiplexed mode, for the full duration of the experiment. The lasers were temporally multiplexed using a MEMStype fiber optical switch system. While the KB1 laser is switched on and scans over the first transition, the KB2 and KB3 \& KB4 lasers are switched off. After KB1 has scanned over its prescribed range, it switches off and KB2 is switched on. Lastly, KB2 is switched off and the laser probing KB3 and KB4 is switched on. This process is repeated each time a measurement is collected and is seen in Figure 2.6. In order to make the measurements as close to simultaneously as possible, each trio of laser scans is accomplished in $10 \mathrm{~ms}$. 


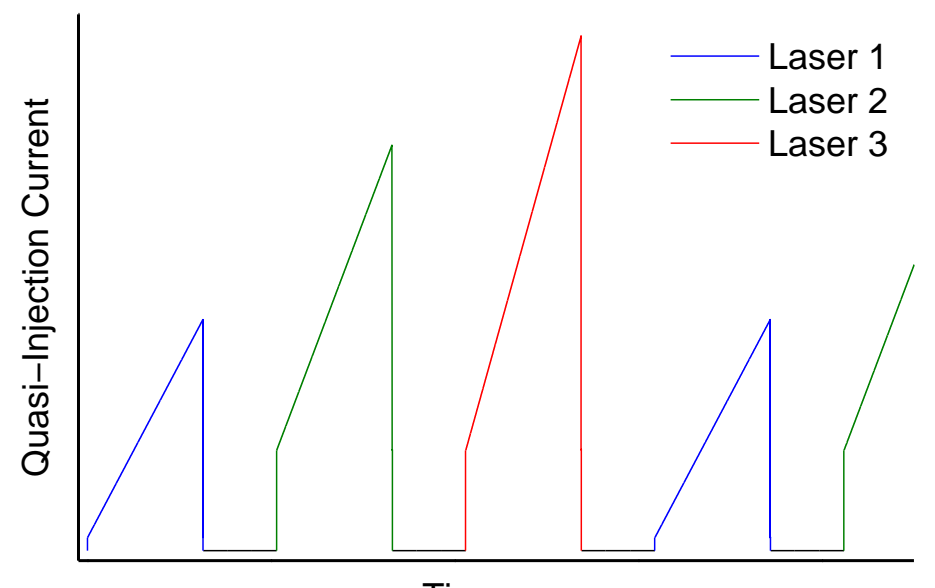

Time, ms

FIGURE 2.6: Illustration of three temporally multiplexed lasers. Not to scale.

The optics for the TDLAT system are housed in a Tomographic Emitter-Detector (TED) box. The TED box optics and design have undergone several iterations during the development of the TDLAT technique. The first iteration, TED 1.0, was designed by Bryner [31] and Snyder [49]. Seen in Figure 2.7, the IR light was brought to the TED box through a single mode (SM) optical fiber, where the light passed through a collimating optic and was reflected $90^{\circ}$ via a mirror. The collimated light then traversed through a beam sampler, where a small portion of the beam was picked off. This small portion of the signal was detected and recorded so that real-time background intensity fluctuations could be accounted for in the measured TDLAS signal. The majority of the beam continued on its original path, traversing through a mirror positioned at $45^{\circ}$ to the beam path via a hole through the center of the mirror. The beam then exited the TED box through a purged tube and out across the measurement space. The beam then impinged on a retroflective surface, which was a metallic arc covered with 3M Scotchlite high gain reflective sheeting 7610 . The retroreflector remained stationary while the TED box rotated through the fanbeam, and therefore the curved surface of the retroreflector ensured that the path length of every ray, from the TED box to the retroreflector and back again, was constant. As the beam reflected upon its original path, it began to conically diverge. When 
the diverging beam re-entered the TED box, the portion which had diverged enough impinged on the mirror with the hole and through a bandpass filter onto a detector. The TED box and retroreflector volumes were purged with nitrogen to minimize ambient water vapor absorption.

Difficulties with this TED box design mainly stemmed from the use of Thorlabs construction cubes, which limited the flexibility of the positioning of the optical components. Alignment of the beam was performed mainly by adjusting the first $45^{\circ}$ mirror. Alignment with a single mirror was often undesirable, as it had the ability to significantly alter the beam's path with no other means of correction. The method of using a mirror with a hole in it to pass the out-going beam and reflect the in-coming beam was adequate, but slight misalignment of the outgoing beam would result in a significant loss of measured signal.

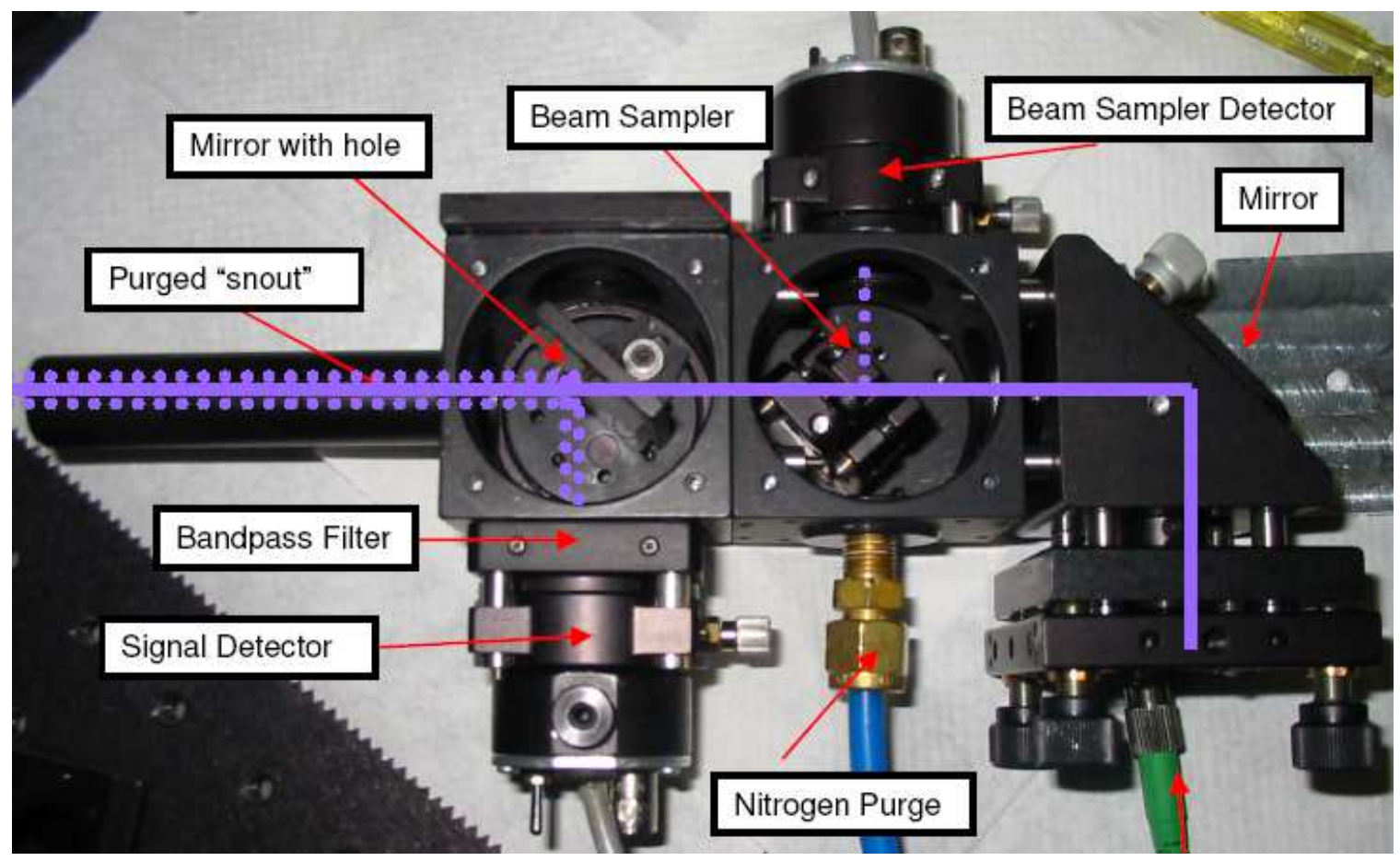

FiguRE 2.7: First iteration of the optical TED box for TDLAT experiment, designed by Bryner and Snyder. [31]

The second iteration of the TED box was designed by the author for use in the NASA Langley measurement campaign and is seen in Figure 2.8. It is readily appar- 
ent that the design of TED 2.0 was no longer assembled using Thorlabs construction cubes. This design eliminated the spatial limitations from the TED 1.0 design, but instead required more custom machining. In place of the mirror with the hole, a reflective right-angle prism was utilized. The near infrared light was brought into the box through a polarization maintaining (PM) fiber. The use of the PM fiber made this system more robust than the previous iteration, which was very sensitive to the movement and rotation of the TDLAT system. The beam was then immediately passed through a collimating lens, collimating the beam diameter to $1 \mathrm{~mm}$. It then passed through an aperture slightly larger than $1 \mathrm{~mm}$. The collimated beam traveled past the small reflective right-angle prism, and finally out another aperture before it exited the TED box. It then traveled through the test gas, impinged on the retroreflector, and was reflected upon its original path, diverging slightly. The spreading beam passed again through the test gas and back into the TED box through an aperture. The beam's diameter was larger than previously, and half of the spread beam impinged on the reflective right-angle prism. This portion of the beam was then directed through a bandpass filter (to filter out flame luminosity), a lens, and finally onto an InGaAs detector.

Figure 2.8a depicts how the reflective right-angle prism was mounted using a cantilevered arm, attached to a Thorlabs KS05 kinematic mirror mount. This allowed for tip/tilt of the mirror, but translation of the cube mirror toward or away from the detector (and thus moving in relation to the outgoing and incoming beam path) would require the entire cantilever arm to be translated. As can be seen in Figures $2.8 \mathrm{~b}$ and 2.8c, the $1 \mathrm{~mm}$ aperture was positioned within a Thorlabs HPT1 XY translating lens mount. This coated aperture was intended not to impede the outgoing beam, but instead to absorb the returning IR light which has not been deflected by the reflective right-angle prism. Without the presence of this aperture, it was possible that an interference pattern would form via the optical cavity formed between the collimating lens and the retroreflector. The detector utilized was a Thorlabs PDA10CS InGaAs 
transimpedence amplified photodetector with switchable gain. To create the fanbeam as mentioned previously, the TED box was mounted to a Velmex rotational stage, allowing it to rotate through an entire fan. The TED box and its lid were coated with Aeroglaze Z306, an absorptive polyurethane paint, to minimize unintentionally scattered IR light. The optics enclosed within the TED box were purged with dry air, along with the volume surrounding the retroreflector. 


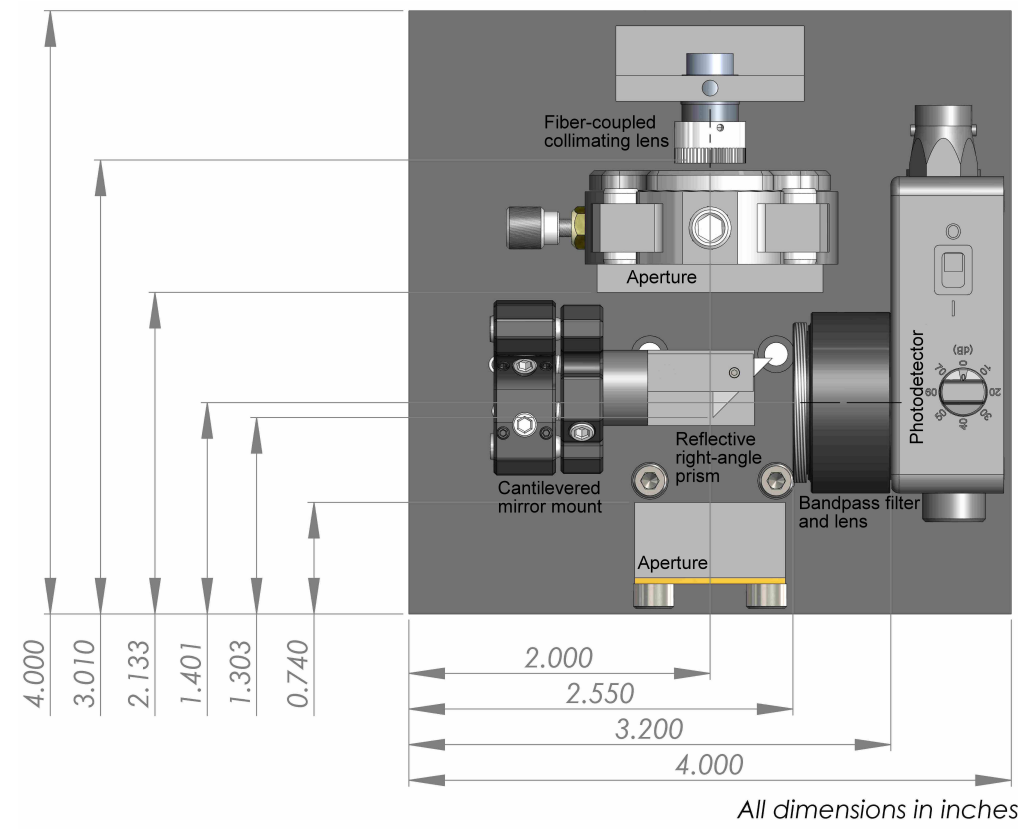

(a)

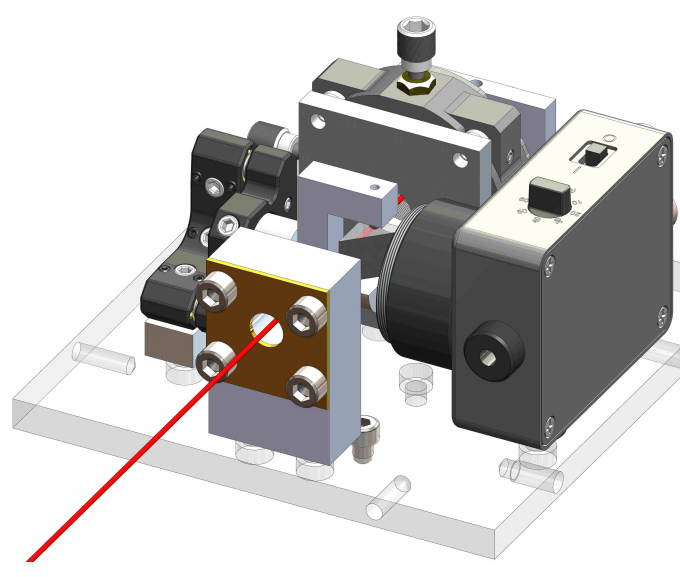

(b)

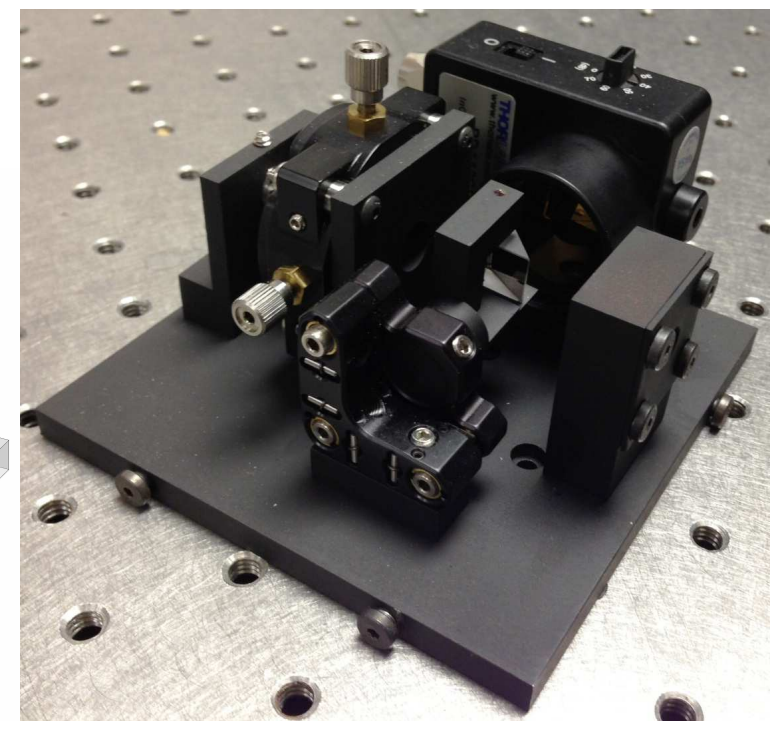

(c)

FiguRE 2.8: TED box 2.0 for use in measurements on NASA Langley DCSCTF (a) in dimensioned SolidWorks model, (b) SolidWorks isometric view, and (c) fabricated and coated in absorptive Aeroglaze paint. Solid models of Thorlabs components property of Thorlabs, Inc. 
A third iteration of the TED box was carried out for measurements performed at the UVaSCF. Seen in Figure 2.9, the changes from TED 2.0 to 3.0 were relatively minor and mainly intended to improve the ease of alignment of the reflective rightangle prism and the apertures. Improvements were made to the mounting system for the reflective right-angle prism. The reflective right-angle prism is secured using a Thorlabs KM100C kinematic rectangular optic mount, which is mounted to a Thorlabs DT12 dovetail linear translation stage. This allows for the reflective right-angle prism to be fastened and aligned with the kinematic mount, but also independently translated into and out of the path of the laser beam. The bandpass filter utilized in the TED 3.0 is a Spectrogon BP-1365-084 nm bandpass filter and the lens is a Thorlabs C110TME-C aspheric lens with a focal length of $6.24 \mathrm{~mm}$, both affixed to the photodetector via a Thorlabs SM1 lens tube and pictured in Figure 2.10. The TED box is again coated with Aeroglaze Z306 to minimize scattered IR light and purged with nitrogen to minimize ambient water vapor absorption. 


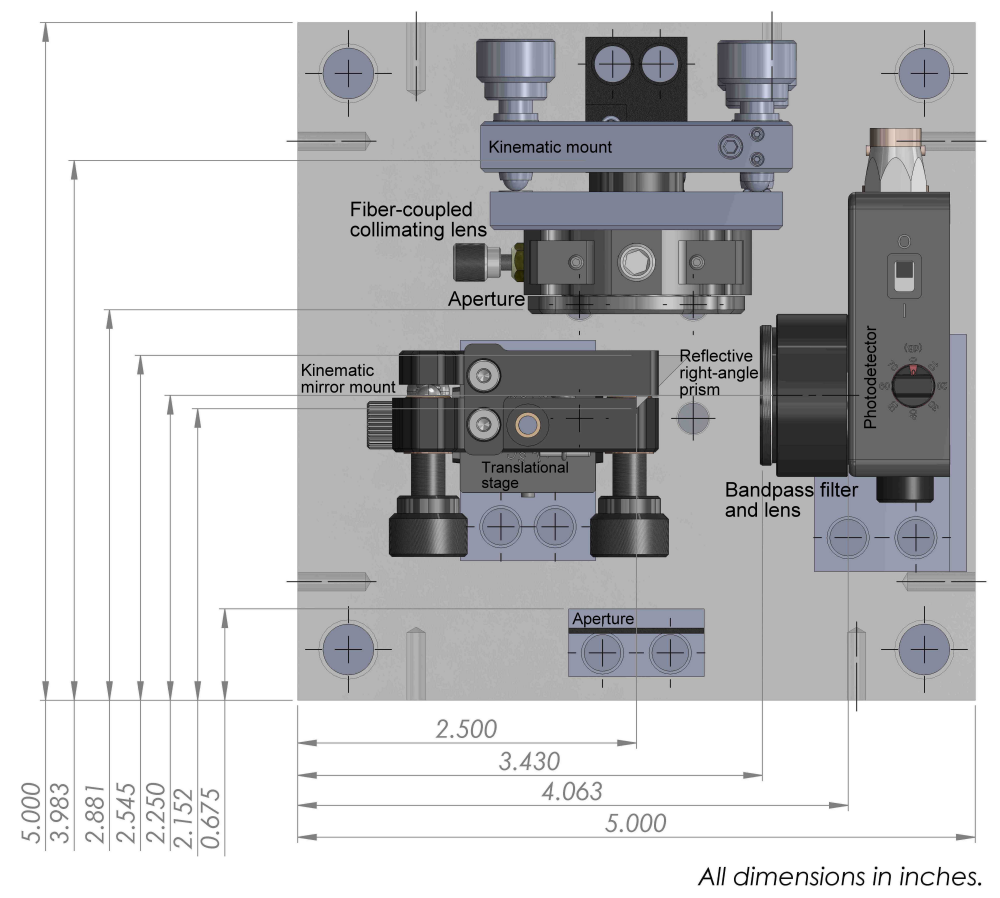

(a)

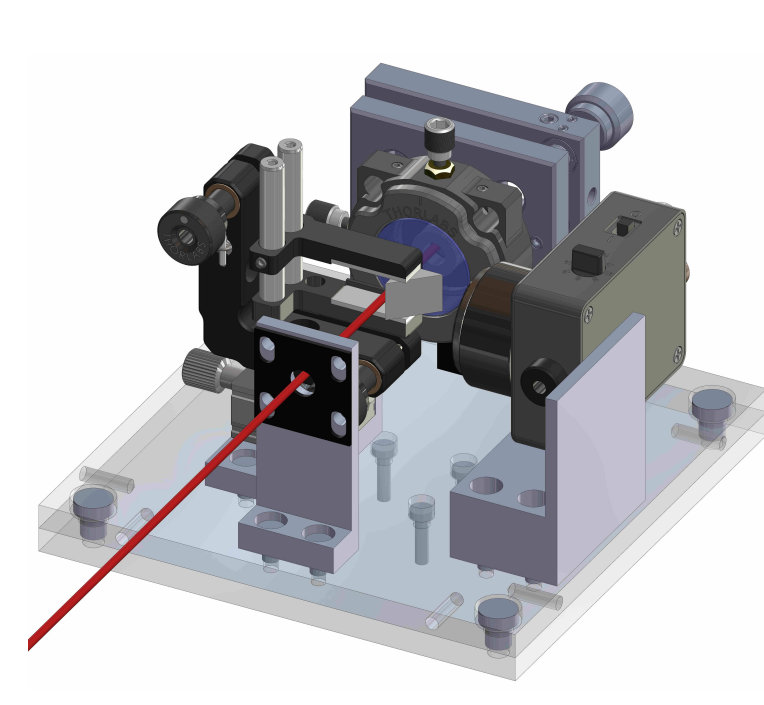

(b)

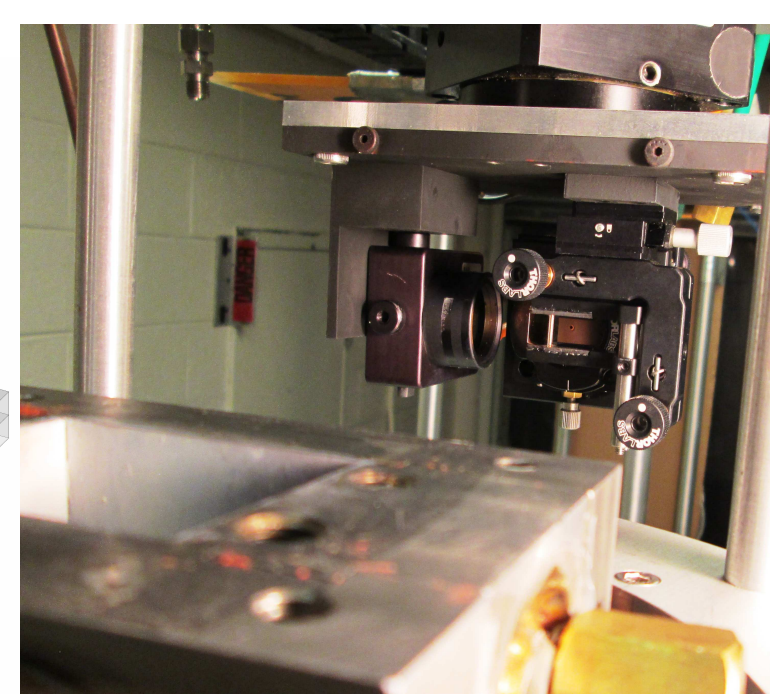

(c)

FIGURE 2.9: TED box 3.0 for use in measurements on UVaSCF (a) in dimensioned SolidWorks model, (b) SolidWorks isometric view, and (c) mounted at the exit plane of the UVaSCF. Solid models of Thorlabs components property of Thorlabs, Inc. 


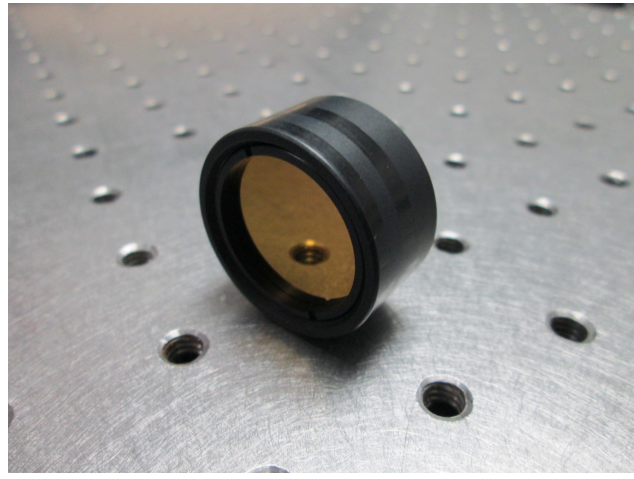

(a)

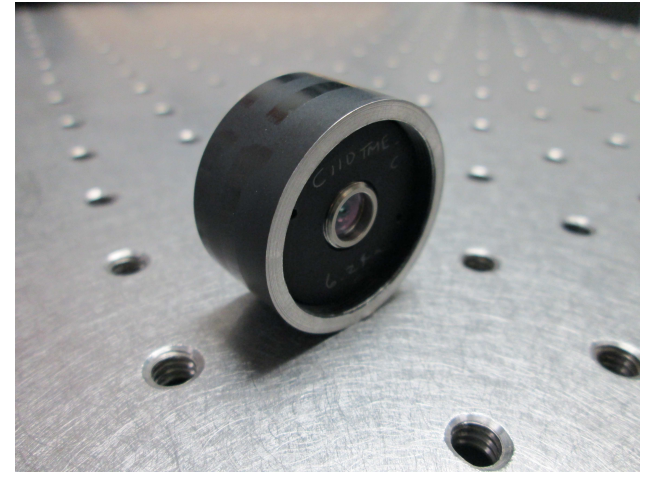

(b)

FIGURE 2.10: (a) Bandpass filter utilized in TED 2.0 and 3.0, and (b) aspheric lens utilized in TED 2.0 and 3.0.

\subsection{Three-phase Analysis}

The analysis of the TDLAT data takes place in three phases. The first phase transforms the raw measured voltage signals and processes this information to obtain the measured absorbance features and their spectrally integrated absorbances. The second phase utilizes the ML-EM algorithm to tomographically reconstruct the integrated absorbance distributions of the individual transitions. The third phase of analysis extracts the temperature and number density distributions from the spatially resolved absorbance distributions.

\subsubsection{Phase I}

Phase I begins with transforming the recorded raw voltage signals into absorbance profiles in frequency space. Each of the 1400 (or 840) LOS are treated individually

during Phase I. For each LOS, the entire recorded signal is multiplied by $\frac{20}{2^{16}}$ for visualization of the data with units of Volts (accounting for the $-10 \mathrm{~V}$ to $10 \mathrm{~V}$ range and 16 bit measurements). Next, the recorded signal is split into the three signals which comprise each LOS's data set: the laser diode current, the TDLAS signal (referred to as the signal for future reference), and the etalon signal (which contains the frequency information). An example of the first 2,000 points of these three raw signals is shown 
in Figure 2.11. Note that this measured signal is periodic and can be broken into 333-point segments, each of which corresponds to one of the three diode lasers. As described earlier, the three diode lasers are temporally multiplexed, meaning they are successively switched on and off. The pattern repeats and each of the diode lasers is measured 150 times per LOS, meaning a total of 450 of these 333-point segments seen in Figure 2.11 are recorded. The current signal seen in Figure 2.11 is not an actual measurement of the current, but is rather a synthetic signal recorded from the input signal to the laser controller. Therefore, while the magnitude of this current trace is nonsensical, the shape and sharp edges of the current are extremely meaningful and are used to identify the separation between the lasers switching on and off.

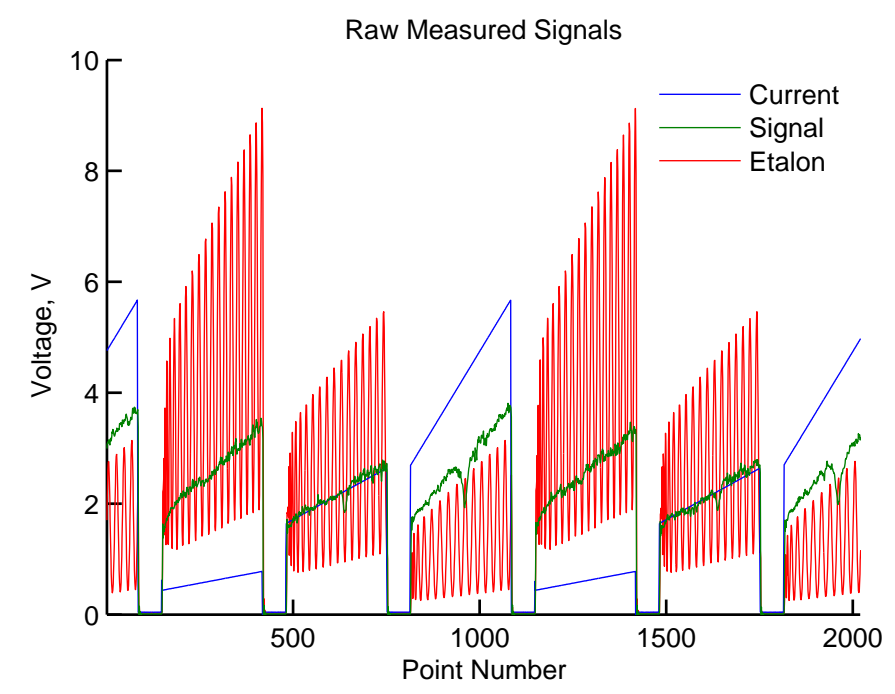

Figure 2.11: Example plot of raw measured signals: current, signal, and etalon data.

Next, the background intensity of the signal between each of the laser-on periods is determined. Seen in Figure 2.12a, the average voltage of the signal during the laseroff periods are determined and plotted as black circles. These average background values are typically very close to zero. Next, the variation in the background during each laser-on period is interpolated between the laser-off periods, and the resulting linear background values are subtracted for all 450 laser-on periods. Figure 2.12b shows the same signal as in 2.12a, but with the linear background removed.

After the background has been removed from each scan the signals are further 


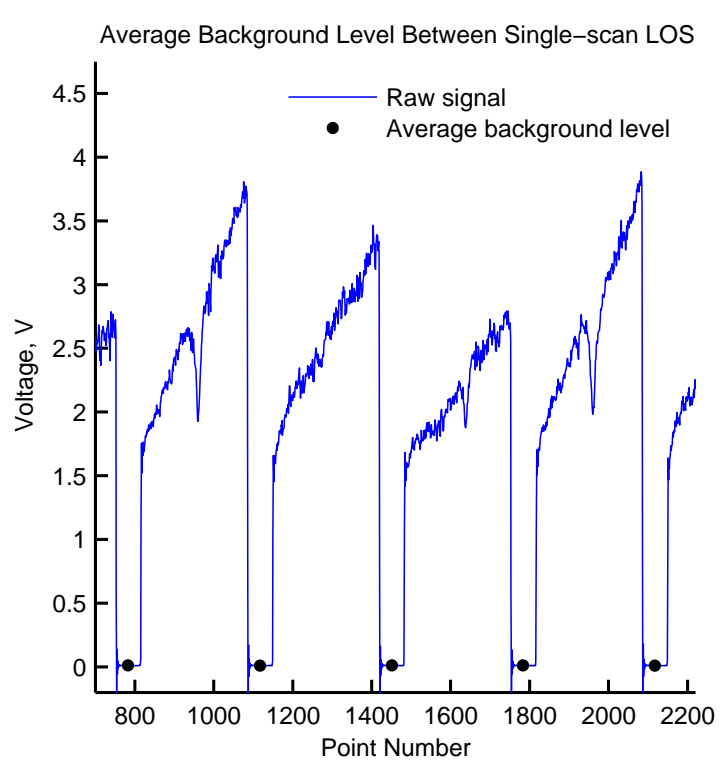

(a)

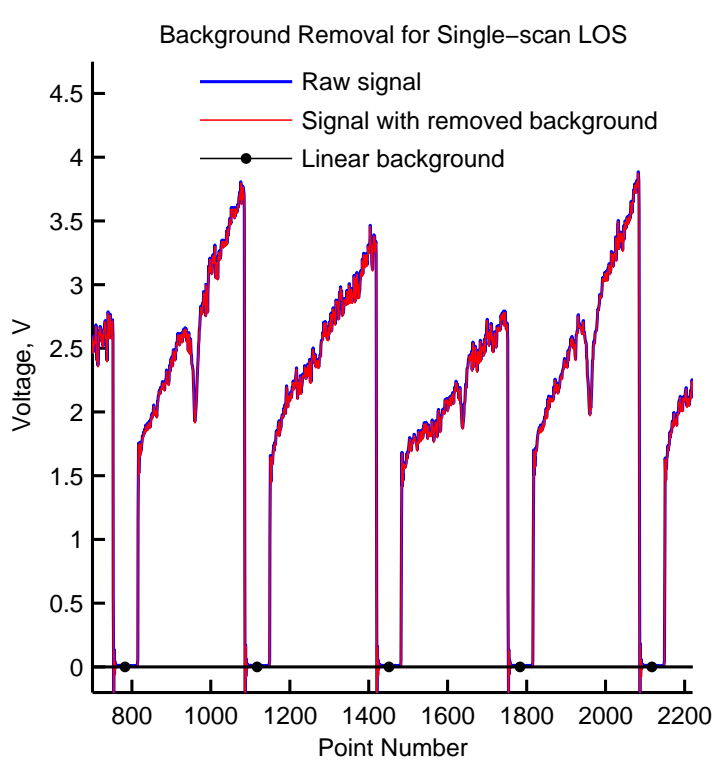

(b)

Figure 2.12: (a) An example of single-scan LOS signal and the identified average background level between laser-on periods, and (b) the same single-scan LOS signal with the linear background removed.

divided, corresponding to each of the three lasers. This results in nine total signals: a signal for each of the three lasers, an etalon trace for each of the three lasers, and a current trace for each of the three lasers. This separation and alignment of each of the signals is facilitated by aligning the current traces, made easier by their sharp rising and falling edges. Figure 2.13 shows an example of the mean and standard deviation of the 150 single-scan LOS for the KB1 absorption feature, its etalon trace, and current trace. Figure 2.14 shows a representative sample measurement of the three lasers (four transitions total, two of which occur within the third laser scan).

Next, the etalon signal is utilized to extract the relative frequency of the measured TDLAS signal. Pictured in Figure 2.15, a small portion of the original beam is split off and used to create an interference pattern. This small portion of the original light is aligned such that it is perpendicular to the planar faces of a BK7 glass cylinder, followed by a Thorlabs PDA10CS photo detector. As the frequency of the light is swept across the prescribed frequency range (to obtain the desired absorption features), the light travels through the etalon (glass cylinder), and at discrete frequen- 


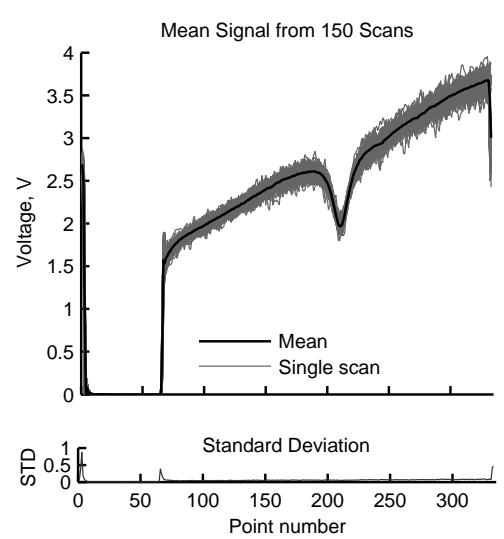

(a)

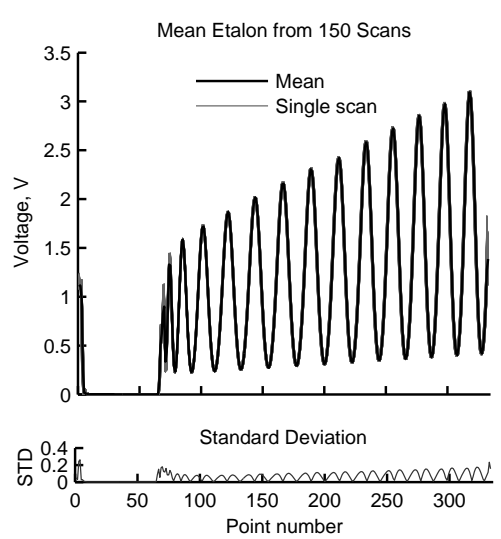

(b)

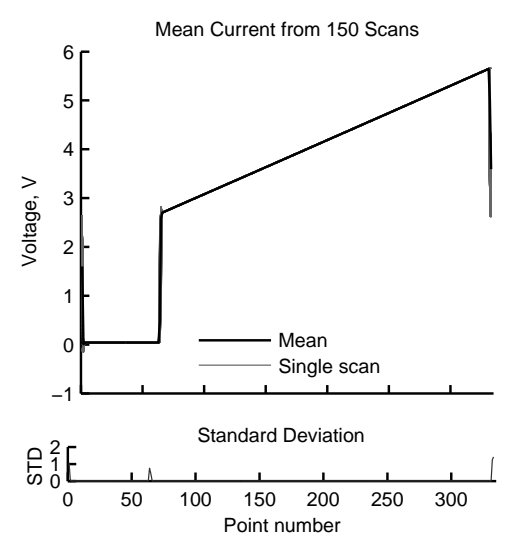

(c)

Figure 2.13: (a) An example of calculating the mean signal from 150 single-scan LOS, and (b) the mean etalon from 150 single-scan LOS, and (c) the mean current from 150 single-scan LOS.
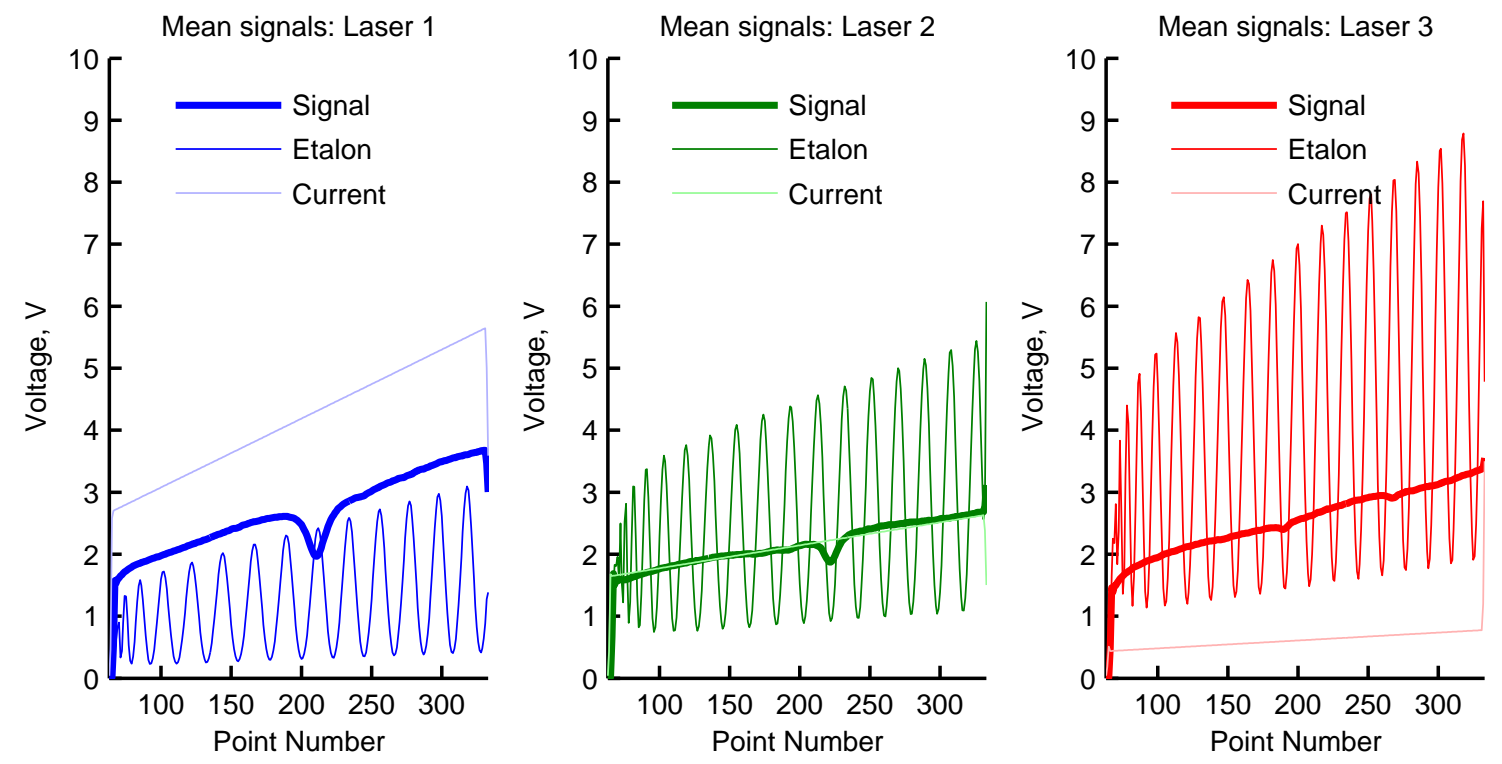

FIGURE 2.14: Representative signals for all three lasers after averaging over 150 single-scans. 
cies its wavelength is an integer multiplier of the path length within this cylinder. At these particular frequencies, the transmission of the light through the glass cylinder, and thus recorded onto the photo detector, is a local maximum. As the frequency is scanned, several of these transmission peaks are observed and the distance between the peaks is known as the free spectral range (FSR). The relationship between the observed FSR, the length of the cavity (the length of the cylinder $=.0254 \mathrm{~m}$ ), and the relative frequency of the light is given by Equation 2.16 [50]:

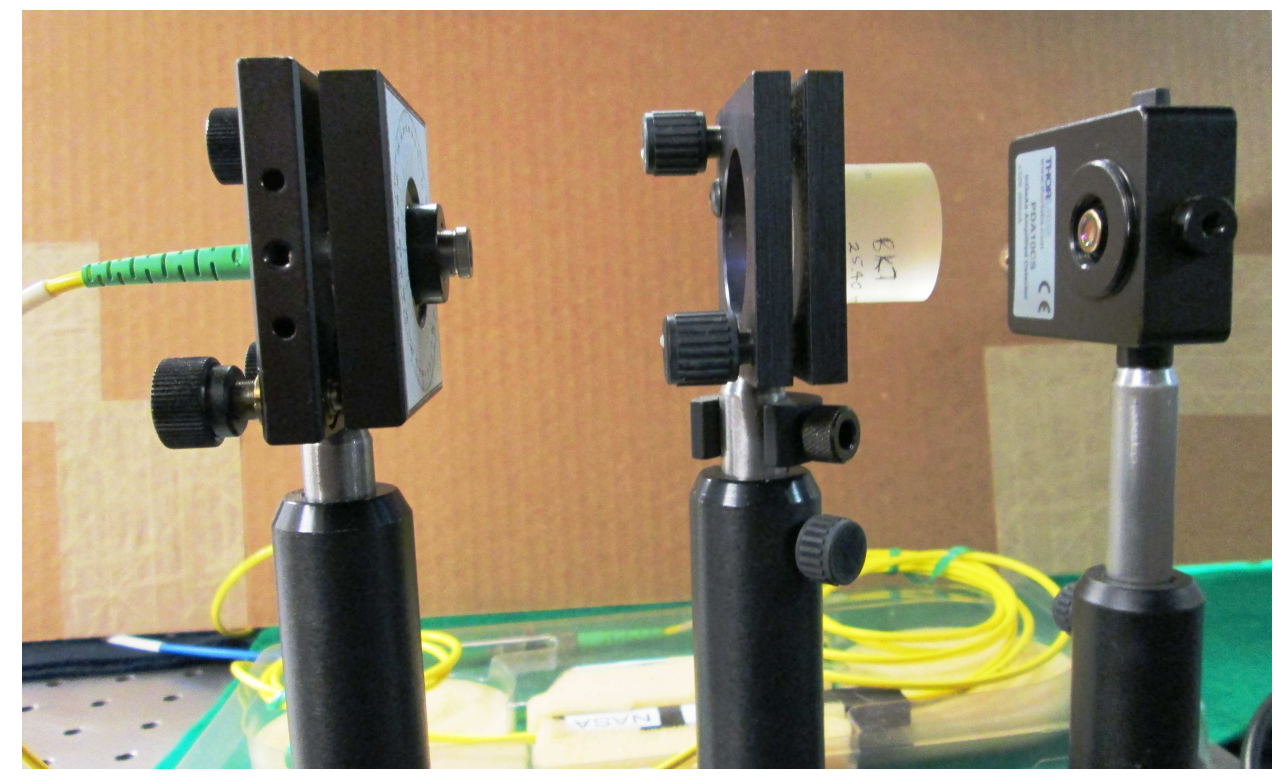

Figure 2.15: Optical setup for etalon detection and frequency retrieval.

$$
\Delta \nu_{f s r}=c /\left(2 \cdot n \cdot d_{f s r}\right)
$$

As is described by Barone [51] and seen in Figure 2.16a, the extrema of the etalon are identified using a MATLAB peak-finding routine. A polynomial is fit to these extrema and the etalon signal is divided by this polynomial to remove any potential shifting of the peaks due to the non-linearity of the injection current. The maxima of the leveled etalon are identified and are shown as the black points in Figure 2.16b. The spacing between each peak is calculated and using Equation 2.16 and the resulting 
relative frequency of each peak is obtained. A polynomial fit of the etalon peaks is performed such that a conversion between all point numbers and their relative frequencies is formed. An example of this frequency-mapping, performed for each of the three lasers and each LOS, is shown in Figure 2.16b. This allows for all future analysis of the recorded TDLAS signal to be performed in the frequency domain rather than in the point number domain (time-domain).

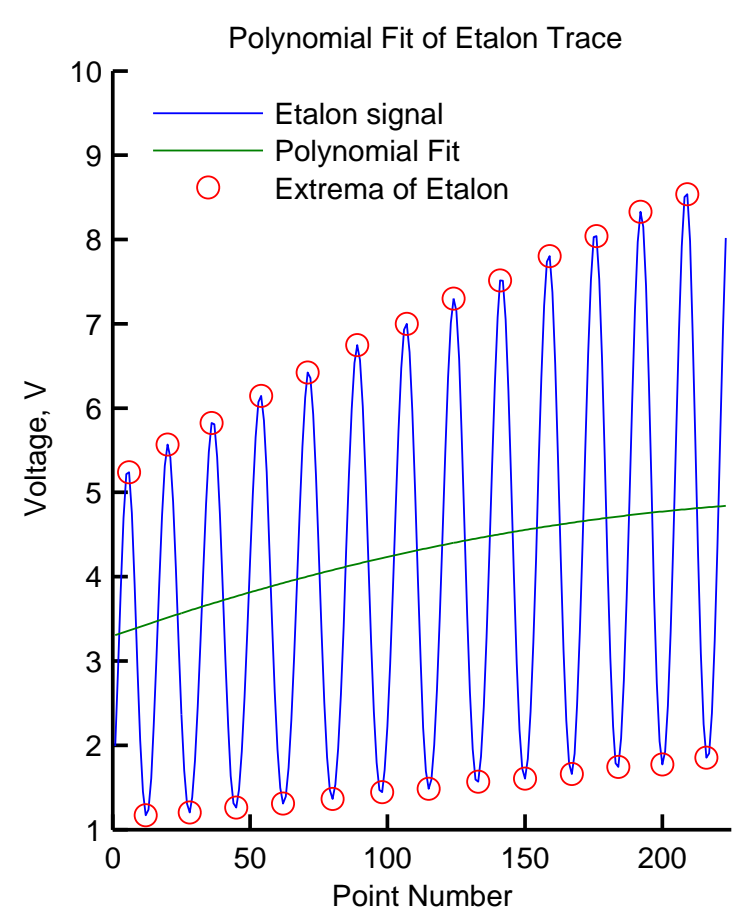

(a)

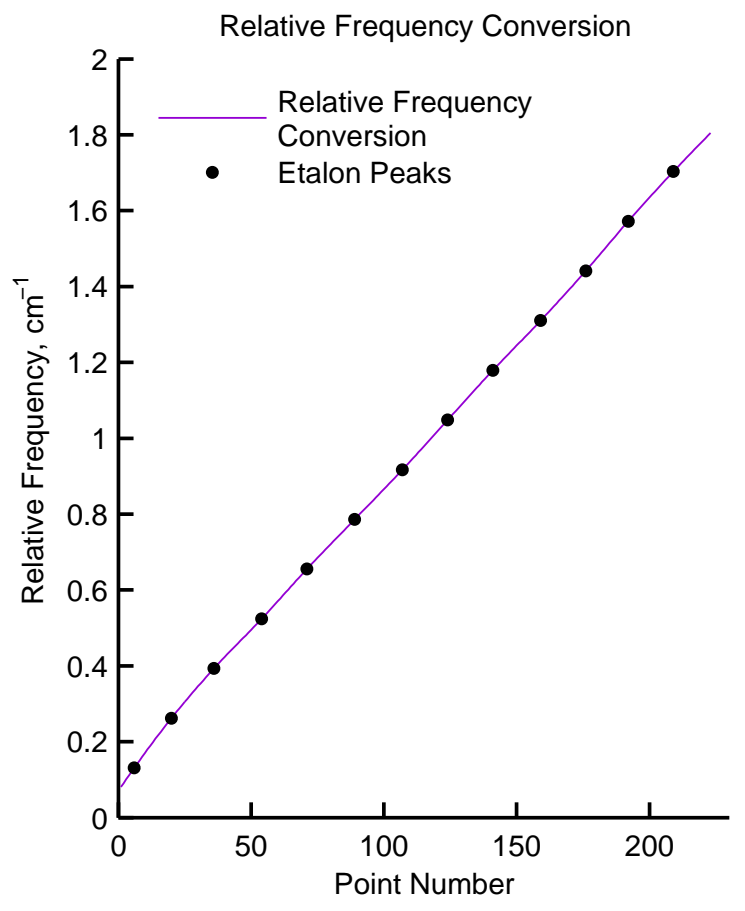

(b)

Figure 2.16: (a) An example of an etalon trace with the extrema identified, and (b) the resulting point number to relative frequency conversion.

After the previous steps have been accomplished for each complete data set (i.e. 1400 or 840 LOS), a database is formed which contains the mean signal and relative frequency for all three lasers. The next stage in the analysis focuses on the fitting of the TDLAS signals and extracting the integrated absorbance. The following analysis method is used for each of the three lasers (four absorption features), so the general method will be described just once, with results of each of the four absorption features shown. 
First, a preliminary fit of the baseline parameters is performed. As seen in Figure $2.17 \mathrm{a}$ (ii), the user is able to define regions to be masked from the baseline fit, generally the regions containing the absorption feature. The KB1 absorption feature has one main peak which falls between the red boundaries, but also a smaller neighboring peak masked within the green boundaries. The resulting signal, with masked absorption features, is fit using a polynomial with a user-defined order, generally $3^{\text {rd }}$ or $4^{\text {th }}$ order. The resulting polynomial fit is shown in Figure 2.17a(iii). This baseline represents the incident intensity of the light, $I_{0}$, as if there were no absorption. Therefore, the absorbance of the light can be calculated via Equation 2.1 as $-\ln \left(I / I_{0}\right)$ or $-\ln$ (measuredsignal/baseline) and seen in Figure 2.17a(iv). This figure is titled "Preliminary Absorbance" because a secondary baseline will be fit in the subsequent stage, which when incorporated into Equation 2.1 results in the true absorbance. The transmittance of the light, $T_{\nu}=I / I_{0}$, can also be calculated, and is shown in $2.17 \mathrm{a}(\mathrm{v})$ as $1-T_{\nu}$. This is the signal used for the ensuing Voigt fitting. It has been verified that the error incurred by fitting the $1-T_{\nu}$ signal, as opposed to the $-\ln \left(I / I_{0}\right)$ signal, is very minor (integrated absorbance typically varies by $<0.5 \%$ ). Figure 2.17b, 2.16c, and 2.16d show the baseline fitting procedure for the KB2, KB3, and KB4 absorption features, respectively. Note that the KB2 absorption feature has two neighboring transitions, while the KB3 and KB4 transitions do not have any near-neighbors masked in their baseline fits. 
(i)

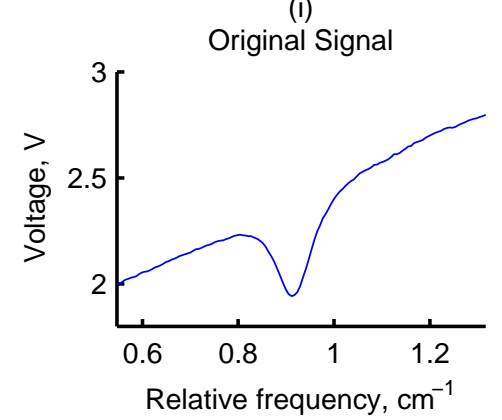

(iii)

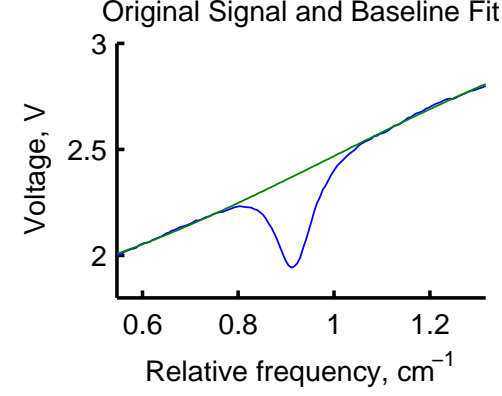

Relative frequency, $\mathrm{cm}^{-1}$

(i)

Original Signal

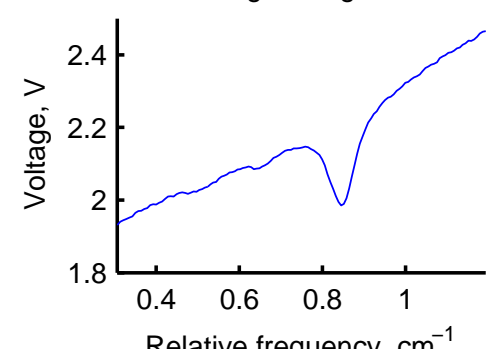

Relative frequency, $\mathrm{cm}^{-1}$

(iii)

Original Signal and Baseline Fit

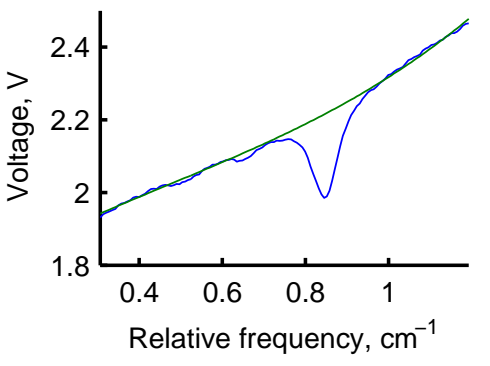

(ii)

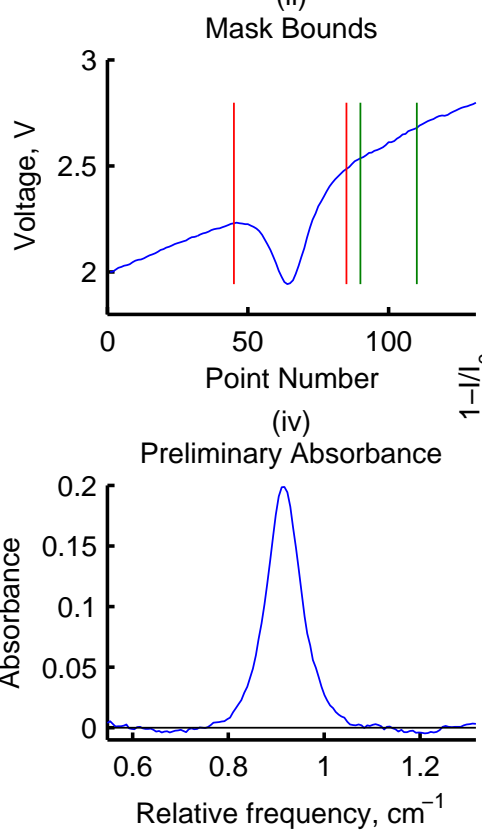

(a) (v)

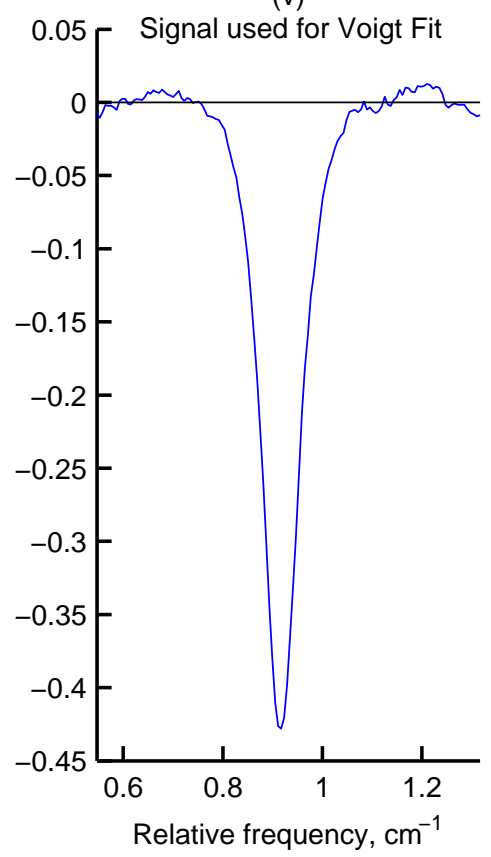

(v)

(ii)
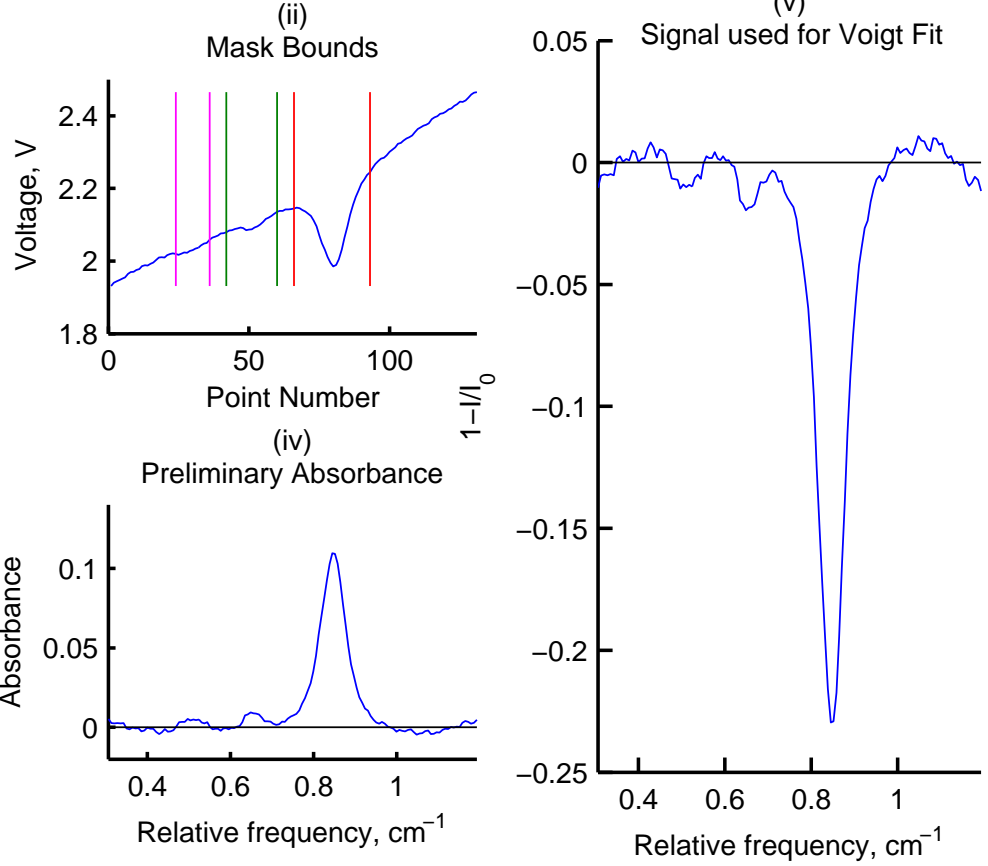

(b) 
(i)

Original Signal

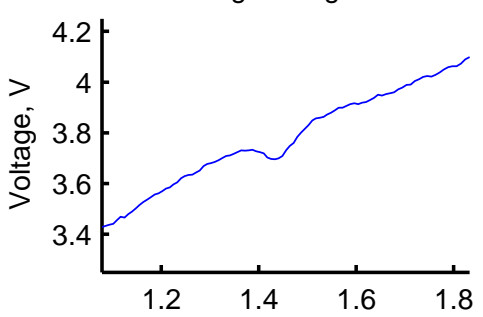

Relative frequency, $\mathrm{cm}^{-1}$

(iii)

Original Signal and Baseline Fit

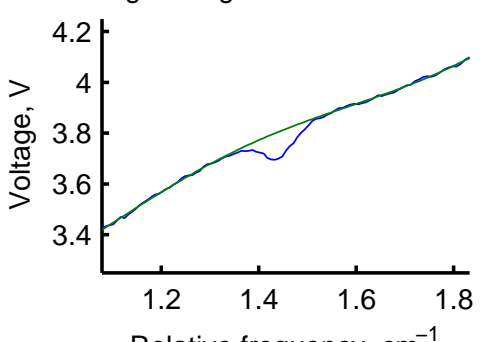

Relative frequency, $\mathrm{cm}^{-1}$

(i)

Original Signal

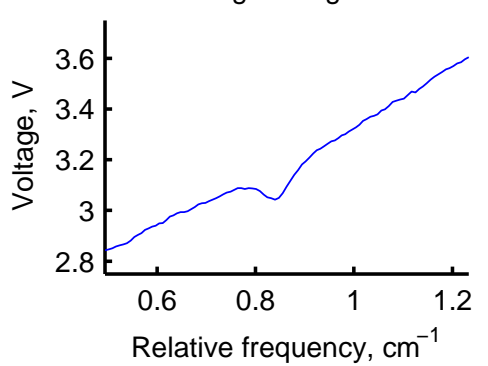

(iii)

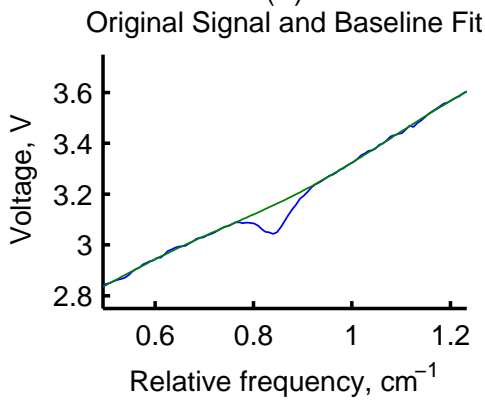

(ii)

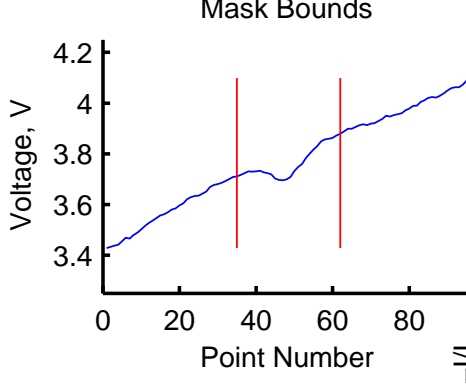

(iv)

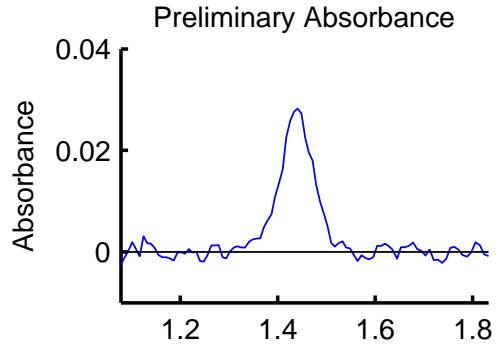

Relative frequency, $\mathrm{cm}^{-1}$

(c)

(ii)

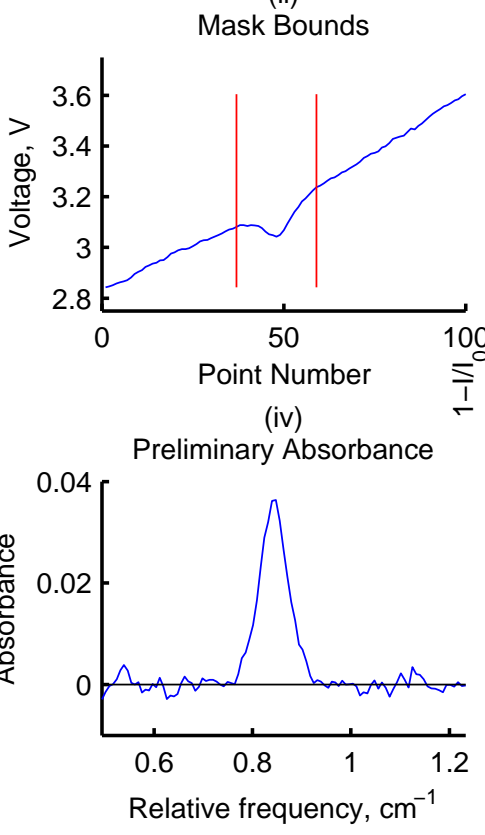

(d)

c) (v)

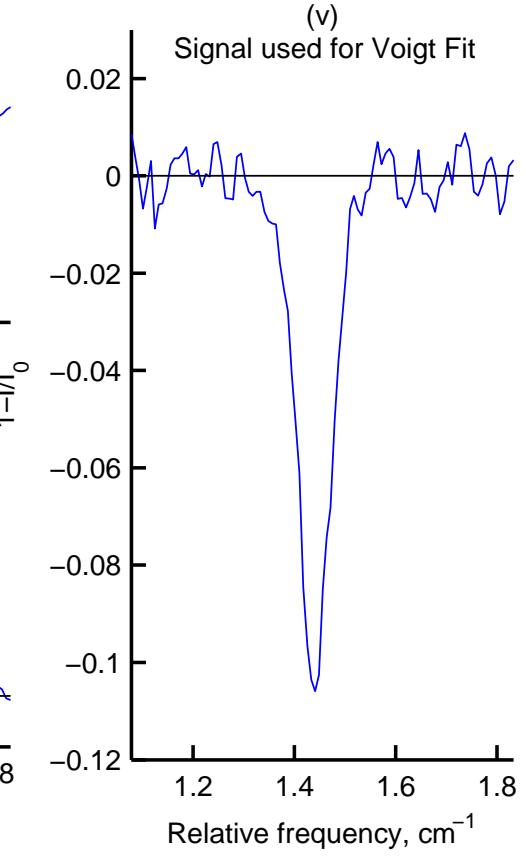

Relative frequency, $\mathrm{cm}^{-1}$ 
The Voigt profile can be approximated using several different models. Early work with the TDLAT technique utilized the modified Whiting approximation [52], given in Equation 2.17.

$$
\frac{I_{\lambda}}{I_{\lambda_{C L}}}=\left(1-\frac{w_{L}}{w_{V}}\right) \exp \left[-2.772\left(\frac{\lambda-\lambda_{C L}}{w_{V}}\right)^{2}\right]+\left(\frac{w_{L}}{w_{V}}\right) \frac{1}{1+4\left(\lambda-\lambda_{C L} / w_{V}\right)^{2}}
$$

where $I_{\lambda}$ is the wavelength-dependent intensity, $I_{\lambda_{C L}}$ is the wavelength-dependent intensity at line center, $w_{L}$ is the Lorentzian full width at half-maximum, $w_{V}$ is the Voigt full width at half-maximum, and $\lambda_{C L}$ is the line center wavelength, as seen in Figure 2.16.

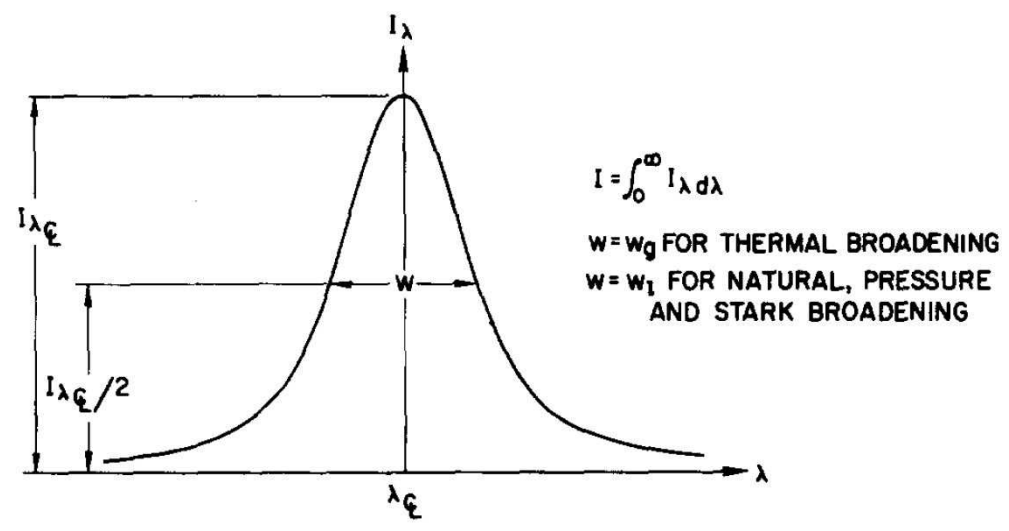

FiguRE 2.16: Diagram of Whiting approximation of Voigt profile. [52]

Due to long computation times, especially when multi-peak fits were utilized, and the implementation's tendency to wander to local, but not global, minima of the fitting parameter, a second Voigt approximation was used as described below.

The Voigt function is related to the complex error function, $W(z)$, as defined by the following integrals shown in Equation 2.18: 


$$
\begin{aligned}
W(z) & =\frac{i}{\pi} \int_{-\infty}^{\infty} \frac{\exp \left(-t^{2}\right)}{z-t} d t \\
W(z) & =K(x, y)+i L(x, y) \\
z & =x+i y \\
K(x, y) & =\frac{y}{\pi} \int_{-\infty}^{\infty} \frac{\exp \left(-t^{2}\right)}{y^{2}+(x-t)^{2}} d t \\
L(x, y) & =\frac{1}{\pi} \int_{-\infty}^{\infty} \frac{(x-t) \exp \left(-t^{2}\right)}{y^{2}+(x-t)^{2}} d t
\end{aligned}
$$

where $W(z)$ is the complex error function and $K(x, y)$ and $L(x, y)$ are the real and imaginary parts of $W(z)$ combined through superposition, respectively. [53] $W(z)$ can also be expressed using the complimentary error function er $f c$ of a complex argument, as $W(z)=\exp \left(-z^{2}\right) \operatorname{erfc}(-i z)$ with $\operatorname{erfc}(z)=1-\operatorname{erf}(z)$, where $z$ is defined by:

$$
\begin{aligned}
& z=x+y i \\
& x=\sqrt{\ln 2}\left(\nu-\nu_{0}\right) / \alpha_{D} \\
& y=\sqrt{\ln 2} \alpha_{L} / \alpha_{D}
\end{aligned}
$$

where $\alpha_{D}$ and $\alpha_{L}$ are the Doppler and Lorentzian widths, respectively, from Equation 2.3. When the terms $x$ and $y$ from Equation 2.19 are substituted into Equation 2.18d, it is seen that $K(x, y)$ is equivalent to the Voigt function seen in Equation 2.3. Thus, the Voigt function is the real portion of the complex complimentary error function. $[54]$

An efficient algorithm by Abrarov and Quine [53] based on exponential series approximation of the complimentary complex error function is shown in Equation 2.20 . 


$$
\begin{aligned}
w(z) & =\frac{i}{2 \sqrt{\pi}}\left[\sum_{n=0}^{N} a_{n} \tau_{m}\left(\frac{1-e^{i\left(n \pi+\tau_{m} z\right)}}{n \pi+\tau_{m} z}-\frac{1-e^{i\left(-n \pi+\tau_{m} z\right)}}{n \pi-\tau_{m} z}\right)-a_{0} \frac{1-e^{i \tau_{m} z}}{z}\right] \\
a_{n} & =\frac{2 \sqrt{\pi}}{\tau_{m}} \exp \left(-\frac{n^{2} \pi^{2}}{\tau_{m}^{2}}\right) \\
\tau_{m} & =10
\end{aligned}
$$

where $x, y$, and $z$ are input parameters and functions of the frequency, $\nu$, the linecenter frequency, $\nu_{0}$, and the Doppler and Lorentzian half-widths at half-maximum, $\alpha_{D}$ and $\alpha_{L}$ respectively. $w(z)$ is an approximation of the complex complimentary error function. Abrarov and Quine found in their work that good accuracy of the approximation to the exact value was obtained by setting $\tau_{m}$ to 10 and $N$ to 32 . [53]

Based on Abrarov and Quine's work, the following expression was used to make Voigt fits of the measured absorption profiles. The polynomial $b(z)$ was introduced to account for residual baseline contribution to the wings of the profile.

$$
\begin{aligned}
V(z) & =A m p \frac{1}{2 \sqrt{\pi}} \Im[w(z)]+b(z) \\
b(z) & =b_{0}+b_{1} z+b_{2} z^{2} \\
E & =\sum(V(z)-\text { signal })^{2}
\end{aligned}
$$

where $V(z)$ is the Voigt approximation summed with a secondary polynomial baseline, $b(z)$, and Amp is a scaling constant. The fitting routine utilizes MATLAB's fminsearchbnd to minimize the sum of squared errors $E$ between the Voigt approximation and the measured data. The user inputs initial guesses and lower and upper bounds on the following fit parameters: Amp, $\nu_{0}, \alpha_{L}, \alpha_{D}, b_{0}, b_{1}$, and $b_{2}$. For multi- 
peak Voigt fitting, as is performed for the KB1 and KB2 absorption features, there are Amp, $\nu_{0}, \alpha_{L}$, and $\alpha_{D}$ parameters for each absorption feature, with a condition set on the wavelength difference between $\nu_{0_{i}}$ and $\nu_{0_{i+1}}$, as is dictated by the physical separation of the peaks. Sample Voigt fits using the Abrarov approximation (Equations 2.19, 2.20, and 2.21) to each of the four absorption features are shown in Figure 2.17, with multi-peak fits utilized for KB1 and KB2 absorption features, and single-peak fits utilized for KB3 and KB4 absorption features.

Numerical integration is performed on each of the absorption features' Voigt profile fits. The vertical thick black lines in Figure 2.17 represent the boundaries past which the value of the Voigt profile is less than $2 \%$ of the maximum peak height. This $2 \%$ boundary is used as the limits of numerical integration. In the case that several absorption features are fit simultaneously, as in the case of KB1 and KB2, numerical integration is performed only for the main peak, i.e. the red profiles in Figures 2.17a and 2.17b. The resulting integrated absorbance values, obtained for each absorption feature for each LOS (each of the 1400 or 840 LOS) are the final product of Phase I of the analysis procedure. These integrated absorbance values are organized into four sinograms (one for each absorption feature), as is depicted in Section 2.3.1 and Figure 2.4. 


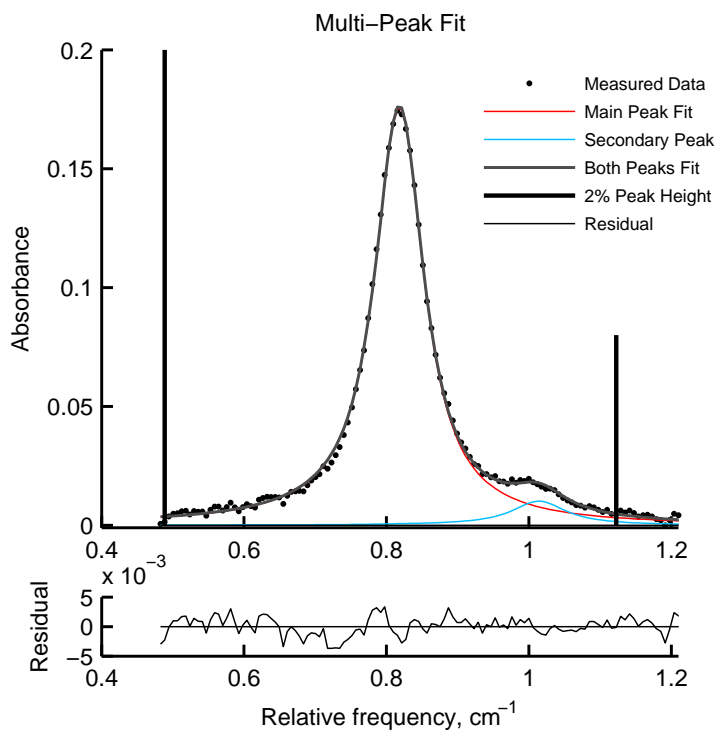

(a)

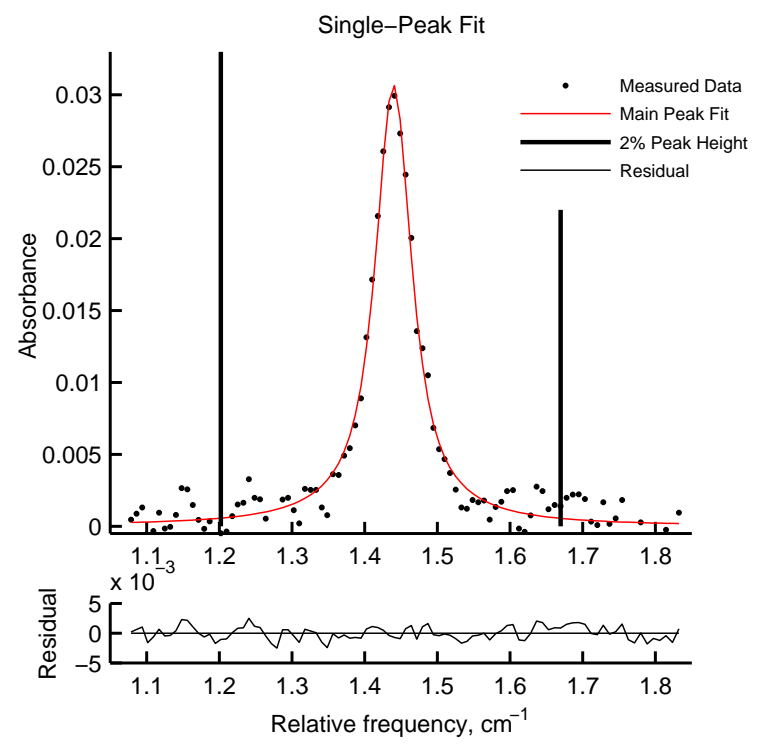

(c)

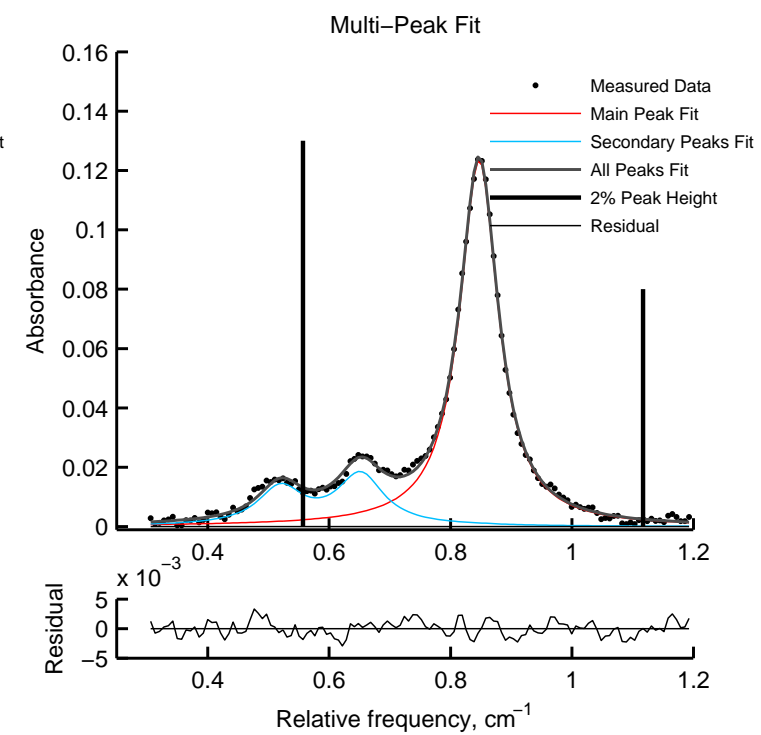

(b)

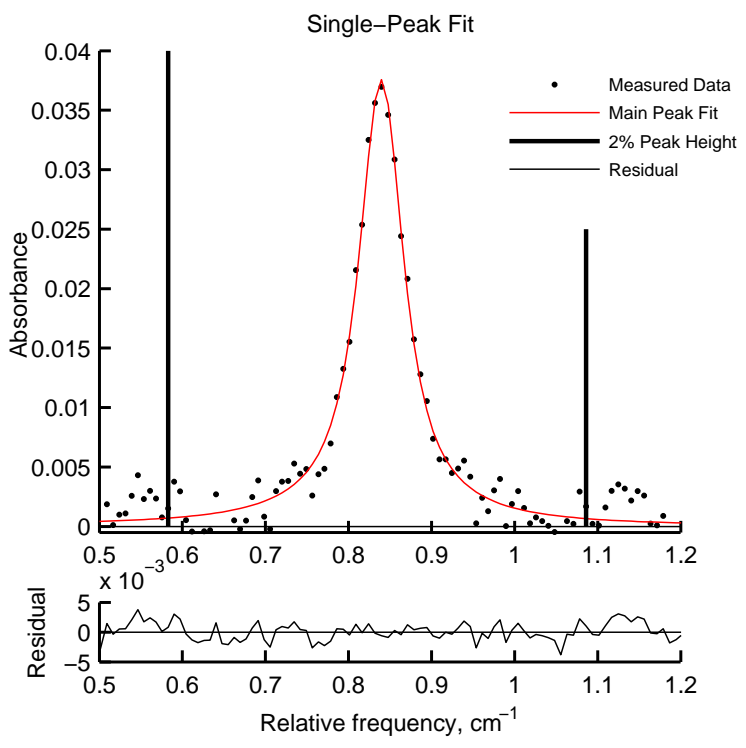

(d)

Figure 2.17: Sample multi-peak and single-peak Voigt fits for (a) KB1, (b) KB2, (c) KB3, and (d) KB4. 


\subsubsection{Phase II}

Tomographic inversion of the integrated absorbance sinograms is performed in Phase II. A median filter is applied to the measured sinograms to mitigate outliers. Though great care is taken in Phase I to identify "poor-quality" Voigt fits, automating the evaluation of the quality of fits for 4,200 spectra (9,800 when the multi-peak Voigt fits are included) for each data set has proven to be a major challenge of the TDLAT technique. Therefore, while the Voigt fit parameters and resulting integrated absorbances are monitored closely in Phase I, a median filter is utilized in Phase II to ensure outliers will not impact the tomographic reconstruction.

The ML-EM algorithm modeled after Verkruysse and Todd's [46] is utilized for tomographic inversion. Based initially on the "Future Work" of Bryner [31], the iterative ML-EM code allows for the user to specify the beam collection geometry (parallel-beam or fanbeam), the number and angles of the rays and fans, the physical size each pixel represents, and iteration tolerance. The output of Phase II analysis is four spatially-resolved distributions of integrated absorbance, one for each absorption feature. While no smoothing is performed during the ML-EM algorithm itself, a Gaussian filter is applied to the final reconstructed absorbance distributions. The Gaussian filter smooths in length-space, while also maintaining order in frequencyspace, and is coded to conserve total absorbance within $.01 \%$ of the original total absorbance.

For the experimental data considered, 200 iterations were found to be adequate for convergence of the ML-EM routine. As shown in Figure 2.18 for three sample pixels, the derivative of the pixel values is very small (less than .01\% of the pixel value) past 200 iterations. The ML-EM algorithm implementation has a tendency to impose superficial high frequency noise onto the reconstruction and therefore only 200 iterations are used. 


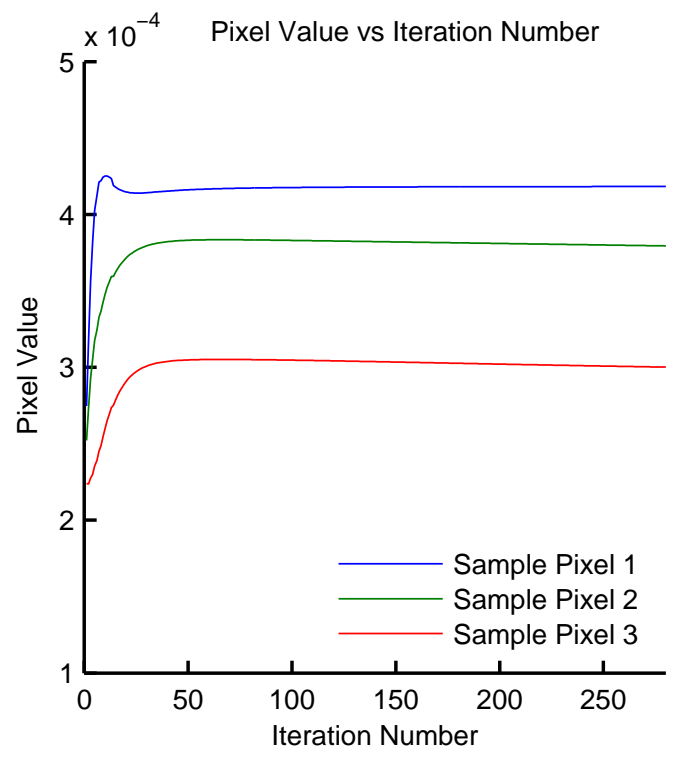

(a)

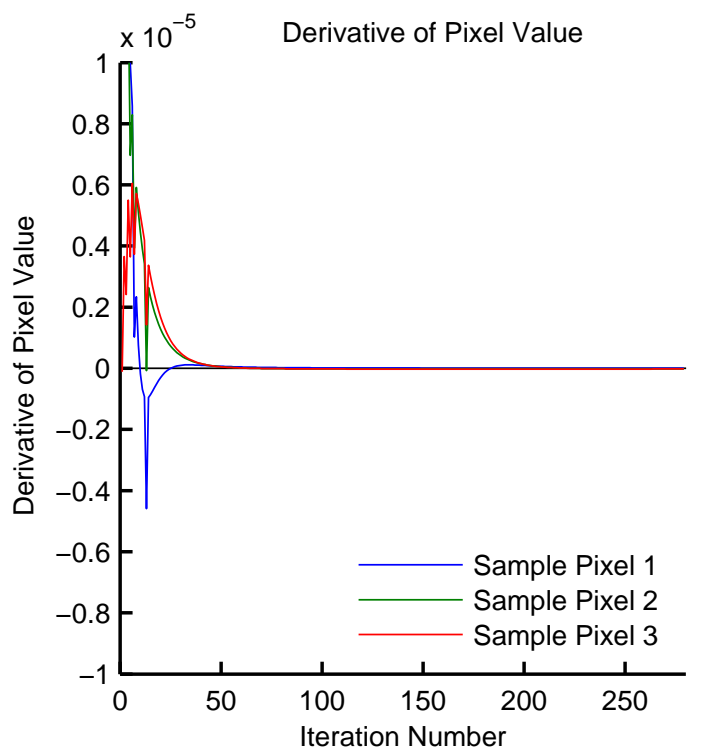

(b)

Figure 2.18: Convergence of ML-EM algorithm for sample pixels. (a) Pixel value versus iteration number for three sample pixels, and (b) the derivative of the pixel values with respect to the iteration number.

\subsubsection{Phase III}

Phase III consists of Boltzmann plot analysis or two-line thermometry (depending on the desired number of absorption features utilized) performed at each spatial pixel to obtain temperature and species number density values. At each spatial pixel, a non-linear least-squares fit is performed using the integrated absorbance values of each transition and their corresponding lower-state energies, as described in Section 2.2 .1 .

The KB4 absorption feature's spectral parameters were obtained from HITEMP 2010, but have not been independently measured and verified, as the other three absorption features have via Goldenstein et al. [48] Thus, this absorption feature will not be included in the forthcoming Boltzmann plot analysis due to the potential inaccuracy of its spectral parameters $S\left(T_{r e f}\right)$ and $E^{\prime \prime}$. As will be shown in Chapter 4, the inclusion of the KB4 absorption feature produces unrealistically high values of temperature and water vapor number density, leading to the decision to exclude 
this feature from Phase III analysis. Full analysis of the measured absorption of KB4 has been carried out in both Phases I and II, though, and future inclusion of the absorption feature in Phase III analysis is possible pending the availability of accurate spectral parameters. 


\section{Chapter 3}

\section{Tomographic Processing and Error Evaluation}

\subsection{Introduction and Motivation}

As was shown in Chapter 2, the TDLAT experiments discussed herein were collected in a fanbeam geometry, seen in Figure 2.3a. The infrared laser beams originate from vertices, $s_{i}$, and traverse the measurement region, which contains a distribution, $O$. The region of interest for this research is illustrated in Figure 3.1, which shows a cross-section of the exit plane of the scramjet model. Measurements will be collected at a plane slightly downstream of the actual UVaSCF exit plane. The large red rectangle is a cross-section of the UVaSCF hardware at the exit plane of the facility. Region A is defined by the internal geometry of the UVaSCF (the boundary of the scramjet flowpath) and was the principal region of interest. The scramjet exhaust will pass through Region A, and thus a majority of the high-temperature and high water vapor mole fraction gases should be contained within Region A, with some mixing between Region A and B expected. Region B (any area outside of Region A, including the area above the UVaSCF hardware itself) is represented by the cross-hatched area of Figure 3.1 and is considered to be of secondary importance. Region B is expected to 
have significantly lower temperature and lower water vapor mole fraction gases than Region A as it is outside of the "core flow" of the scramjet. Figure 3.1 shows that each ray of the example fanbeam pictured lies at least partially within Region B, as is true for all fanbeams measured in this experiment. The red rectangle in Figure 3.1 depicts the exit plane hardware geometry of the UVaSCF, but the same methodology can be extended to the NASA Langley DCSCTF.

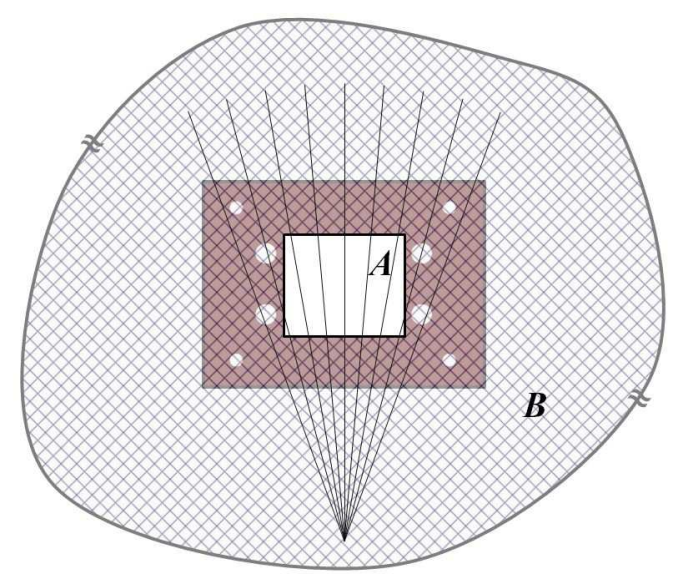

FIGURE 3.1: Schematic of region of interest.

This distinction between the expected gaseous properties of Regions A and B makes understanding the spectroscopic properties of the selected absorption features important. Four absorption features were utilized for the reported TDLAT experiments, one with a relatively low $E^{\prime \prime}, \mathrm{KB} 1$, one with a moderate $E^{\prime \prime}$, KB2, and two with relatively high $E^{\prime \prime}, \mathrm{KB} 3$ and KB4. Both the moderate- and high- $E^{\prime \prime}$ transitions will have absorption predominantly along the portions of their path lengths within Region A, with little-to-no detectable absorption along the portion of path lengths within Region B. This situation is analogous to an X-ray image, in which the radiation is absorbed by solid masses (bone, fat, muscle, etc.), but is transmitted through air, resulting in a "zero-background" image. The KB1 absorption feature, which has a relatively low lower-state energy, differs from the aforementioned absorption features because it has detectable absorption levels throughout both Regions A and B. This leads to an absorption distribution with a "non-zero background", which will 
be discussed at length in the following sections. This low lower-state energy level absorption feature, denoted as KB1, and its spectroscopic parameters were given in Table 2.2.

The TDLAT measurement system has been utilized for measurements of a model (sc)ramjet at the University of Virginia's Supersonic Combustion Facility (UVaSCF) for a non-fueled case as well as for two separate reacting fueling conditions, using four absorption features. Data analysis and tomographic reconstruction using the aforementioned ML-EM approach has manifested an artifact seen in the reconstruction of KB1, shown in Figure 3.2. Figure 3.2a shows the sinogram of measured KB1 absorbance and Figure 3.2b shows the subsequent reconstruction of KB1 absorbance. The black rectangle seen in Figure 3.2b denotes the geometry of the hardware exit, as was seen in Figure 3.1 called Region A. The artifact is seen to be low-valued streaks crossing the distribution at angles directly related to the sampling geometry. These streaks are generally known to be an aliasing error [38], and are due to an under-sampling of the measurement space. It is also seen in Figure 3.2b that as these low-valued streaks intersect, there is constructive interference, causing the value of the pixels at the intersection to be even further below their true value. It is evident that several of these low-valued streaks intersect in a circular ring and coalesce into a ring of very low-valued pixels at a radius of $33 \mathrm{~mm}$, as well as at $77 \mathrm{~mm}$.

Due to the physics of the experiment, it is highly unlikely that the integrated absorbance values seen in Figure $3.2 \mathrm{~b}$ would decrease as they pass from Region A to $\mathrm{B}$, then increase again in the far-field areas of Region B. It is much more likely that the values would decrease radially as approaching the boundary between Regions A and B, then plateau to a relatively constant value in Region B. Given the fixed number of samples collected, the manifestation of this aliasing error can be thought of as a result of attempting to reconstruct too large of a distribution grid. It would be desirable to simply reconstruct a smaller portion of this reconstruction grid, for example just inside the rectangular section of Region A. However, as discussed in the 


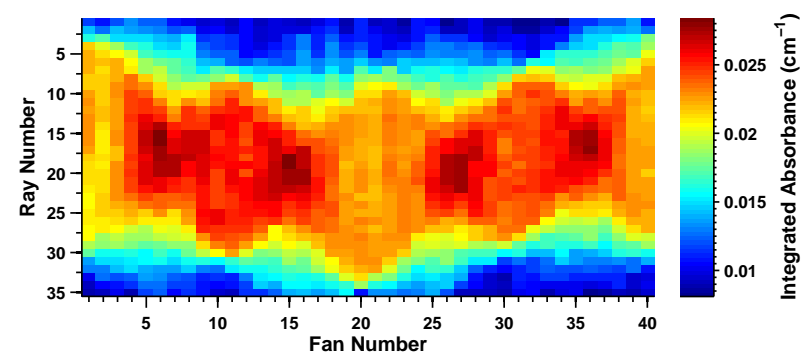

(a)

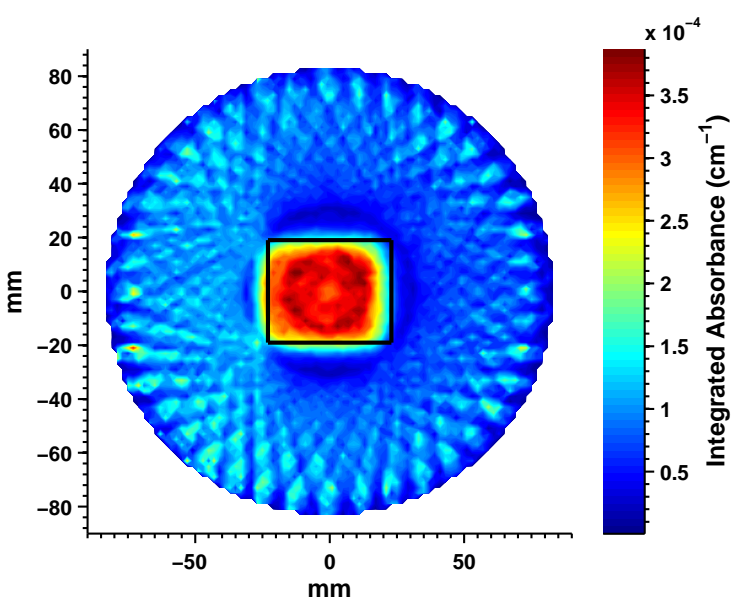

(b)

Figure 3.2: (a) Unaltered KB1 sinogram, and (b) unaltered KB1 reconstruction.

previous section, the KB1 absorption feature has detectable absorbance in Region $\mathrm{B}$, and thus part of the integrated absorbance values in a measured sinogram will contain signal originating in Region B. Therefore, if a smaller reconstruction grid is to be used, the background absorption contained within the measured sinogram originating from Region B must be accounted for in some way.

By summing the area matrix, $A_{i j}$ over each LOS, the amount of laser path length through each spatial pixel can be calculated. Figure 3.3a shows the distribution of total laser path length for each pixel in the reconstruction grid, by $\sum_{i} A_{i j}$ from Equation 2.14. Figure 3.3b shows the normalized radial gradient of this matrix, via Equation 3.1.

$$
F=\sum_{i} A_{i j}
$$

Normalized Radial Gradient of $F=\left[\left(\frac{\partial F}{\partial x}\right)^{2}+\left(\frac{\partial F}{\partial y}\right)^{2}\right]^{1 / 2} / \max (F)$ 


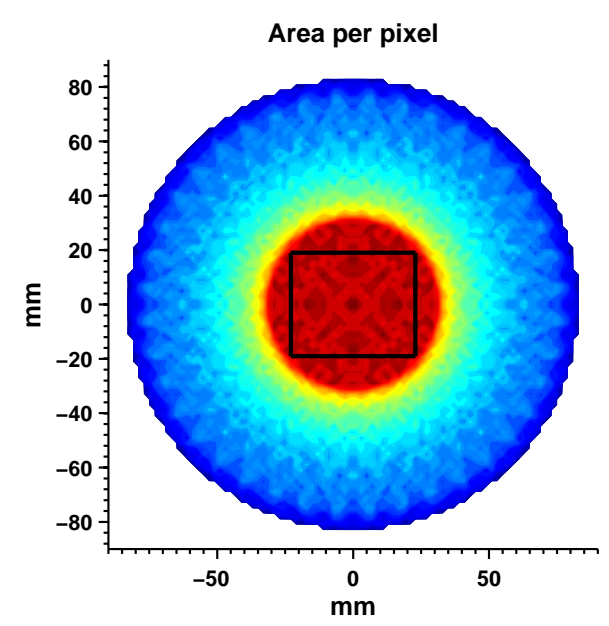

(a)

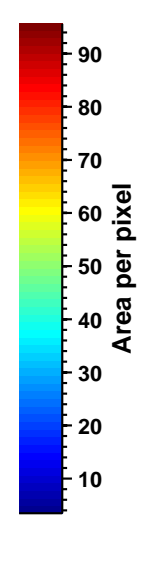

$$
\text { (1) }
$$

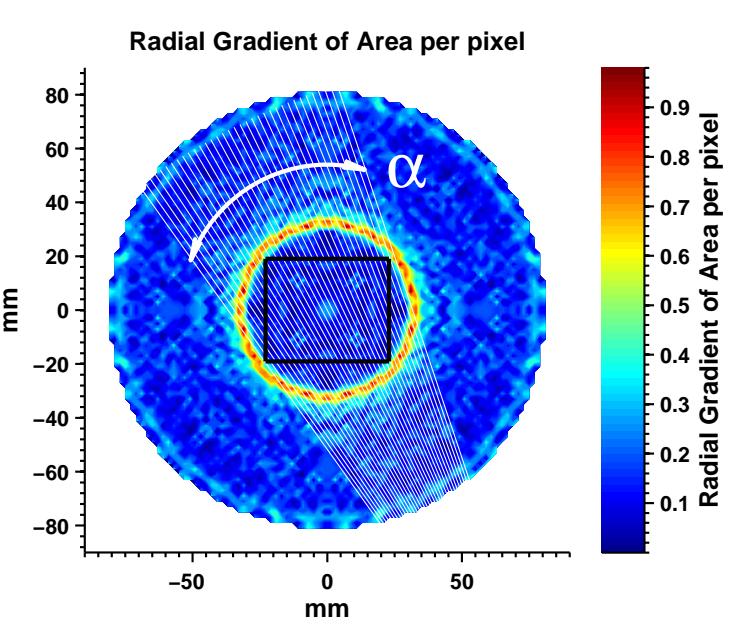

(b)

Figure 3.3: (a) Distribution of total laser path length per pixel, $\sum_{i} A_{i j}$, for experimental parameters shown in Table 2.1, and (b) normalized radial gradient of total laser path length per pixel, with a single fanbeam superimposed in white.

It is seen from Figure 3.3b that there is a large gradient in the amount of total laser path length per pixel at a radial distance of $33 \mathrm{~mm}$. This spike in radial gradient corresponds to the large drop-off in amount of total laser path length per pixel at this radius. This large decrease in amount of laser information attributed per pixel is due to the inscribed angle of the fanbeam, $\alpha$, as calculated by the perpendicular distance from the center of the distribution to the extreme rays. It is seen that the large radial gradient occurs in the pixel just beyond the reach of the most edge rays. The circular pattern of this large gradient is formed by the rotation of the 40 fanbeams to form the $360^{\circ}$ tomographic data set. The reason that this spike in radial gradient is inscribed by the black rectangle denoting Region $\mathrm{A}$ is because the total fan angle, $\alpha$, was chosen to completely capture Region A at its maximum width (along a diagonal), as is illustrated by Figure 3.3b. It can be shown that the radial location of the spike in radial gradient varies exactly with $\alpha$.

It is now discernible that this spike in radial gradient lies in the same location as the low-valued ring artifact seen in Figure 3.2b. Indeed, even a secondary spike in radial gradient at $77 \mathrm{~mm}$ radial distance is apparent in Figure $3.2 \mathrm{~b}$ as a low-valued 
ring artifact at the same radial distance. It is then desirable to determine what effect this low-valued ring artifact has on the accuracy of the reconstruction.

In attempt to better understand this artifact, a representative synthetic distribution was created. The reconstruction of an oblong smoothed top-hat distribution is shown in Figures 3.4a-3.4d, which has a far-field value 0 and a peak value of 7 . From the synthetic distribution, a sinogram is formed by multiplying the $A_{i j}$ matrix by the vector of reconstruction cells, described in Equation 2.15. This synthetically "measured" sinogram is then the input sinogram, $\hat{P}_{i}$, to the ML-EM algorithm. The ML-EM algorithm is executed just as it is with experimental data, and the final reconstructed distribution is shown in Figure 3.4b. The excellent agreement between Figure 3.4a and Figure 3.4b, is elucidated by a plot of the absolute difference between the two, Figure 3.4c. The sinogram of this distribution, normalized by its maximum value, is shown in Figure 3.4d. It is seen that the normalized sinogram of the zero-background distribution ranges from zero to one, with values of zero in the edge rays.

Figures $3.4 \mathrm{e}-3.4 \mathrm{~h}$ show the same reconstruction process, but instead a slightly altered synthetic distribution is considered. Shown in Figure 3.4e, the altered synthetic distribution ranges from 3-10, with a far-field value of 3 . This distribution, with a non-zero background, better captures the distribution of interest, KB1, because of its detectable absorption in Region B. The values of Figure 3.4e and Figure 3.4f have been windowed to a range of $2-6$, such that anything larger than 6 will be shown as pure white to emphasize differences. The absolute difference between Figure 3.4e and Figure 3.4f are displayed in Figure 3.4g. These differences are more numerous and larger in magnitude than those seen in the zero-background distribution, and resemble very closely the circular artifact observed in experimental data reconstructions, Figure $3.2 \mathrm{~b}$. The low-valued ring of error is seen surrounding the rectangular outline of Region A, as well as along the border of the circular reconstruction grid. While the distributions each have a difference of 7 between the far-field value and the peak 
value, it is notable that the normalized sinograms show a significant difference in dynamic range. The normalized sinogram of the zero-background distribution ranges from zero to one, while the normalized sinogram of the non-zero background distribution ranges only from 0.7 to 1.0. Thus, the existence of a non-zero background value appears to introduce significant imaging artifacts in this usage of the ML-EM algorithm.

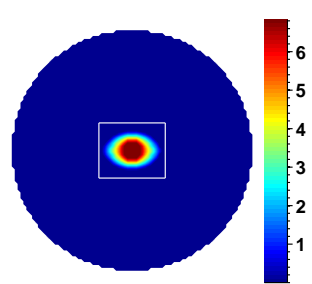

(a)

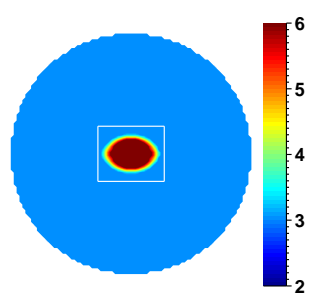

(e)

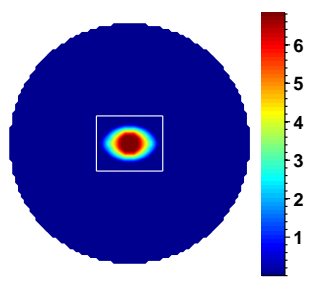

(b)

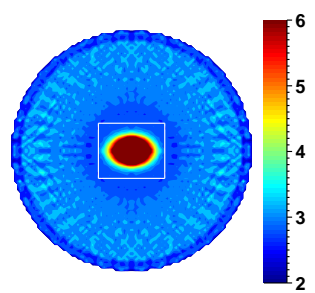

(f)

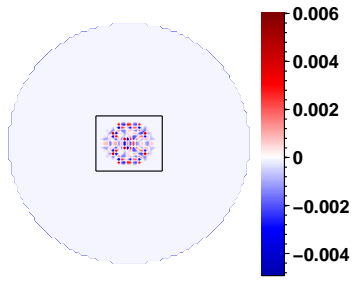

(c)

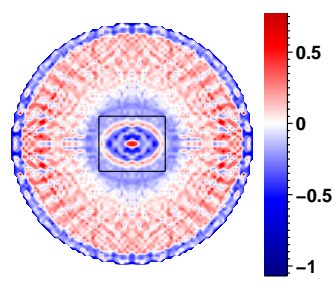

(g)

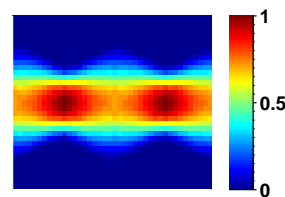

(d)

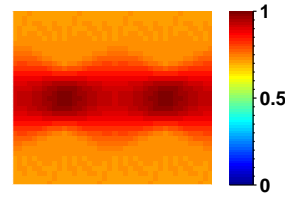

(h)

FiguRE 3.4: (a) Zero-background original distribution, (b) zero-background reconstruction, (c) zero-background error, (d) zero-background normalized sinogram, (e) non-zero background original distribution, (f) non-zero background reconstruction, (g) non-zero background error, and (h) nonzero background normalized sinogram.

It also bears mentioning that in Figures $3.4 \mathrm{~b}$ and $3.4 \mathrm{f}, 5,544$ grid cells are being reconstructed, due to a resolution of $2 \mathrm{~mm} \times 2 \mathrm{~mm}$ and the geometry given in Table 2.1. The reconstruction ratio, $R$, given as the ratio of the number of measurements to the number of grid cells, is 0.25 for 1,400 LOS and 5,544 grid cells. Generally, $R$ should be larger than or equal to 1 . $R \ll 1$ indicates that there is significant undersampling (observed in this research), while $R \gg 1$ indicates that a greater resolution may be achievable.

For the TDLAT experiments presented in this research, the spectroscopic proper- 
ties of the KB1 absorption feature result in an absorbance distribution with a nonnegligible, non-zero background. Therefore, it is critical that the far-field absorbance be managed. Though larger reconstruction cells could be utilized, it is also desirable that the reconstruction produce a distribution with a resolution near $2 \mathrm{~mm} \times 2 \mathrm{~mm}$ in the region of greatest interest.

Therefore, an approach is needed to account for the non-zero far-field absorbance, while also maintaining a grid resolution of $2 \mathrm{~mm} \times 2 \mathrm{~mm}$. Also, as is the case with any measurement, an estimation of uncertainty is desired for TDLAT results. While the uncertainty due to the absorption spectroscopy aspect of TDLAT is welldocumented [37], the uncertainty due to the ML-EM algorithm implementation is not well-defined for these measurements. A secondary motive for this analysis, then, is the quantification of the uncertainty of the TDLAT results with mitigated non-zero background values due to the ML-EM algorithm implementation.

\subsection{Method}

To better understand and manage the far-field absorbance, several 2-D distributions are created which are representative of the distribution of KB1 absorbance. Properties of the distribution that were considered were: dynamic range, type and level of noise, and far-field value. A method of background subtraction is proposed, which would serve to both mitigate the non-zero background distribution, as well as decrease the total number of grid cells reconstructed. This decrease in the total number of grid cells will eliminate the reconstruction grid cells far outside of the main region of interest, Region $\mathrm{A}$, while preserving a spatial resolution of $2 \mathrm{~mm} \times 2 \mathrm{~mm}$ per grid cell within Region $\mathrm{A}$. This will increase the value of $R$, mitigating the artifacts resulting from the under-sampled measurement geometry.

First, nine trial distributions were generated to each mimic certain properties of the KB1 absorbance distribution. Figures 3.5 and 3.6 show these nine distributions, 
for values of $0 \%$ and $30 \%$ noise addition respectively. Two other intermediate noiseaddition cases were also studied, for $10 \%$ and $20 \%$ noise additions, but are not pictured for brevity. The type of noise addition is varied between the distributions, as listed in Table 3.1. A background noise addition is determined such that only the pixels which are at the background value will have noise added to them. Pseudorandom noise is added to the background pixels according to the following equation:

$$
D_{\text {new }}(x, y)=D_{\text {original }}(x, y)+p \cdot B \cdot r(x, y)
$$

where $D_{\text {original }}(x, y)$ and $D_{\text {new }}(x, y)$ are the pixel values before and after noise addition, respectively, $p$ is a multiplier which scales the intensity of the noise and takes on values of $0.0,0.10,0.20$, and 0.30 , corresponding to the levels of noise addition, $B$ is the value of the background pixels, which is constant for all distributions and is the far-field value, and $r(x, y)$ is a matrix of pseudorandom numbers which are obtained from a standard normal distribution. A constant noise addition means that all pixels will have noise addition. The parameters for Equation 3.2 are the same as for the background noise case, but in this case $D_{\text {new }}$ and $D_{\text {original }}$ include all pixels in the distribution. This case of noise addition simulates electronic noise. An inversely proportional noise addition is such that the pixel will have a noise addition inversely proportional to its value, simulating scan-to-scan noise. This type of noise is prescribed normalized to the maximum value of the distribution, and takes the same general form as Equation 3.2 except $B$ is now a 2-D distribution of the form $B(x, y)=\max \left(D_{\text {original }}\right) / D_{\text {original }}$. Adhering strictly to Equation 3.2 introduces the possibility of having negative pixel values after noise addition. In keeping true to the physical process of absorption, a negative pixel value (representing absorbance) is irrational. Therefore, these few occurrences were mitigated by multiplying the negative pixel values by -0.5 , producing a positive, but very small pixel value. 


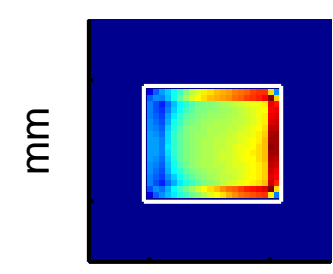

$\mathrm{mm}$

(a)

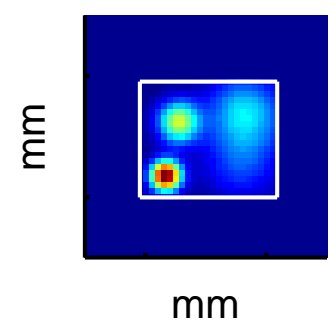

(d)

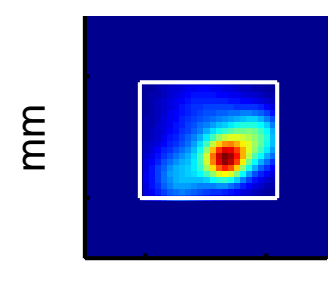

$\mathrm{mm}$

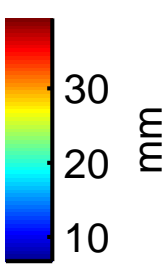

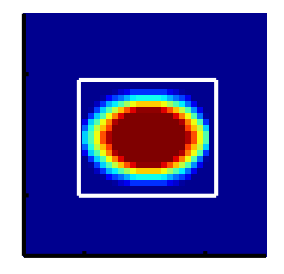

$\mathrm{mm}$

(b)

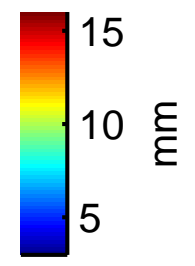

$$
\text { ( }
$$

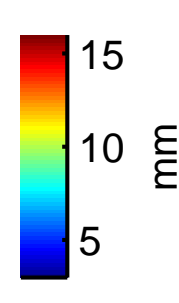

(g)

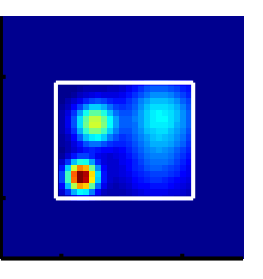

$\mathrm{mm}$
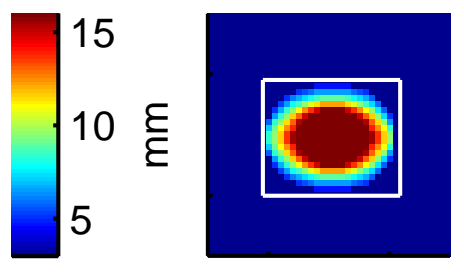

$\mathrm{mm}$

(c)

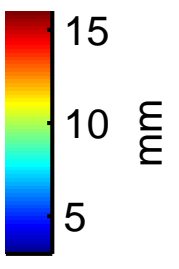

e)

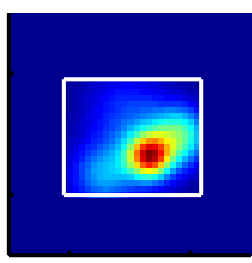

$\mathrm{mm}$

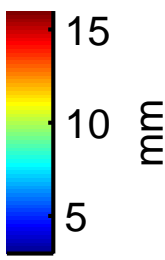

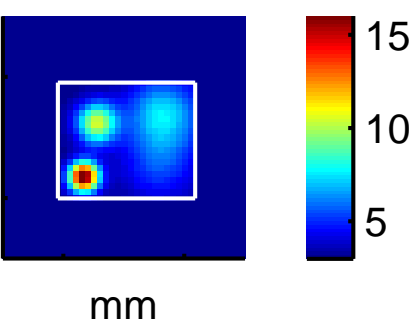

(f)

(h)
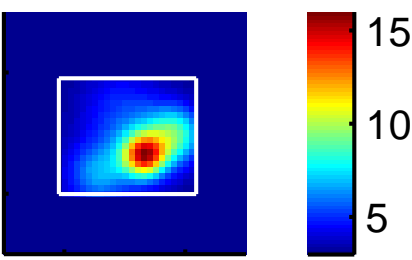

$\mathrm{mm}$

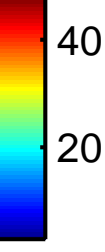

20 


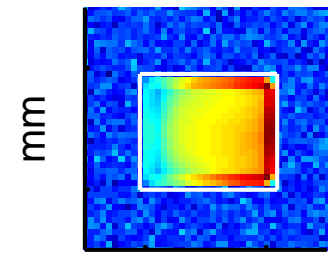

$\mathrm{mm}$

(a)

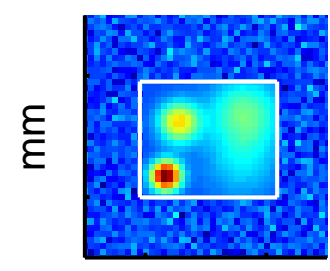

$\mathrm{mm}$

(d)

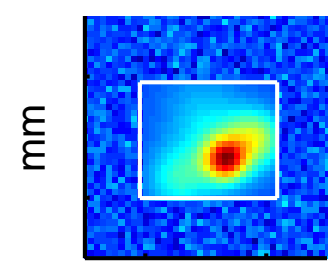

$\mathrm{mm}$
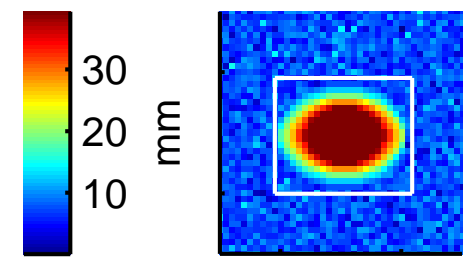

$\mathrm{mm}$

(b)
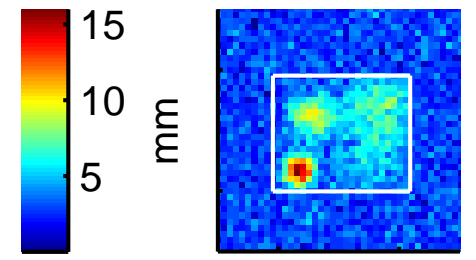

$\mathrm{mm}$

(e)

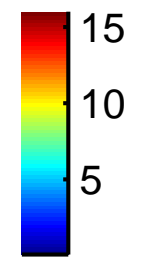

(g)

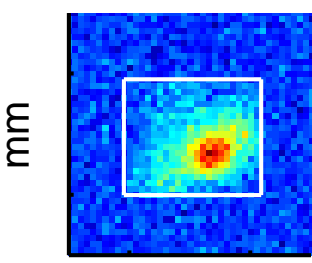

$\mathrm{mm}$

(h)
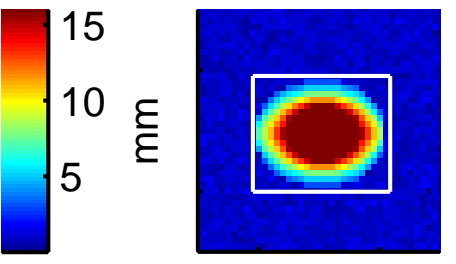

$\mathrm{mm}$

(c)
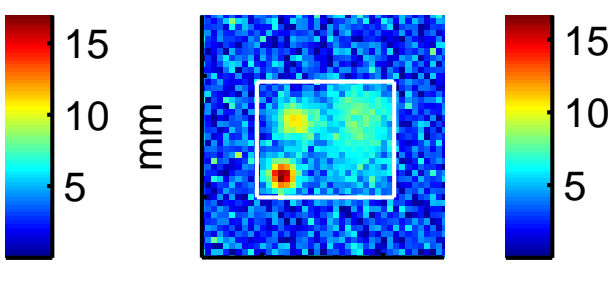

$\mathrm{mm}$

(f)
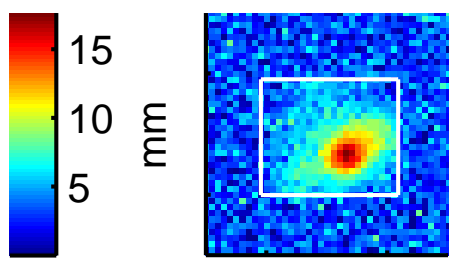

$\mathrm{mm}$

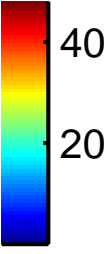

20

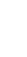


Distribution 1 of the nine trial distributions is the predicted distribution for the KB1 absorbance distribution created from the temperature and water vapor distributions via a computational fluid dynamics (CFD) study performed by Edwards and Fulton at N.C. State University [55]. Its far-field value, as well as dynamic range are expected to be reasonable approximations for the actual experimental distribution, and therefore is an excellent computational surrogate distribution. It is observed that there are very steep gradients existing along the four cardinal boundaries of this distribution. Noise is only added to the background-value pixels of this distribution and the percentage of noise indicates an addition of random noise which has a maximum of $30 \%$ of the far-field value, for example. Distributions 2 and 3 are identical to each other, except that the internal pixels (non-background pixels) are scaled by a value of three. This scaling allows for two distributions to be directly compared to each other, with the same shape but extremely different dynamic ranges, 5.33 and 16 respectively.

TABLE 3.1: Summary of properties of the nine synthetic distributions created for study, as shown in Figures 3.5 and 3.6.

\begin{tabular}{llllll}
\hline Distribution & Figure & Far-Field Value & Maximum & Dynamic Range & Noise Type \\
\hline 1 & $3.5 \mathrm{a}, 3.6 \mathrm{a}$ & $6.77 \times 10^{-5}$ & $3.94 \times 10^{-4}$ & 5.82 & background only \\
2 & $3.5 \mathrm{~b}, 3.6 \mathrm{~b}$ & 3.0 & 16 & 5.33 & background only \\
3 & $3.5 \mathrm{c}, 3.6 \mathrm{c}$ & 3.0 & 48 & 16 & background only \\
4 & $3.5 \mathrm{~d}, 3.6 \mathrm{~d}$ & 3.0 & 16 & 5.33 & background only \\
5 & $3.5 \mathrm{e}, 3.6 \mathrm{e}$ & 3.0 & 16 & 5.33 & constant \\
6 & $3.5 \mathrm{f}, 3.6 \mathrm{f}$ & 3.0 & 16 & 5.33 & inversely proportional \\
7 & $3.5 \mathrm{~g}, 3.6 \mathrm{~g}$ & 3.0 & 16 & 5.33 & background only \\
8 & $3.5 \mathrm{~h}, 3.6 \mathrm{~h}$ & 3.0 & 16 & 5.33 & constant \\
9 & $3.5 \mathrm{i}, 3.6 \mathrm{i}$ & 3.0 & 16 & 5.33 & inversely proportional \\
\hline
\end{tabular}

Distributions 4, 5, and 6 are all generated from the same distribution, but have varying types of noise added to them, as given in Table 3.1. This distribution is comprised of five Gaussian peaks, with randomized center locations and strengths, 
via the equations:

$$
\begin{aligned}
P_{i}(x, y) & =\frac{a}{2 \pi s_{i}^{2}} \exp \left(-\frac{\left(x-\mu_{x_{i}}\right)^{2}+\left(y-\mu_{y_{i}}\right)^{2}}{2 s_{i}^{2}}\right) \\
P_{\text {total }}(x, y) & =\sum_{i=1}^{5} P_{i}(x, y)
\end{aligned}
$$

For distributions 4,5 , and 6 , each $s_{i}, \mu_{x_{i}}$, and $\mu_{y_{i}}$ were randomly selected once, and then kept constant during the noise addition process. Similarly, distributions 7, 8, and 9 were created in the same manner, but with different values of $s_{i}, \mu_{x_{i}}$, and $\mu_{y_{i}}$. Any values past the rectangular boundary of Region A were truncated to be the value of the far-field. Distributions 4 through 9 were generated with the goal of studying distributions with a very low level of symmetry present.

TABLE 3.2: Summary of randomly generated parameters used in Equations 3.3a and 3.3b to create six of the nine synthetic distributions, as shown in Figures 3.5d-3.5i and Figures 3.6d-3.6i.

\begin{tabular}{llll}
\hline Distribution & $S_{i}$ & $\mu_{x_{i}}$ & $\mu_{y_{i}}$ \\
\hline \multirow{4}{*}{$4,5,6$} & 4.7533 & -10.102 & -5.7389 \\
& 7.7749 & 9.8554 & -9.4223 \\
& 3.5217 & -14.39 & 11.925 \\
& 9.5812 & 15.546 & -7.8154 \\
& 8.7316 & 10.131 & 8.1014 \\
\hline \multirow{4}{*}{8,8} & 10.175 & -2.5984 & -9.1635 \\
& 6.3364 & 15.755 & -0.86395 \\
& 7.5268 & -10.143 & 12.446 \\
& 8.4285 & 6.0401 & 4.3431 \\
& 4.6528 & 5.1276 & 6.1685 \\
\hline
\end{tabular}

To remove the non-zero background from any of these distributions would be very simple, as they were created computationally and the background value is known. For the experimental measurements, however, the far-field background value is not known, and thus a DC component of the reconstructed distribution cannot simply be subtracted from the distribution itself, but rather will need to be integrated into the measured sinogram. A priori, the level of background absorbance that needs to be 
subtracted is unknown. Therefore, a methodology was sought which can be used to determine the proper level of background subtraction needed.

To seek the method, several annuli of constant value with prescribed inner diameter (ID) and value are constructed. The value of the annulus will be some percentage of the actual background value of the distributions of interest, either $6.77 \times 10^{-5}$ for distribution 1 or 3.0 for distributions 2-9. The percentages considered are $60 \%, 75 \%$, $90 \%, 100 \%, 110 \%, 125 \%$, and $140 \%$ of the nominal background value. The outer radius of the annulus is fixed at $84 \mathrm{~mm}$, which is the maximum radius from the center of the measurement region that the rays will reach. The inner radius of the annulus will be varied from $25 \mathrm{~mm}$ to $50 \mathrm{~mm}$. The resulting annulus is then synthetically measured to form a sinogram, by multiplying the annulus by the area matrix $A_{i j}$.

This annulus sinogram is then subtracted from the synthetic sinogram formed from each of the nine distributions. The resulting sinogram is then reconstructed over the circular reconstruction grid bounded by the inner radius of the annulus. Each reconstruction is performed using the ML-EM algorithm described above. A subset of the reconstructed values (those which fall within Region A) are saved for further analysis.

A matrix is therefore formed which has dimensions $480 \times 7 \times 26 \times 9 \times 4$, which corresponds to the number of grid points, the number of \% background value annulus subtraction, the number of annuli ID, the number of distributions, and the number of levels of noise addition values, respectively. It is postulated that the reconstructions with the "correct" background value subtracted, i.e. $100 \%$ of the nominal value, will have the least amount of variation in pixel value as the ID of the annulus is varied. If this is shown to be the case with the simulated distributions, this process could be repeated with real experimental measurements. Potentially, a range of background subtraction values could be tested, with the "correct" value identified as the case which shows minimum pixel variation over the range of annulus ID.

For each of the nine distributions and for all four levels of noise addition, the final 
reconstructed value of each individual pixel within the region of interest is tracked as it varies, depending on the total grid radius. Figure 3.7 shows how two example pixels vary over grid radius. The example shown is for distribution 2 with $0 \%$ noise addition.

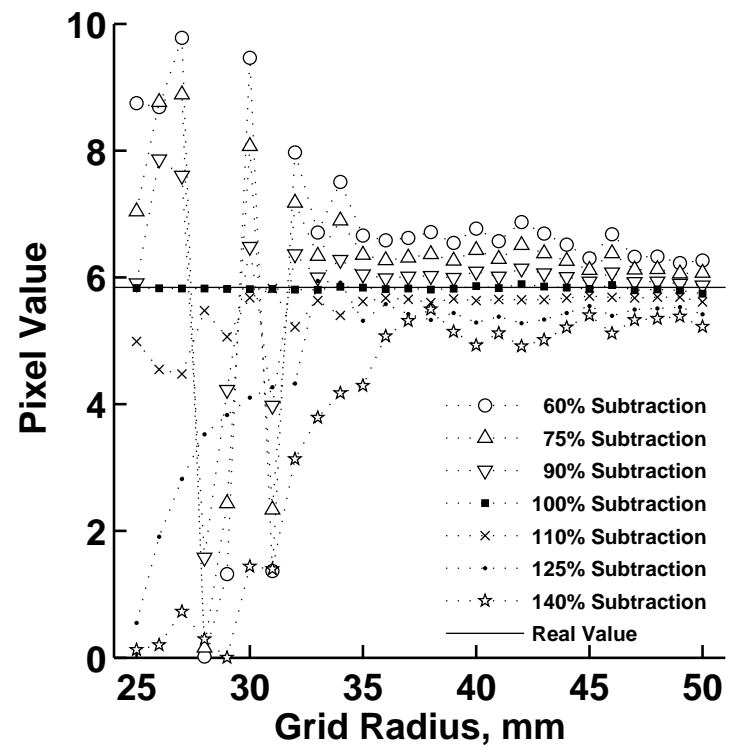

(a)

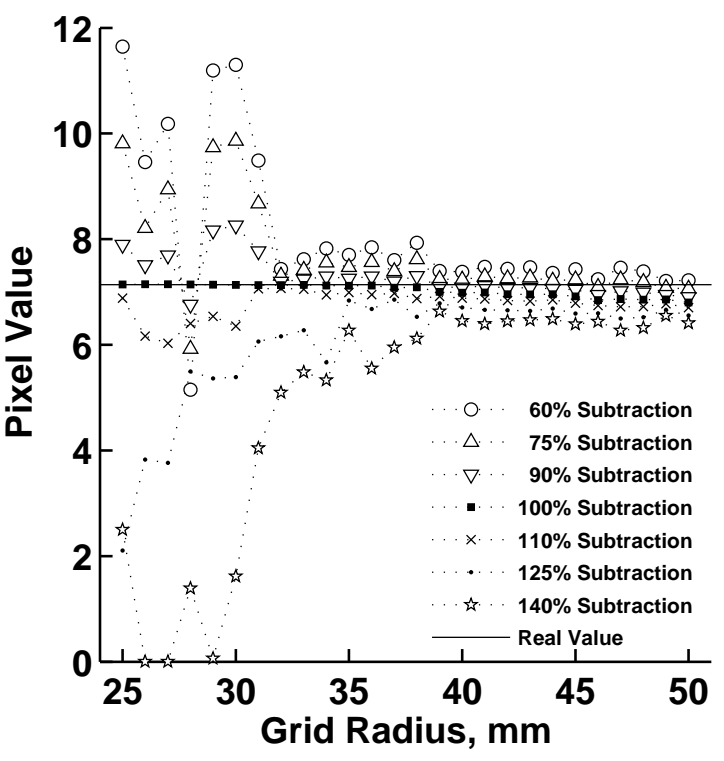

(b)

Figure 3.7: (a) The evolution of a first example point for varied levels of background subtraction across varying grid radius, and (b) the evolution of a second example point for varied levels of background subtraction across varying grid radius.

To consolidate this information, the standard deviation of each background subtraction case is calculated over the range of grid radii. This pixel-by-pixel variation is then quantified for all 480 pixels within the region of interest, for each background subtraction case, for each distribution, and for each case of noise addition. Figure 3.8 shows the pixel-by-pixel variation for the first 60 points of distribution 2 with $0 \%$ noise addition. 


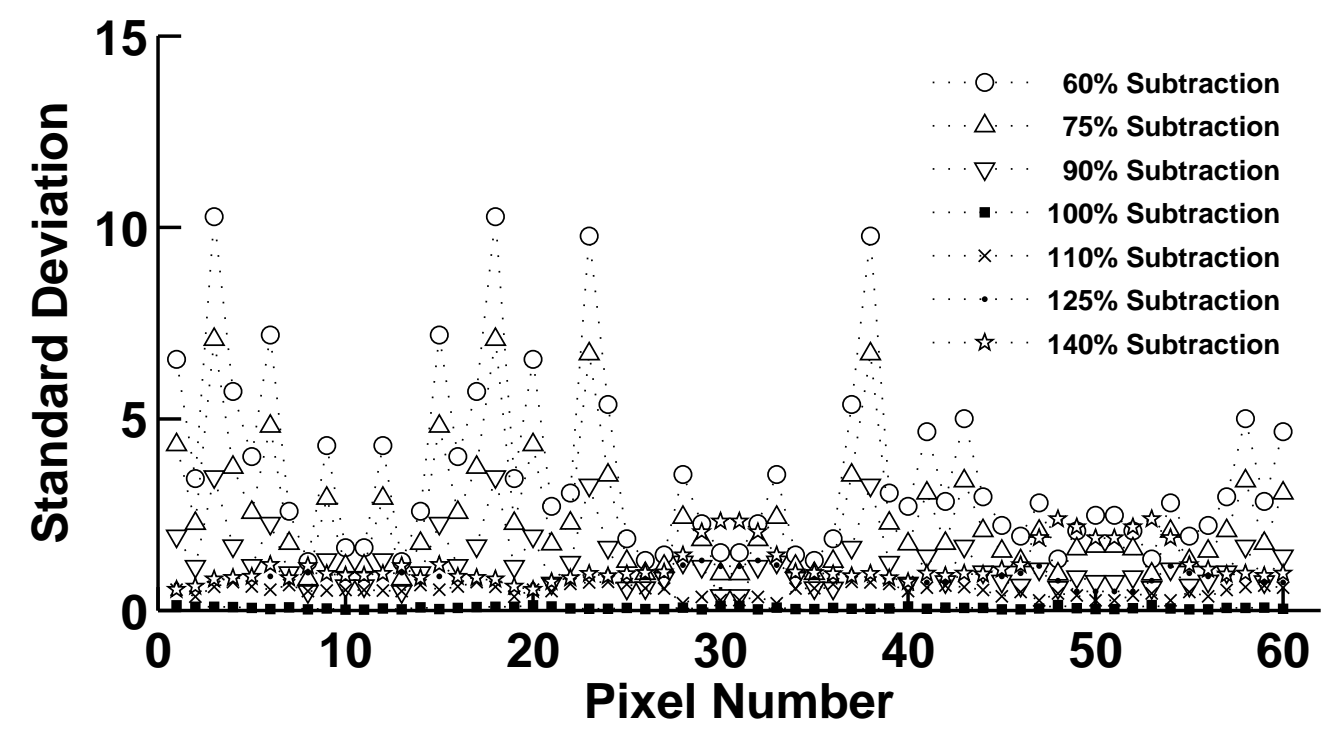

FiguRE 3.8: The standard deviation for the first sixty points of distribution 2 with $0 \%$ noise addition for all seven background subtraction values.

To consolidate further, the mean and standard deviation of each subtraction case is calculated over all 480 points within the region of interest. The resulting information is now the mean and standard deviation of pixel variation (the standard deviation of pixel values varying with annuli diameter) for each of the nine distributions, the four noise addition cases, and seven subtraction cases. A summary of this information for distribution 2 is shown in Figure 3.9.

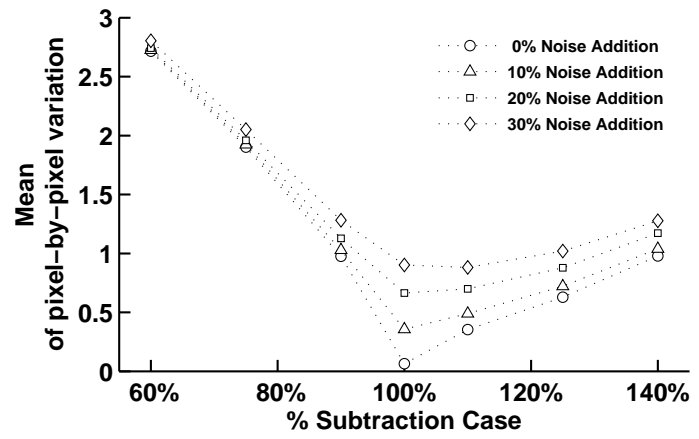

(a)

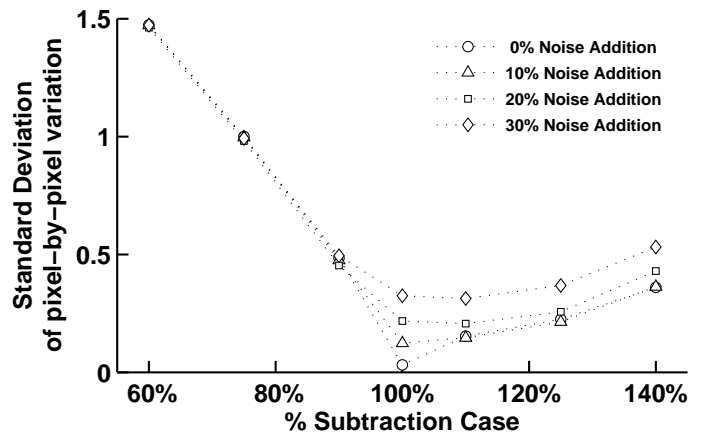

(b)

FiguRE 3.9: (a) The mean of pixel-by-pixel variations for distribution 2 for all four noise addition cases versus the \% subtraction case, and (b) the standard deviation of pixel-by-pixel variations for distribution 2 for all four noise addition cases versus the $\%$ subtraction case.

The mean of pixel-by-pixel variation will be utilized in this study, but the stan- 
dard deviation plot shown in Figure $3.9 \mathrm{~b}$ demonstrates that the standard deviations indeed follow the same trends as the mean of pixel-by-pixel variations do. It is seen that with increasing noise addition, the overall mean pixel-by-pixel variation for each distribution will increase, due to the intrinsically noisy nature of these distributions. Figure 3.10 shows the mean pixel-by-pixel variation plots for each of the nine trial distributions for all four noise addition cases.

It is remarkable how similar the plots in Figure 3.10 are to each other. As was predicted, generally the minimum for each distribution is the $100 \%$ subtraction case. The asymmetry in the pixel-by-pixel variation plots across the $100 \%$ plane is seen in each of the distributions and for all noise cases. This asymmetry indicates that under-subtracting the background value from a distribution results in a much more erratic distribution with respect to grid-to-grid fluctuation. The under-subtraction has a much sharper edge than the over-subtraction cases do. This suggests that the preferred method of finding the minimum point of the mean pixel-by-pixel variation plot is starting with low subtraction values, and increasing this subtracted value until the mean pixel-by-pixel variation begins to increase. If approached from the over-subtraction side, the slope is less steep and may not produce the desired result. It is also evident that with increasing noise addition, the slopes of both undersubtraction and over-subtraction flatten, obscuring the minimum further especially when approached from the right-hand side.

To quantify this finding, the global minimum of each noise addition case for each distribution is obtained based purely on the calculated pixel-by-pixel means. This results in four minima per distribution: one for each noise addition case. The mean and standard deviation of each of these sets is calculated, resulting in one point for each distribution. This summary of pixel-by-pixel variation is shown in Figure 3.11. The means, along with error bars representing \pm the standard deviations, indicate that using this method to identify the "correct" subtraction value (i.e. 100\%) results in a subtraction value that is within $11 \%$ of the actual value. This summary incorpo- 


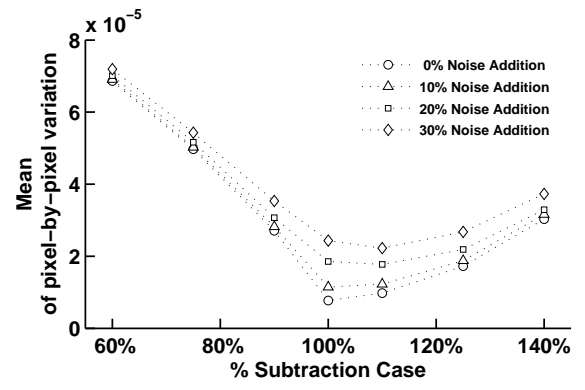

(a)

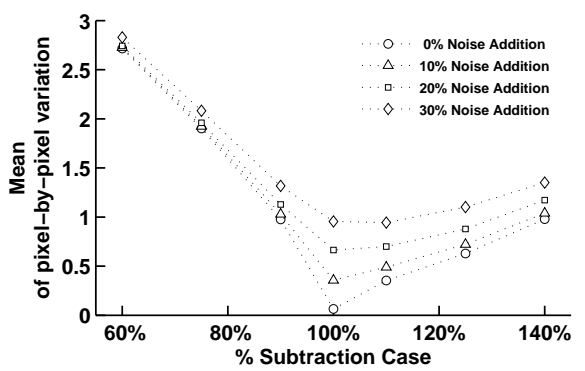

(c)

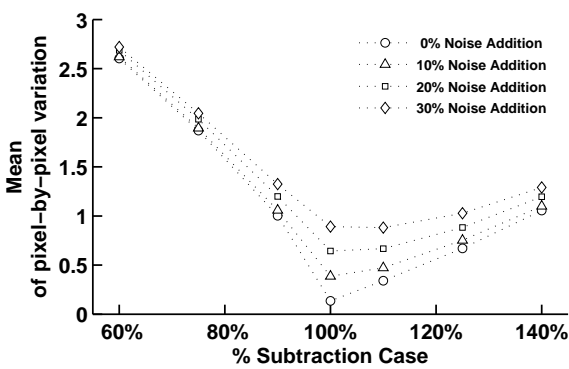

(e)

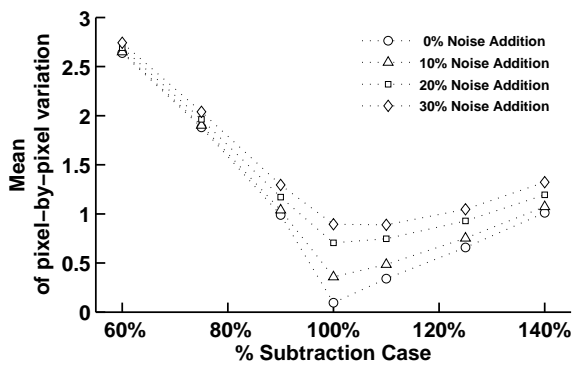

(g)

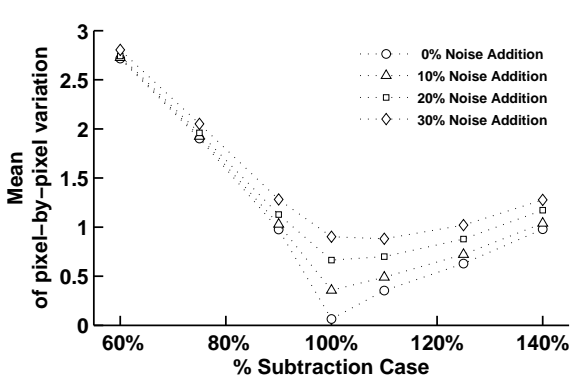

(b)

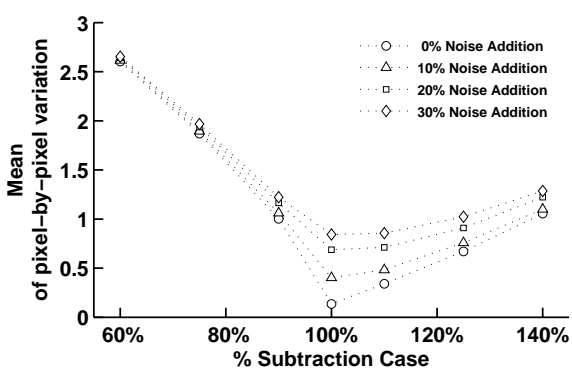

(d)

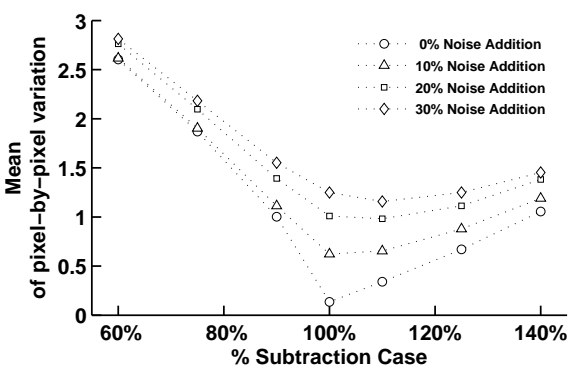

(f)

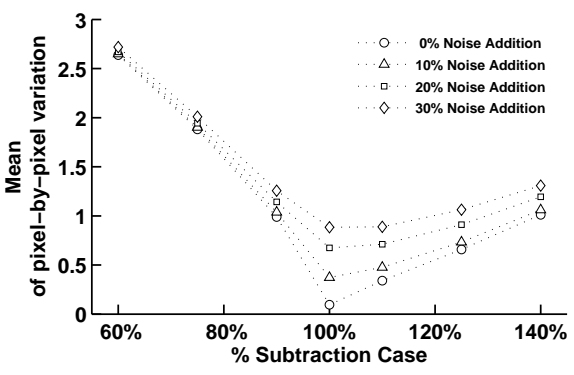

(h)

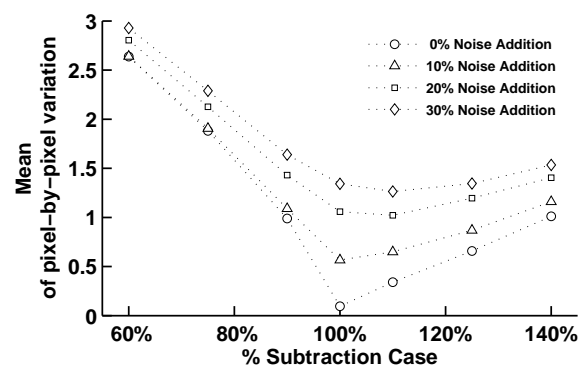

(i)

Figure 3.10: The mean of pixel-by-pixel variation versus subtraction case for all noise addition cases for distributions $1-9$. 
rates all four levels of noise addition, including the largest, 30\%, which would likely already result in a crude reconstruction. If only cases of $0 \%$ and $10 \%$ noise addition are considered, the minimum of the mean pixel-by-pixel variation plot obtains the correct subtraction value in every distribution.

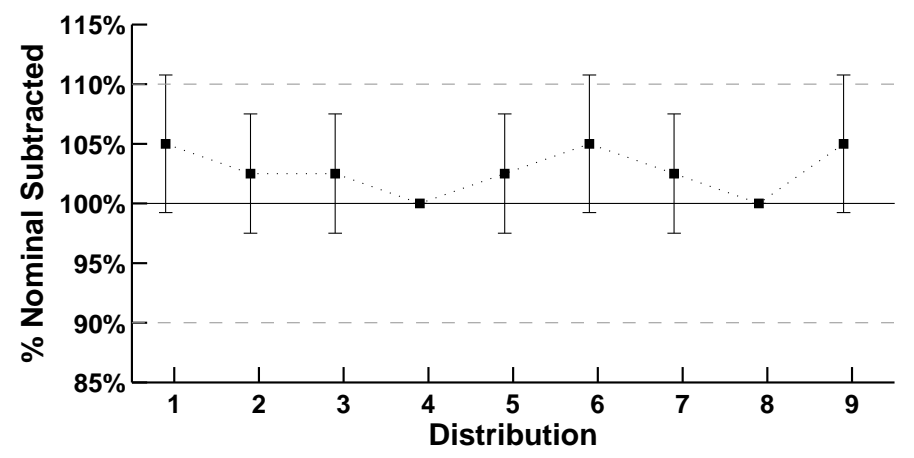

FIGURE 3.11: Summary of identification of correct background value for nine synthetic distributions.

\subsection{Impact of Background Subtraction}

The method of background subtraction described herein has shown that for nine simulated distributions with varying amounts of symmetry, dynamic range, and noise type and level, the actual value of the background can be determined to within $11 \%$ including all noise addition cases, up to 30\%. Given this method, it is desirable to assess the baseline total reconstruction error and compare this to the total reconstruction error resulting after background subtraction has been performed. Results will be presented for both $100 \%$ and $110 \%$ subtraction cases, as the higher noise addition cases may indicate an optimal subtraction value which varies slightly from the nominal value.

To assess the total reconstruction error, the 480 pixels within Region A are first smoothed using a Gaussian-derived weighted $5 \times 5$ smoothing function to reduce highfrequency non-physical noise, as would be applied to a reconstruction of experimental data. The relative error of these points in the region of interest is then calculated using their corresponding values in the actual distribution. For comparisons of distributions 
with noise addition, the actual distribution used for comparison is that which has the noise added. The absolute value of the relative errors for each pixel are then averaged. The calculation of relative error can occasionally be misleading if the "correct value" used in the calculation is very small. For distributions which have had noise addition, the "correct value" occasionally is very small. This generates a very large relative error, though the absolute error may be very small. Therefore any pixel value which has a relative error falling more than 1 standard deviations outside of the mean of relative error for that distribution are excluded. This procedure is first used to calculate the "baseline error", i.e. the average relative error of the original distribution, with $0 \%$ background subtraction, and a full reconstruction grid with an $84 \mathrm{~mm}$ radius and 5,544 grid cells.

Figure 3.11 provides the optimal value to subtract from the background, but does not immediately provide guidance on the optimal grid size to use. Figure 3.12 shows the mean relative error for all distributions at the baseline, $100 \%$, and $110 \%$ subtraction levels. For most distributions with $100 \%$ subtraction, there is a minimum value of mean relative error at the smallest grid radius. While this would be a tempting choice for a final grid reconstruction size, note that for distributions 4 and 7 , if the subtraction value is off by $10 \%$, the overall error is nearly doubled. If the targeted distribution for an experiment was expected to have very low noise (between 0-10\%) and the mean pixel-by-pixel plot had a very clear minimum, it would be ideal to use a small grid for reconstruction, to minimize mean relative error. However, this is not generally expected to be the case, and therefore a grid radius of $50 \mathrm{~mm}$ is selected for reconstruction, minimizing a majority of the relative error scenarios in Figure 3.12.

The mean relative error values of the various distributions at all four noise conditions at the $50 \mathrm{~mm}$ reconstruction grid radius are plotted in Figure 3.13a against the baseline case, and the overall \% improvement (as compared to the baseline case) is seen in Figure 3.13b. 


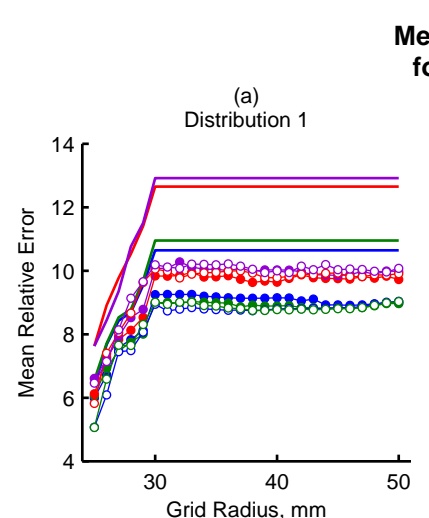

Mean Relative Error from Actual Distribution for baseline, $100 \%$, and $110 \%$ Subtraction
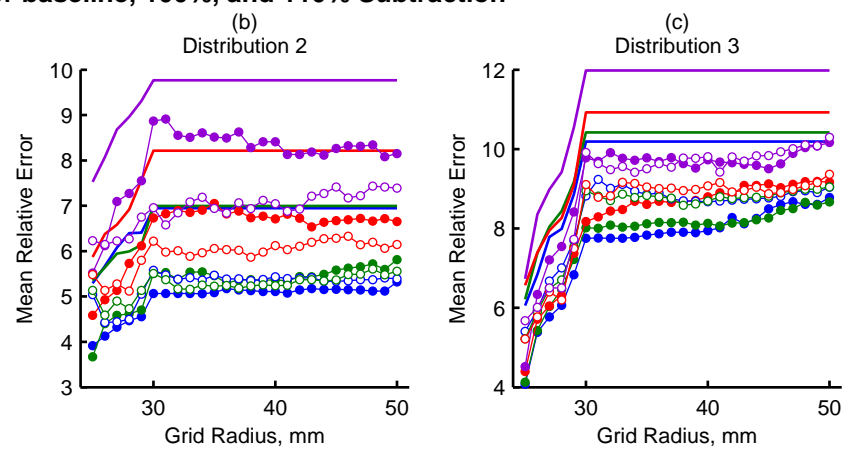

(d)

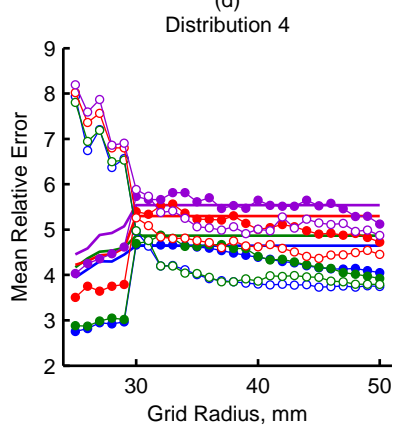

(e)
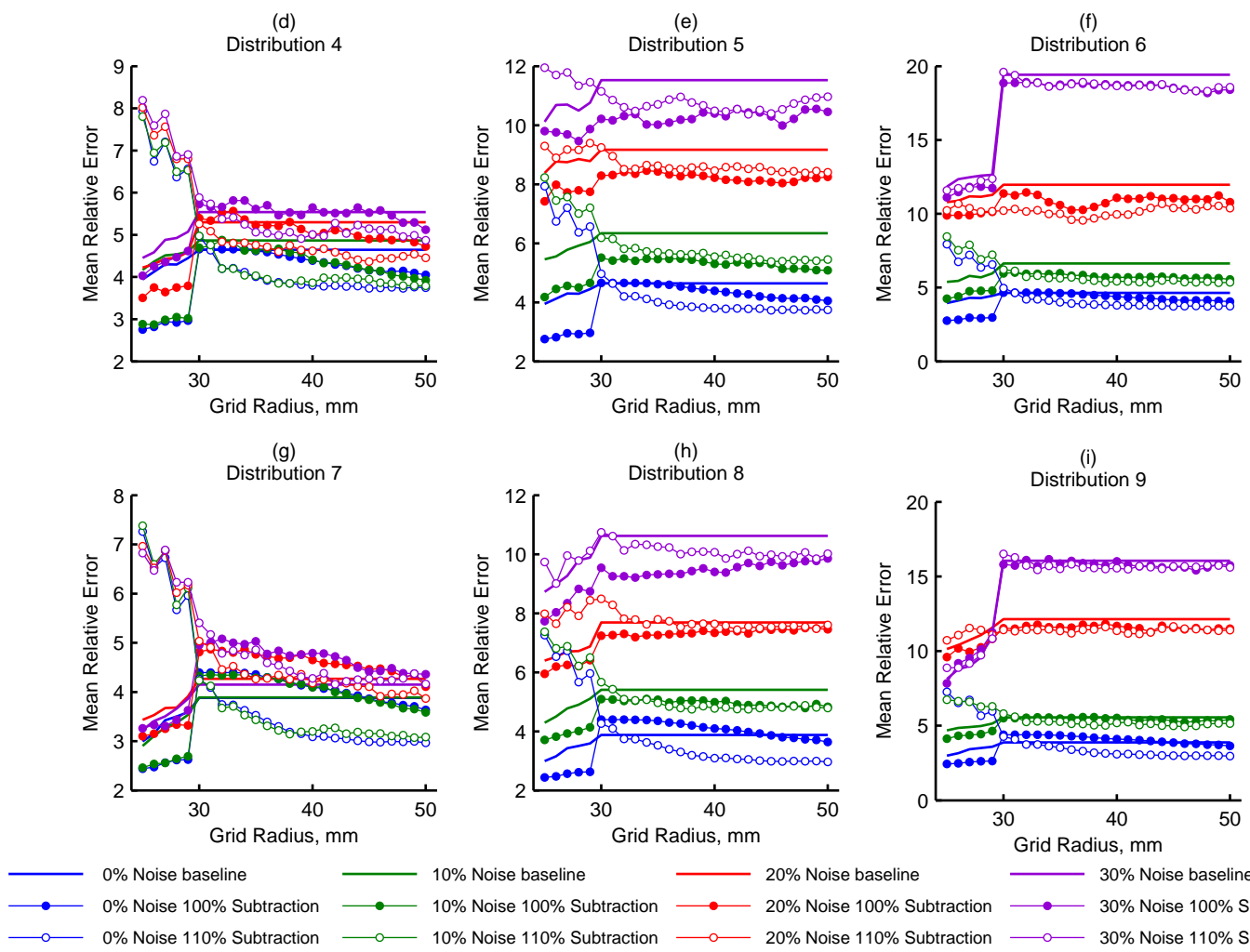

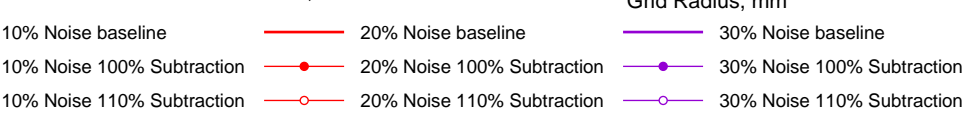

FiguRE 3.12: Mean relative error plots for the baseline, 100\% subtraction, and 110\% subtraction cases for all nine synthetic distributions as a function of reconstruction grid radius. 

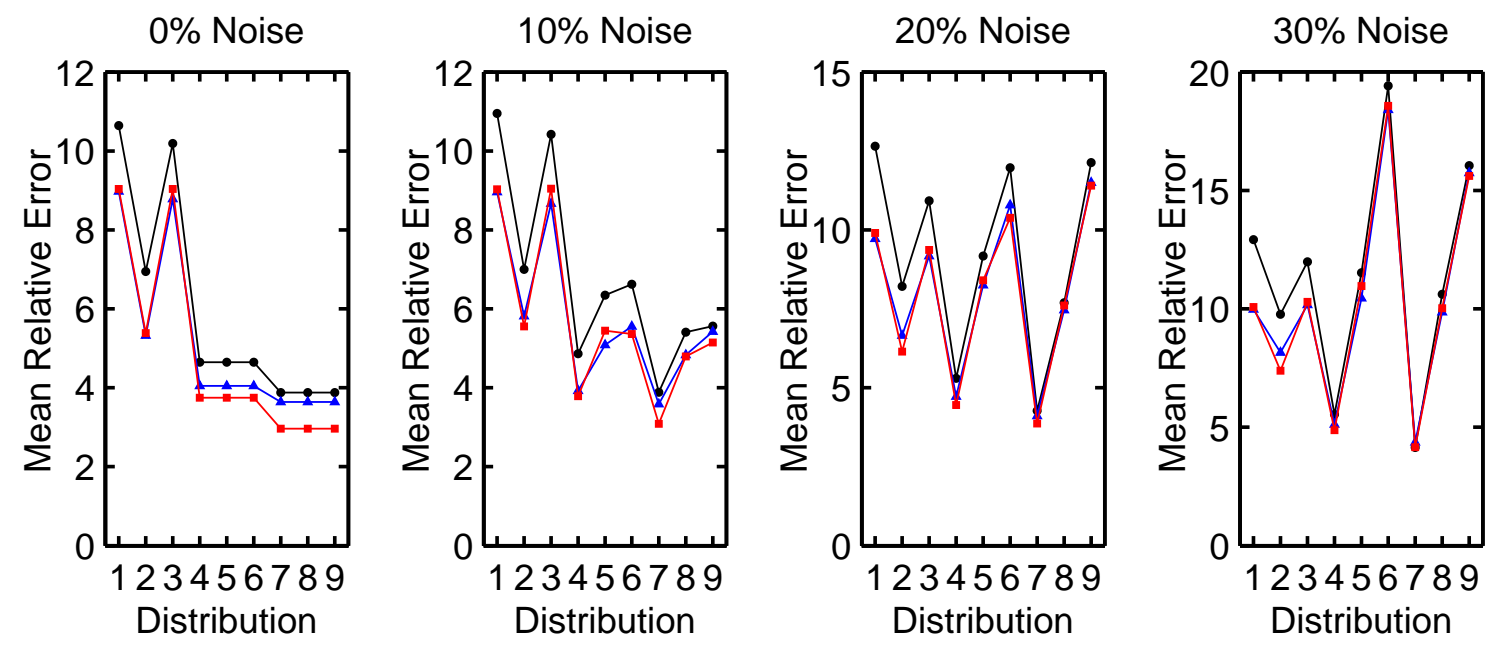

- No subtraction

$100 \%$ Subtraction

$\longrightarrow 110 \%$ Subtraction

(a)
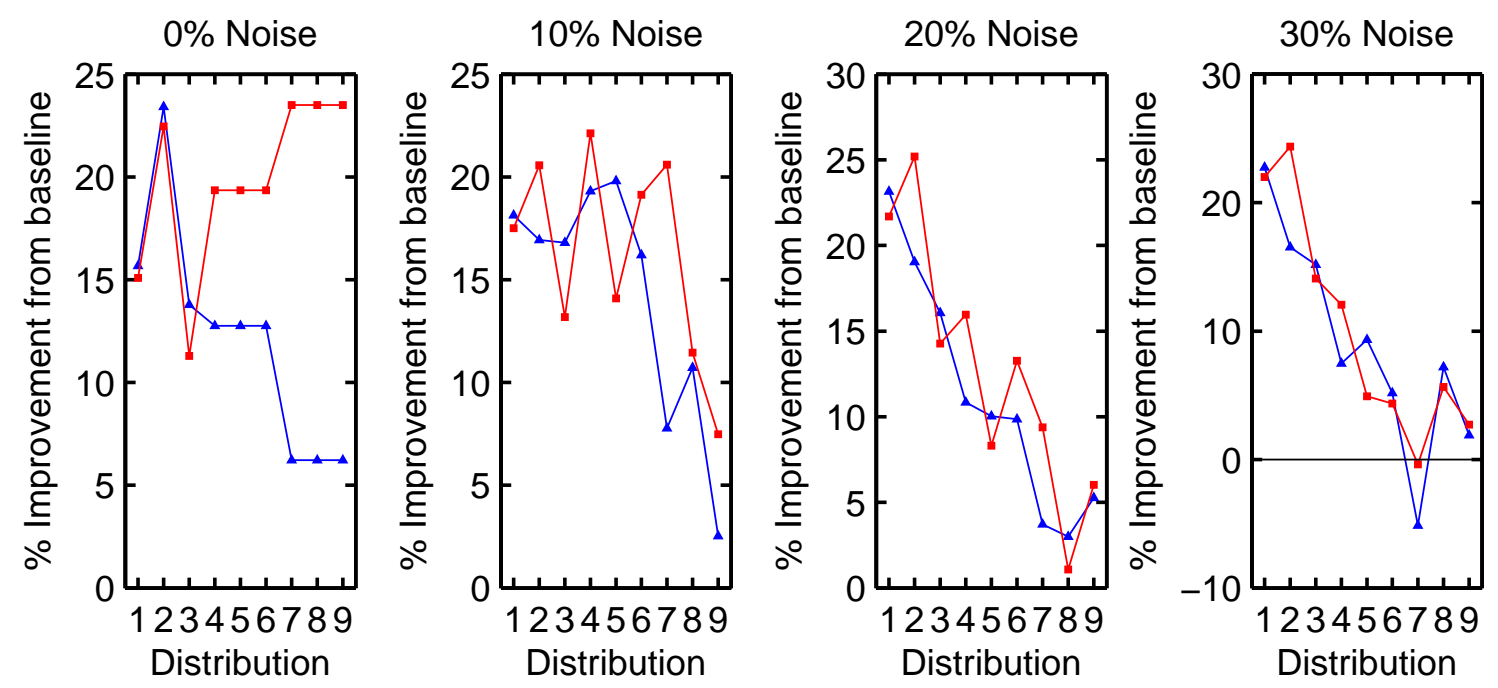

$\longrightarrow 100 \%$ Subtraction

$110 \%$ Subtraction

(b)

Figure 3.13: (a) Mean relative error at $50 \mathrm{~mm}$ reconstruction grid radius for baseline, $100 \%$ subtraction, and $110 \%$ subtraction cases, and (b) \% improvement over baseline case for $100 \%$ subtraction and $110 \%$ subtraction. 
It is observed that for all cases studied, the background subtraction method proposed improves the mean relative error of the reconstruction, with the exception of distribution 7 with $30 \%$ noise addition, which shows a slight detriment to the reconstruction. It is interesting that in several instances, the $110 \%$ subtraction actually improves the overall relative error of the reconstruction, though this is slight except in cases where there is $0 \%$ noise addition. Figure 3.13a allows for an estimation of overall reconstruction error depending on the estimated level of noise on the incoming sinograms. The mean of the relative error, calculated by averaging the error from each distribution, is summarized in Table 3.3.

TABLE 3.3: Summary of mean relative error for the baseline, 100\% subtraction, and $110 \%$ subtraction cases, at each level of noise addition.

\begin{tabular}{lllll}
\hline Noise addition level & $0 \%$ & $10 \%$ & $20 \%$ & $30 \%$ \\
\hline Baseline & 5.9 & 6.8 & 9.1 & 11.3 \\
$100 \%$ Subtraction & 5.1 & 5.8 & 8.0 & 10.2 \\
$110 \%$ Subtraction & 4.8 & 5.7 & 7.9 & 10.2 \\
\hline
\end{tabular}

When this method was applied to experimental data a global minimum was not readily obtained for each set of measurements. The resulting plots of mean pixelby-pixel variance for two sets of experimental data are shown in Figures 3.14a and 3.14b. As can be seen, in several instances, multiple minima were identified. In other cases, no minima were identified. The KB1 absorption showed minimums in each of the measurement sets this background subtraction method was applied to, but the resulting background absorption values were significantly different from each other, which was unexpected. Therefore, a simpler background subtraction method is utilized.

For synthetic data, a sinogram is simply the absorbance distribution multiplied by the area matrix, $A_{i j}$, and summed over each LOS. Therefore, it is observed that the edge-rays of a fan (the first few rows and last few rows of the sinogram) may have very little absorption originating from within Region A. These rays are identified in Figure 3.15 as black pixels, while rays which pass through Region A are shown as 
Case 1: 12\% Steam, Background Subtraction
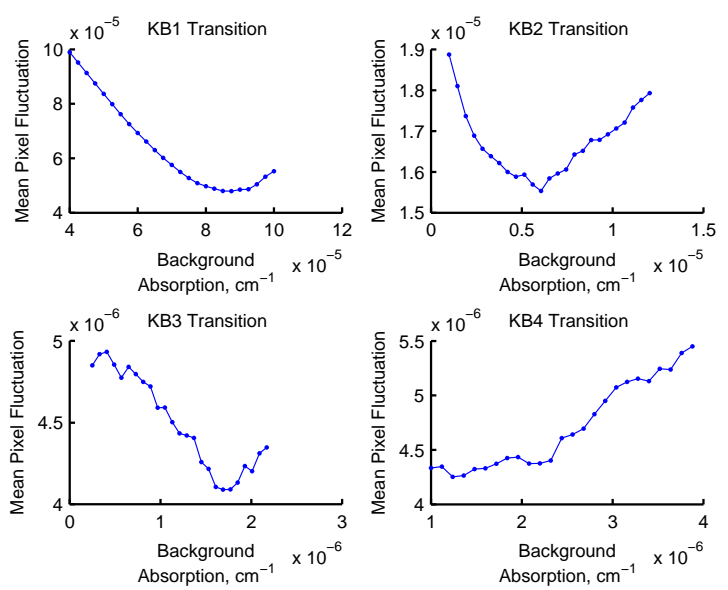

(a)

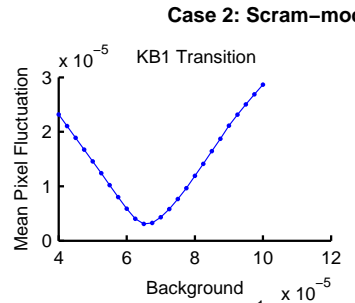

KB3 Transition
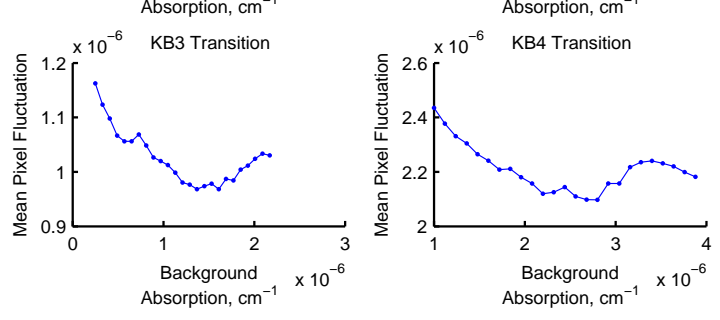

(b)

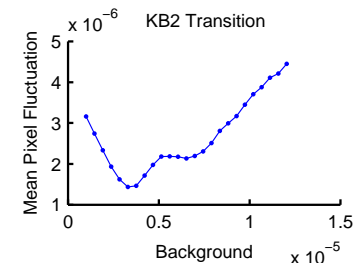

FiguRE 3.14: Mean pixel-by-pixel variance for background substitution attempt for (a) case 1 experimental data, and (b) case 2 experimental data.

white. The value of these edge rays, divided by the path length of the beam, equals far-field value, assuming it is constant.

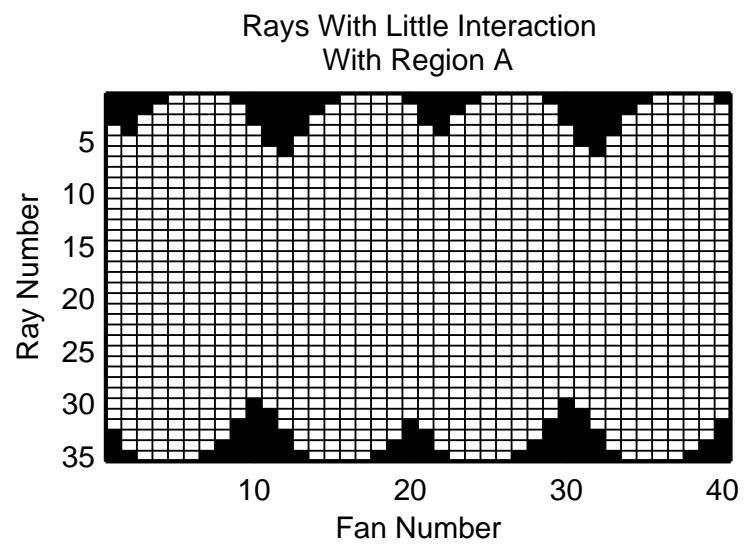

FiguRE 3.15: Black pixels represent LOS which have little interaction with Region A and white pixels represent LOS which intersect Region A.

The value of these edge rays, identified in Figure 3.15, and divided by the path length of the LOS are plotted in Figure 3.16 for distribution 2. The true value of this background is equal to three. Although cases which have high levels of noise addition show a bigger spread in values, it is seen that all cases are scattered with a mean about three.

The same analysis is performed for all nine synthetic distributions. The mean of 


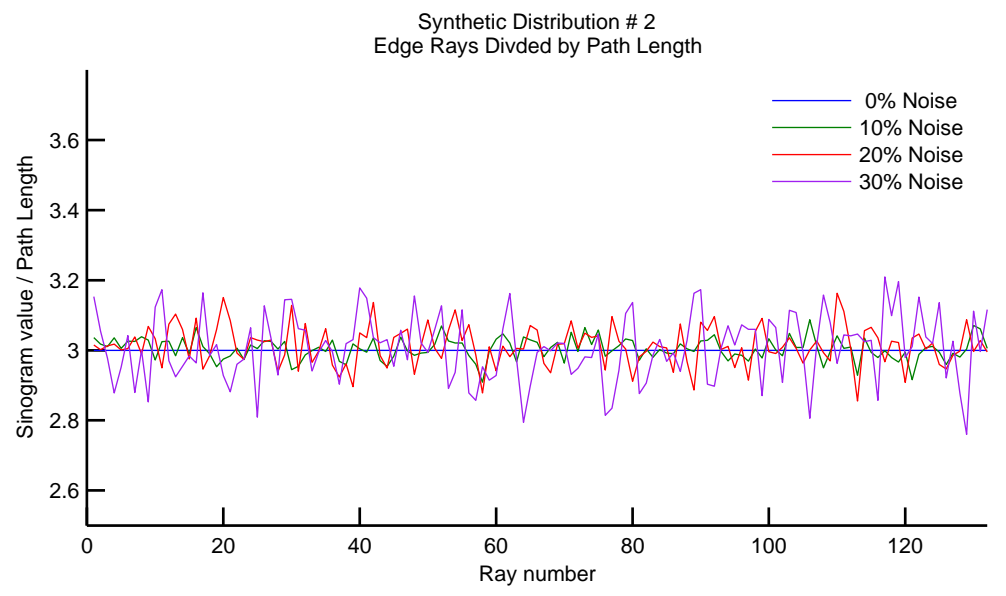

Figure 3.16: Edge rays divided by path length for synthetic distribution 2, for four cases of noise addition.

the identified background subtraction value and the standard deviation are shown in Figure 3.17. Distribution 1 does not appear in Figure 3.17a because its background value is of a different order of magnitude than the other distributions. For all distributions (including distribution 1), it can be seen that increasing the level of noise on the distribution increases the standard deviation of the identified background value, as to be expected. However, even if the worst case of noise is considered, $30 \%$ noise addition, the identified background value is still within $6 \%$ of the actual background value.

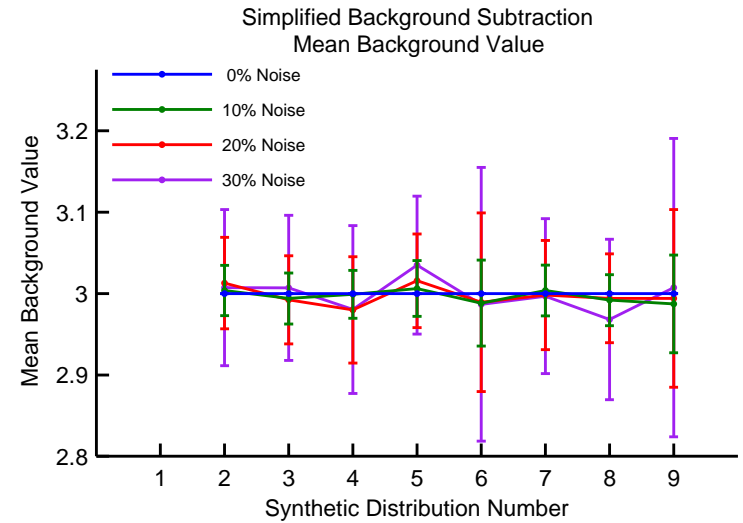

(a)

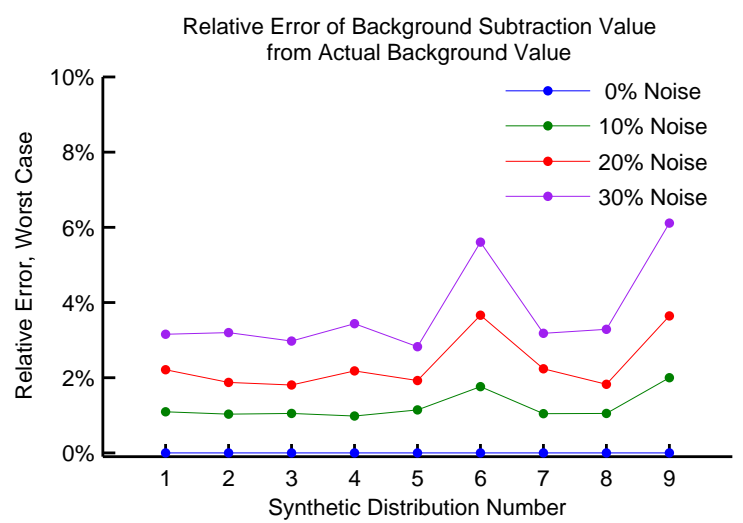

(b)

Figure 3.17: (a) Background subtraction values for the synthetic distributions identified via the simplified background subtraction method, and (b) relative error resulting from the worst-case guess of the background subtraction values. 
As the standard deviation for all of these simulations still falls within the $10 \%$ estimation of the "correct" background value subtraction, the final values of mean relative error of the resulting distributions (at baseline, 100\% of the "correct" value, and $110 \%$ of the "correct" value), shown in Table 3.3 are still valid.

This simplified method of background subtraction was used to identify the background absorbance values for each of the absorption features and for each of the experimental data sets. The resulting background absorbance values are shown in Figure 3.18 and tabulated in Table 3.4.

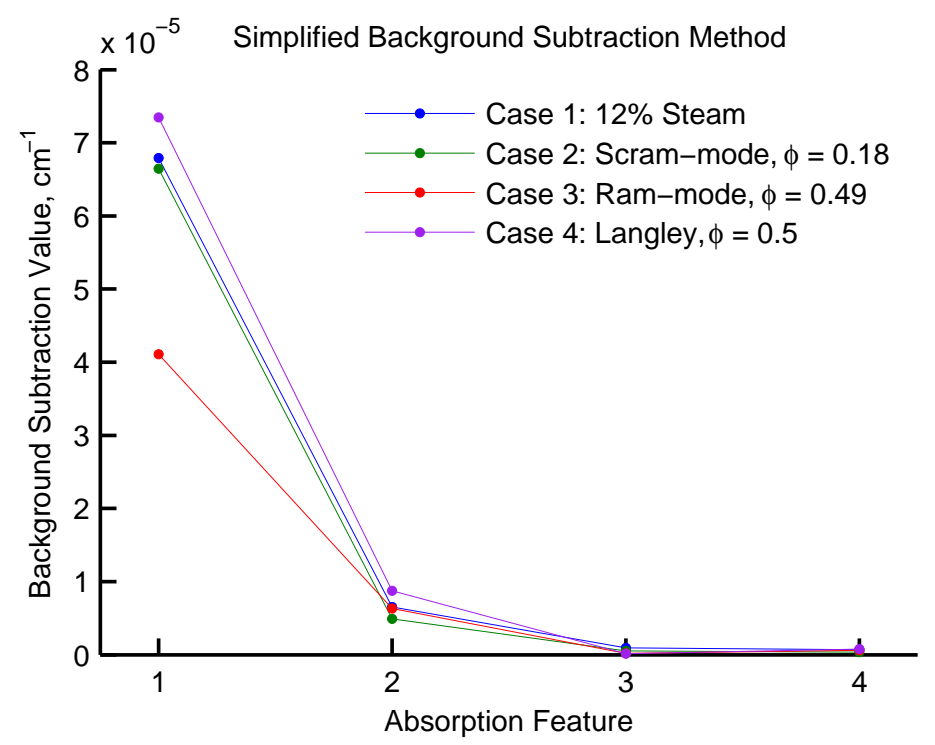

Figure 3.18: Resulting background absorbance values for each absorption feature and each experimental data set using simplified background subtraction method.

TABLE 3.4: Summary of background absorbance values for each absorption feature and each experimental data set.

\begin{tabular}{lllll}
\hline Experimental Data Set & \multicolumn{4}{c}{ Background absorbance value per pixel, $\mathrm{cm}^{-1}$} \\
& KB1 & KB2 & KB3 & KB4 \\
\hline Case 1: 12\% Steam & $6.7932 \times 10^{-5}$ & $6.5223 \times 10^{-6}$ & $9.2297 \times 10^{-7}$ & $6.7147 \times 10^{-7}$ \\
Case 2: Scram-mode, $\phi=0.18$ & $6.6441 \times 10^{-5}$ & $4.9191 \times 10^{-6}$ & $5.2395 \times 10^{-7}$ & $3.5318 \times 10^{-7}$ \\
Case 3: Ram-mode, $\phi=0.49$ & $4.1087 \times 10^{-5}$ & $6.3172 \times 10^{-6}$ & $2.0521 \times 10^{-7}$ & $6.0810 \times 10^{-7}$ \\
Case 4: NASA Langley, $\phi=0.5$ & $7.3456 \times 10^{-5}$ & $8.7373 \times 10^{-6}$ & $1.3503 \times 10^{-7}$ & $7.6685 \times 10^{-7}$ \\
\hline
\end{tabular}




\subsection{Uncertainty Analysis for TDLAT Measurements}

To assess the uncertainty in the TDLAT measurements, several hundred LOS measurements were collected at a single position. For a non-combusting case, 420 LOS measurements are collected at a single position. For a combusting case, 560 LOS measurements are collected at the same single position. This measurement allows for the calculation of the standard deviation of the integrated absorbance for each of the absorption features. The standard deviation divided by the average integrated absorbance gives the precision uncertainty for each signal. Assuming the errors are purely random and normally distributed, this quantification of the precision of the absorbance measurement can then be used with Table 3.3 to identify the mean relative error associated with that level of noise.

A spatial resolution of $2 \mathrm{~mm} \times 2 \mathrm{~mm}$ is utilized for cases 1,2 , and 3 , while a spatial resolution of $4 \mathrm{~mm} \times 4 \mathrm{~mm}$ is utilized for case 4 . These spatial resolutions are suitable for the TDLAT measurements on the assumption that the LOS measurements have a half-beam width less than $2 \mathrm{~mm}$ for cases 1,2 , and 3 and less than $4 \mathrm{~mm}$ for case 4 . A discussion of the reconstruction artifacts arising from this choice of spatial resolution was presented above and is incorporated into the tomographic reconstruction error, $\sigma_{\text {tomography }}$

A root-sum-square of the contributions of uncertainty is then calculated, with contributions from both the LOS uncertainty and the tomographic reconstruction error according to Equation 3.4.

$$
\sigma_{A}=\sqrt{\sigma_{L O S}^{2}+\sigma_{\text {tomography }}^{2}}
$$

The propagation of uncertainty from the reconstructed integrated absorbance to the temperature, number density, and mole fraction calculations via Boltzmann plot analysis are calculated according to Equations $3.5 \mathrm{a}-3.5 \mathrm{~m}$ as described by Brown et al. [37]: 


$$
\begin{aligned}
& \sigma T=\frac{\sigma b}{\frac{k_{B}}{h c} b^{2}}=\frac{1}{\frac{k_{B}}{h c}} \frac{\sqrt{S} \Delta^{1.5}}{(S S x y-S x S y)^{2}} \\
& \sigma N=\frac{Q(T)}{Q\left(T_{\text {ref }}\right)} e^{a} \sigma a \\
& \sigma X=\sqrt{\left(\frac{\partial X_{H 2 O}}{\partial T} \sigma T\right)^{2}+\left(\frac{\partial X_{H 2 O}}{\partial N_{H 2 O}} \sigma N\right)^{2}+\left(\frac{\partial X_{H 2 O}}{\partial P} \sigma P\right)^{2}} \\
& \sigma \text { Flux }=\sqrt{\left(\frac{\partial F l u x}{\partial N_{H_{2} O}} \sigma N\right)^{2}+\left(\frac{\partial F l u x}{\partial V_{x}} \sigma V_{x}\right)^{2}} \\
& \sigma \eta_{c}=\sqrt{\left(\frac{\partial \eta_{c}}{\partial \sum F l u x} \sigma \sum F l u x\right)^{2}+\left(\frac{\partial \eta_{c}}{\partial \dot{N}_{\text {Hydrogen,injected }}} \sigma \dot{N}_{\text {Hydrogen,injected }}\right)^{2}} \\
& S=\sum_{i=1}^{3} \frac{1}{\sigma_{i}^{2}} \\
& S x=\sum_{i=1}^{3} \frac{x_{i}}{\sigma_{i}^{2}} \\
& S y=\sum_{i=1}^{3} \frac{y_{i}}{\sigma_{i}^{2}} \\
& S x x=\sum_{i=1}^{3} \frac{x_{i}^{2}}{\sigma_{i}^{2}} \\
& S x y=\sum_{i=1}^{3} \frac{x_{i} y_{i}}{\sigma_{i}^{2}} \\
& \Delta=S S x x-(S x)^{2} \\
& a=\frac{S x x S y-S x S x y}{\Delta} \\
& \sigma a^{2}=\frac{S x x}{\Delta}
\end{aligned}
$$

where $\sigma T$ is the uncertainty in the temperature measurement, $\sigma N$ is the uncertainty in the number density measurement, $\sigma X$ is the uncertainty in the mole fraction calculation, $\sigma P$ is the uncertainty in the static pressure, $\sigma$ Flux is the uncertainty 
in the flux of water vapor, $\sigma \eta_{c}$ is the uncertainty in the combustion efficiency, and $S, S x, S y, S x x, S x y$, and $\Delta$ are various components of the uncertainty following the notation of Press et al. [56] $x$ and $y$ are the input parameters to the Boltzmann plot fit, defined earlier as $x_{\text {Boltzmann }}$ and $y_{\text {Boltzmann }}$, and $a$ and $\sigma a$ are the intercept (and uncertainty of the intercept) from the Boltzmann plot analysis, defined earlier as $c_{0} . \quad \sigma_{i}$ 's are the individual uncertainties of the integrated absorbance for each absorption feature, $\sigma_{A_{K B 1}}, \sigma_{A_{K B 2}}$, and $\sigma_{A_{K B 3}}$. Note that Equation 3.5b has omitted the $\frac{1}{L}$ factor, as once the absorbance fields have been reconstructed, the path length term has already been divided out. Actual error values will be presented in Chapter 4 alongside the corresponding results. 


\section{Chapter 4}

\section{UVa Results}

\subsection{Introduction}

The TDLAT technique has been under development at the University of Virginia for the purpose of applying the measurement technique at the exit plane of the University of Virginia Supersonic Combustion Facility (UVaSCF). Past work included preliminary design and proof of concept [31][49], with significant work focused on measurements in a flat flame Hencken burner. The promising results from the flat flame study led to initial measurements of a previous flowpath of the UVaSCF by Bryner in 2010. Measurements were collected both for a non-combusting steam injection condition, as well as clean-air combustion in only the scram-mode of operation, becoming the first highly-resolved tomographic absorption spectroscopy measurements of a scramjet flowfield. The results again showed significant promise, though the reconstructions suffered slightly from reconstruction imaging artifacts. This chapter details the most recent advances to the TDLAT system and its application to the UVaSCF. Results will be presented for three facility operation conditions, as will be discussed in the subsequent sections. 


\subsection{Experimental Overview}

\subsubsection{Facility}

The UVaSCF is a dual-mode combustion wind tunnel which has the unique ability to run continuously, making it ideal for flow field diagnostics. Also key is this wind tunnel's electrical air pre-heating system, which, unlike combustion heated tunnels, allows the flow to be free of combustion products, such as water vapor and carbon dioxide. These combustion products affect the scramjet performance and are referred to as vitiates. The Aerospace Research Laboratory's (ARL) facility has the ability to reproduce vitiation effects by introducing known amounts of steam and carbon dioxide into the clean air upstream of the test section. This allows for a comparison between actual flow conditions and experiments run with vitiated test gas. [57] A schematic of the dual-mode combustion tunnel is seen in Figure 4.1. Clean air is heated by a series of electrical resistance heaters, brought through a flow straightener into a Mach 2 nozzle, then finally through the test-section consisting of an isolator, combustor, constant-area section, and extender, where it is expanded to atmospheric pressure.

The UVaSCF was designed to be modular and therefore can be assembled in various configurations. Possible configurations are listed below. Configurations A, C, and E have been the focus of the NCHCCP and have been investigated extensively. The TDLAT results presented herein were collected on Configuration $\mathrm{C}$ with hydrogen fuel.

1. Configuration A: Mach 2.03 nozzle, combustor with un-swept ramp, extender

2. Configuration B: Mach 2.03 nozzle, constant-area section, extender

3. Configuration C: Mach 2.03 nozzle, isolator, combustor with un-swept ramp, constant-area section, extender

4. Configuration D: Mach 2.03 nozzle, isolator, combustor with un-swept ramp, extender 


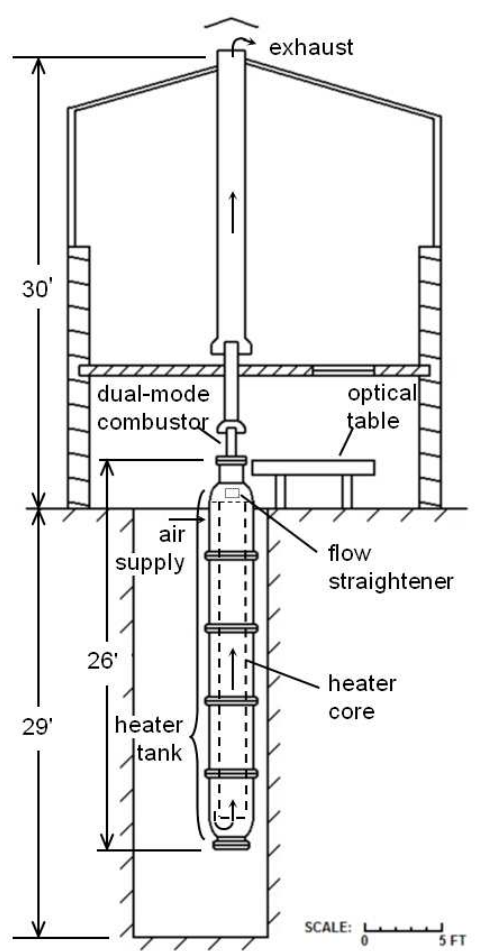

(a)

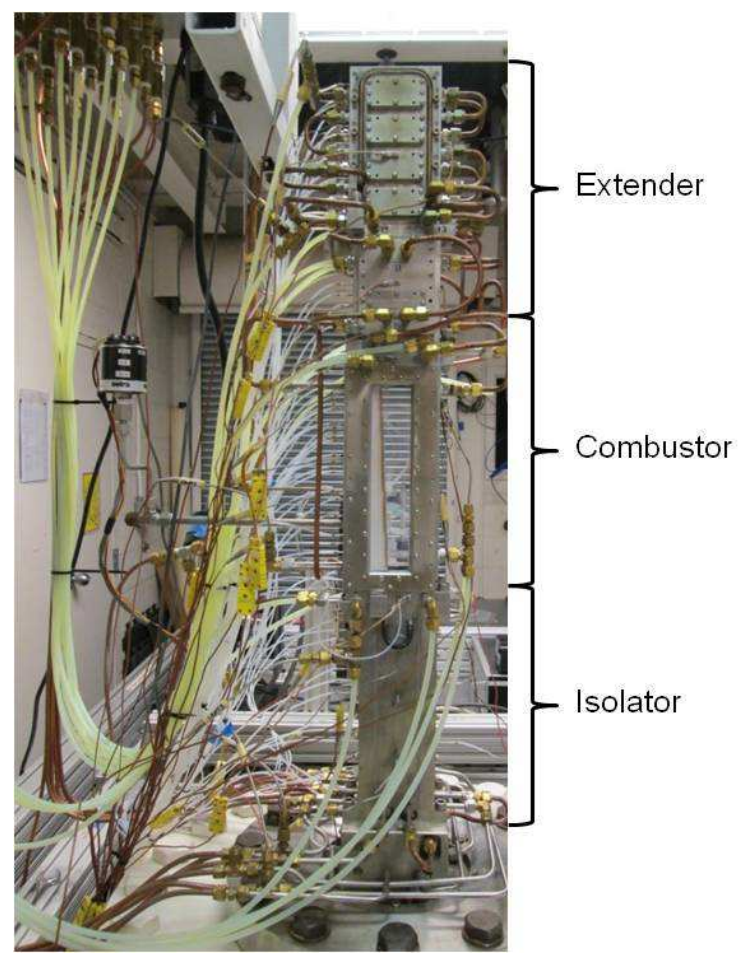

(b)

Figure 4.1: UVaSCF (a) schematic, and (b) test section hardware for Configuration C.

5. Configuration E: Mach 2.03 nozzle, isolator, combustor with cavity flame-holder, constantarea section, extender

TDLAT measurements were acquired for three facility operating conditions, as described in Table 4.1. These conditions are referred to as case 1, case 2, and case 3, operating at the conditions of "fuel-off", "scram-mode", and "ram-mode" respectively. The equivalence ratio, $\phi$, is defined as the ratio of the actual fuel-to-air ratio to the stoichiometric fuel-to-air ratio.

TABLE 4.1: Summary of UVaSCF operating conditions for TDLAT measurement collection.

\begin{tabular}{lllllll}
\hline $\begin{array}{l}\text { Case } \\
\text { Number }\end{array}$ & $\begin{array}{l}\text { Operation } \\
\text { Mode }\end{array}$ & $\begin{array}{l}\mathrm{H}_{2} \text { Equivalence } \\
\text { Ratio, } \phi\end{array}$ & $\begin{array}{l}\text { Injected } \mathrm{H}_{2} \mathrm{O} \\
\text { mole fraction, } \%\end{array}$ & $T_{0}, \mathrm{~K}$ & $p_{0}, \mathrm{kPa}$ & $\begin{array}{l}\text { Nozzle Mach } \\
\text { Number }\end{array}$ \\
\hline 1 & Scram-mode & - & $12.0 \pm 0.5$ & $1200 \pm 0.8 \%$ & $300 \pm 1 \%$ & $2.03 \pm 1 \%$ \\
2 & Scram-mode & $0.18 \pm 5 \%$ & - & $1200 \pm 0.8 \%$ & $300 \pm 1 \%$ & $2.03 \pm 1 \%$ \\
3 & Ram-mode & $0.49 \pm 5 \%$ & - & $1200 \pm 0.8 \%$ & $300 \pm 1 \%$ & $2.03 \pm 1 \%$ \\
\hline
\end{tabular}


The UVaSCF is instrumented with low-frequency static pressure taps along the centerline of the wall holding the fuel-injector. These pressure taps can be seen in Figure $4.1 \mathrm{~b}$ along the left-most wall, seen as thin clear plastic tubing. They measure the static pressure at the wall along the length of the flow path. Figure 4.2 shows representative pressure traces from the operational UVaSCF for the three facility conditions listed above in Table 4.1 .

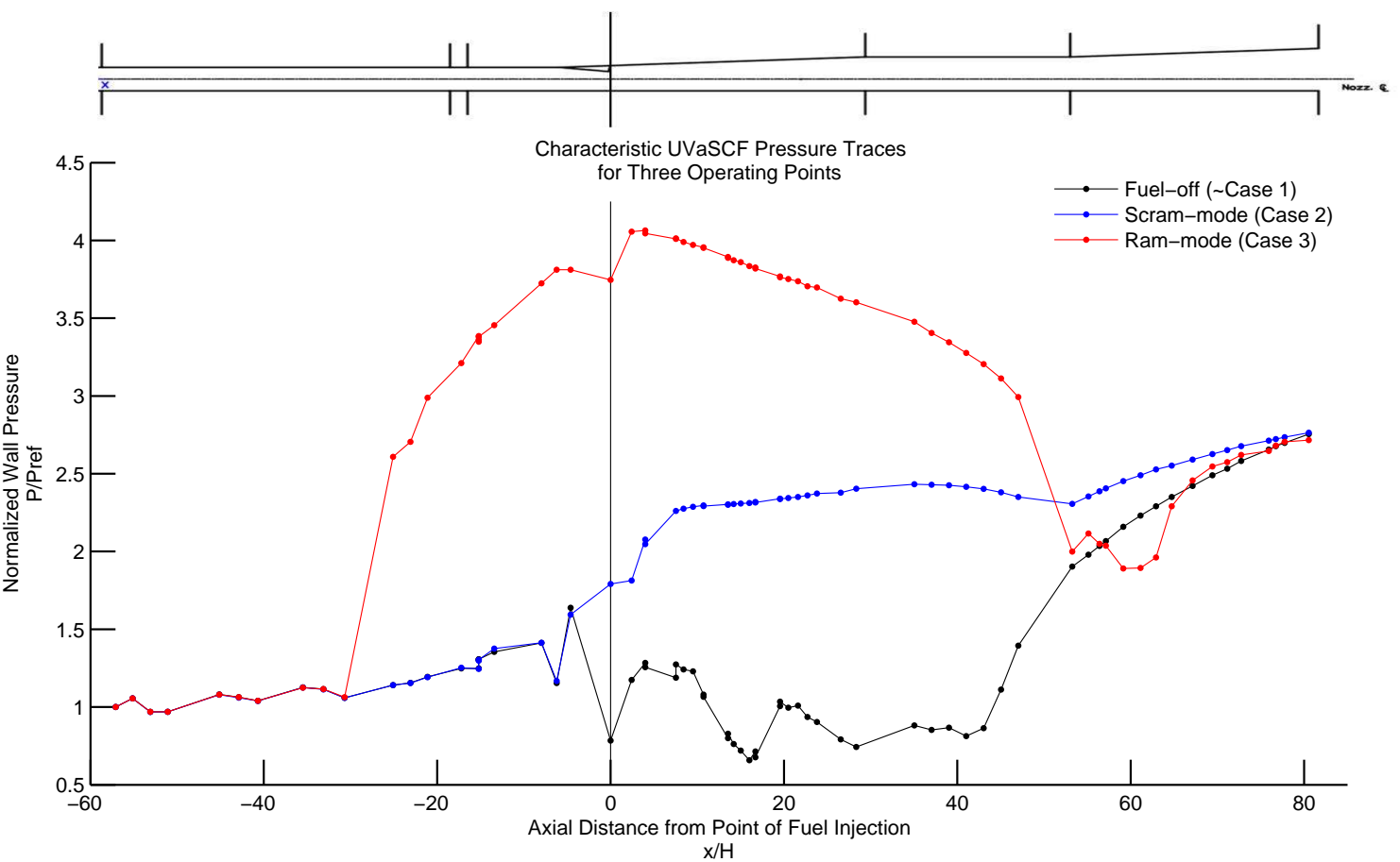

Figure 4.2: UVaSCF Configuration C flow path and characteristic pressure scans for three operating points.

The pressure trace for the fuel-off case, shown in black in Figure 4.2, shows the pressure variations when no fuel has been injected. The fuel-off condition shown here is very similar to the UVaSCF conditions for TDLAT measurements in case 1; however, this case has steam injected upstream of the flow path pictured. For the purpose of understanding the geometry of the UVaSCF flow path, the fuel-off pressure trace will suffice. The pressure is normalized by the pressure at the exit of the Mach 2.03 nozzle, typically $\sim 36 \mathrm{kPa}$. The $\mathrm{x}$-axis is the axial distance from the base of the ramp measured in $\mathrm{x} / \mathrm{H}$, or axial distance divided by the height of the ramp which 
equals 0.25 inches. $\mathrm{x} / \mathrm{H}$ of 0 represents the base of the ramp, where $H_{2}$ fuel is injected parallel to the face of the ramp.

For case 1, the pressure trace gradually increases from the exit of the nozzle to just upstream of the ramp. This gradual increase in pressure is indicative of a growing boundary layer, but signifies that the flow is attached and has not separated. The flow is supersonic throughout the isolator and combustor. After the ramp, because there is no fuel combusted in this case, the increasing area of the combustion section alleviates the pressure until $\mathrm{x} / \mathrm{H} \sim 40$. At this point, the pressure difference between the flowfield and the exit conditions ( 1 atmosphere) is nearly $3.5 \times$, forcing the flow to equilibrate through a series of shockwaves. This set of shockwaves decelerates the flow while increasing the static pressure and static temperature.

For case 2, the pressure trace is nearly identical to that of case 1 until right before the ramp. Again, the flow is supersonic through the isolator with a growing, but still attached, boundary layer. At the point of fuel injection, there is a rise in pressure due to the heat addition from the combusting $H_{2}$ fuel. Again, the pressure is below the atmospheric pressure at the exit, and thus the flow equilibrates through a series of shockwaves through the extender section. This mode of combustion is termed scram-mode, due to the supersonic speeds of the flow entering the combustor.

Case 3 shows the same pressure trace as the other cases up until $\mathrm{x} / \mathrm{H} \sim-30$. At this point, a large increase in pressure is observed upstream of the fuel injection due to pressure rise in the combustor. This is the leading edge of the pre-combustion oblique shock train which exists primarily in the isolator. This shock train decelerates a significant portion of the flowfield, but does not terminate in a normal shockwave, causing there to be mixed subsonic/supersonic flow in the combustor section. The pre-combustion shocktrain separates the boundary layer as is evident in the pressure trace. A thermal throat is observed at $\mathrm{x} / \mathrm{H} \sim 53$, where the flow has been choked due to the large amount of heat addition. The subsonic flowfield is then accelerated to sonic at this thermal throat. The thermal throat acts as a converging-diverging 
nozzle for a subsonic flow and would be expected to accelerate the flow to supersonic speeds downstream. However, again due to the adverse pressure gradient between the internal flowfield and the atmospheric pressure at the exit of the scramjet model, the flow is decelerated through a series of shockwaves until it reaches the exit plane and exhausts to the atmosphere. The operating point of case 3 is termed the ram-mode.

\subsubsection{Experimental Setup}

There are two different configurations in which the TDLAT experiment will be physically arranged: in the single-box configuration and multi-box configuration. The single-box configuration was initially developed by Bryner [31] and Snyder [49] for use on the UVaSCF. The upper image in Figure 4.3 shows the current single-box TDLAT configuration to be used with the UVaSCF. The improved system now allows for three lasers to be temporally multiplexed using a MEMS-type fiber optical switch system, where as Bryner's work allowed for only two lasers at a time. This reduces the length of the experiment significantly, from $2+$ hours to 40 minutes, while also preserving maximum power of the laser. After the three lasers are multiplexed, the beam is split with a $95 \% / 5 \%$ ratio to the TED 3.0 box and to a solid etalon, respectively. The solid etalon was pictured in Figure 2.15. The polarization maintaining (PM) optical fiber is connected to the single TED box as seen in Figure 2.9. The TED box is mounted on a Velmex B5990TS rotary table which allows it to rotate, creating a fanbeam. After a single fanbeam is collected, the RTM660 rotational ring allows the entire system to rotate to the next measurement position. In the past, measurements have been collected using 72 fans (spaced $5^{\circ}$ apart), each consisting of 25 rays (spaced $1^{\circ}$ apart), and took on the order of 1 hour to collect a data set. [31] As mentioned previously, the TDLAT system used by Bryner could only employ two lasers at a time, and thus if three lasers were desired, the experiment would be conducted twice resulting in test times of approximately two hours.

This experiment utilized the third generation TED box, with improved alignment 


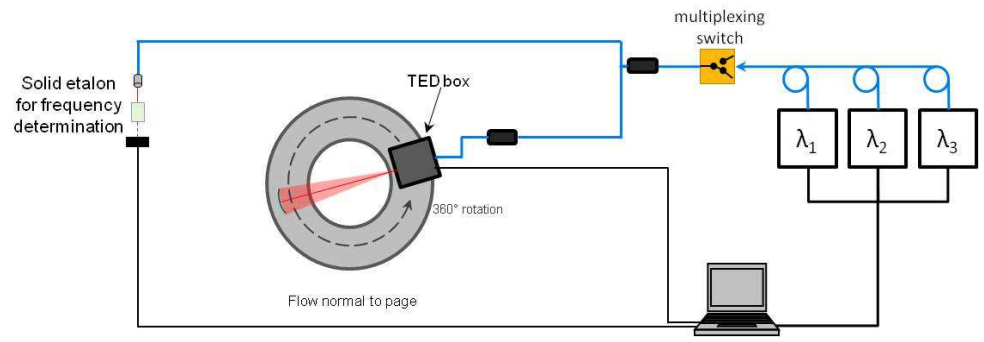

(a)

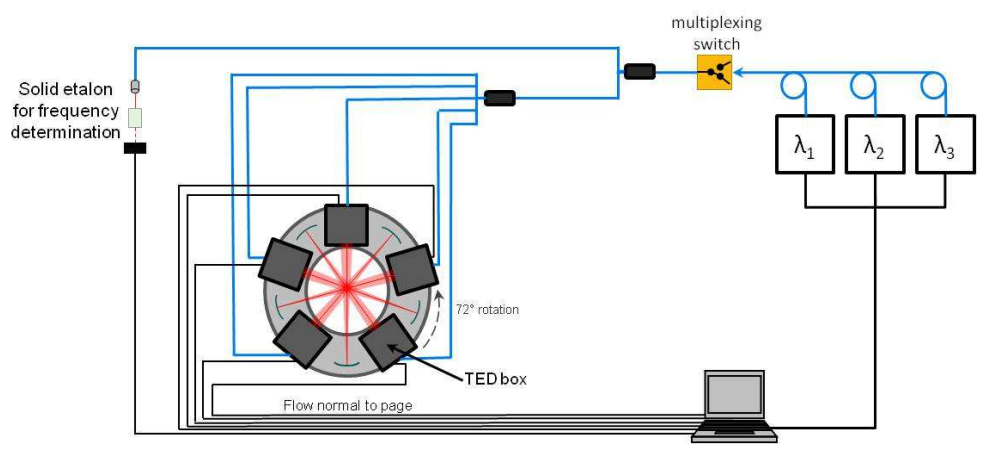

(b)

FIGURE 4.3: Schematic of (a) single-box configuration for TDLAT measurements on UVaSCF facility, and (b) multi-box configuration for TDLAT measurements on NASA Langley DCSCTF facility.

access and adjustability. Due to geometric constraints of the UVaSCF hardware, the TED box was mounted on a Velmex rotational stage upside down, while rigidly connected to the Newport rotational stage below, seen in Figure 4.4. This allowed for TDLAT measurements to be obtained at a measurement plane $6 \mathrm{~mm}$ downstream of the exit of the extender. This is the same plane at which SPIV measurements have been collected. The measurement plane was selected to be slightly downstream of the UVaSCF exit because the facility's test section experiences marginal thermal growth during operation. The TDLAT system was mounted to the facility's heater tanks, but not to the test section itself, and therefore did not translate with the test section during thermal growth. Therefore, a plane $6 \mathrm{~mm}$ downstream of the actual exit (before tunnel operation when the test section is cold) was selected to avoid the loss of signal due to facility hardware in the laser's line-of-sight.

The TDLAT experiments performed at the exit plane of the UVaSCF fulfill Research Objectives I, II, and III, as defined previously in Chapter 1. The TDLAT mea- 


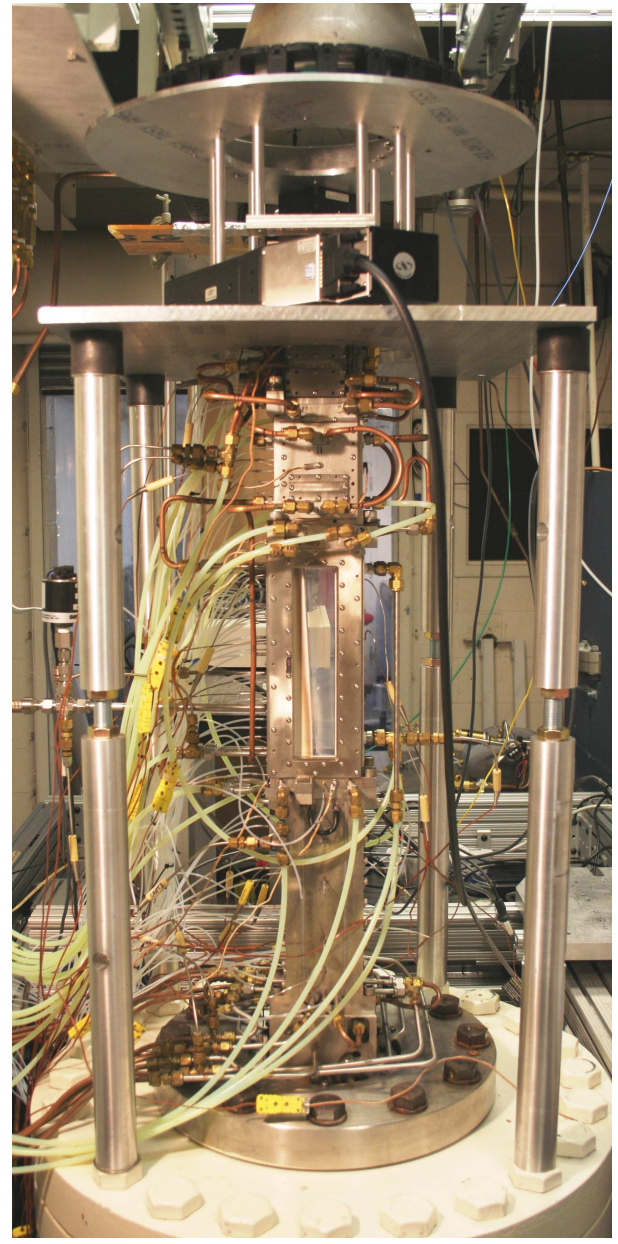

(a)

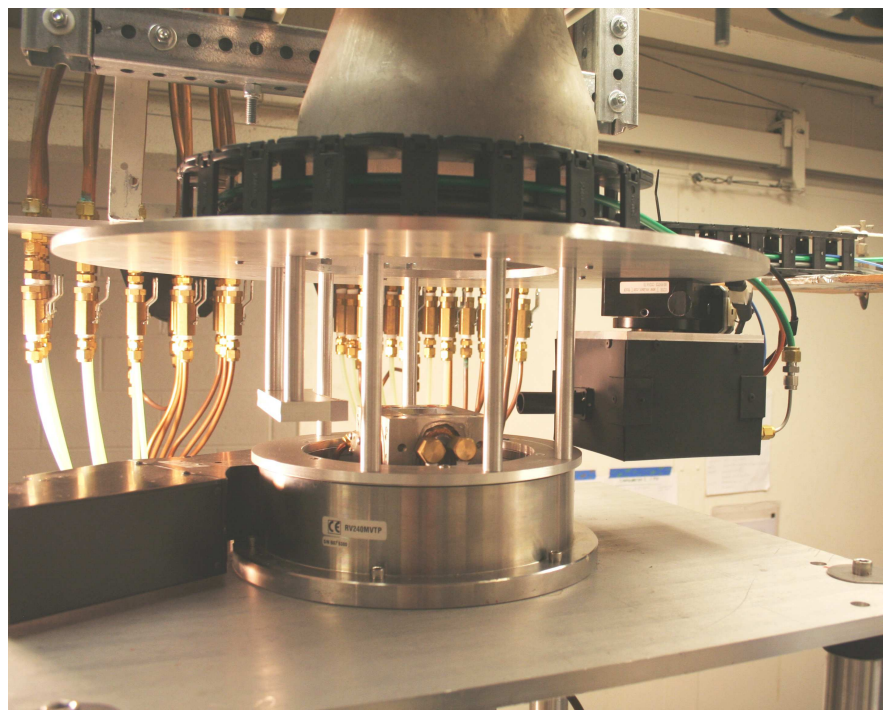

(b)

Figure 4.4: TDLAT hardware mounted on UVaSCF (a) full view, and (b) close up view at measurement plane. 
surements of the UVaSCF enrich one of the most complete experimental databases of a dual-mode scramjet facility under the NCHCCP to be used for the development and validation of CFD models, both near and long term.

\subsubsection{Calibration of Physical Orientation}

In order to map the reconstructed distributions into physical space, the TDLAT system recorded measurements with various solid objects placed at known locations within the field of view of the TDLAT experiment. As seen in Figure 4.5, two different distributions were considered. First, seen in Figures 4.5a, 4.5c, and 4.5e, two cylindrical posts were attached to two of the inner corners of the UVaSCF exit hardware. These posts were long enough such that they completely spanned the height of beam. A full set of tomographic measurements were collected and are seen in Figure 4.5e. This implementation can be considered a "binary" version of TDLAT, because either the beam's path is blocked by the solid object or it is not, allowing light to reach the photo detector.

The black pixels seen in the sinogram in Figure 4.5e correspond to the beam being completely obstructed (a solid object lies within that LOS), and the white pixels corresponding to the beam being completely unobstructed. The reconstruction of this measured binary sinogram, seen in Figure 4.5c, shows the white field (open space) with two black cylindrical features. The color range of this reconstruction has been windowed to enhance the clarity of the solid objects. The blue axes in the reconstruction represent the coordinate system which is centered in the reconstruction grid, with the corresponding size and shape of the UVaSCF exit plane hardware shown as a blue rectangle. Due to imperfect installation of the TDLAT measurement hardware, the origin of the TDLAT hardware (and the reconstruction grid) was not necessarily centered with reference to the actual UVaSCF exit plane flowpath. By shifting the axes (green) until the reconstructed cylinders were located within the tunnel hardware boundaries, it can be seen that the reconstruction grid needed to be 
shifted left by $4 \mathrm{~mm}$ along the y-axis. No shift in the z-direction was required.

This mapping is repeated with a different distribution of objects, seen in Figure 4.5b. Two bolts were threaded into existing holes in the UVaSCF exit hardware, which have well-known locations. A third object, a cylinder, was placed in the leftnear corner, tangent to the inner corner of the flow path boundary. The measured binary sinogram and reconstruction of this asymmetric distribution are seen in Figures 4.5f and $4.5 \mathrm{~d}$ respectively. Note that these objects have been placed outside of the primary measurement area of the UVaSCF (Region A), which is the circle inscribed by the UVaSCF flow path boundary at the exit. The sinogram confirms this, as it can be seen that the black "waves" in the sinogram (each representing one of the objects) extends past the limits of the sinogram. The resulting distribution is slightly suboptimal, and as such the reconstruction suffers. Though the reconstruction does not capture the shape of the solid objects perfectly, the same lateral shift of $4 \mathrm{~mm}$ to the left is found to align both the bolts to their known thread locations (red circles) and the cylinder to contact the lower-left corner of the flow path boundary. This physical mapping is an original contribution of the author and has never previously been employed in the TDLAT technique. This mapping allows for accurate comparisons of TDLAT results to CFD calculations, with objective alignment of the two fields.

Figure 4.6 depicts the orientation in which all subsequent results will be presented. Note that the orientation has been rotated $90^{\circ}$ clockwise compared to all earlier depictions of the UVaSCF exit plane. This is for consistency with the orientation of other presentations of measurements and CFD on the UVaSCF. It can be seen that the wall which contains the ramp fuel-injector (the lower wall in Figure 4.5) diverges at a $2.9^{\circ}$ angle. Therefore, compared to its position at the exit, the fuel injector is seen to be considerably further in-stream in the y-direction. The origin of the axes have been selected such that the center of the UVaSCF flowpath at the exit plane is $(0,0) \mathrm{mm}$. 


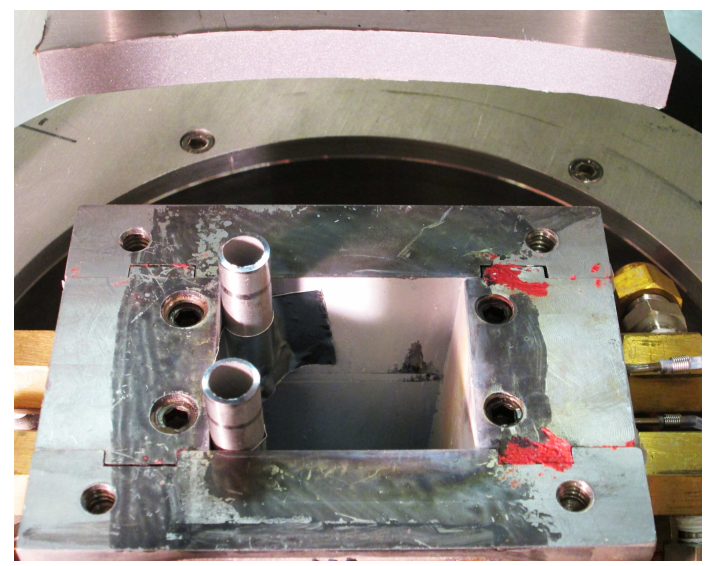

(a)

UVaSCF Mapping to Physical Space: Internal Dimensions

Reconstruction

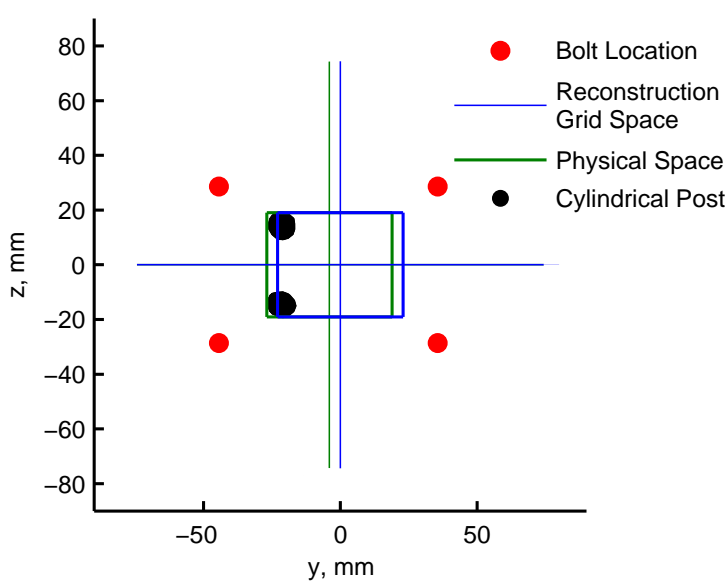

(c)

UVaSCF Mapping to Physical Space: Internal Dimensions

Sinogram

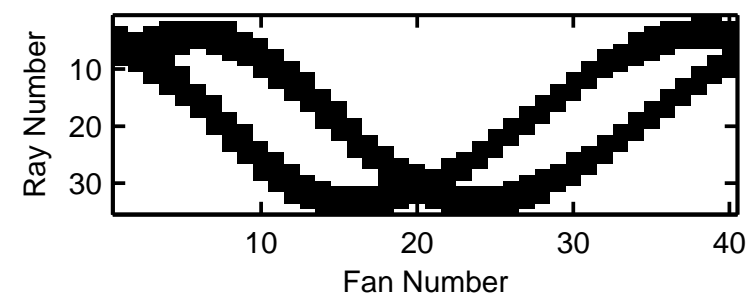

(e)

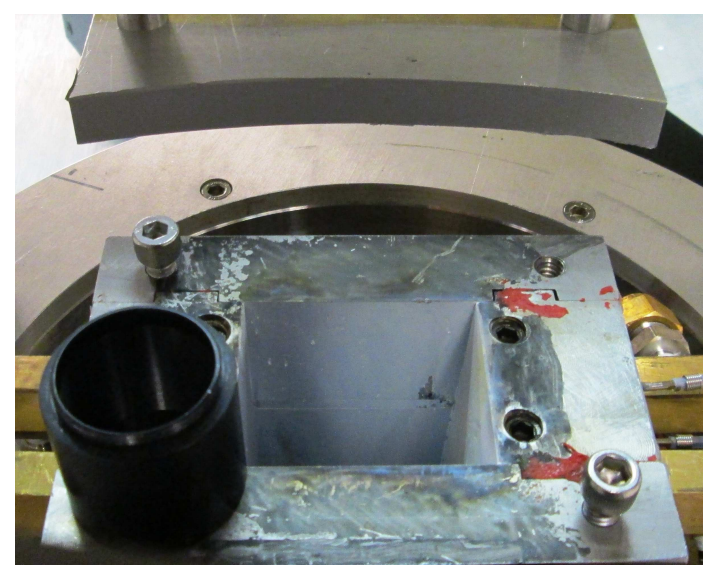

(b)

UVaSCF Mapping to Physical Space: External Dimensions

Reconstruction

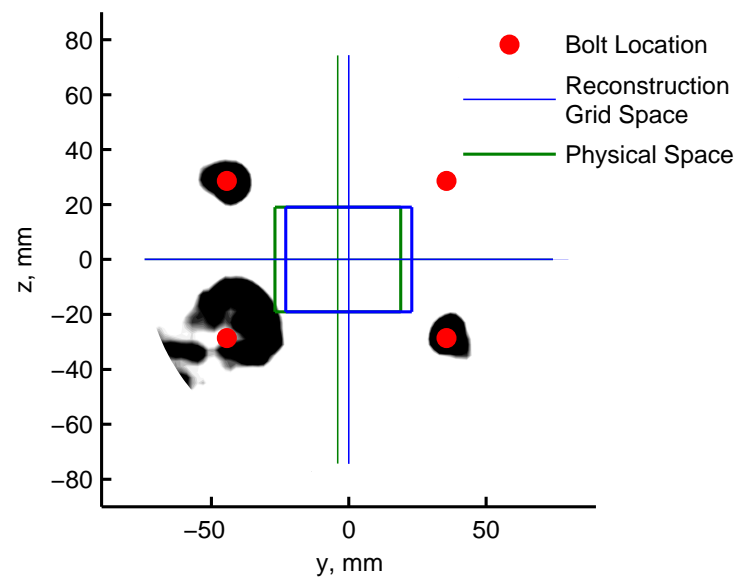

(d)

UVaSCF Mapping to Physical Space: External Dimensions Sinogram

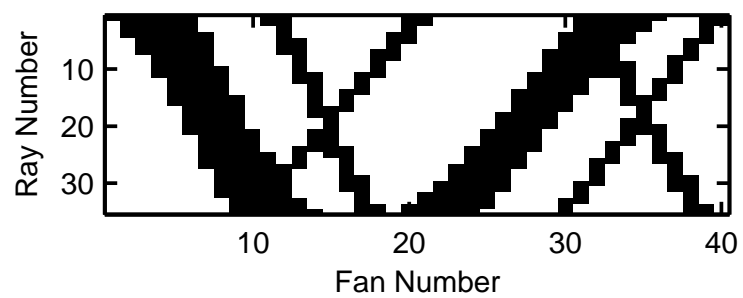

(f)

FiguRE 4.5: Mapping from reconstruction grid space to physical space with solid objects both inside and outside the tunnel flow path hardware. The first column shows (a) a photograph, (c) reconstruction, and (e) sinogram of the mapping of objects placed within the flow path. The second column shows (b) a photograph, (d) reconstruction, and (f) sinogram of the mapping of objects placed outside of the flow path. 


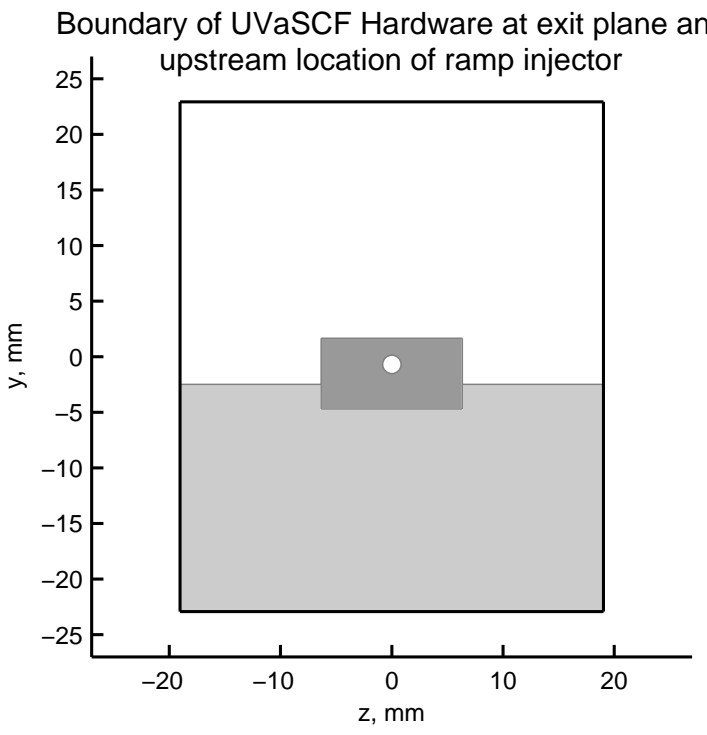

(a)

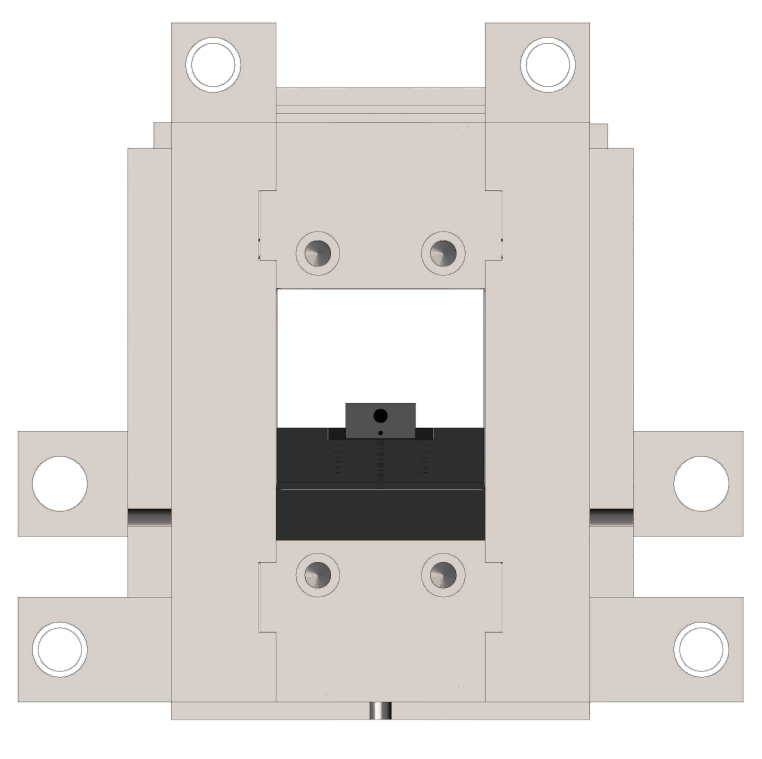

(b)

Figure 4.6: Orientation of the presented data to the actual UVaSCF. View is looking from above, with flow coming out of the page. The fuel injector is on the lower horizontal wall. (a) The view in which data will be presented, and (b) SolidWorks model of UVaSCF containing entire flow path.

Measurements were collected utilizing the TED 3.0 design shown in Figure 2.9. All four absorption features were collected in a temporally-multiplexed mode. Due to limitations in on-board memory of the data acquisition computer, measurements were collected in 20 sets, each collecting two fans. For example, the first fan of data was collected at $0^{\circ}$ (parallel to the fuel-injection wall, beginning from the left side of Figure 4.6 and traversing left-to-right. In the first fanbeam of this two-fan set, the rays pan from left-to-right, which is represented in the sinogram as rays $1 \rightarrow 35$. The Newport rotational ring then rotates clockwise (when viewing the UVaSCF from above as in Figure 4.6) $\Delta \theta$, which is equal to $9.0^{\circ}$ for data collected on the UVaSCF, seen in Table 2.1. To minimize the time of data collection, the rays collected in the second fanbeam of this two-fan set pan right-to-left, starting in its right-most position. The reversal of the order of these rays is accounted for, and they are organized into the sinogram as rays $35 \rightarrow 1$. 


\subsection{Case 1: 12\% Steam Results}

The first data case presented is one in which no fuel was injected, but instead steam was injected into the plenum far upstream of the UVaSCF test section. Due to the TDLAT technique's reliance on detectable water vapor, the UVaSCF's steaminjection capability is advantageous. Steam is injected via a sonic orifice near the bottom of the heater tank, seen in Figure 4.1a. The mass flow rate of the injected steam into the plenum can be calculated with the diameter of this sonic orifice, the discharge coefficient of the choked nozzle, the measured pressure and measured temperature upstream of the orifice. Though the UVaSCF does have the capability to also inject "make-up" oxygen (i.e. additional oxygen to correct the mole-fraction of the incoming air to be approximately $78 \% \mathrm{~N}_{2}$ and $21 \% \mathrm{O}_{2}$ ), this option was not used for this test case, as generally make-up oxygen is utilized for combustion applications when the amount of oxidizer is critical. Table 4.1 shows the test conditions for data collected in case 1 on August 23, 2013.

Figure 4.7 shows the measured sinograms for all four absorption features. These sinograms, along with all subsequently presented sinograms, are displayed prior to the background subtraction procedure. The figures in the left column show the measured data, with no adjustments. The figures in the right column show the corresponding sinograms, with the outliers filtered. The outliers of each sinogram have been addressed using a median filter with a $3 \times 3$ window. To accurately account for the first and last columns of the sinogram, each sinogram was first tiled horizontally, filtered, and then the central sinogram is utilized for reconstruction. The filtering of the sinograms can be performed on the tiled sinograms because of the periodic boundary condition, i.e. the $40^{\text {th }}$ fan is simply a rotation of $\Delta \theta$ away from the $1^{\text {st }}$ fan.

The quality of the KB1 and KB2 sinograms are seen to be excellent, even before filtering, as seen in Figures 4.7a and 4.7c. For consistency, they are filtered in the same manner that the KB3 and KB4 transitions' sinograms are filtered. It is notable 


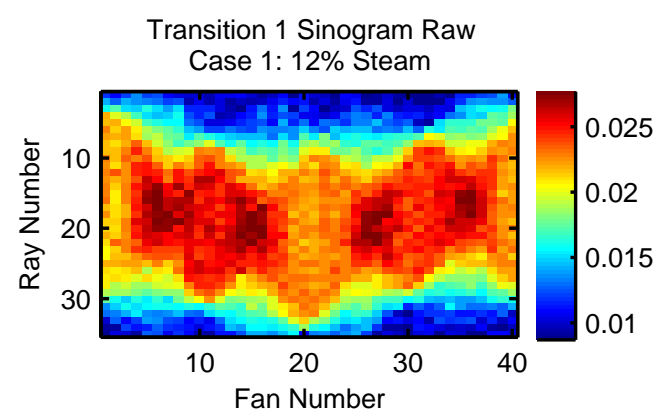

(a)

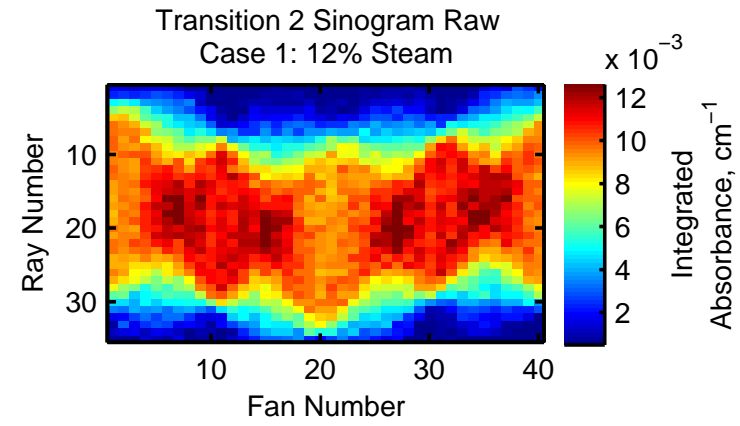

(c)

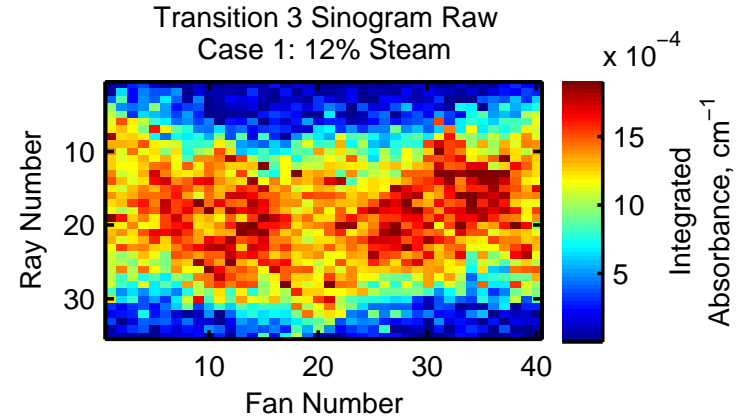

(e)

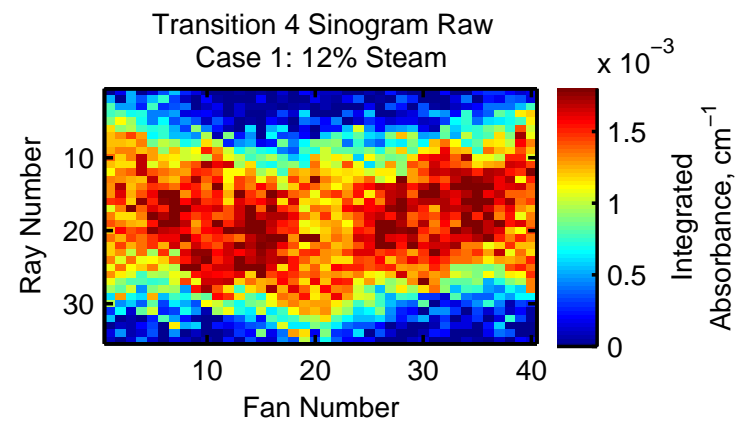

(g)

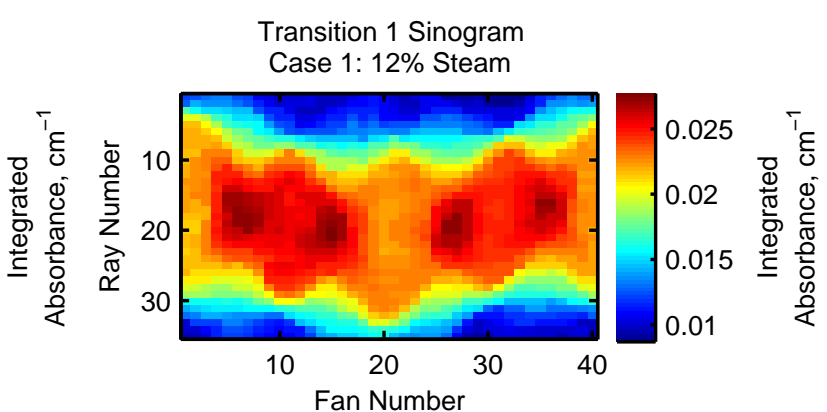

(b)

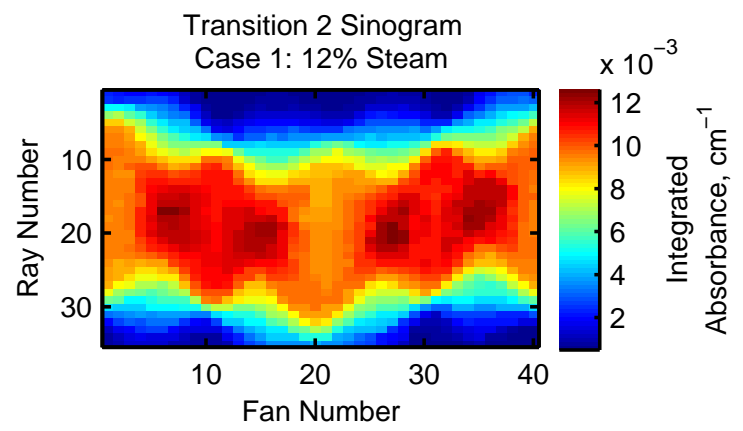

(d)

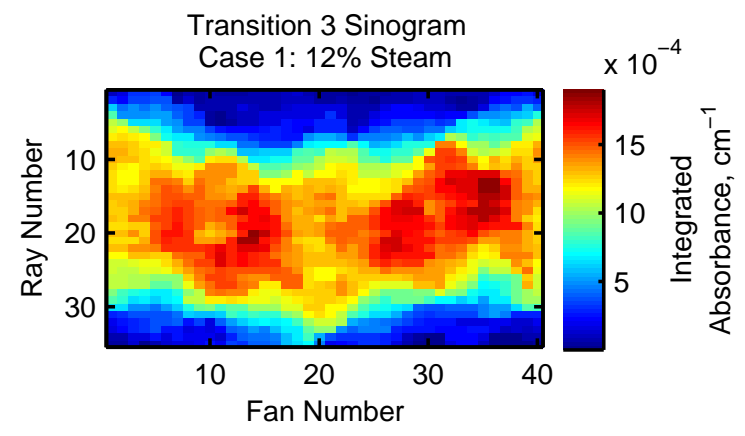

(f)

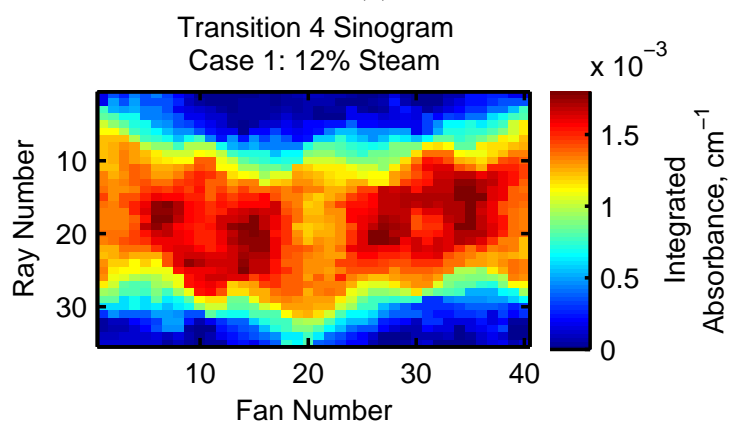

(h)

FIGURE 4.7: Integrated absorbance sinograms at the exit plane of the UVaSCF for case 1. The first column contains the raw sinograms of TDLAT measured integrated absorbance for each of the four absorption features (KB1-KB4) and the second column contains the corresponding filtered sinograms. 
that the shapes of Figures $4.7 \mathrm{~b}$ and $4.7 \mathrm{~d}$ are incredibly similar to each other, which is not apparent in the other data cases studied (as will be shown in subsequent sections). This suggests that the KB1 and KB2 reconstructions will also be very similar to each other. It should be observed, however, that the dynamic range (DR) of Figures 4.7b and $4.7 \mathrm{~d}$ differ significantly, with the DR of KB1's sinogram equal to 3, while the DR of KB2's sinogram much larger, at 12. These DR are still smaller than those of the KB3 and KB4 transitions, both $~ 18$, agreeing with the findings of Chapter 2 .

When Phase I analysis is completed for each of the absorption features, the 1-D path-integrated LOS temperature can be calculated for each ray in the sinogram via the Boltzmann plot method, creating a sinogram of LOS temperatures. While this LOS temperature sinogram is not to be reconstructed, it can give insight into the quality of data as well as demonstrate the large quantity of TDLAS data that has been collected. The actual quantity that is summed along a LOS is the absorbance of light due to the presence of water molecules. While this absorbance value can be used to calculate a temperature, it is a non-linear relationship. The simple average of the absorption features along a LOS composed of several temperature values is not equal to the absorbance feature of the average temperature of this path. For this reason, absorbance must be utilized in the reconstruction process, not LOS temperature value.

Figure 4.8 shows the resulting integrated LOS temperature sinogram for the data collected on the UVaSCF for case 1 prior to background subtraction. Because these are integrated measurements, a general smearing in the temperature values is expected, i.e. the largest temperature is not the true maximum temperature within the flowfield because it has been path-averaged with regions of the flow with lower temperatures. Taking this into account, the LOS temperature sinogram agrees very well with the expected range of values. There is a clear core flow in the middle, shown in red, a transition region shown in yellow and green, and low temperature ambient room air at the edges of the fans. As mentioned previously, the output of Phase I should be the sinograms of integrated absorbance values for each of the absorption 
features, seen in Figure 4.7, since it is these integrated absorbance values that will be reconstructed in Phase II.

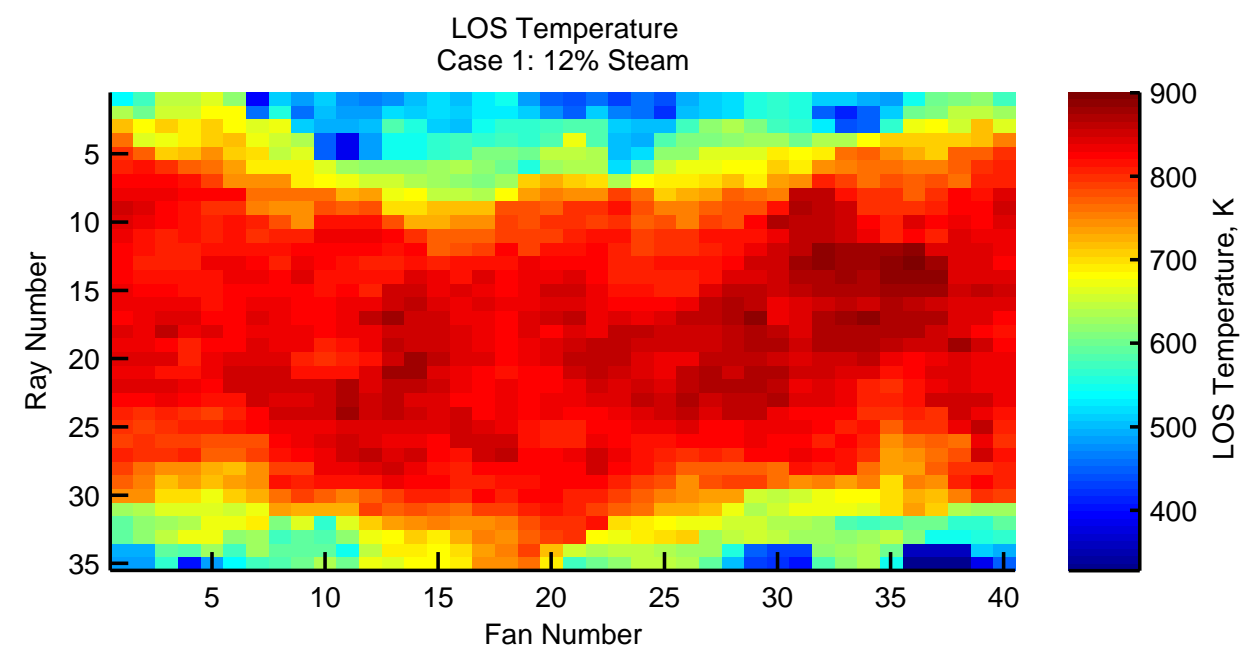

FIGURE 4.8: LOS temperatures calculated for case 1 TDLAT measurements.

Figure 4.9 shows the resulting reconstructions of integrated absorbance for all four absorbance features after background subtraction. The black rectangle superimposed on each reconstruction represents the boundary of the UVaSCF flowpath. It can be seen that the distributions of KB1 and KB2 are quite similar in shape, as predicted from their comparable sinograms. Distributions of KB2, KB3, and KB4, seen in Figures $4.9 \mathrm{~b}, 4.9 \mathrm{c}$, and $4.9 \mathrm{~d}$, each show a slight asymmetry in their distributions, namely in the bottom right corner of the distribution. 


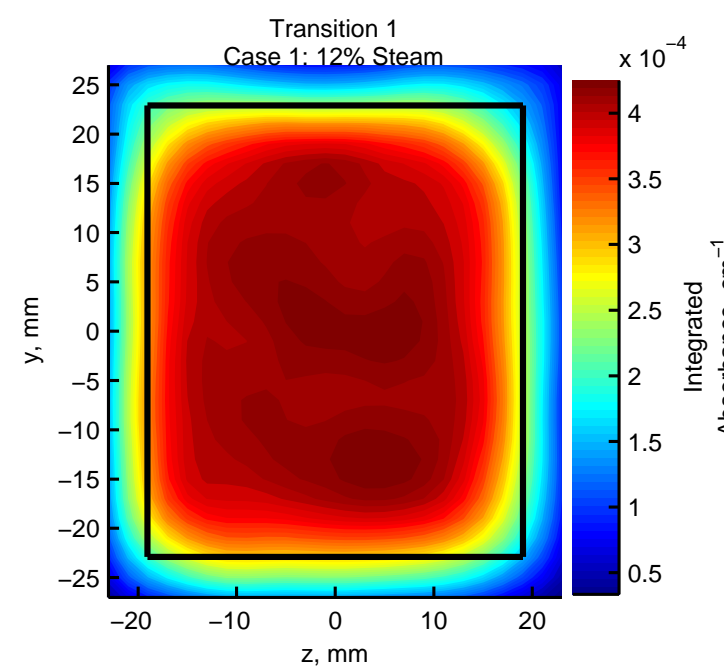

(a)

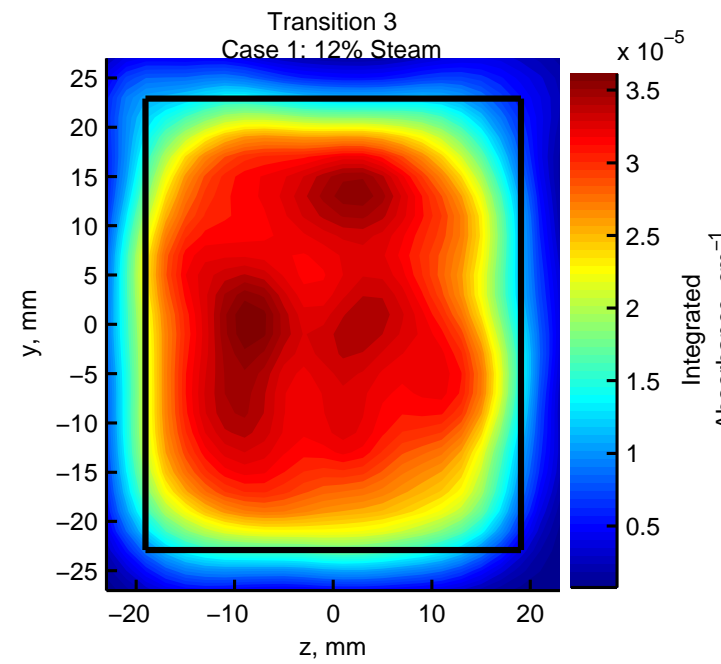

(c)

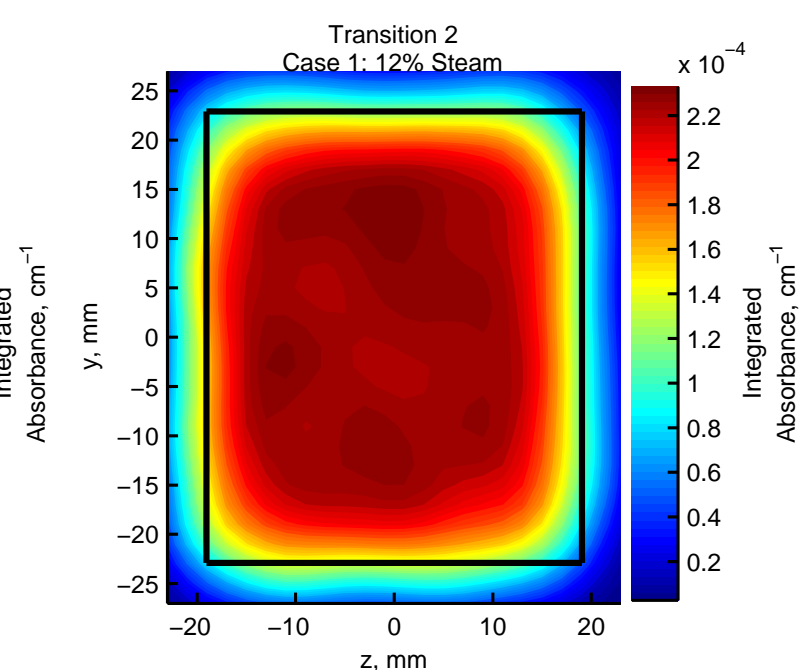

(b)

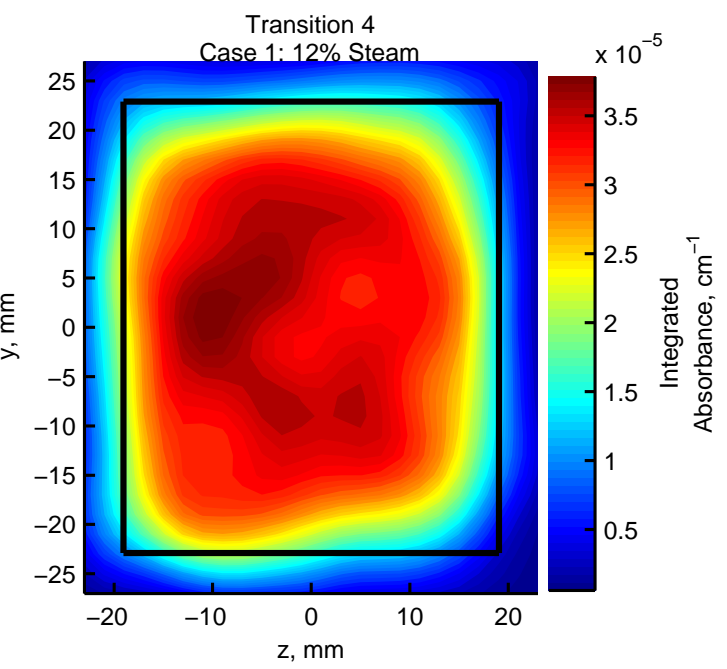

(d)

FiguRE 4.9: Reconstructed integrated absorbance at the exit plane of the UVaSCF for case 1, for (a) KB1, (b) KB2, (c) KB3, and (d) KB4. 
The temperature can be calculating using the two-line thermometry approach, as described by Equation 2.10 and is shown in Figure 4.10. Various combinations of absorption features can be utilized. As is seen in Figure 4.10, the inclusion of the KB4 absorption feature results in significantly higher temperature distributions than with the other line-pairs, and therefore will not be included in the final temperature, number density, and mole fraction calculations.

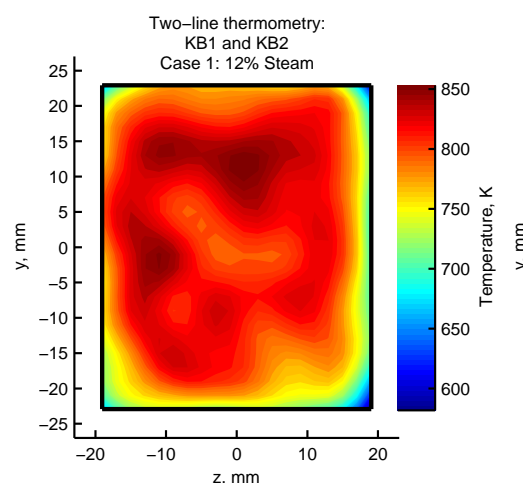

(a)

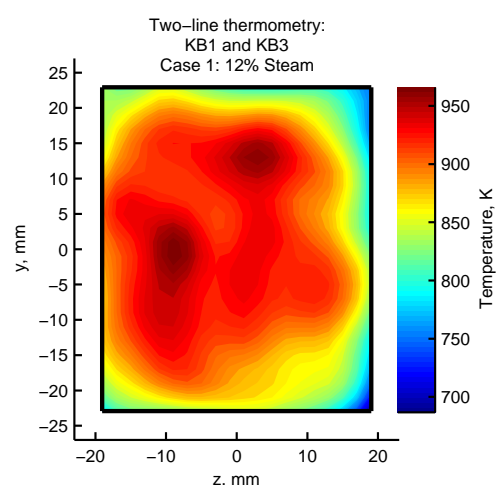

(b)

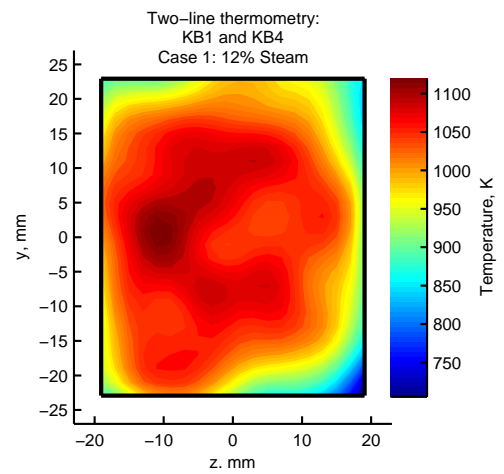

(c)

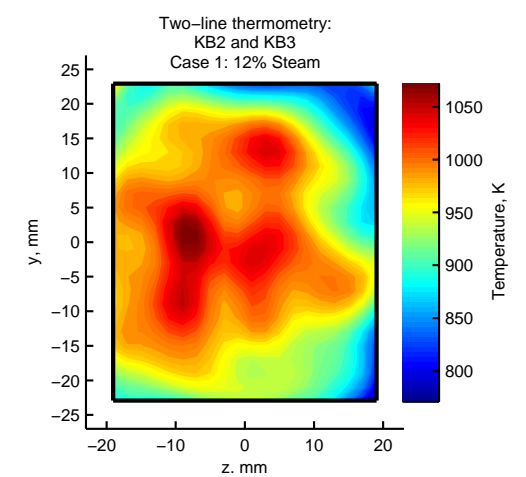

(d)

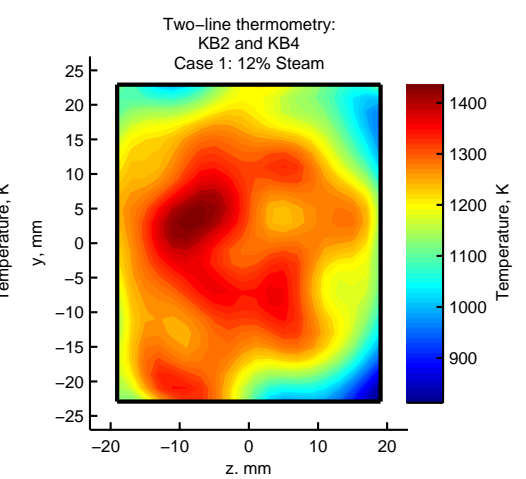

(e)

Figure 4.10: Two-line thermometry calculation via Equation 2.10 at the exit plane of the UVaSCF for case 1, for line-pairs (a) KB1 and KB2, (b) KB1 and KB3, (c) KB1 and KB4, (d) KB2 and KB3, and (e) KB2 and KB4.

Next, the Boltzmann plot analysis method was utilized as described in Equation 2.7 with absorption features KB1, KB2, and KB3. At each pixel, the temperature and number density were calculated using the reconstructed absorbance for each absorption feature. The root-mean-square error (RMSE), equal to the square root of the variance, was recorded as a measure of the goodness of the fit and has the same units as $y_{\text {Boltzmann }},\left[\ln \left(\right.\right.$ molecules $\left.\left./ \mathrm{cm}^{2}\right)\right]$. The RMSE distribution for the case 
1 Boltzmann plot results are shown in Figure 4.11.

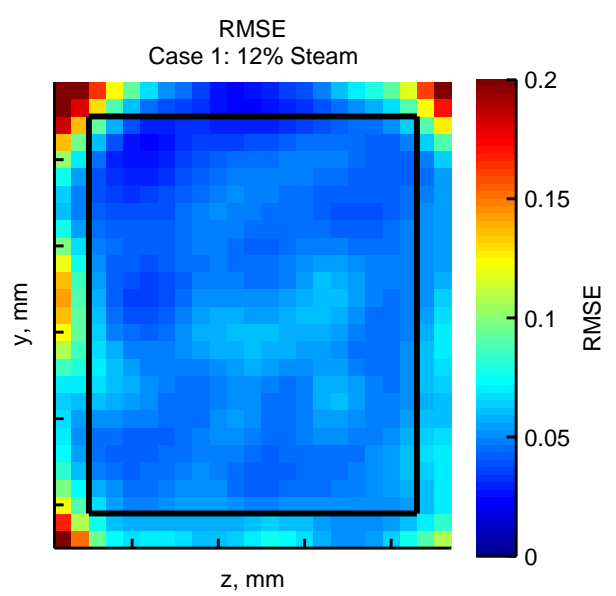

FiguRE 4.11: Root-mean square error for Boltzmann plot at the exit plane of the UVaSCF for case 1.

From the Boltzmann plot analysis, the spatially-resolved temperature, number density of water vapor, and consequently mole fraction of water vapor, were obtained and are presented in Figure 4.12. It is observed that the temperature distribution has a slight asymmetry in the z-direction. This asymmetric temperature distribution has also been observed by Cutler et al. [58] at the in-flow plane to the combustor of the UVaSCF, measured via Coherent anti-Stokes Raman Spectroscopy (CARS), and seen in Figure 4.13. For the TDLAT measurements, a maximum temperature of $996 \mathrm{~K}$ is observed, with a mean across the exit plane of $923 \mathrm{~K}$. A 1-D calculation of temperature at the UVaSCF exit plane was used to obtain an approximate static temperature range at the TDLAT measurement plane. The 1-D calculation modeled the flow from the $\mathrm{M}=2.03$ facility nozzle as an attached flow through a duct with friction, utilizing the Fanno flow equations. The range of static temperatures observed at the in-flow plane to the combustor obtained by CARS were utilized. The measured pressure rise across the extender from sub-atmospheric to atmospheric pressure was then used to calculate the rise in static temperature. The measured TDLAT temperature distribution agreed well with the 1-D calculation which resulted in a static temperature range of 925$1040 \mathrm{~K}$. The reconstructed water vapor number density shows a much more uniform distribution of water vapor molecules. Perhaps more easily relatable, the mole fraction 
of the water vapor is shown in Figure 4.12c. It is seen that the mole fraction is $12 \%$ across the majority of the duct, agreeing extremely well with the facility's $12 \%$ steam injection.

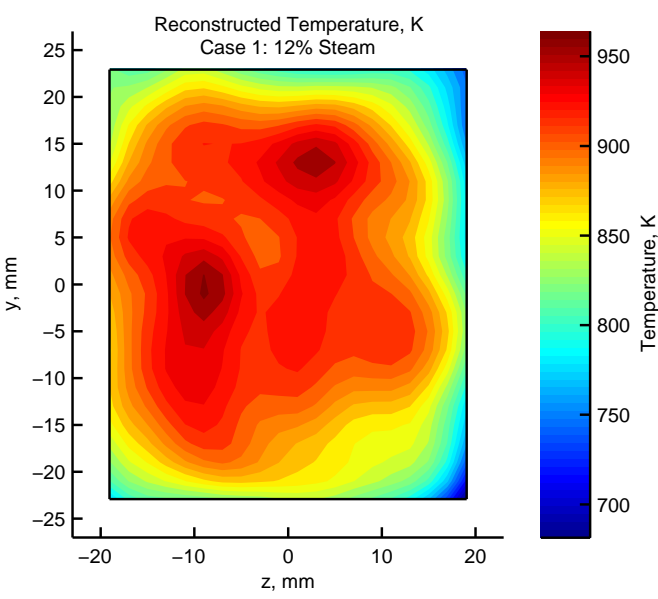

(a)

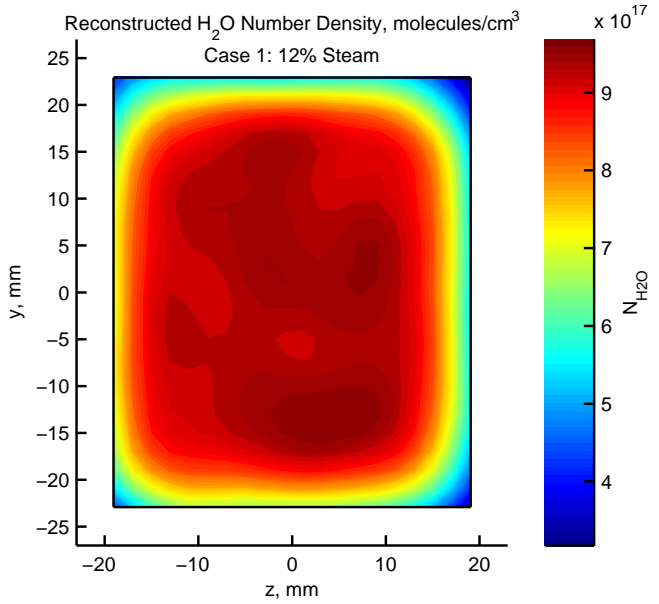

(b)

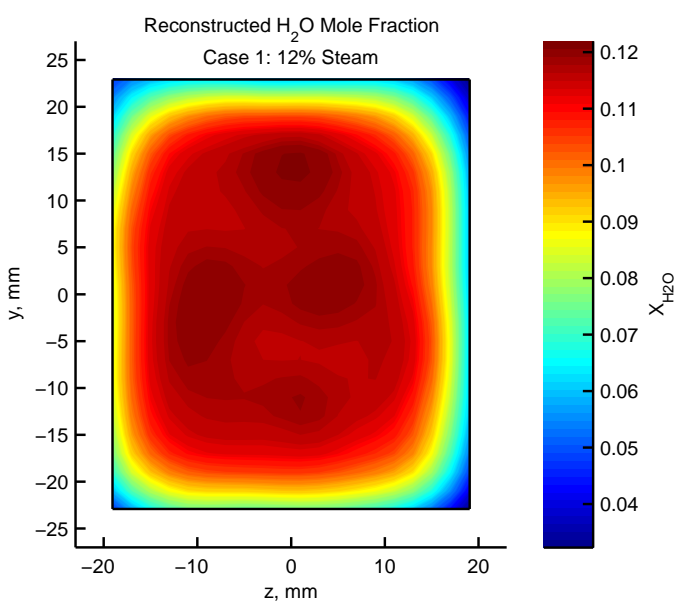

(c)

FiguRE 4.12: Final reconstructions at the exit plane of the UVaSCF for case 1: (a) static temperature, (b) water vapor number density, and (c) water vapor mole fraction. 


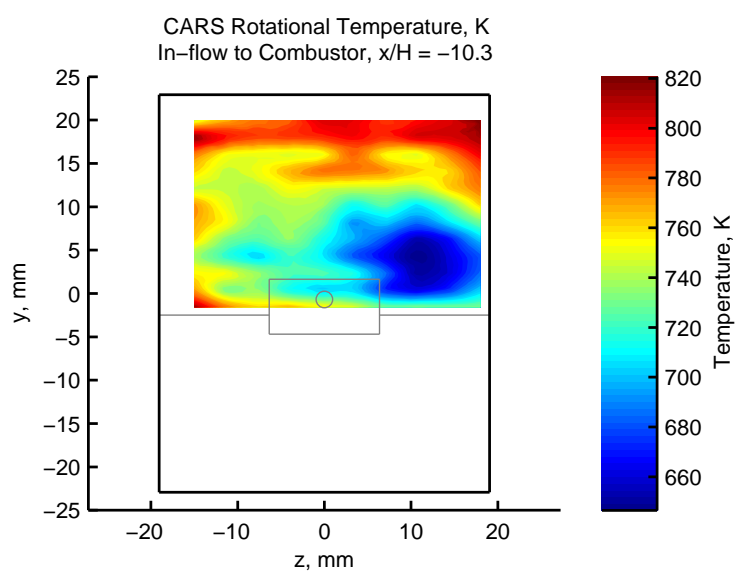

Figure 4.13: Coherent anti-Stokes Raman Spectroscopy (CARS) measurement of rotational temperature at the in-flow plane of the combustor, $\mathrm{x} / \mathrm{H}=-10.3$. [58]

Line profiles of these TDLAT measurements for case 1 are presented in Figure 4.14 and were extracted at $\mathrm{z}=0 \mathrm{~mm}$ and $\mathrm{y}=0 \mathrm{~mm}$. The temperature asymmetry is quite evident in the line-plots. Also, the uniformity of the mole fraction at $12 \%$ is confirmed. The error bars shown on each of the profiles are the uncertainties of each quantity calculated according to Section 3.4. 


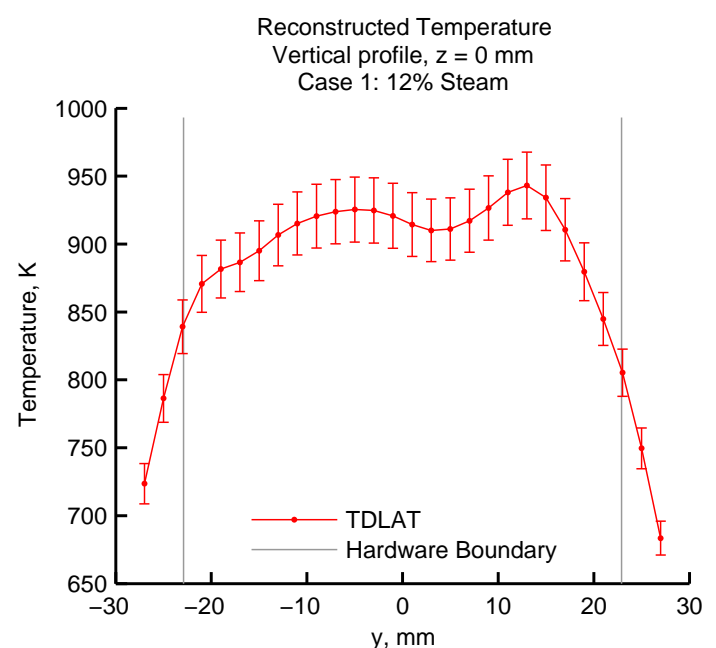

(a)

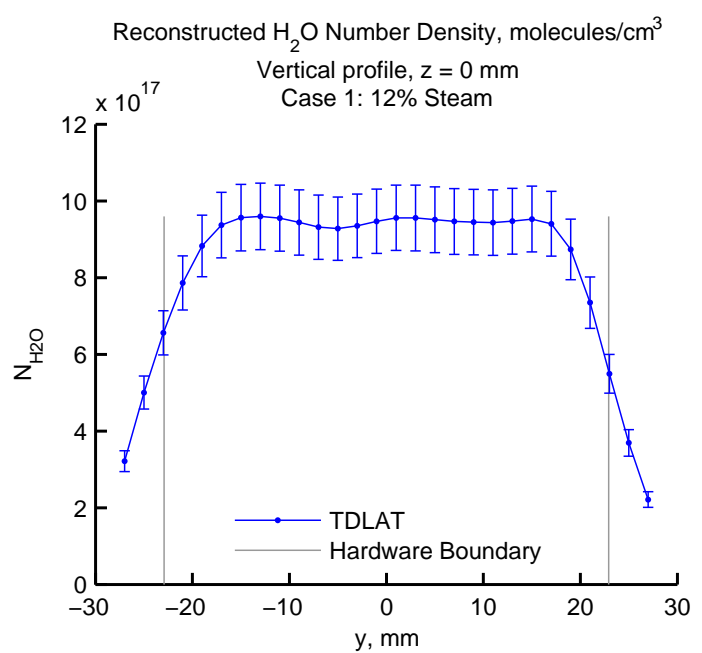

(c)

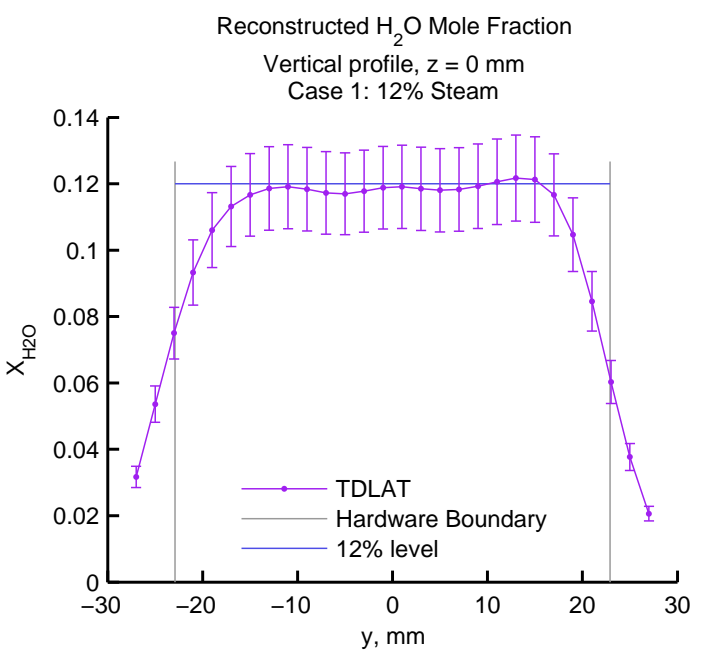

(e)

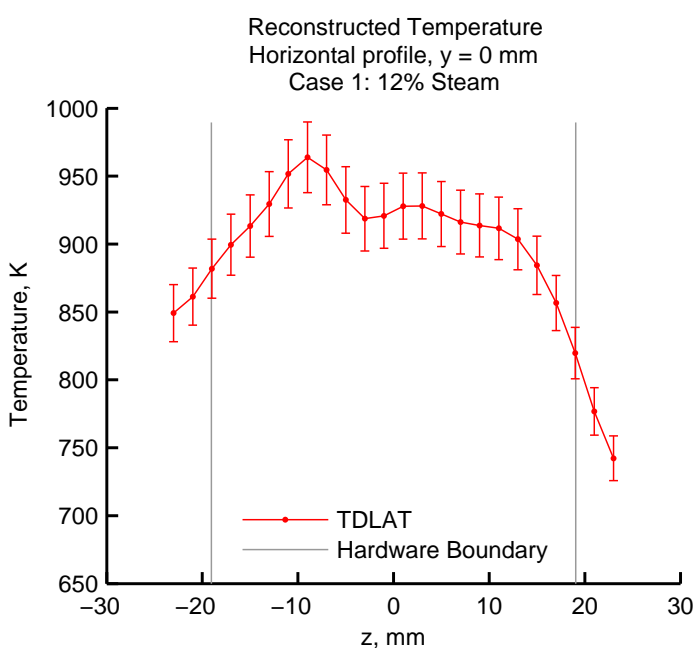

(b)

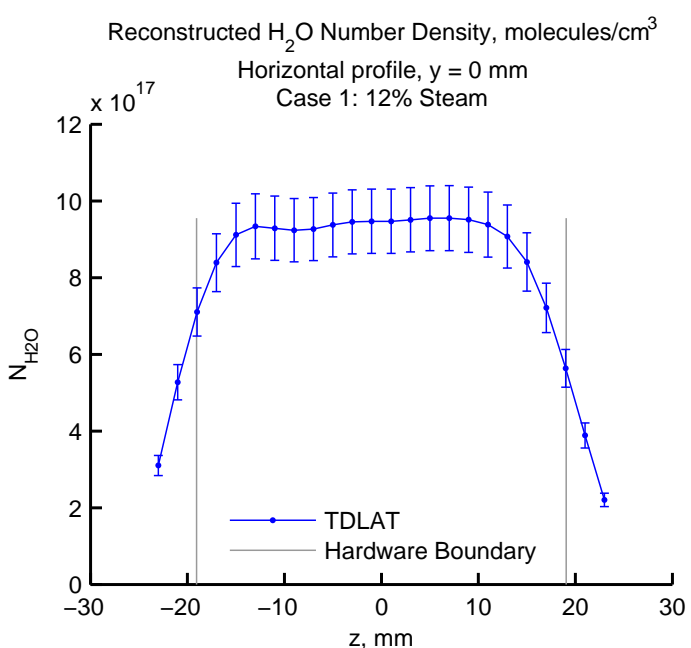

(d)

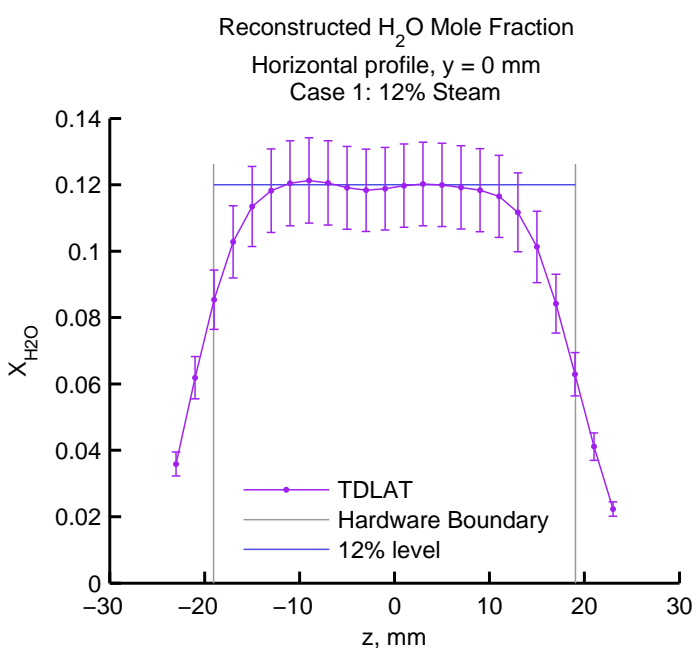

(f)

Figure 4.14: Case 1 line plots (profiles) both vertical and horizontal of (a) vertical temperature profile, (b) horizontal temperature profile, (c) vertical water vapor number density profile, (d) horizontal water vapor number density profile, (e) vertical water vapor mole fraction profile, and (f) horizontal water vapor mole fraction profile. 
Another point of comparison of these case 1 measurements is LOS TDLAS measurements performed by Goldenstein et al. [48] at the exit plane of the UVaSCF. The test section hardware varied slightly from the current configuration, but the $12 \%$ steam injection was executed identically. Goldenstein's LOS TDLAS beam originated at $\mathrm{z}=-19 \mathrm{~mm}, \mathrm{y}=0 \mathrm{~mm}$, and terminated at $\mathrm{z}=19 \mathrm{~mm}, \mathrm{y}=0 \mathrm{~mm}$, traversing the UVaSCF flowfield horizontally as presented above. For comparison, the 2-D spatially resolved water vapor mole fraction as shown in Figure 4.12c are integrated along lines of sight parallel to the direction of Goldenstein's. The path-integrated TDLAT water vapor mole fraction is shown in purple in Figure 4.15, while Goldenstein et al.'s 1 -D LOS TDLAS measurement is shown in green, at $11.5 \%, \pm 0.1 \%$ water vapor mole fraction. Along the center of the exit plane, $y=0 \mathrm{~mm}$, the path-integrated TDLAT results show excellent agreement with Goldenstein et al.'s prior measurements.

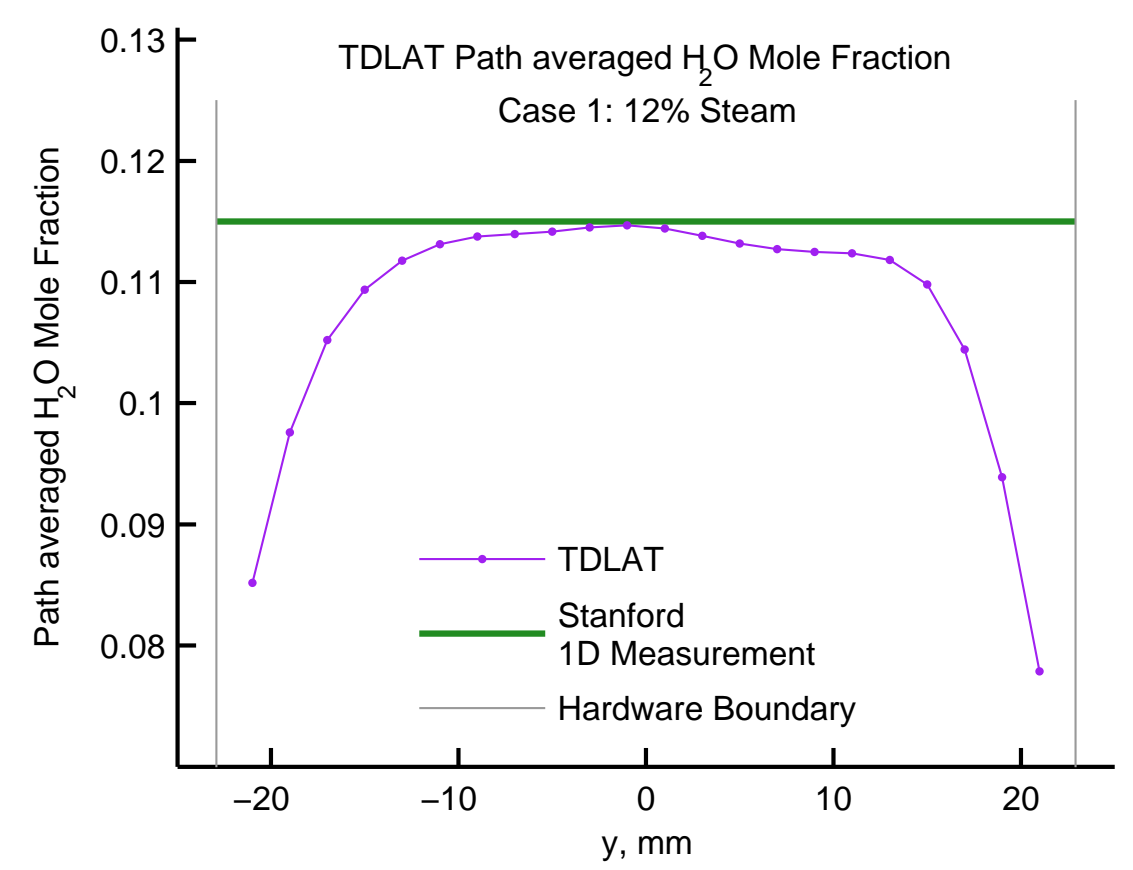

FiguRE 4.15: Path-integrated TDLAT measurement compared to 1-D Stanford Measurement at the exit plane of the UVaSCF for case 1. 
As was described in Section 3.4, 420 individual LOS measurements were collected at the same conditions for the case 1 measurements presented above. These measurements were collected along the same path as described for comparison to Goldenstein et al.'s measurements, discussed above. Figure 4.16 shows the resulting integrated absorbance values for the three absorption features over 420 LOS.

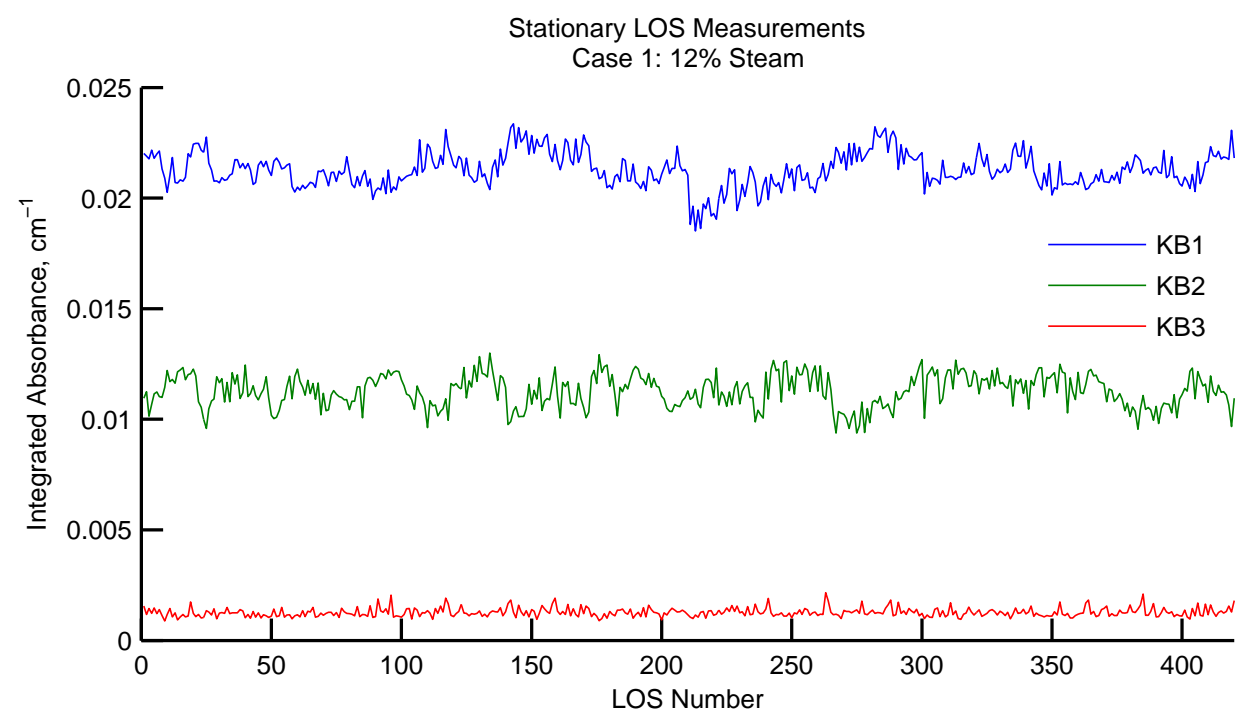

FIGURE 4.16: 420 stationary LOS collected at case 1 conditions.

From these stationary LOS measurements, the mean, standard deviation, and \% noise of the integrated absorbance for absorption features KB1, KB2, and KB3 can be determined and are tabulated in Table 4.2 .

TABLE 4.2: Statistical properties of case 1 measurements.

\begin{tabular}{llll}
\hline Absorption Feature & $\begin{array}{l}\text { Mean, } \mathrm{cm}^{-1} \\
\bar{A}\end{array}$ & $\begin{array}{l}\text { Standard Deviation, } \mathrm{cm}^{-1} \\
\sigma_{L O S}\end{array}$ & $\begin{array}{l}\% \text { Noise } \\
\sigma_{L O S} / \bar{A}\end{array}$ \\
\hline KB1 & .021268 & .00078483 & $3.69 \%$ \\
KB2 & .011273 & .00073611 & $6.53 \%$ \\
KB3 & .021268 & .00020226 & $15.97 \%$ \\
\hline
\end{tabular}

Following the procedure outlined in Section 3.4, the uncertainties and \% uncertainties (the uncertainty compared to the actual values) for the case 1 measurements shown in Figure 4.12 are shown in Figure 4.17. 


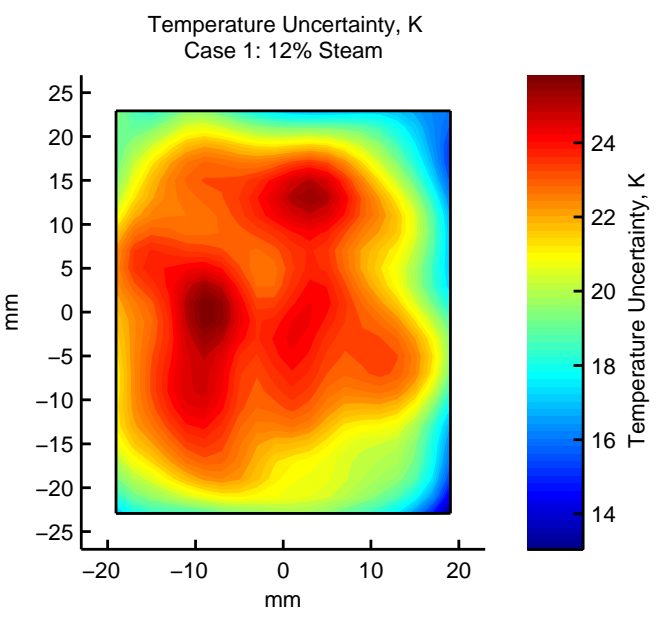

(a)

Reconstructed $\mathrm{H}_{2} \mathrm{O}$ Number Density Uncertainty, molecules $/ \mathrm{cm}^{3}$

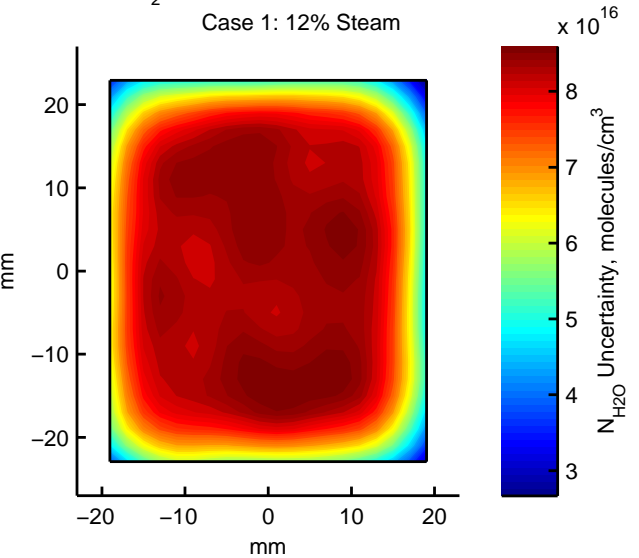

(c)

Reconstructed $\mathrm{H}_{2} \mathrm{O}$ Mole Fraction Uncertainty

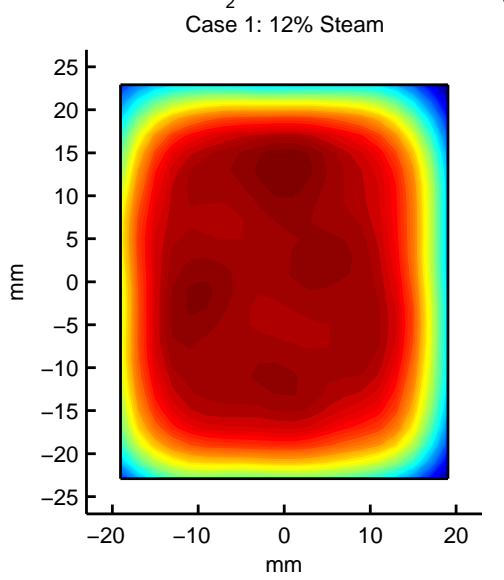

(e)
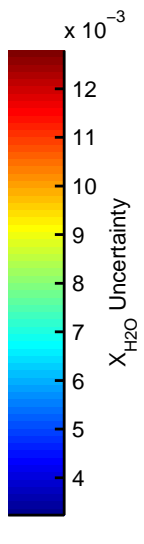

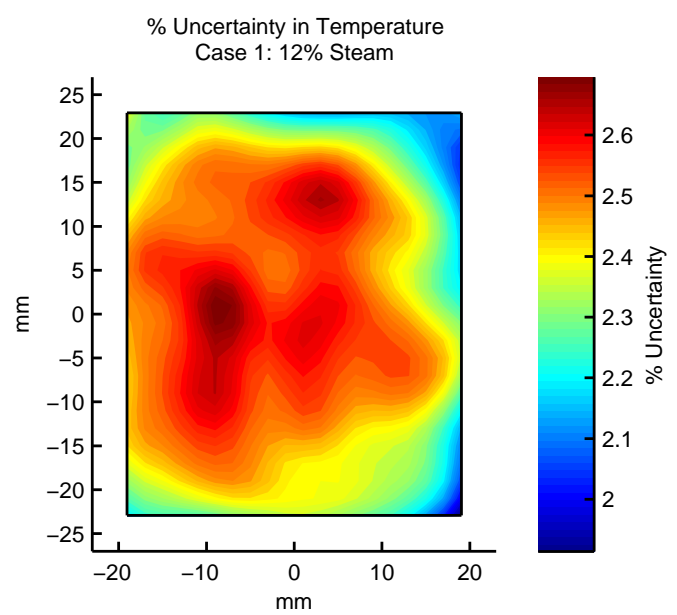

(b)

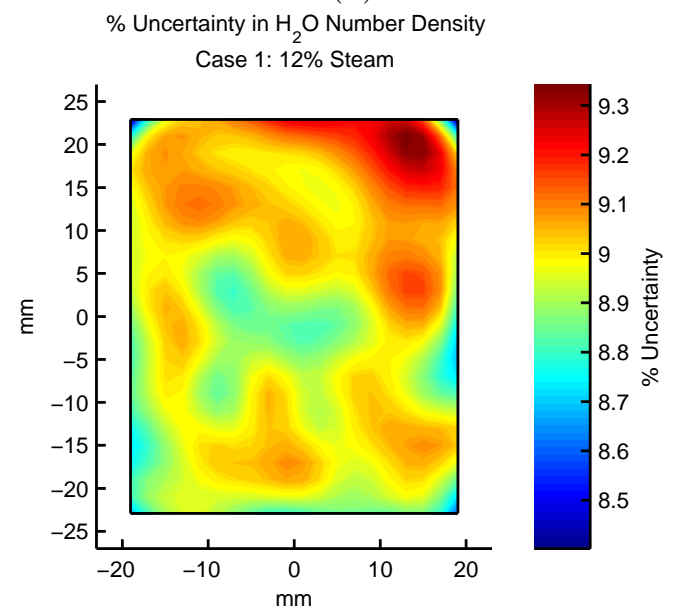

(d)

\% Uncertainty in $\mathrm{H}_{2} \mathrm{O}$ Mole Fraction

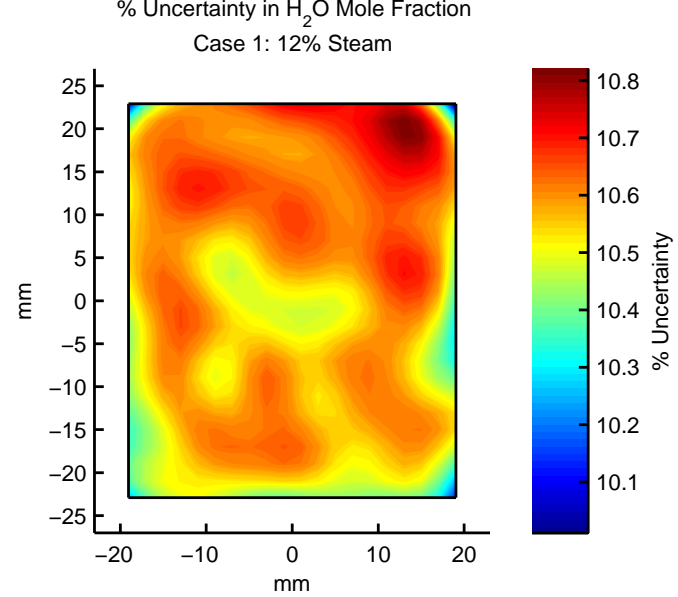

(f)

FiguRE 4.17: Absolute uncertainty and $\%$ of full value uncertainty for case 1 reconstructions of temperature, water vapor number density, and water vapor mole fraction. 


\subsection{Case 2: Scram-mode $\phi=0.18$ Results}

The next conditions measured by TDLAT on the UVaSCF are for a relatively low fuel-to-air equivalence ratio, $\phi$, where $\phi$ is the ratio of the actual fuel-to-air ratio to the stoichiometric fuel-to-air ratio. The TDLAT measurements were collected with $\phi=0.18$, simulating a scram-mode of combustion. At this equivalence ratio, there is no pre-combustion shock train, resulting in supersonic flow across the entire duct entering the combustor. Table 4.1 shows the test conditions for data collected in case 2 on August 23, 2013.

The measured sinograms of integrated absorbance for all four absorbance features for case 2 are shown in Figure 4.18, again with the raw sinograms in the first column, and the filtered sinograms in the second column, prior to background subtraction. KB1's sinogram shows more noise during this combusting mode of operation than it did for the case 1 conditions. An asymmetry is seen in both the raw and filtered KB1 sinograms, Figures 4.18a and 4.18b. This asymmetry has been observed for all analysis conducted for the $\phi=0.18$ measurements, including analysis of this data set by modeling the absorbance profile by the modified Whiting expression (discussed in Section 2.4.1) and Abrarov's exponential series approximation, as well as for a set of TDLAT measurements acquired on a separate measurement campaign with a different TDLAT fanbeam geometry $\left(\Delta_{\alpha}, \alpha, N_{\text {rays }}\right.$ different than the presented measurements). The KB2 sinogram, however, shows great symmetry and signal-to-noise ratio (SNR), even in the raw sinogram. The KB3 and KB4 sinograms have a reasonable level of SNR, but are both significantly improved with minimal filtering.

As with the measurements obtained for case 1, the integrated absorbance sinograms of case 2 can be used to calculate a sinogram of integrated LOS temperatures. Though not for reconstruction, this straight-forward calculation allows for preliminary analysis of the measured data. Seen in Figure 4.19, the LOS temperatures are slightly higher than were observed in case 1, with a significantly higher amount of structure seen in the sinogram. Both of these observations are in agreement with the 


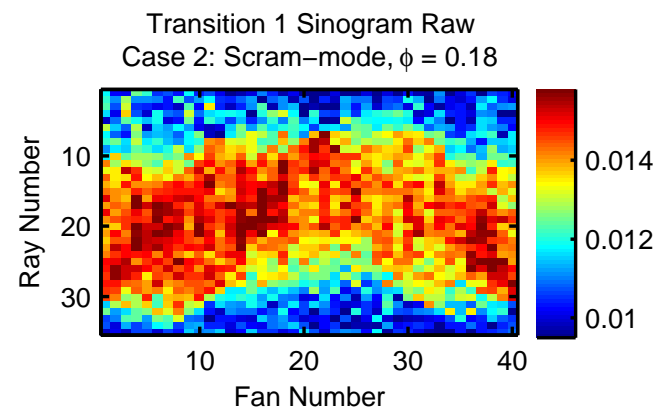

(a)

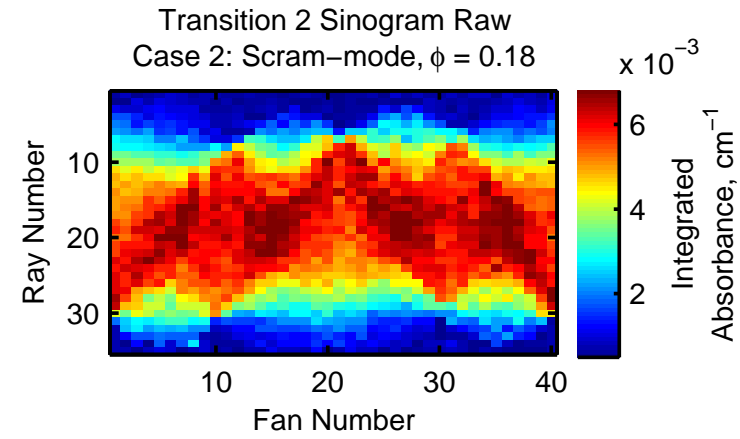

(c)

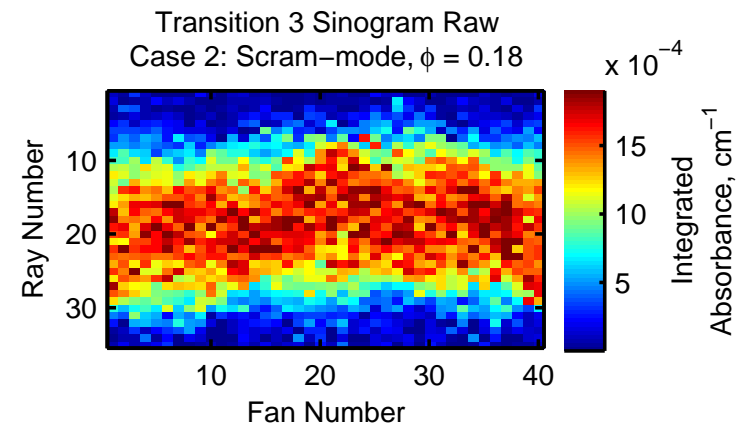

(e)

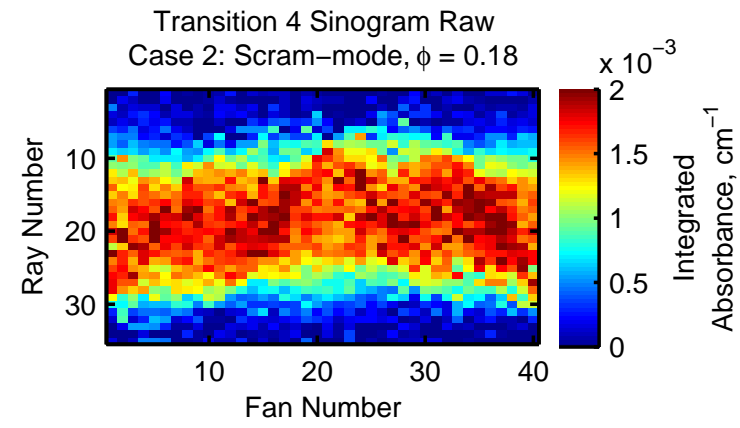

$(\mathrm{g})$

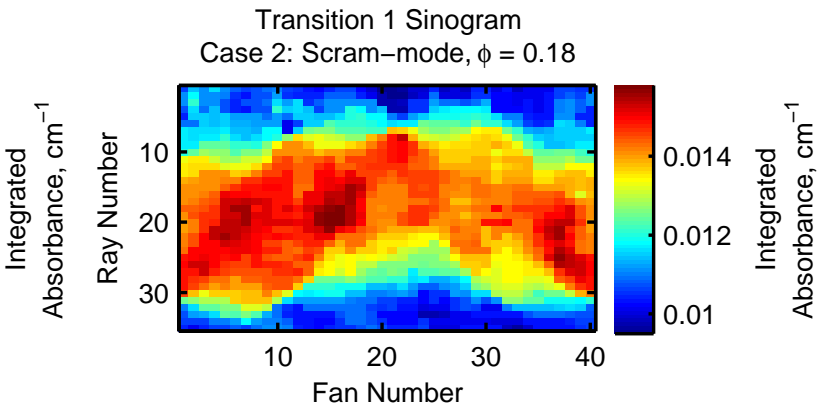

(b)

Transition 2 Sinogram

Case 2: Scram-mode, $\phi=0.18 \quad \times 10^{-3}$

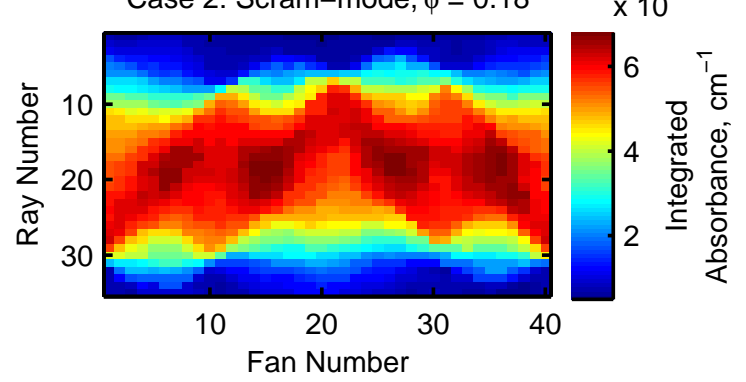

(d)

Transition 3 Sinogram

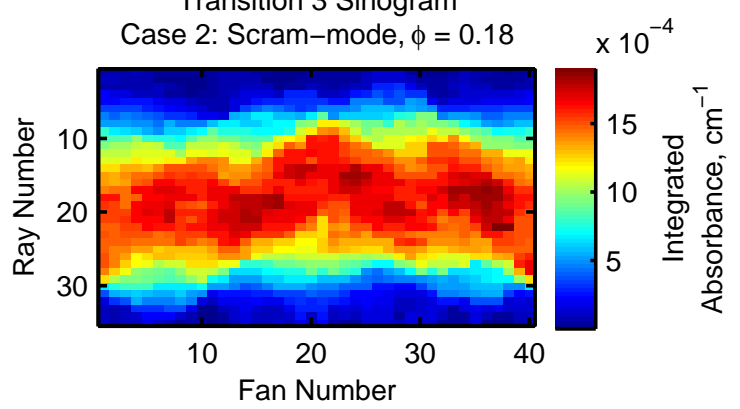

(f)

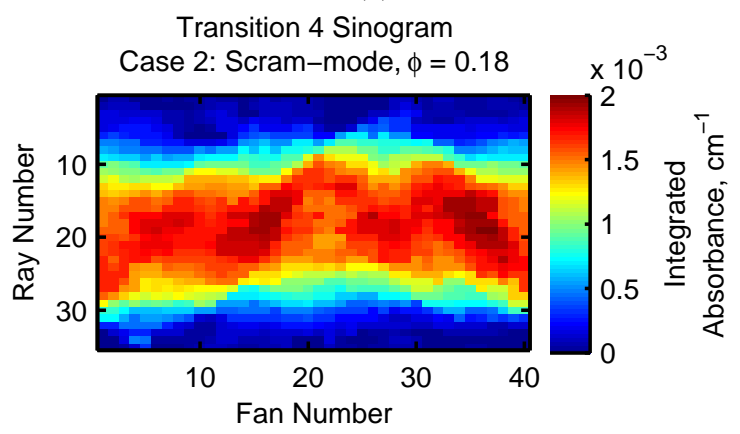

(h)

FiguRE 4.18: Integrated absorbance sinograms at the exit plane of the UVaSCF for case 2. The first column contains the raw sinograms of TDLAT measured integrated absorbance for each of the four absorption features (KB1-KB4) and the second column contains the corresponding filtered sinograms. 
expected characteristics of the temperature distribution of case 2 .

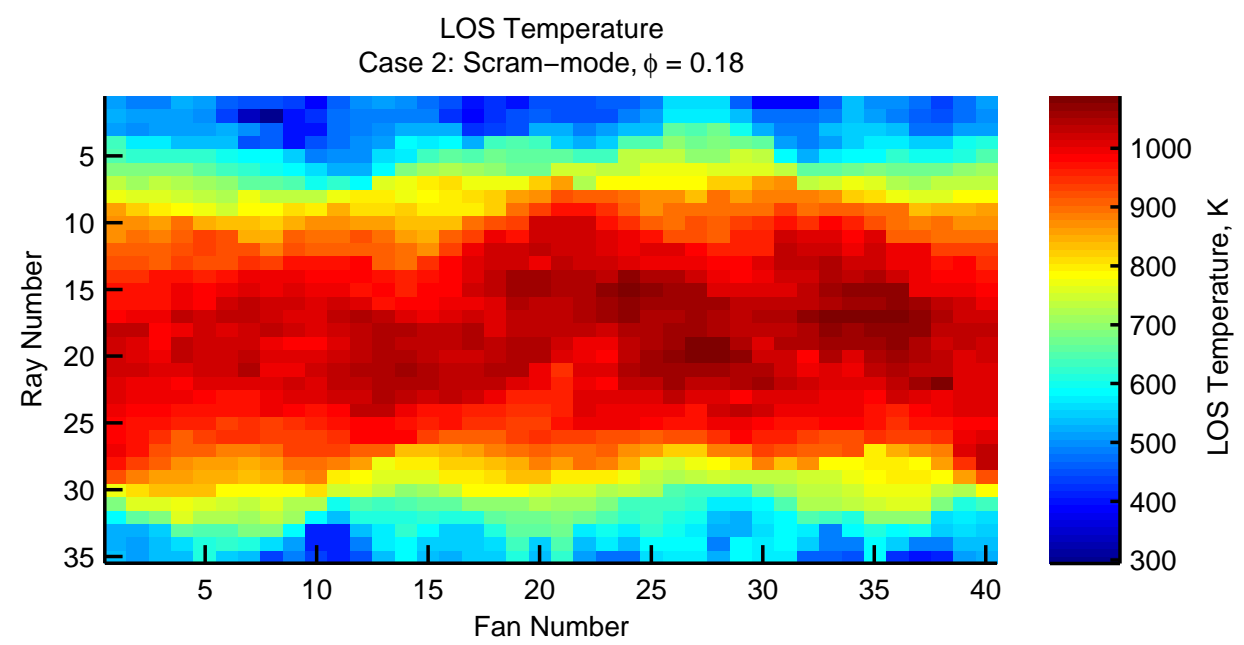

Figure 4.19: LOS temperatures calculated for case 2 TDLAT measurements.

The background absorbance was then subtracted from the measured data shown in Figure 4.18. These sinograms were then reconstructed using the ML-EM algorithm, resulting in four distributions of integrated absorbance and presented in Figure 4.20. Unlike what was seen in case 1, the sinograms of KB1 and KB2 do not look exceedingly similar, and this is reiterated by their differing reconstructed absorbances, seen in Figures $4.20 \mathrm{a}$ and $4.20 \mathrm{~b}$.

The two-line thermometry distributions are seen in Figure 4.21 for various combinations of the four absorption features. Again as before, Phase I and II analysis for the KB4 absorption feature has been carried out, but will not be included in Phase III analysis until its spectral parameters can be confirmed. 


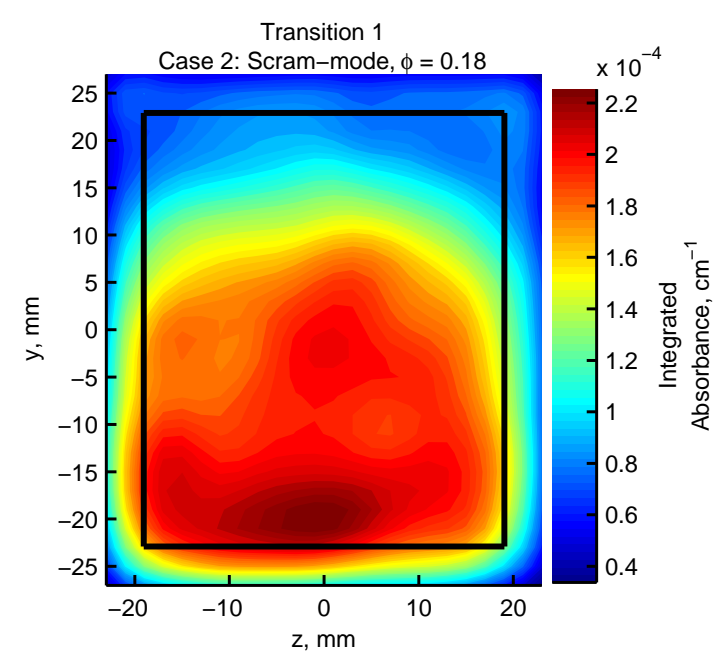

(a)

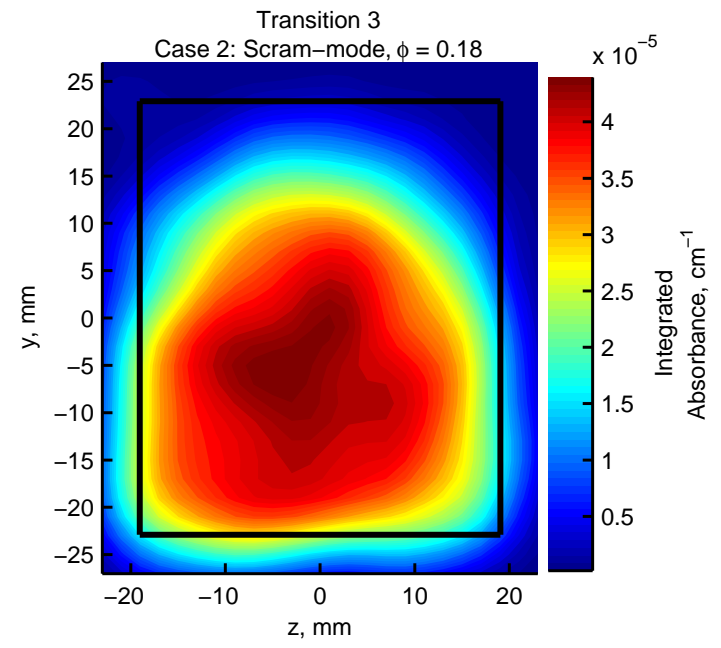

(c)

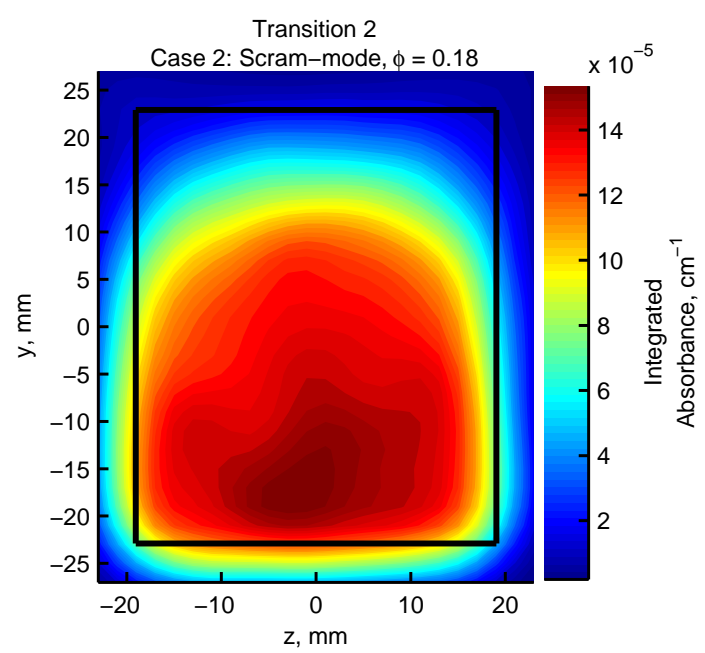

(b)

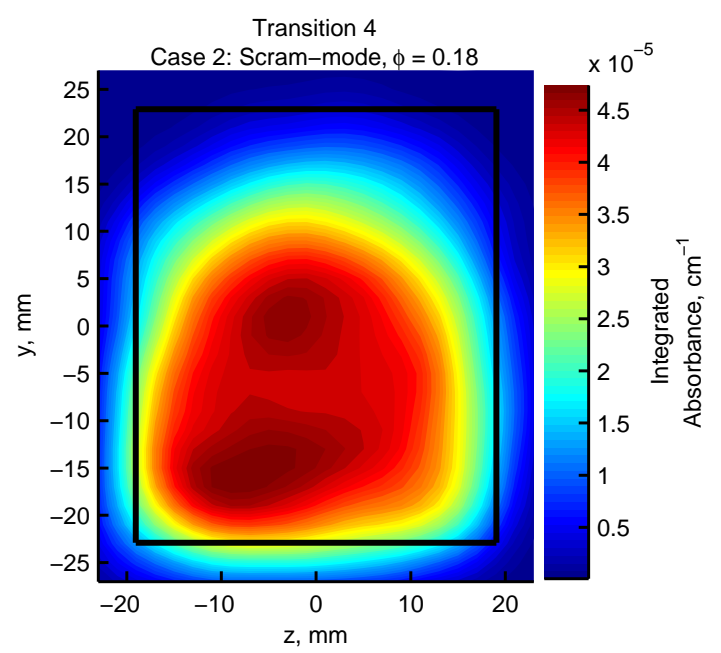

(d)

FigURE 4.20: Reconstructed integrated absorbance at the exit plane of the UVaSCF for case 2, for (a) KB1, (b) KB2, (c) KB3, and (d) KB4. 


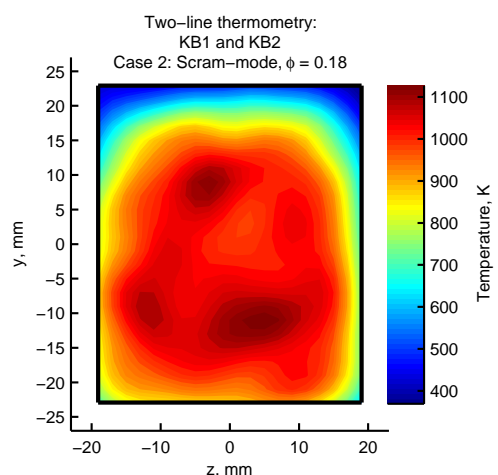

(a)

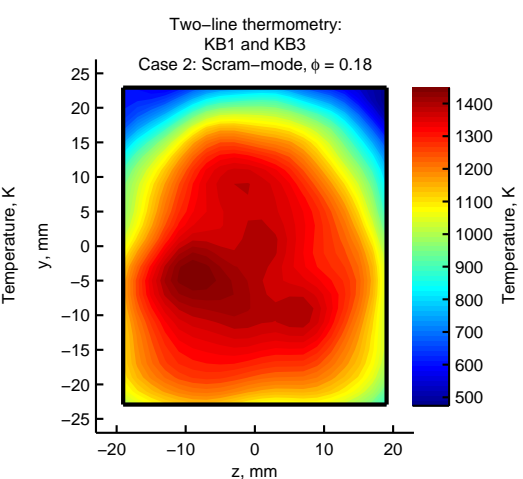

(b)

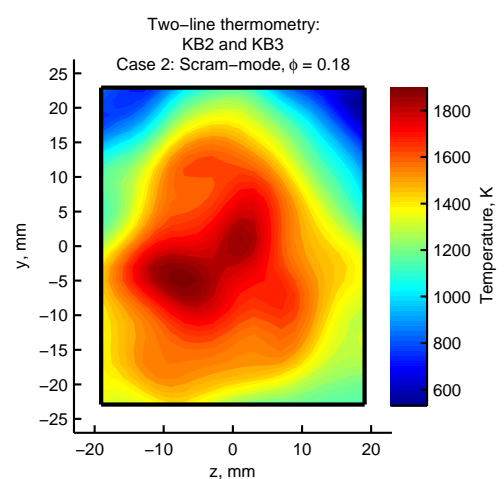

(c)

Figure 4.21: Two-line thermometry calculation via Equation 2.10 at the exit plane of the UVaSCF for case 2 for line-pairs (a) KB1 and KB2, (b) KB1 and KB3, and (c) KB2 and KB3.

The Boltzmann plot analysis was carried out for the case 2 measurements and the RMSE of the non-linear least-squares fit is shown in Figure 4.22. Again, extremely high-quality fitting of the parameters is observed within the main region of interest (Region A), with more error observed in pixels outside of this core area defined by the black rectangle.

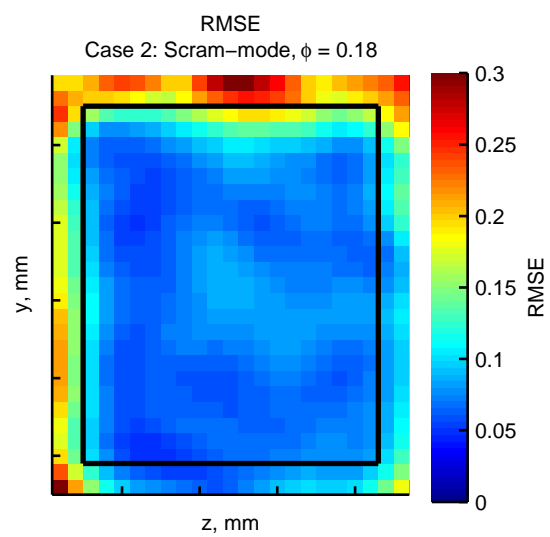

Figure 4.22: Root-mean square error for Boltzmann plot at the exit plane of the UVaSCF for case 2 .

Figure 4.23 shows the final temperature, water vapor number density, and water vapor mole fraction distributions for the scram-mode case 2 TDLAT measurements via Boltzmann plot analysis. The shapes of these distributions differ significantly from the distributions seen in Figure 4.12 for case 1, as was expected. Higher temperatures are 
seen, with maximum values near 1,400 K, and approaching ambient conditions toward the boundaries of the UVaSCF exit plane hardware. Again, a slight asymmetry in the temperature distribution is observed. The temperature and water vapor concentration are seen to be the highest toward the fuel-injector wall, suggesting that the flame is anchored to the wall and that the combustion completeness is likely very high in this region of the flow. The water vapor number density and water vapor mole fraction show similar trends as the temperature, but do not show the same asymmetry in the z-direction.

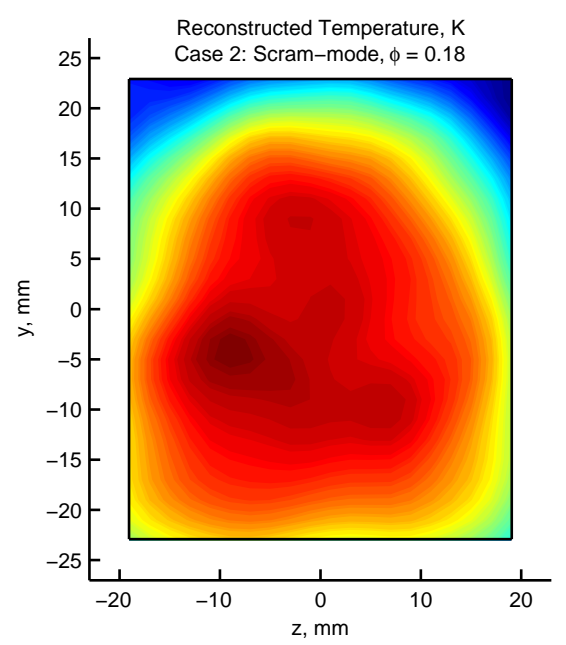

(a)
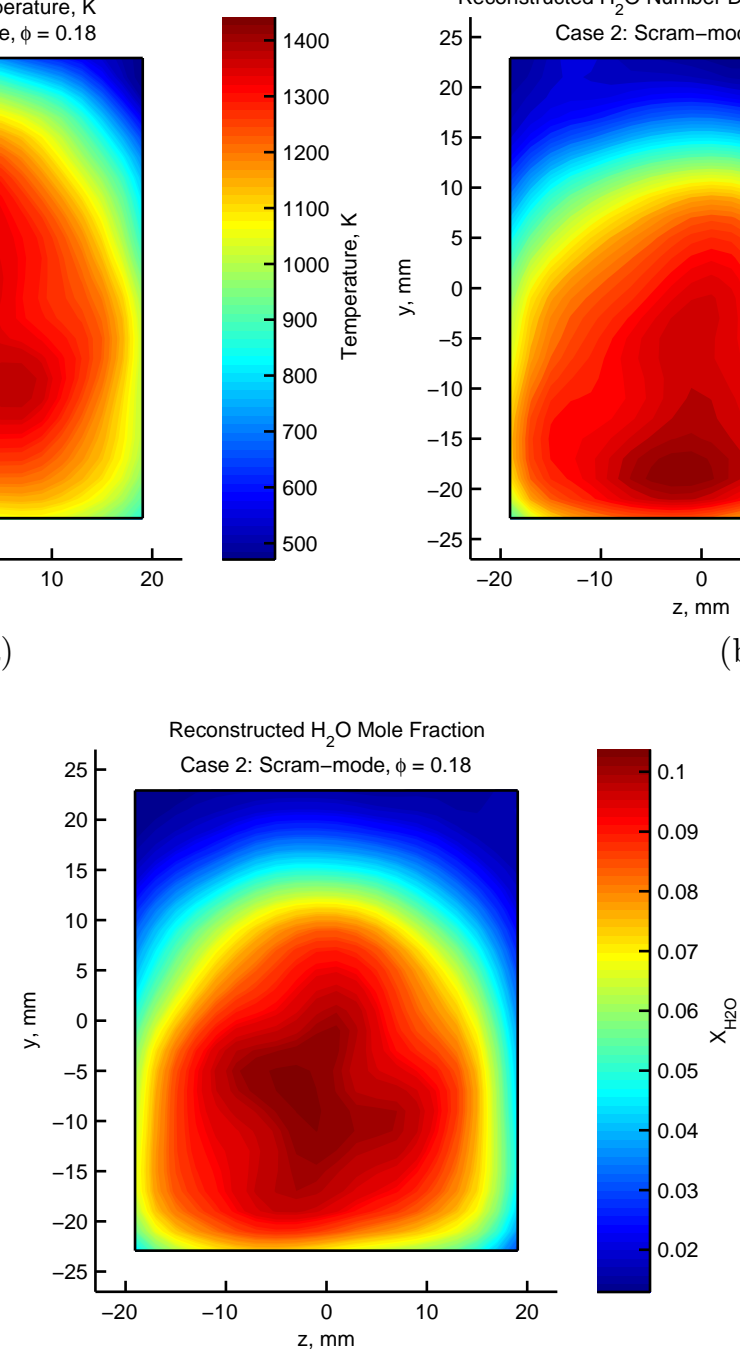

(c)

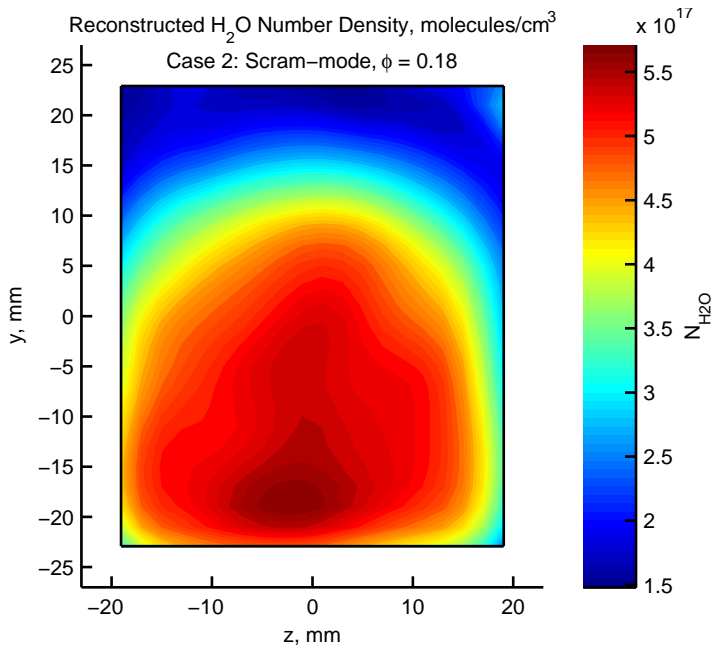

(b) 
As was a major motivation for this work, the TDLAT results for case 2 can be directly compared to computational fluid dynamic (CFD) calculations performed by Edwards and Fulton at N.C. State University in support of the NCHCCP [55]. Figure 4.24 shows side-by-side comparisons of the temperature, water vapor number density, and water vapor mole fraction as measured by TDLAT and simulated by CFD. These comparisons are plotted on the same color scales, to highlight both areas of good agreement and disagreement. It is readily apparent that the CFD predicts higher temperatures than are observed by the TDLAT measurements. However, the shape of the distributions agree quite well, with the hottest core of the flow sitting near the fuel-injector wall $(\mathrm{y}=-23 \mathrm{~mm})$ and similar penetration of this hot zone across the duct. The magnitudes of the water vapor number density calculated by CFD and measured by TDLAT are in excellent agreement. The CFD over-predicts the water vapor number density near the fuel-injector wall and in the lower two corners, but agree extremely well in the remainder of the duct, both in magnitude and distribution. As mole fraction is a quantity derived from both temperature and number density, it is not surprising that the CFD calculated water vapor mole fraction also shows a significant difference in magnitude from the measured quantity, following the trend seen in the temperature comparisons. Again, while the magnitude of the two water vapor mole fraction distributions differ, the overall shape and penetration of the water vapor into the duct are very similar.

To better understand the similarities and discrepancies between the CFD calculations and TDLAT measurements for case 2, line plots of all three quantities (temperature, water vapor number density, and water vapor mole fraction) for both CFD and TDLAT are shown in Figure 4.25. The left column shows vertical profiles taken at $\mathrm{z}=0 \mathrm{~mm}$, while the right column shows horizontal profiles taken at $\mathrm{y}=0 \mathrm{~mm}$. The CFD calculations are plotted as a thin black line, while the TDLAT measurements are shown in color. 


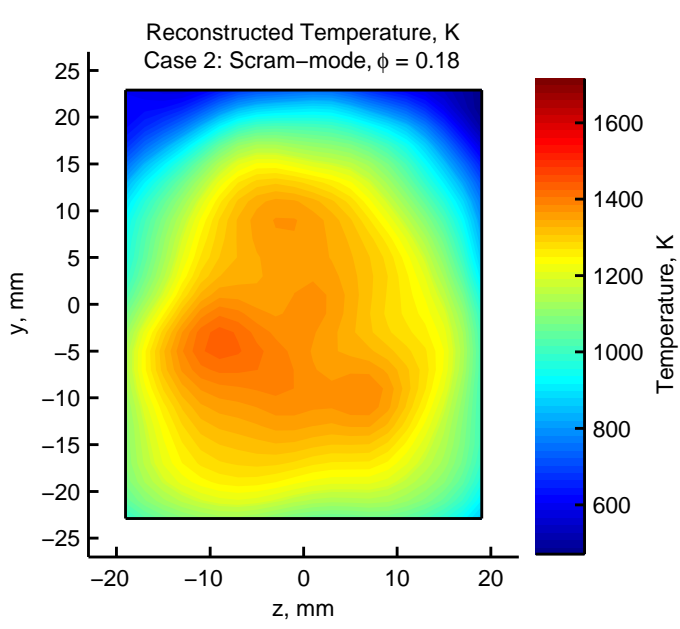

(a)

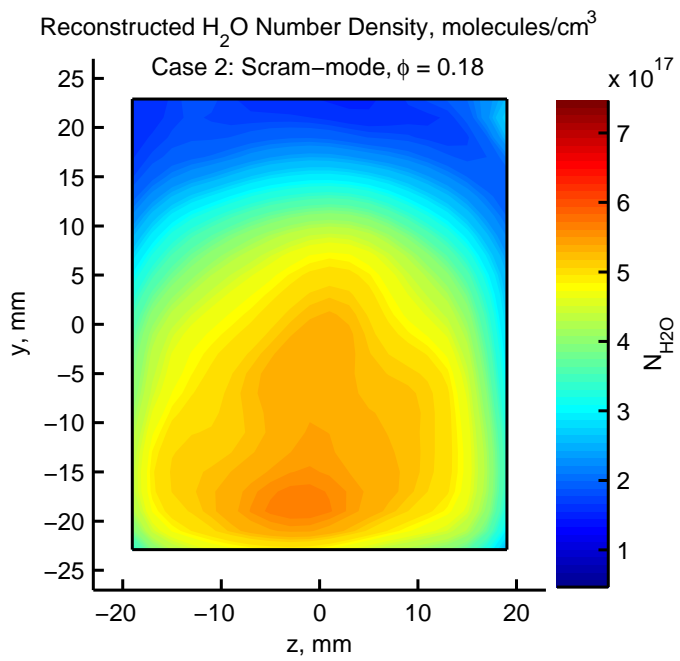

(c)

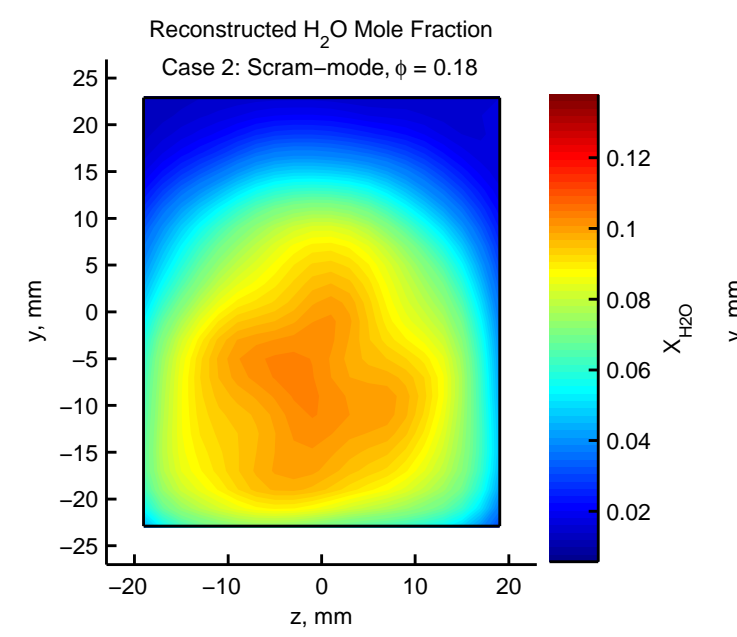

(e)

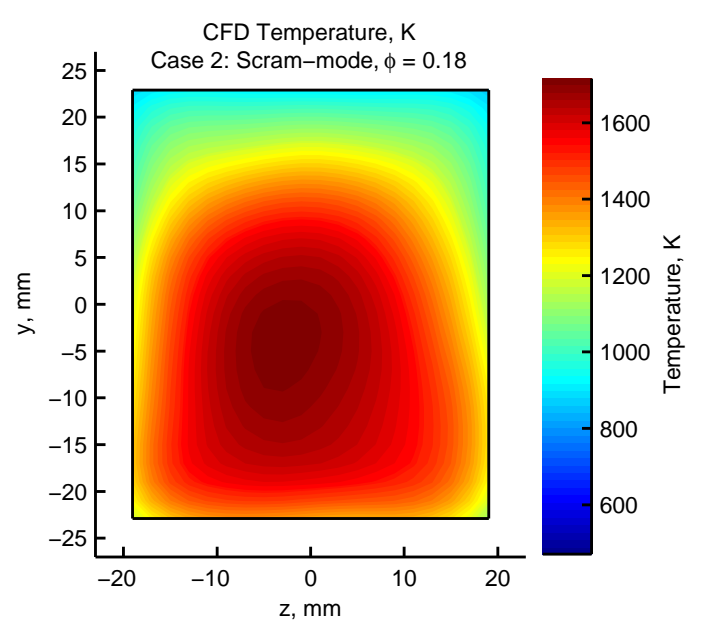

(b)

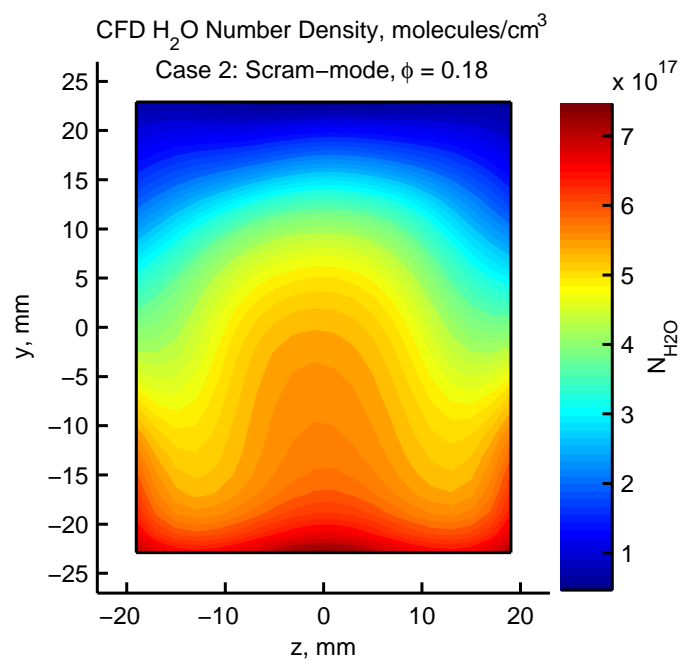

(d)

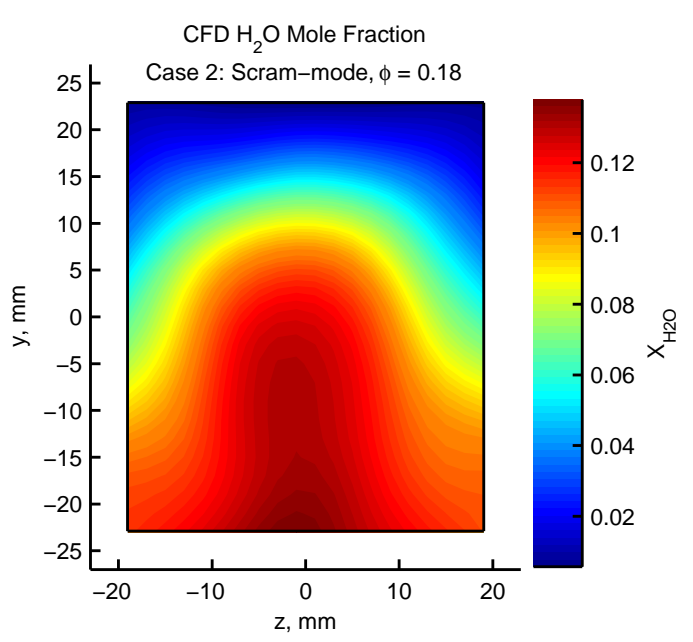

(f)

FiguRE 4.24: Side-by-side comparisons of TDLAT measurements and CFD at the exit plane of the UVaSCF for case 2, (a) TDLAT temperature distribution, (b) CFD temperature distribution, (c) TDLAT water vapor number density distribution, (d) CFD water vapor number density distribution, (e) TDLAT water vapor mole fraction distribution, and (f) CFD water vapor mole fraction distribution. 


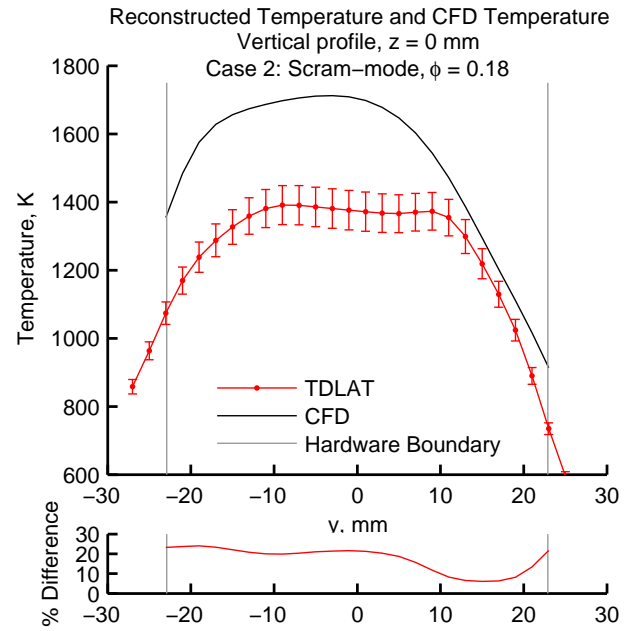

(a)

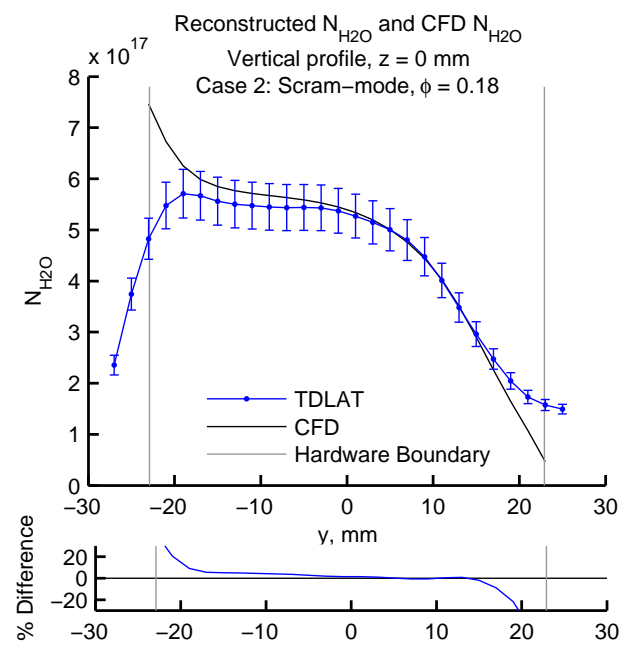

(c)

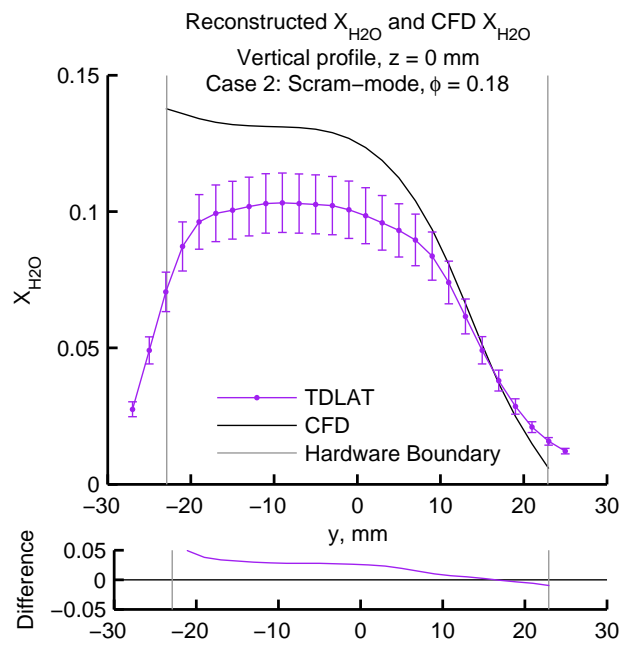

(e)

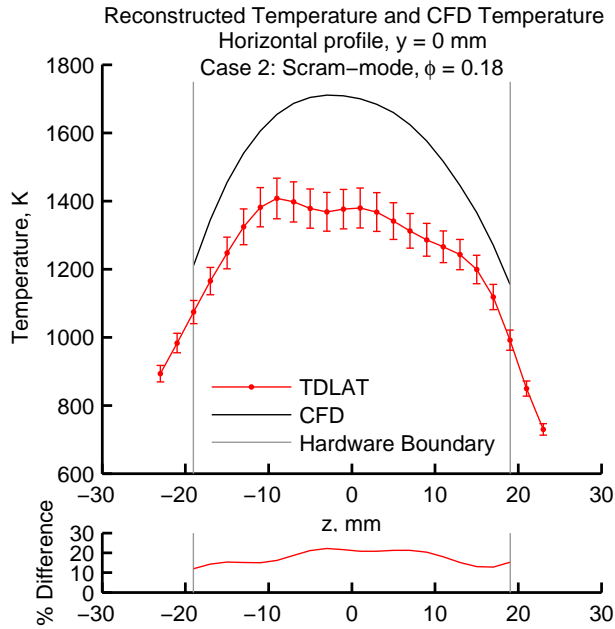

(b)

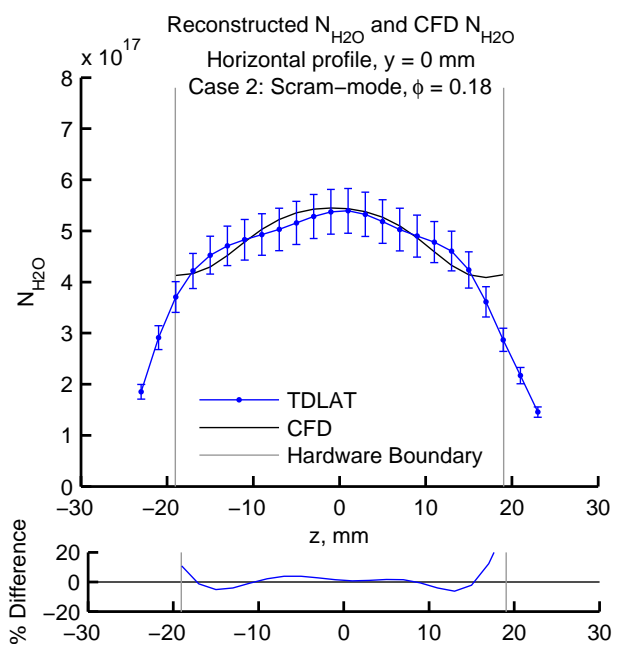

(d)

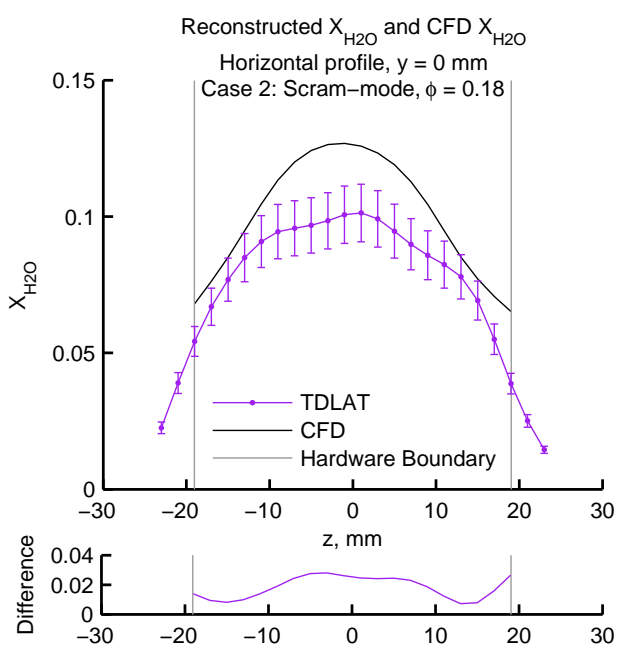

(f)

FiguRE 4.25: Line plot comparisons of TDLAT measurements and CFD at the exit plane of the UVaSCF for case 2, (a) vertical profiles for TDLAT and CFD temperatures, (b) horizontal profiles for TDLAT and CFD temperatures, (c) vertical profiles for TDLAT and CFD water vapor number densities, (d) horizontal profiles for TDLAT and CFD water vapor number densities, (e) vertical profiles for TDLAT and CFD water vapor mole fractions, and (f) horizontal profiles for TDLAT and CFD water vapor mole fractions. 
The first row of Figure 4.25 compares the static temperature distributions. The discrepancy in temperature is more easily seen in this plot, differing by a maximum of $300 \mathrm{~K}$ at some points in the flow. This disagreement between experimentallymeasured and CFD-calculated temperatures has been observed in comparisons of the CFD to other experimental measurements. When compared to Coherent anti-Stokes Raman Spectroscopy (CARS) measurements for the same UVaSCF conditions, the CFD was observed to over-predict the measured flame temperatures by several hundred degrees $\mathrm{K}$ at two planes downstream of the point of fuel injection. [55] According to Fulton et al., the higher temperatures are a result of the selected kinetics model by Burke et al. [59] This newer model is more reactive than their previously utilized mechanism, the Jachimowski model. This exaggerated amount of heat release by the utilized kinetics model both raises the flame temperature and improves the capability of keeping the flame "lit" in the simulation. [55] Though this discrepancy in temperature between the CFD simulation and CARS measurements seems to diminish as the axial distance from the fuel injector increases, it is certainly feasible that the discrepancy in temperature between the CFD simulation and the TDLAT measurements could stem from the same source.

The second row of Figure 4.25 compares the simulated and measured TDLAT water vapor number densities. These line plots confirm the exceptional agreement between the simulation and TDLAT measurements of the water vapor number density distribution for case 2. From Figure $4.25 \mathrm{c}$, it is observed that the measured penetration of the water vapor across the duct is nearly exactly as predicted by CFD, especially between $\mathrm{y}=-15 \mathrm{~mm}$ and $\mathrm{y}=15 \mathrm{~mm}$, where the $\%$ difference between the two profiles is less than $5 \%$. It can be seen from this vertical profile that the largest discrepancy between the CFD water vapor number density and the measured TDLAT water vapor number density is found along $\mathrm{y}=-23 \mathrm{~mm}$, along the vertical gray line representing the UVaSCF hardware boundary. This particular boundary represents the fuel-injector wall. While the CFD predicts a maximum $N_{H_{2} O}$ along this wall, the 
measured $N_{\mathrm{H}_{2} \mathrm{O}}$ is seen to plateau just before this boundary and then decrease significantly past $\mathrm{y}=-20 \mathrm{~mm}$. This is likely due to the fact that the CFD distributions shown are calculated at the actual exit of the UVaSCF hardware, while the TDLAT measurements were acquired slightly downstream of this point. This axial distance allows for room air to be entrained into the area defined by the UVaSCF hardware, which is extremely cold and dry in comparison to the core-flow of the scramjet. As such, it is indeed expected that the measured quantities of temperature, water vapor number density, and water vapor mole fraction would decrease toward ambient conditions past the defined hardware boundaries. The horizontal profile comparing the CFD and TDLAT water vapor number densities also agree extremely well, both in magnitude and shape. The profiles are within $6 \%$ of each other from $\mathrm{z}=-17 \mathrm{~mm}$ to $\mathrm{z}=15 \mathrm{~mm}$. It is seen that the CFD predicts the $N_{\mathrm{H}_{2} \mathrm{O}}$ to be relatively constant toward the walls, then increase to a maximum near the center of the duct. This same contour is observed in the TDLAT measurements. The water vapor number density gradients predicted by the CFD and measured by the TDLAT match extremely well. This demonstrates that the TDLAT technique is capable of measuring sharp gradients.

The third row of Figure 4.25 compares the simulated and measured water vapor mole fractions (calculated from the temperature and water vapor number density for TDLAT). Again, because $X_{\mathrm{H}_{2} \mathrm{O}}$ is derived from the measured temperature, larger discrepancies are observed between the CFD and TDLAT. It is encouraging how similar the profiles are in shape, correctly predicting the penetration in the vertical profile, and the symmetry seen in the horizontal profile.

The uncertainty analysis for TDLAT reconstructions of case 2 followed the procedure described in Section 3.4. 560 individual LOS measurements were collected at case 2 conditions. These measurements were collected along the same path as described for the stationary measurements in case 1. Figure 4.26 shows the resulting integrated absorbance values for the three absorption features over 560 LOS. 


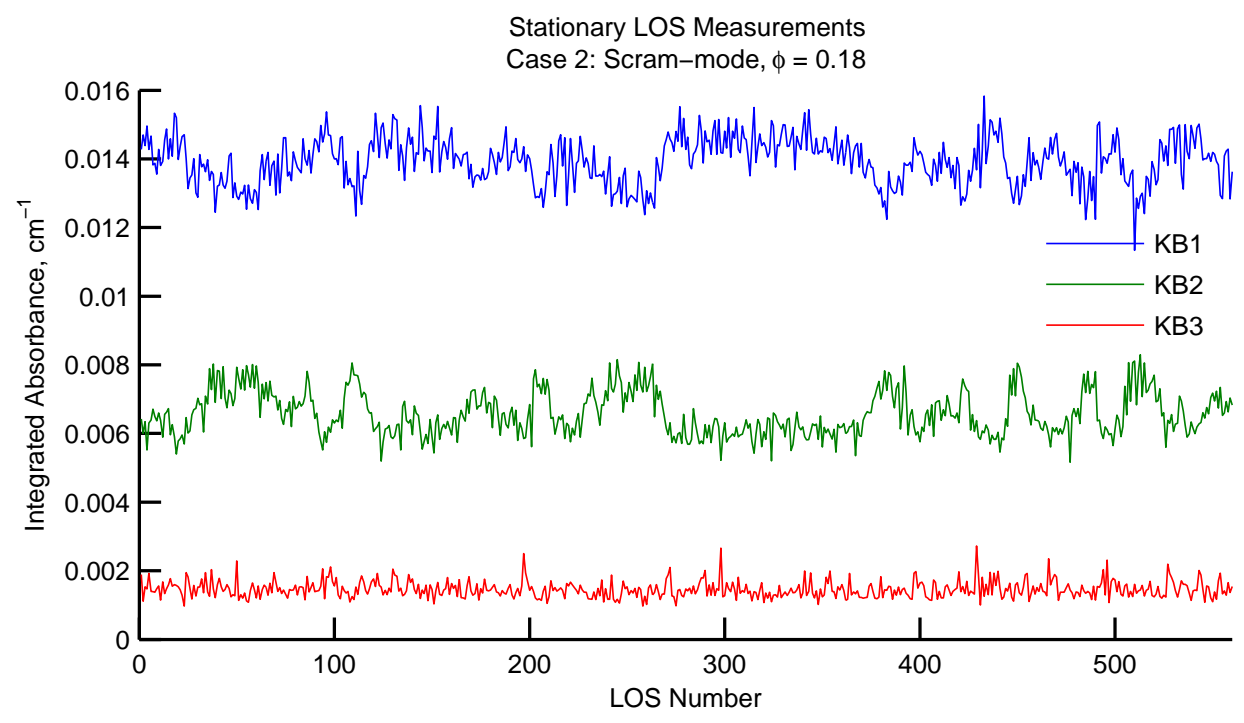

Figure 4.26: 560 stationary LOS collected at case 2 conditions.

From these stationary LOS measurements, the mean, standard deviation, and \% noise of the integrated absorbance for absorption features KB1, KB2, and KB3 were determined and are tabulated in Table 4.3.

TABLE 4.3: Statistical properties of case 2 measurements.

\begin{tabular}{llll}
\hline Absorption Feature & $\begin{array}{l}\text { Mean, } \mathrm{cm}^{-1} \\
\bar{A}\end{array}$ & $\begin{array}{l}\text { Standard Deviation, } \mathrm{cm}^{-1} \\
\sigma_{L O S}\end{array}$ & $\begin{array}{l}\text { \% Noise } \\
\sigma_{L O S} / \bar{A}\end{array}$ \\
\hline KB1 & .013927 & .00069059 & $4.96 \%$ \\
KB2 & .0065805 & .00063917 & $9.71 \%$ \\
KB3 & .0014581 & .00024814 & $17.02 \%$ \\
\hline
\end{tabular}

It is observed that the standard deviation and \% noise for these measurements are slightly higher than the non-combusting measurements acquired during the $12 \%$ steam injection conditions of case 1. Following the procedure outlined in Section 3.4, the uncertainties and $\%$ uncertainties (the uncertainty compared to the actual values) for the case 2 measurements shown in Figure 4.23 are shown in Figure 4.27. 


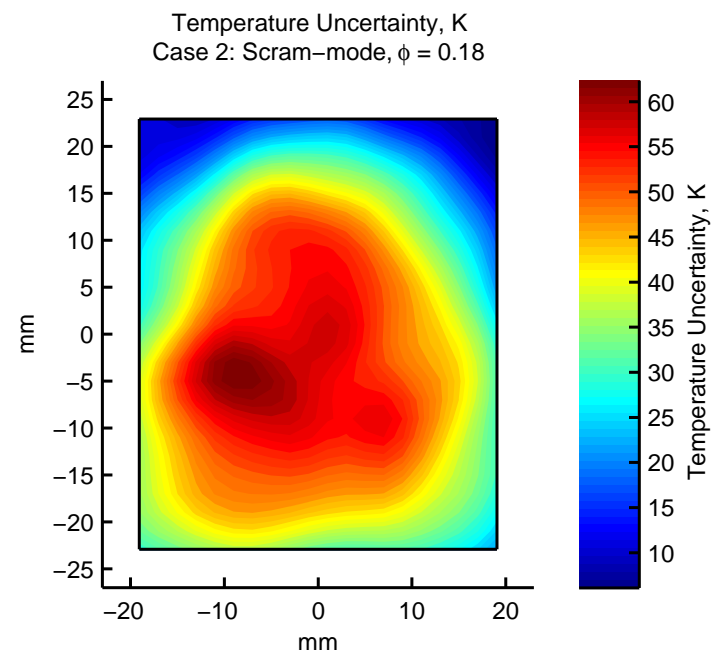

(a)

Reconstructed $\mathrm{H}_{2} \mathrm{O}$ Number Density Uncertainty, molecules $/ \mathrm{cm}^{3}$ Case 2: Scram-mode, $\phi=0.18$

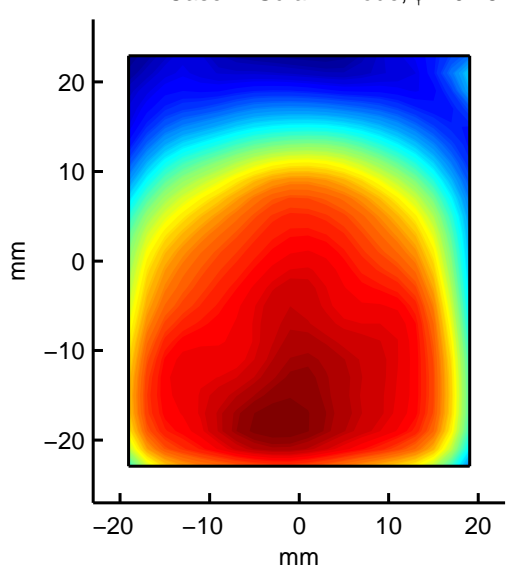

(c)

Reconstructed $\mathrm{H}_{2} \mathrm{O}$ Mole Fraction Uncertainty

Case 2: Scram-mode, $\phi=0.18$

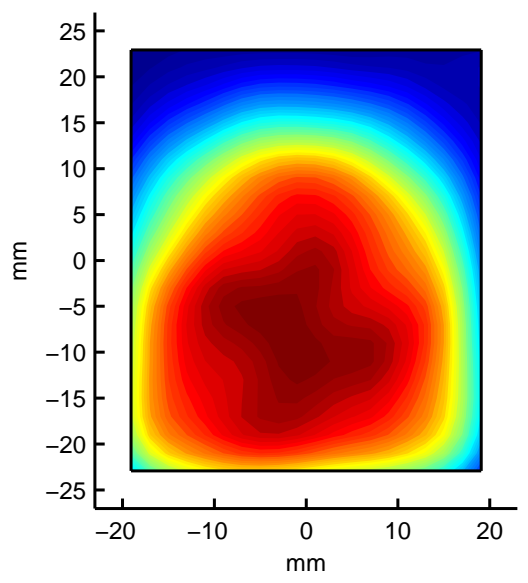

(e)

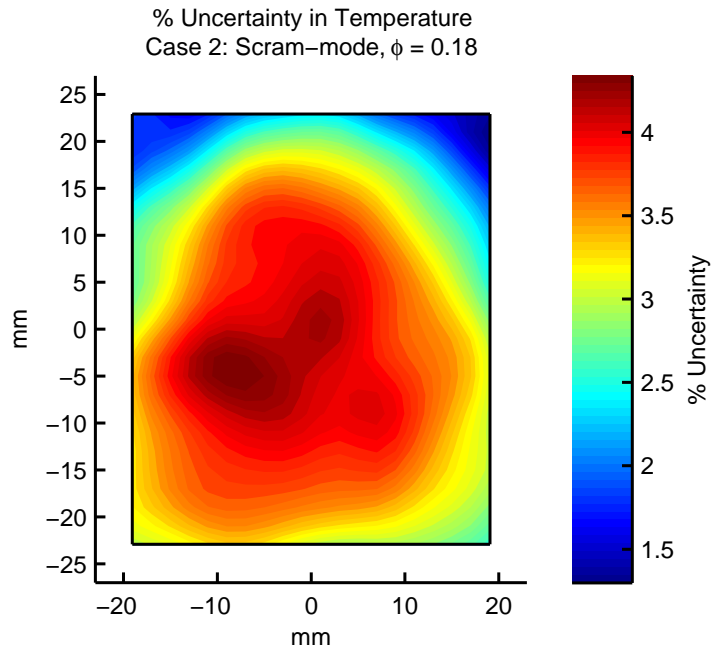

(b)

\% Uncertainty in $\mathrm{H}_{2} \mathrm{O}$ Number Density Case 2: Scram-mode, $\phi=0.18$
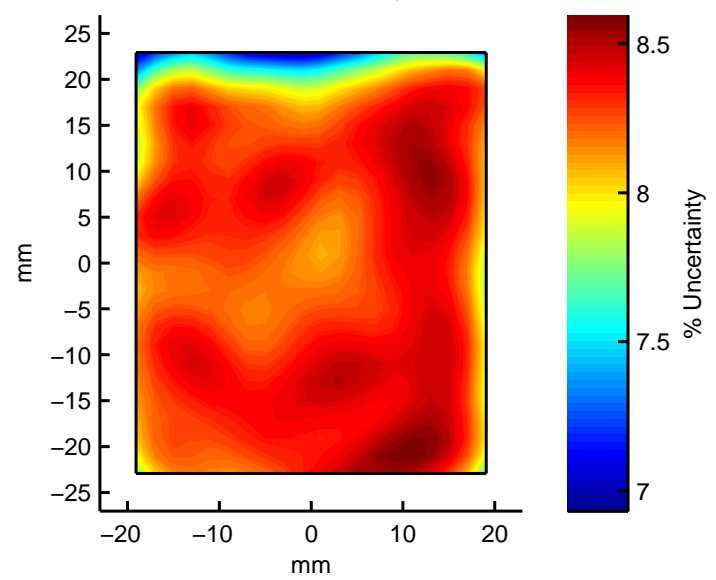

(d)

\% Uncertainty in $\mathrm{H}_{2} \mathrm{O}$ Mole Fraction

Case 2: Scram-mode, $\phi=0.18$

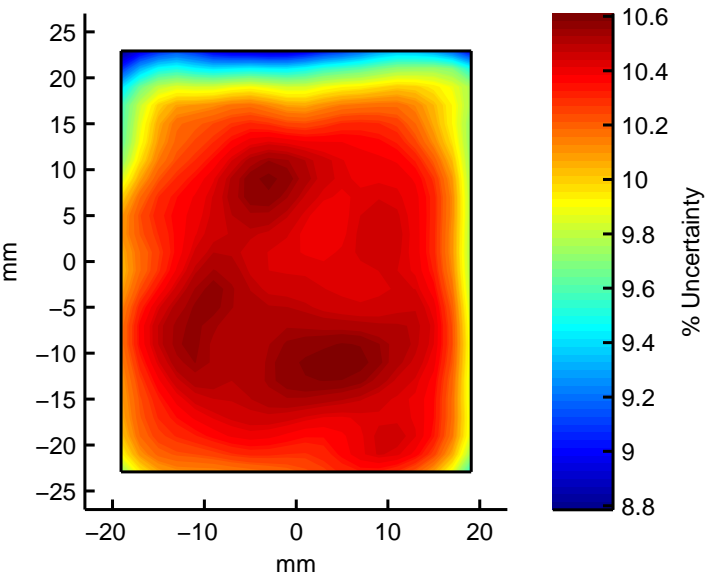

(f)

Figure 4.27: Absolute uncertainty and \% of full value uncertainty for case 2 reconstructions of temperature, water vapor number density, and water vapor mole fraction. 


\subsection{Case 3: Ram-mode $\phi=0.49$ Results}

TDLAT measurements were also acquired in ram-mode operation of the UVaSCF, with the test conditions listed in Table 4.1. In the ram-mode, a series of oblique shockwaves, called a shock train, are located in the isolator section, upstream of the fuel-injector. This series of shockwaves serve to decelerate part of the flow causing mixed subsonic/supersonic flow to enter the combustor section.

The measured sinograms for case 3, acquired with the TDLAT system and before background subtraction, are shown in Figure 4.28. Again, the left column consists of raw measured data, while the right column shows the minimally-filtered sinograms. The KB1 sinogram actually shows slightly lower values of absorbance, as compared to the case 2 data, suggesting that the temperatures observed in this ram-mode case are significantly above the temperature at which KB1's line strength is maximized. This was illustrated in Figure 2.5a, which shows each of the transitions' temperature dependence of line strength. Figure 4.29 shows the calculated temperatures for each LOS measurement obtained for case 3. Indeed, it is seen that several of the individual LOS measurements are above 1,500 K, which is higher than the temperature at which KB1's line strength is maximized.

The background absorbance was then subtracted, the ML-EM reconstruction routine was executed, and the distributions of the four absorption features were obtained, as shown in Figure 4.30 . 


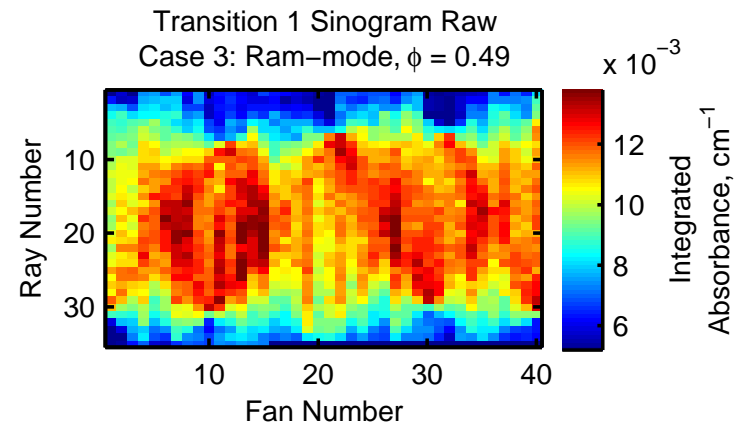

(a)

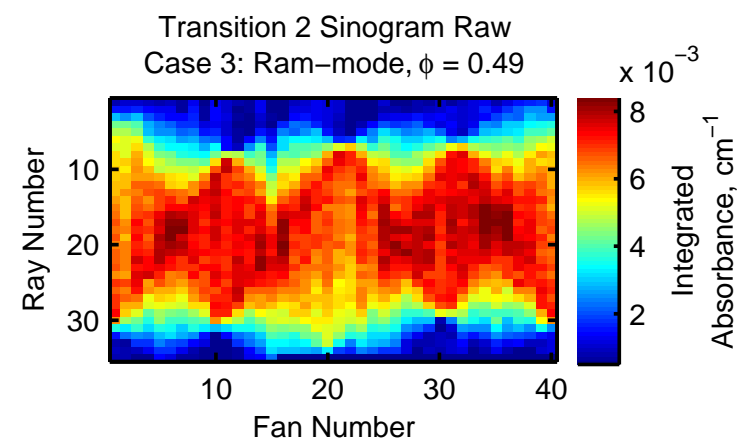

(c)

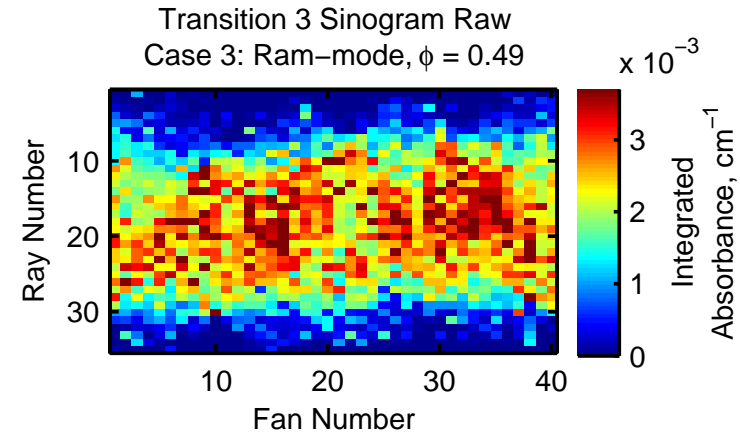

(e)

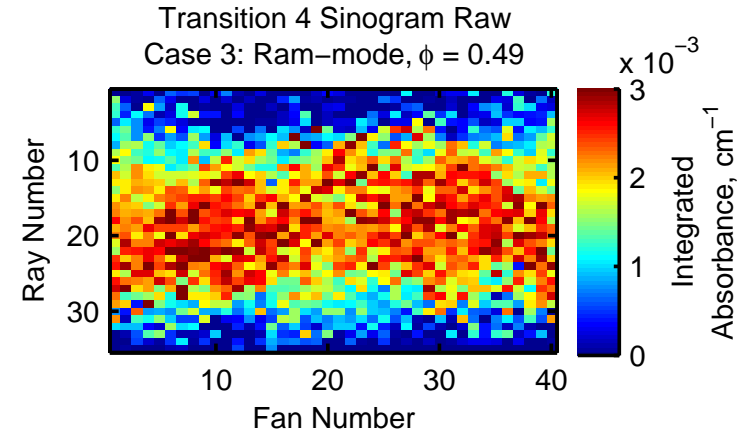

(g)

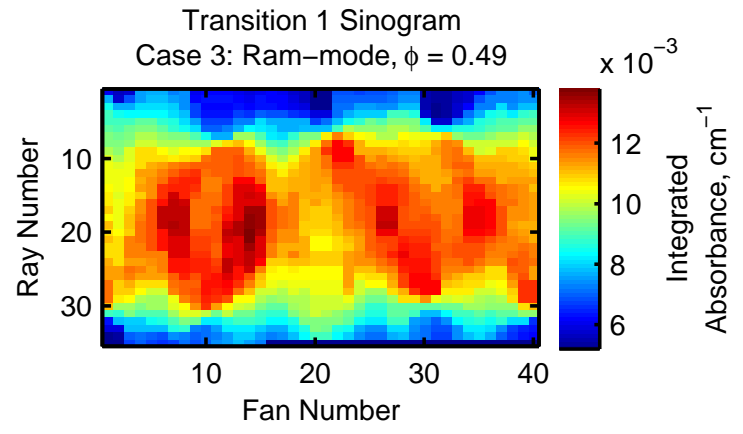

(b)

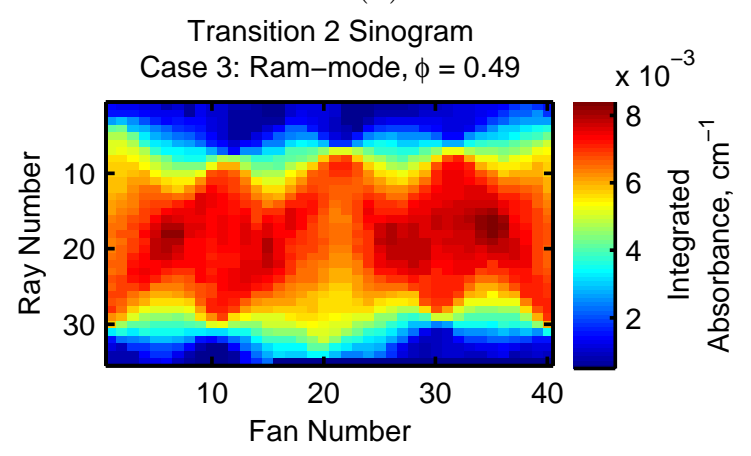

(d)

Transition 3 Sinogram

Case 3: Ram-mode, $\phi=0.49$

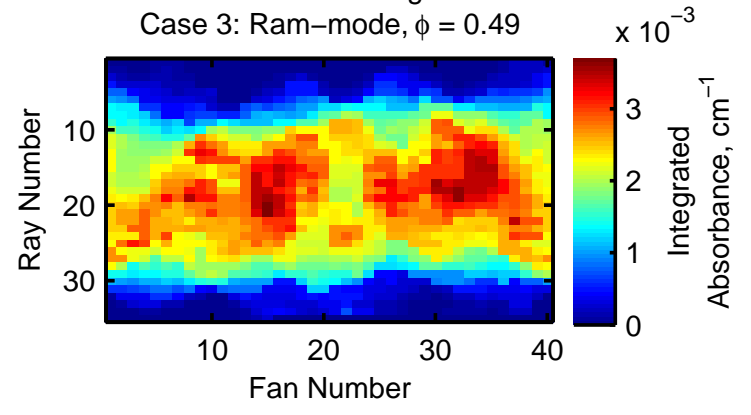

(f)

Transition 4 Sinogram

Case 3: Ram-mode, $\phi=0.49$

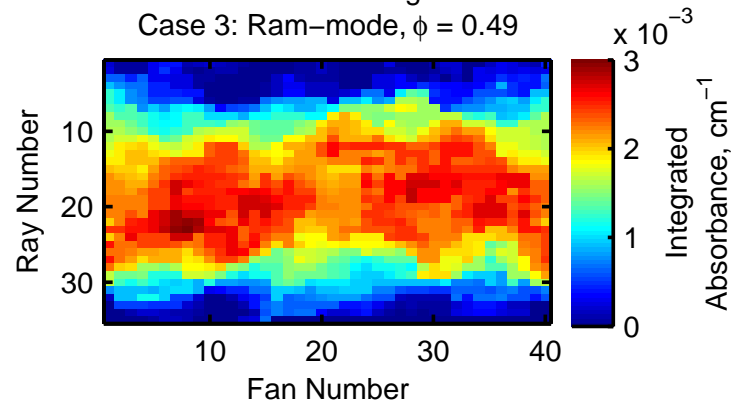

(h)

FiguRE 4.28: Integrated absorbance sinograms at the exit plane of the UVaSCF for case 3. The first column contains the raw sinograms of TDLAT measured integrated absorbance for each of the four absorption features (KB1-KB4) and the second column contains the corresponding filtered sinograms. 


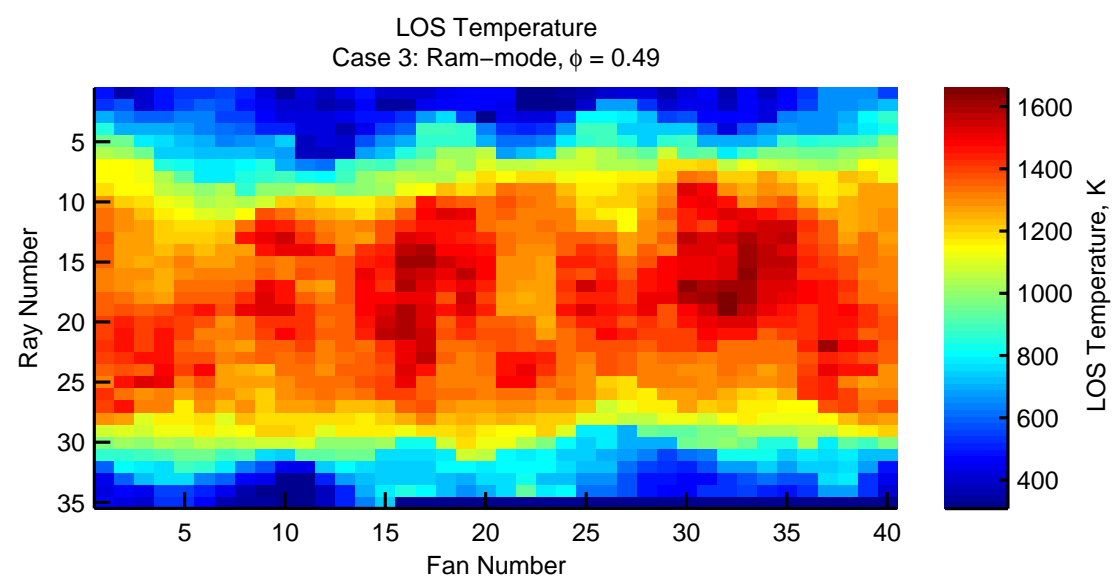

FIGURE 4.29: LOS temperatures calculated for case 3 TDLAT measurements.

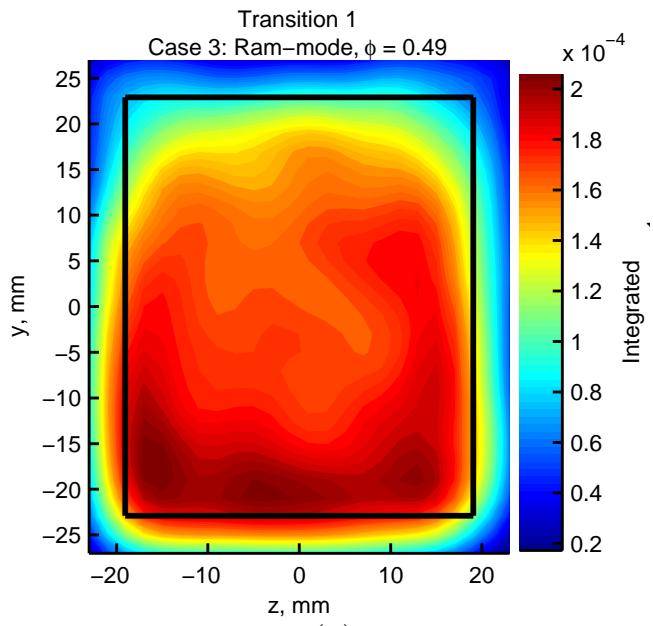

(a)

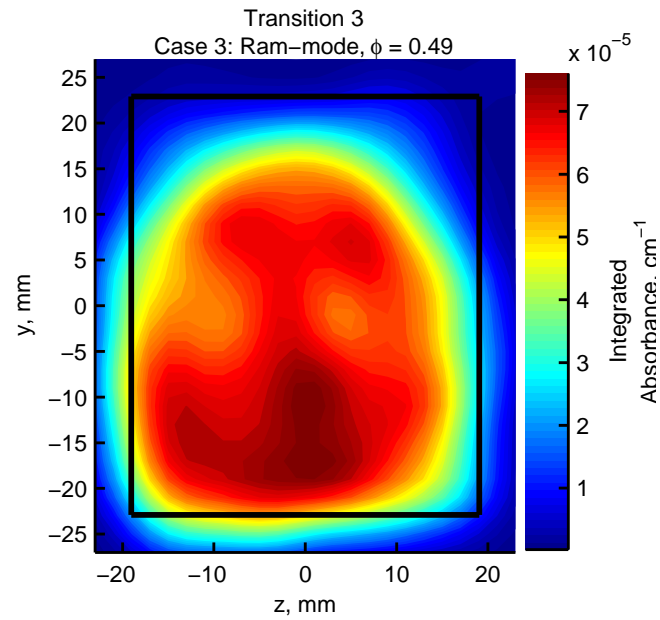

(c)

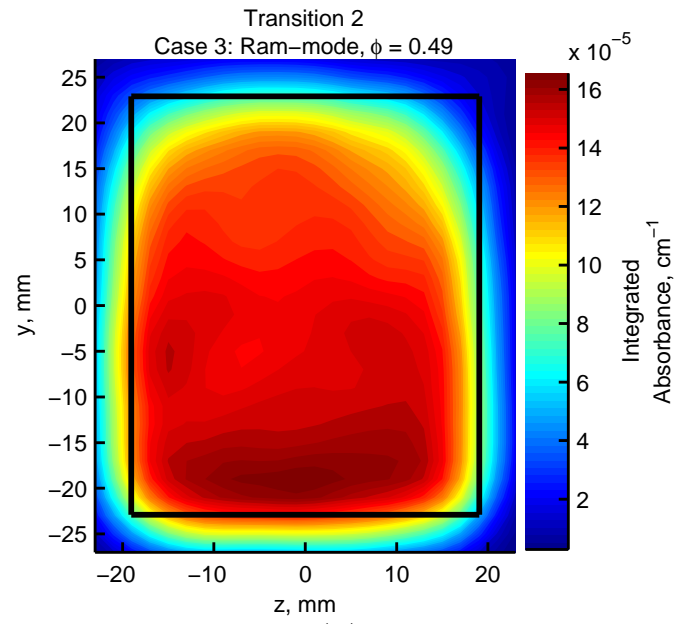

(b)

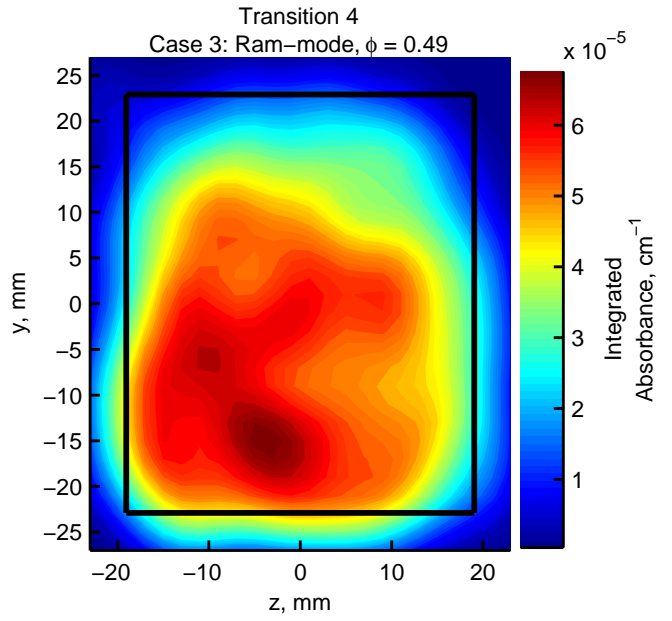

(d)

FigURE 4.30: Reconstructed integrated absorbance at the exit plane of the UVaSCF for case 3, for (a) KB1, (b) KB2, (c) KB3, and (d) KB4. 
Two potential relatively cool spots are seen in Figures $4.30 \mathrm{~b}$ and $4.30 \mathrm{c}$ near $(-10,-3)$ $\mathrm{mm}$ and $(10,3) \mathrm{mm}$. It is expected that these two locations may be lower in temperature than their surroundings because the KB2 transition is locally high and the KB3 transition is locally low. Compared to the integrated absorbance distributions of case 2 , these distributions of integrated absorbance are seen to extend much further across the duct. While Figure 4.30a shows over all lower magnitudes of KB1 integrated absorbance (as was discussed earlier), the reconstructed integrated absorbances of the other three absorption features are all higher in magnitude, suggesting that case 3 will likely result in larger water vapor number densities.

As with the other measurement sets, the two-line thermometry temperatures can be obtained via Equation 2.10 and various combinations of the three absorption features, as is shown in Figure 4.31.

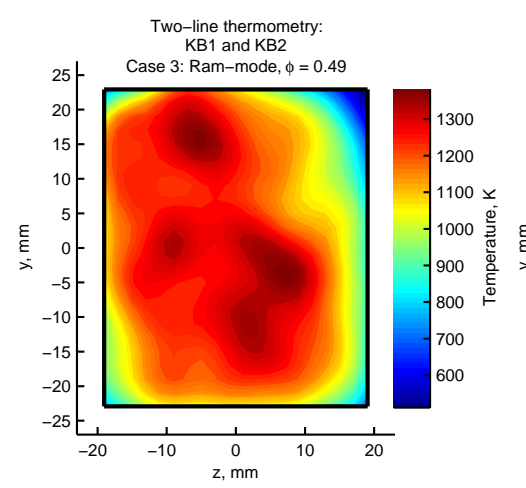

(a)

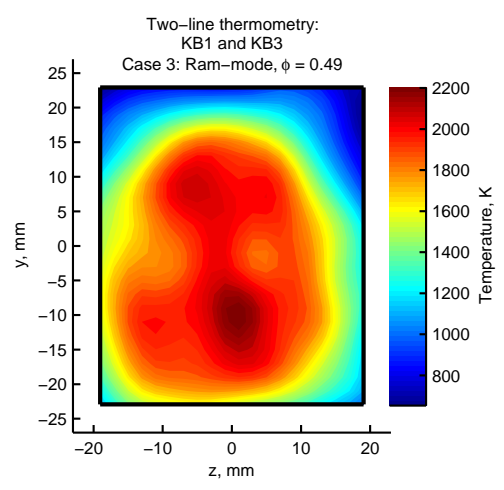

(b)

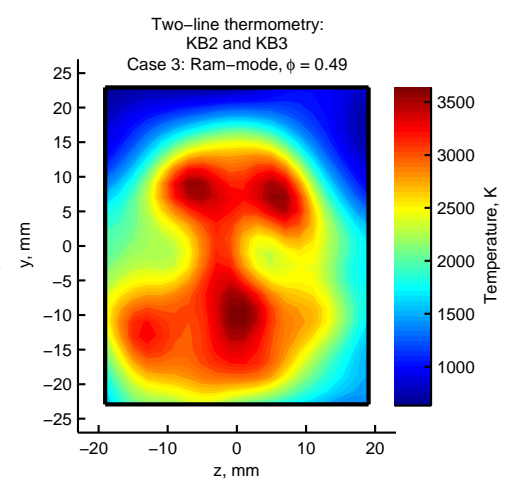

(c)

Figure 4.31: Two-line thermometry calculation via Equation 2.10 at the exit plane of the UVaSCF for case 3, for line-pairs (a) KB1 and KB2, (b) KB1 and KB3, and (c) KB2 and KB3.

The Boltzmann plot method was utilized for the KB1, KB2, and KB3 absorption features. The RMSE of the linear fit was recorded for all pixels in the distribution and is seen in Figure 4.32. Again, within the limits of the black rectangle (representing the UVaSCF flowpath) low overall error is seen, suggesting that the measured absorbance features are very collinear (in log-space) for these pixels. 


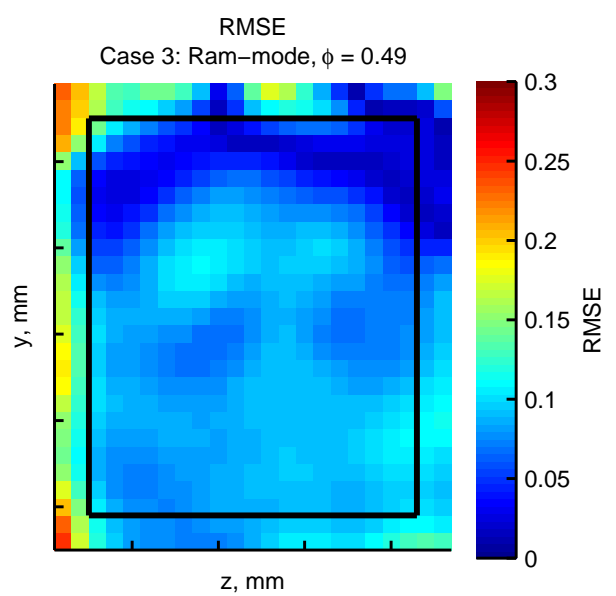

FigURE 4.32: Root-mean square error for Boltzmann plot at the exit plane of the UVaSCF for case 3 .

The resulting temperature, water vapor number density, and water vapor mole fraction distributions for case 3 are shown in Figure 4.33. The temperatures observed are significantly higher than those seen in case 2, as is expected. The maximum temperature in the duct is $2170 \mathrm{~K}$ and the mean temperature across the duct is $1580 \mathrm{~K}$. The maximum and mean temperatures observed for this ram-mode case are significantly higher than those observed for the $12 \%$ steam injection case and the scram-mode case, which showed maximums of $965 \mathrm{~K}$ and $1445 \mathrm{~K}$ and means of $880 \mathrm{~K}$ and $1145 \mathrm{~K}$, respectively. As was predicted earlier, the high-temperature region extends across most of the duct in the y-direction. Two locally cold regions are seen near the predicted locations of $(-10,-3) \mathrm{mm}$ and $(10,3) \mathrm{mm}$, showing temperatures approximately 150-200 K lower than the surrounding flow. These depressions are even more evident in Figures 4.33b and 4.33c, where the local minima are nearly $20 \%$ lower than their neighboring pixels. 


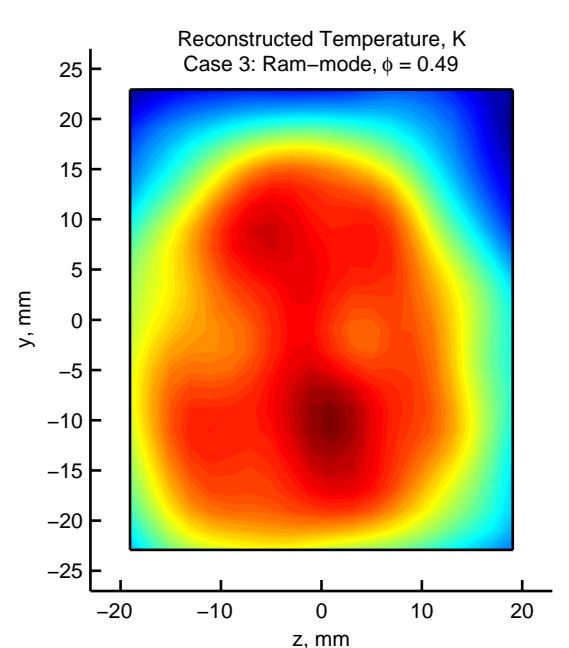

(a)
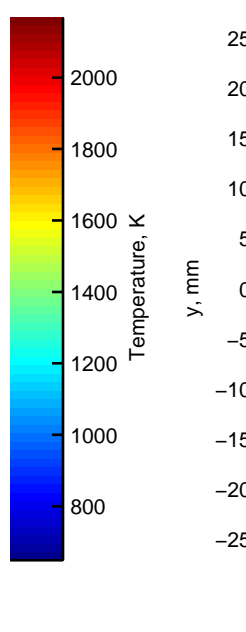

$$
\text { - }
$$

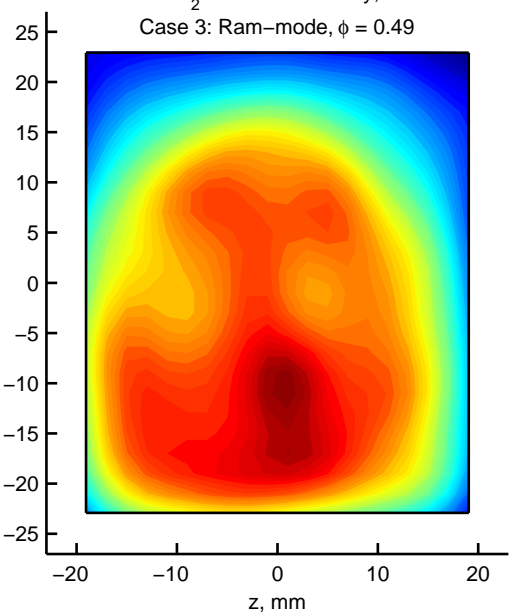

(b)

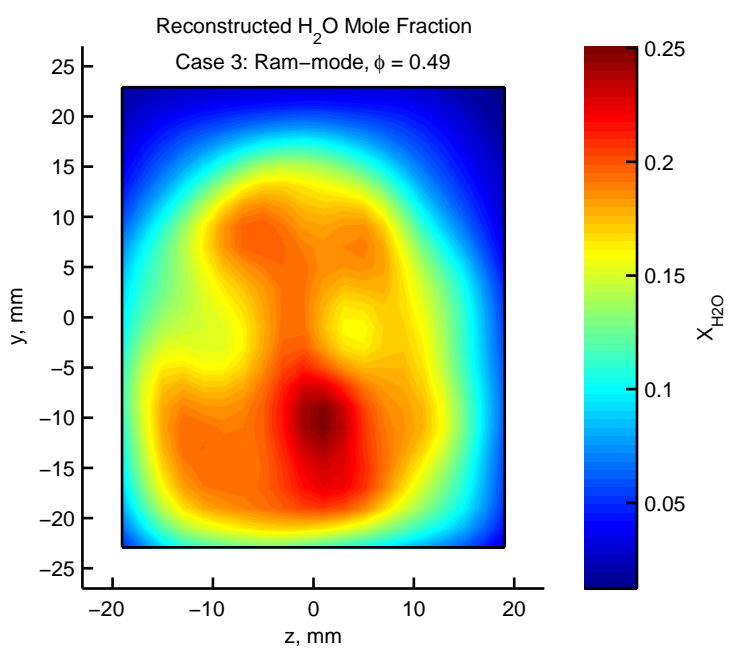

(c)

Figure 4.33: Final reconstructions at the exit plane of the UVaSCF for case 3: (a) temperature, (b) water vapor number density, and (c) water vapor mole fraction. 
To further explore these local minima, SPIV measurements collected at the same exit plane and test conditions for the ram-mode case (case 3) by Rice are examined. Figure 4.34a shows the z-component of velocity, $V_{z}$, at the exit plane of the UVaSCF, while Figure 4.34b shows a contour plot of water vapor mole fraction measured by TDLAT (and previously shown in Figure $4.33 \mathrm{~b})$ with the in-plane velocity vectors $\left(V_{z}\right.$ and $V_{y}$ ) overlaid, courtesy of Rice [60]. Maximum axial velocities $\left(V_{x}\right)$ of $\sim 785 \mathrm{~m} / \mathrm{s}$ are observed, with a mean near $375 \mathrm{~m} / \mathrm{s}$. Therefore, the magnitude of these cross-plane velocities are generally $<10 \%-15 \%$ of the axial flow. There is a significant amount of air entrained from the surrounding atmosphere, shown as dark blue and dark red in the $V_{z}$ distribution. Also noticeable is the hourglass shape of this entrained air. It is observed that the entrained air seems to "pinch" in the flowfield, most strongly near $\mathrm{y}=0 \mathrm{~mm}$, and decreasing in $\pm \mathrm{y}$. It is also seen that the magnitude of $V_{z}$ is larger on the left-side of the duct than on the right.

These observations of the z-component velocity distribution are directly in line with the local depressions of temperature and water vapor number density measured by TDLAT. Figure 4.34b shows that these strong cross-plane velocities pinch in the flow in the same location that the low number densities are observed. A stronger influence is seen on the left-side of the duct, both in $V_{z}$ and $N_{H_{2} O}$. It is postulated that the entrainment of room air reduces both the temperature and water vapor number density in these locations, as the room air is very cold and dry compared to the core flow of the facility. 


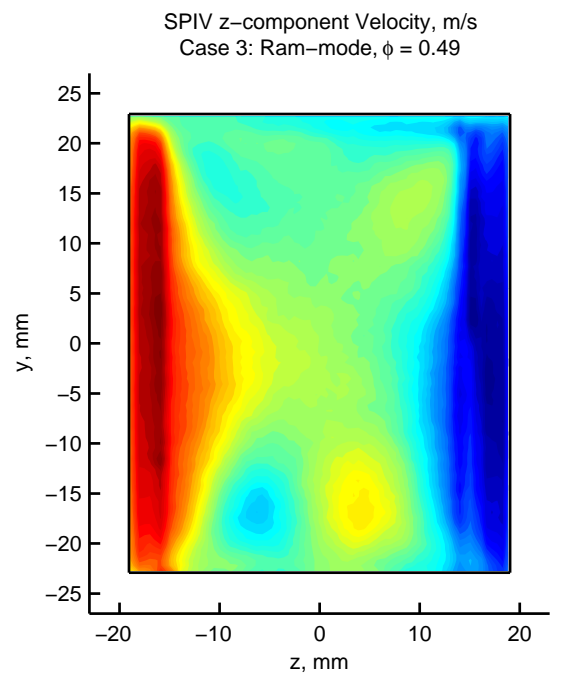

(a)

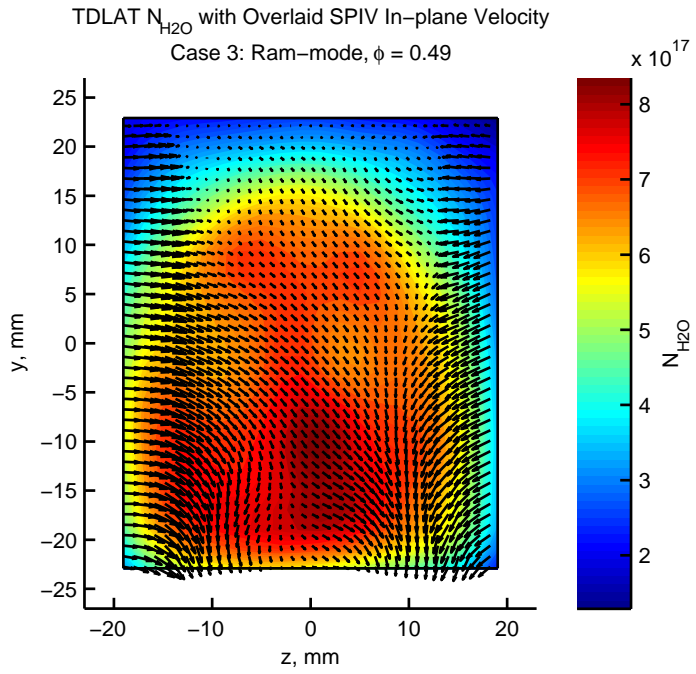

(b)

FIgURE 4.34: (a) Z-component of velocity, $V_{z}$, measured by Rice [60] at the exit plane of the UVaSCF for case 3, and (b) contour plot of water vapor mole fraction via TDLAT with overlaid in-plane velocity vectors via SPIV [60] at the exit plane of the UVaSCF for case 3.

Line plots of the temperature, water vapor number density, and water vapor mole fraction for case 3 acquired by TDLAT are seen in Figure 4.35. These line plots serve to further elucidate the structure of the measured quantities. No CFD distributions were available for case 3, and thus only the TDLAT measurements are seen in Figure 4.35. The temperature profiles show maximum temperatures near 2,200 K, with penetration across nearly $90 \%$ across the duct. The horizontal profiles of temperature, $\mathrm{H}_{2} \mathrm{O}$ number density, and $\mathrm{H}_{2} \mathrm{O}$ mole fraction show contours similar to those which were observed for case 2: relatively flat values near the edges of the duct, with a peak found in the center. This peak value is more exaggerated in the ram-mode distributions of case 3 than it was in the scram-mode distributions of case 2 .

The water vapor mole fraction profiles are seen to have maxima nearing $25 \%$ which are significantly higher than the maximum of $10.4 \%$ seen in the scram-mode case. This is consistent with the observation of higher overall values of integrated absorbance seen both in the measured sinograms and reconstructed absorbance distributions. It also agrees with the higher amount of $\mathrm{H}_{2}$ fuel being injected into the UVaSCF for this case. 


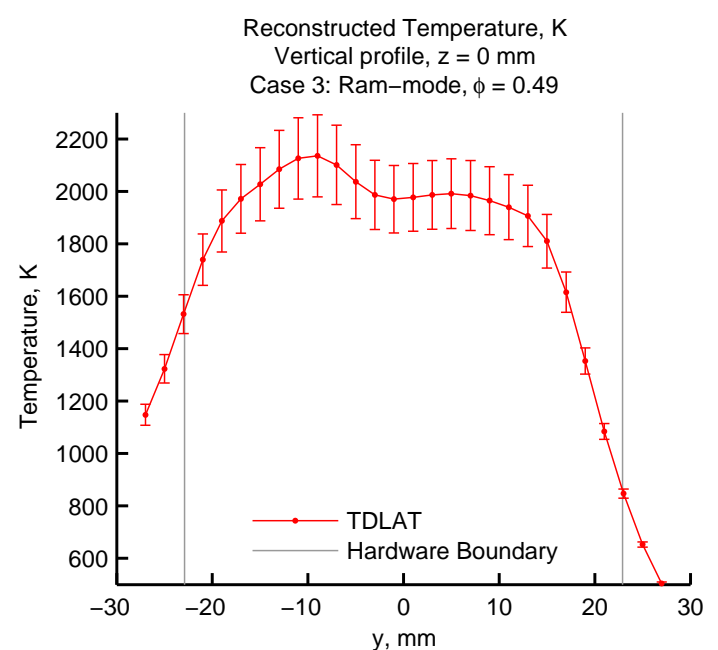

(a)

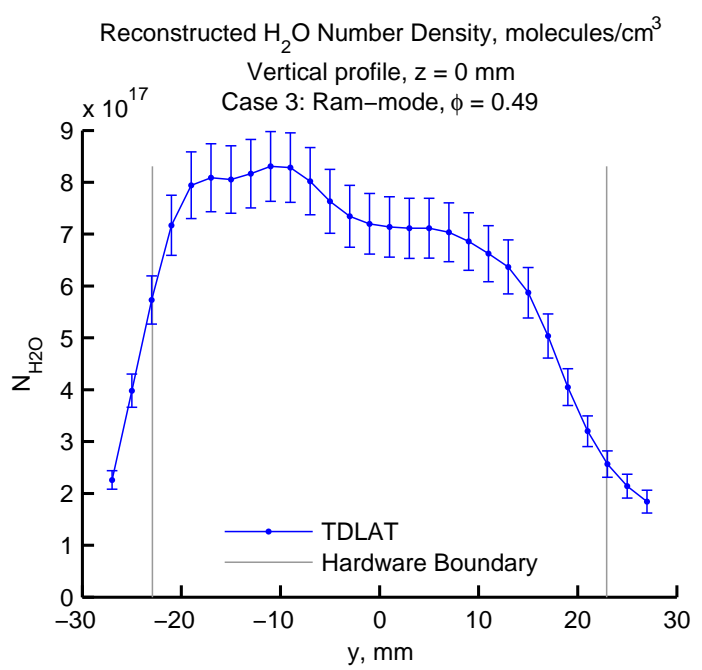

(c)

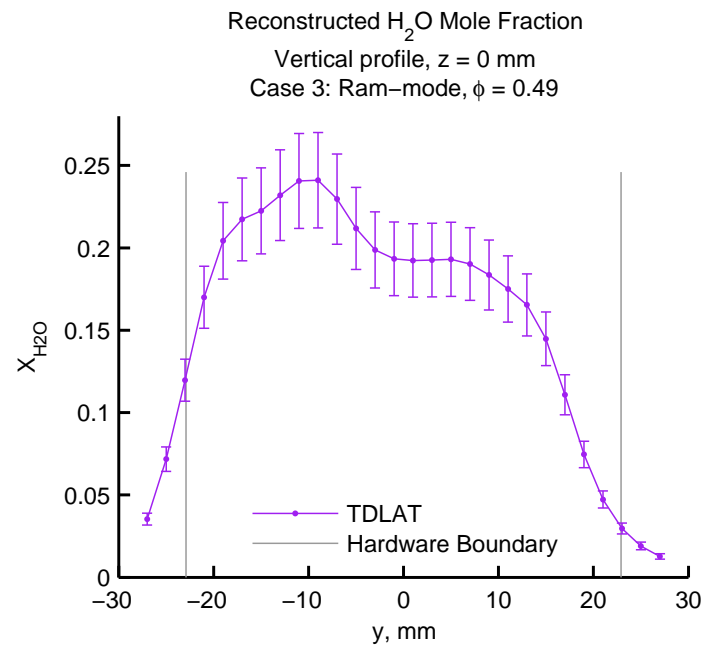

(e)

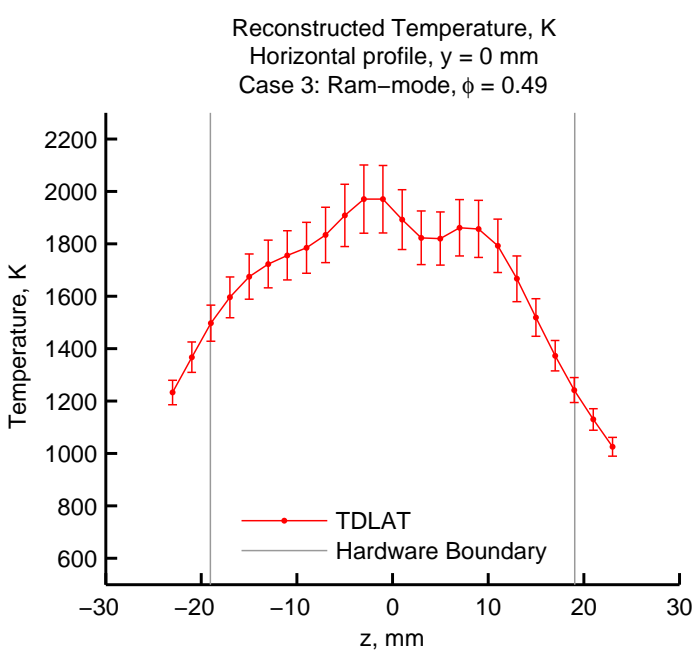

(b)

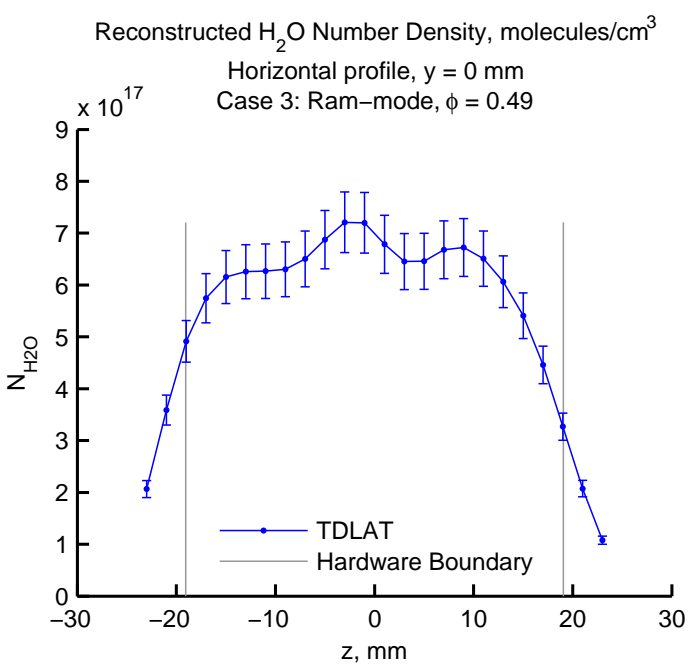

(d)

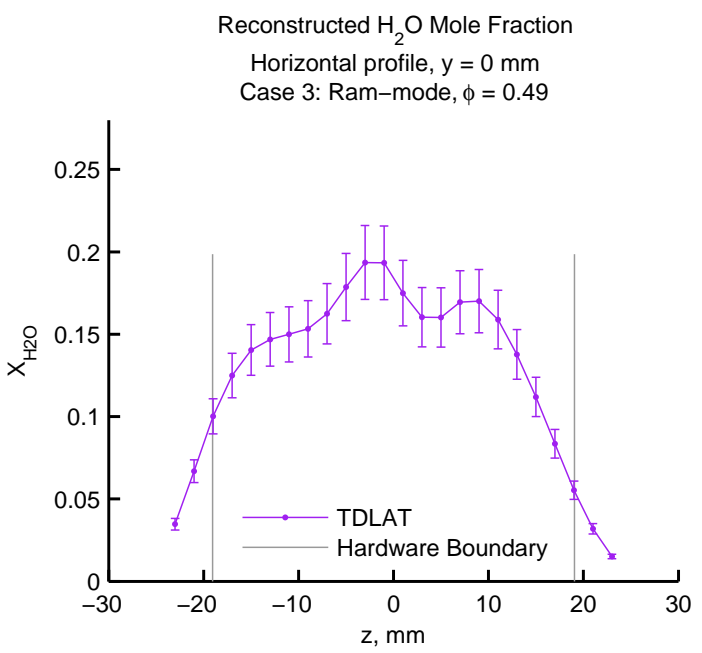

(f)

Figure 4.35: Case 3 line plots (profiles) both vertical and horizontal of (a) vertical temperature profile, (b) horizontal temperature profile, (c) vertical water vapor number density profile, (d) horizontal water vapor number density profile, (e) vertical water vapor mole fraction profile, and (f) horizontal water vapor mole fraction profile. 
For case 3 data, the uncertainty was quantified using the measured stationary LOS from case 2 because stationary measurements were not able to be acquired for the case 3 test conditions due to limited fuel availability during testing. The standard deviation of the integrated absorbance was shown in Figure 4.26 and summarized in Table 4.3. The error analysis procedure detailed in Chapter 3 was utilized with the measured distributions of case 3 and the resulting uncertainties for temperature, water vapor number density, and water number mole fraction are shown in Figure 4.36 . 


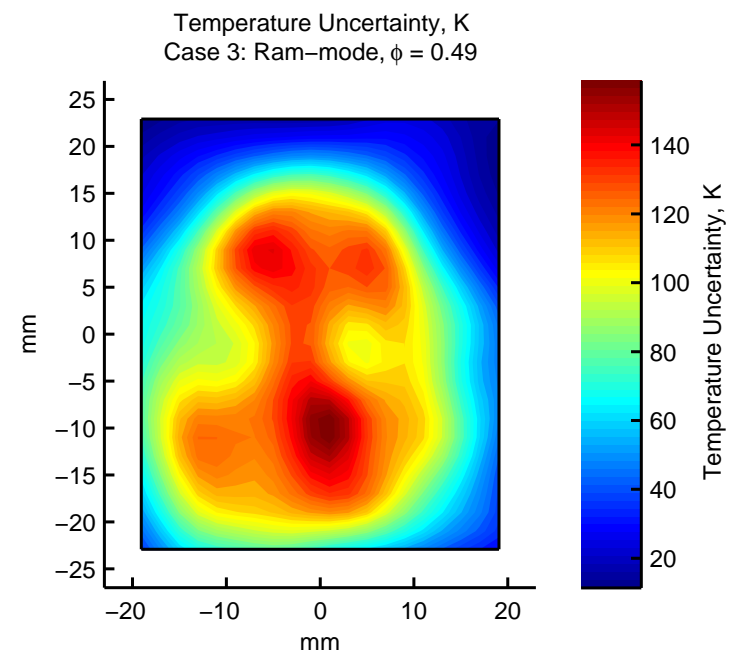

(a)

Reconstructed $\mathrm{H}_{2} \mathrm{O}$ Number Density Uncertainty, molecules $/ \mathrm{cm}^{3}$

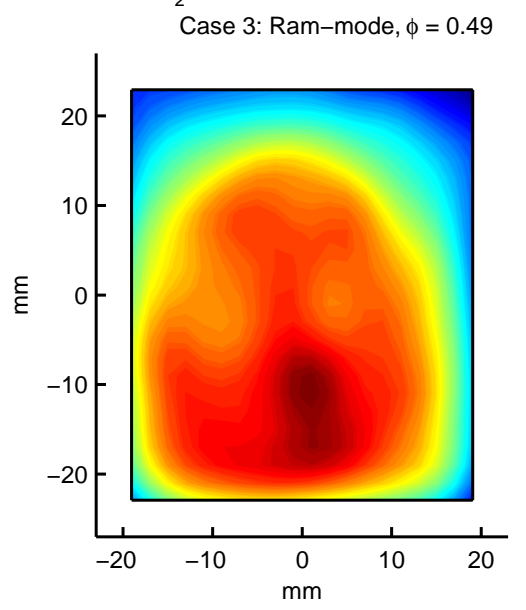

(c)

Reconstructed $\mathrm{H}_{2} \mathrm{O}$ Mole Fraction Uncertainty Case 3: Ram-mode, $\phi=0.49$

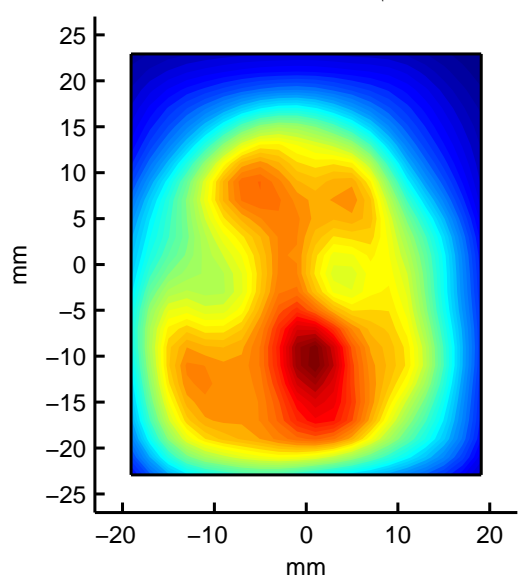

(e)

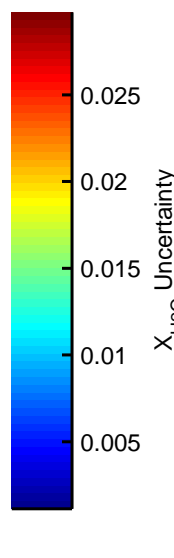

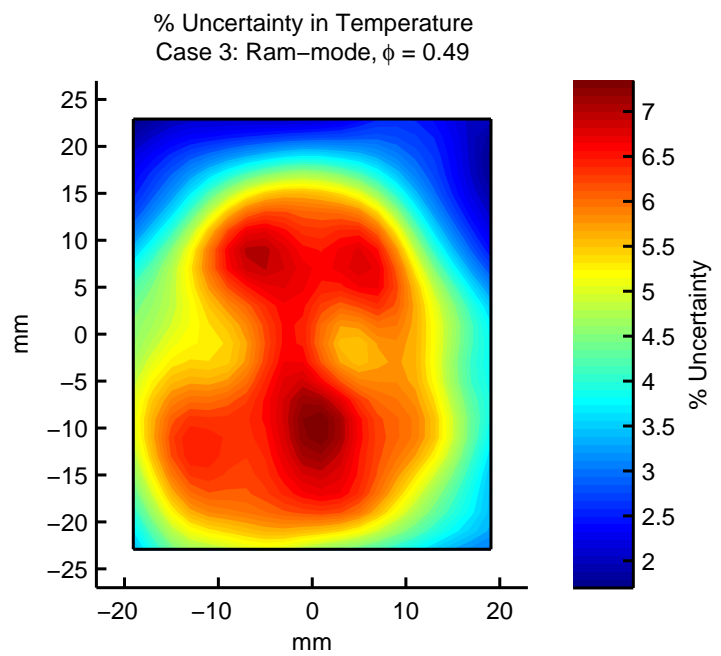

(b)

\% Uncertainty in $\mathrm{H}_{2} \mathrm{O}$ Number Density Case 3: Ram-mode, $\phi=0.49$

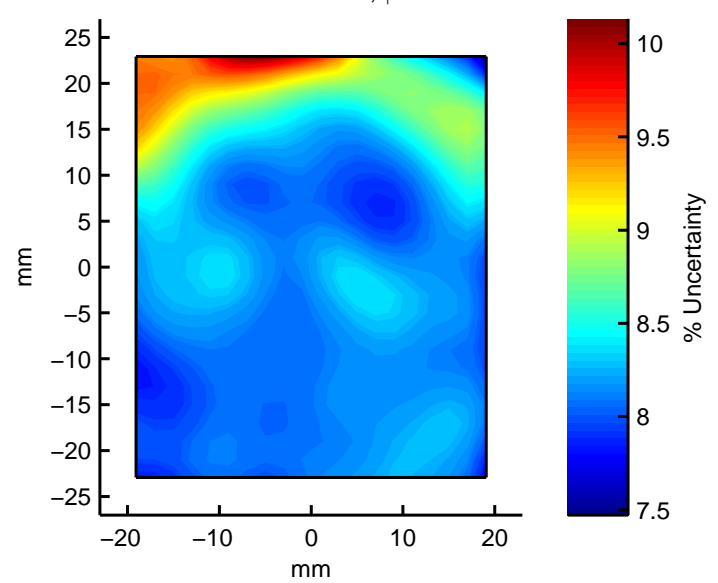

(d)

\% Uncertainty in $\mathrm{H}_{2} \mathrm{O}$ Mole Fraction

Case 3: Ram-mode, $\phi=0.49$

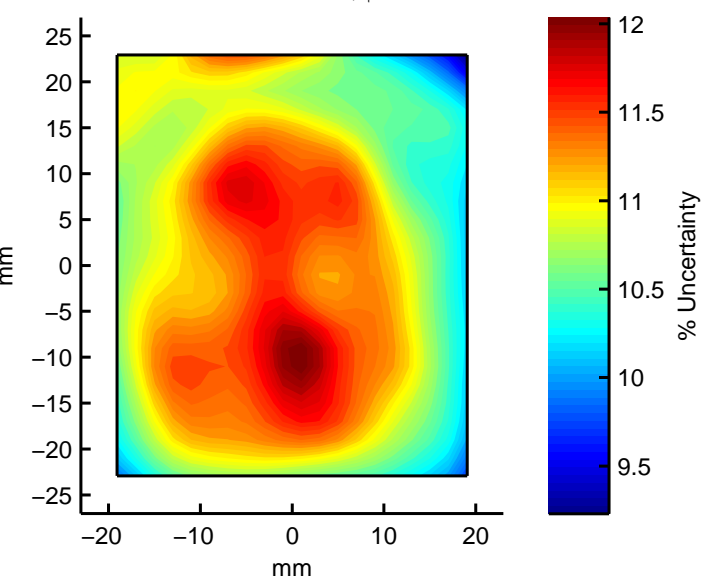

(f)

Figure 4.36: Absolute uncertainty and \% of full value uncertainty for case 3 reconstructions of temperature, water vapor number density, and water vapor mole fraction. 


\subsection{Combustion Efficiency}

As described in Chapter 1, the combustion efficiency of a (sc)ramjet engine with $H_{2}$ combustion can be measured directly by measuring the spatially resolved water vapor number density and the spatially-resolved axial velocity, and utilizing Equation 1.1.

$$
\eta_{c}=\frac{\int_{A_{\text {exit }}} N_{H_{2} O}(y, z) V_{x}(y, z) \mathrm{d} A_{\text {exit }}}{\dot{N}_{\text {Hydrogen,injected }}}
$$

The spatially resolved axial velocity distribution for both the scramjet-mode (case 2) and ram-mode (case 3) has been measured by Rice via stereoscopic particle image velocimetry (SPIV). [60][61]

To calculate combustion efficiency, the water vapor number density distribution measured via TDLAT is converted from units of molecules $/ \mathrm{cm}^{3}$ to grams $/ \mathrm{m}^{3}$ by the conversion seen in Equation 4.1:

$$
N_{\mathrm{H}_{2} \mathrm{O}}\left[\frac{\text { grams }}{\mathrm{m}^{3}}\right]=N_{\mathrm{H}_{2} \mathrm{O}}\left[\frac{\text { molecules }}{\mathrm{cm}}\right] \cdot\left(\frac{100 \mathrm{~cm}}{1 \mathrm{~m}}\right)^{3} \cdot M W_{\mathrm{H}_{2}} \cdot \frac{1}{N_{A}}
$$

where $M W_{H_{2}}$ is twice the molecular weight of a hydrogen atom, $2 \times 1.00794$ grams $/$ mole. Next, according to Equation 1.1, the water vapor number density, the axial velocity, and the grid pixel area are multiplied at each grid point. This results in a distribution of water vapor flux through each pixel area. This distribution is then summed across the dimensions of the UVaSCF exit plane flowpath to calculate the total mass flow rate of $\mathrm{H}_{2} \mathrm{O}$ exiting the UVaSCF facility at the exit plane. The ratio of this experimentally measured mass flow rate to the UVaSCF facility's measured mass flow rate of injected $\mathrm{H}_{2}$ is then calculated, resulting in the percentage of combustion completeness at the UVaSCF exit plane. Uncertainties calculated for the experimental results are determined via Equations 3.5a-3.5m, with $\sigma V_{x}=8.8 \mathrm{~m} / \mathrm{s}$ according to Rice [60].

Following the previously outlined procedure, the combustion efficiency of the UVaSCF is calculated for both case 2 (the scram-mode) and case 3 (the ram-mode). As a point of comparison, the combustion efficiency of the UVaSCF is calculated for 


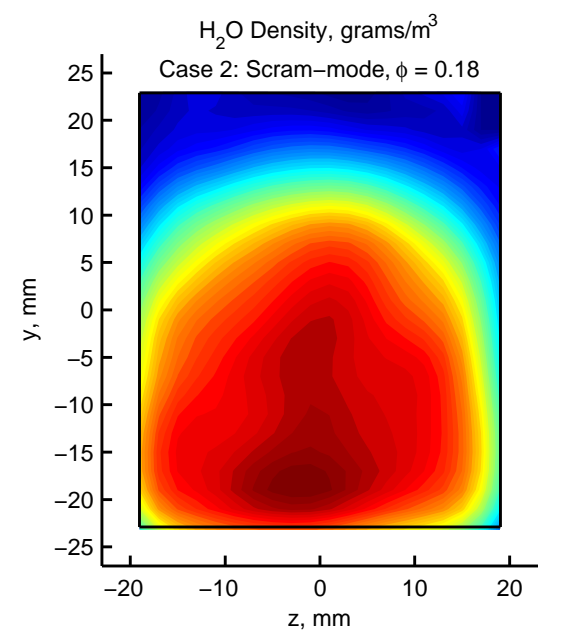

(a)

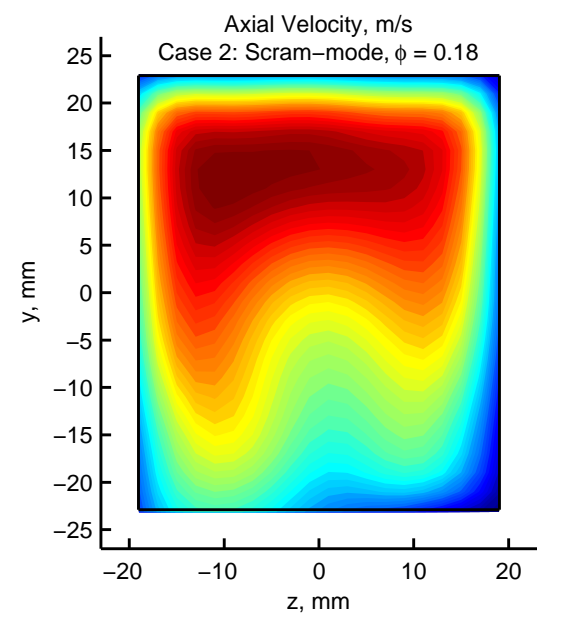

(c)

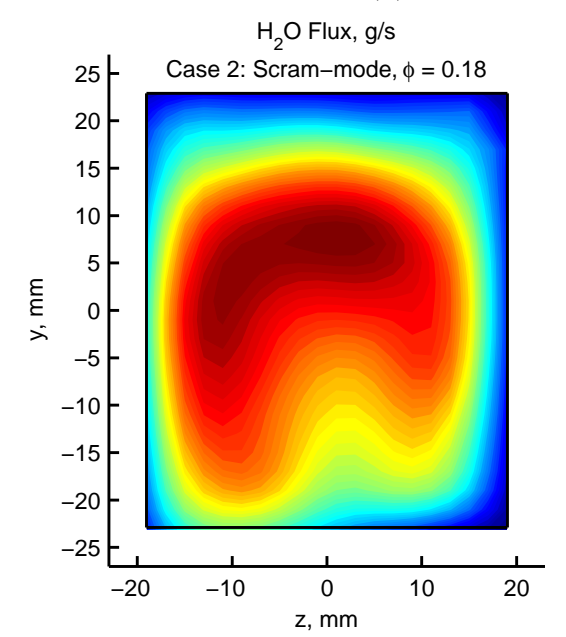

(e)

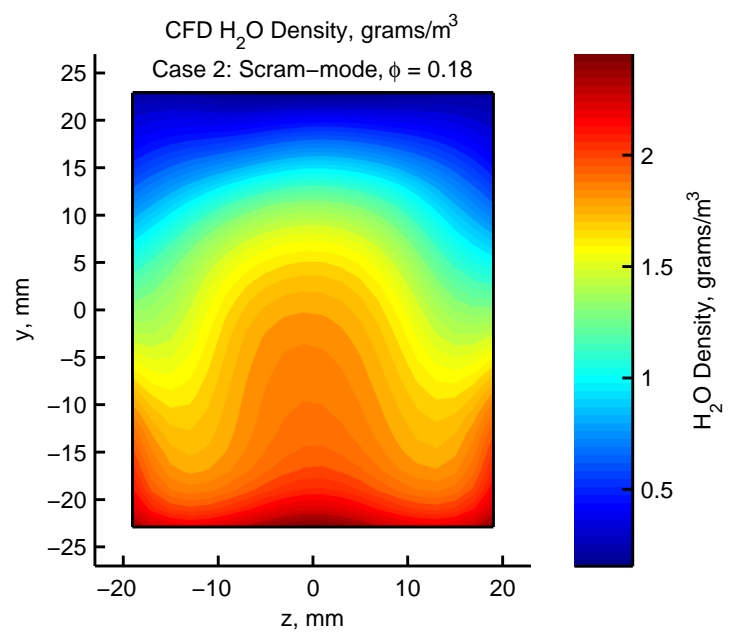

(b)
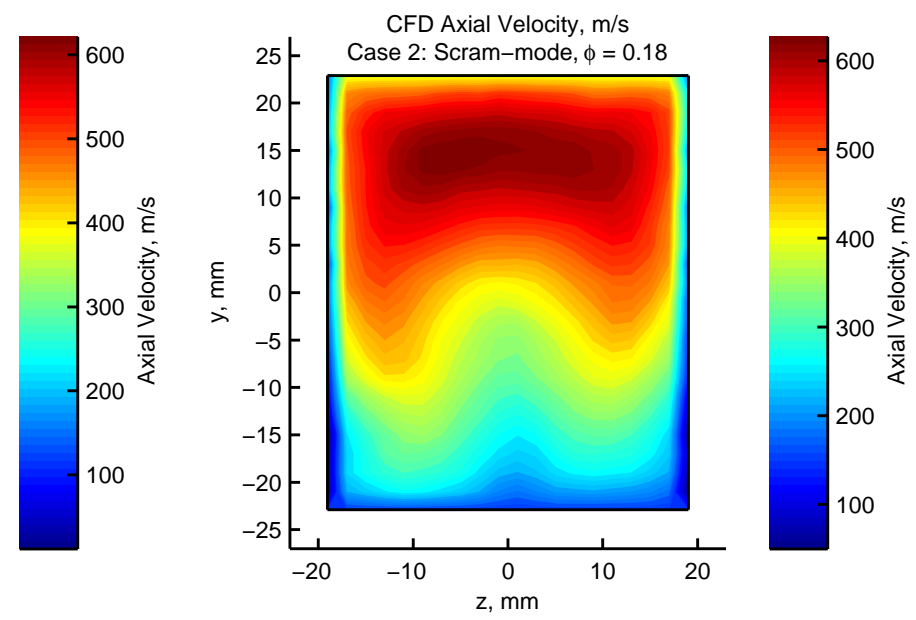

(d)
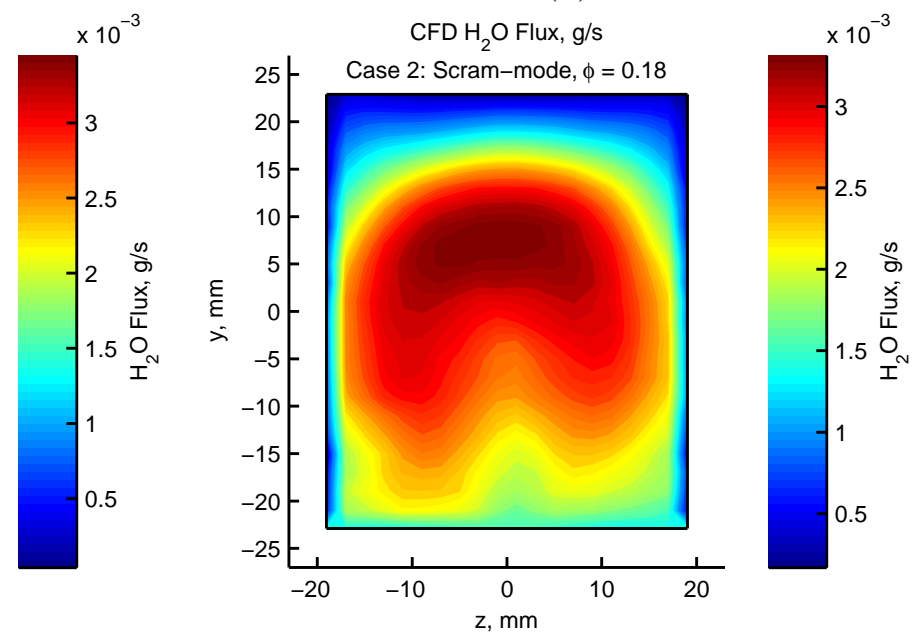

(f)

FiguRe 4.37: Water vapor number density, axial velocity, and water vapor flux for scram-mode operation from TDLAT (first column) and CFD [55] (second column). 
case 2 from the CFD distributions of axial velocity, static temperature, and water vapor mole fraction, interpolated onto the same $2 \mathrm{~mm} \times 2 \mathrm{~mm}$ grid as the experimental measurements.

Figure 4.37 shows the components of the combustion efficiency calculation for case 2. The left column shows the distributions directly measured by TDLAT and SPIV, while the right column shows the distributions computed by CFD. The first row shows the water vapor number density distributions, the second row shows the axial velocity distributions, and the third row shows the water vapor flux through each individual pixel. It is remarkable how similar the CFD distributions are to the experimentally measured distributions. Overall, the experimentally measured distributions are slightly more asymmetric than the CFD predicts. The comparison between the CFD calculated water vapor flux and the experimentally measured water vapor flux is exceptional, considering the level of complexity involved in both experimental techniques and the challenging flowfield being modeled by Fulton and Edwards. [55]

Each pixel of the water vapor flux distribution accounts for the water vapor flux through that $2 \mathrm{~mm} \times 2 \mathrm{~mm}$ pixel. To calculate the total mass flow rate of the $H_{2}$ molecules, the water vapor flux multiplied by each pixel's areas are summed over the hardware boundaries of the UVaSCF. The resulting water vapor mass flow rate is then compared to the known mass flow rate of the injected $H_{2}$ to calculate the combustion efficiency. These values are tabulated in Table 4.4. For case 2, it is seen that the experimental measurement of combustion efficiency utilizing TDLAT and SPIV results in a combustion efficiency of $98.42 \%$, while the CFD calculation predicts 99.46\%. Again, this excellent agreement between experimental results and Fulton and Edward's CFD simulation of the UVaSCF places a great deal of confidence in the ability of the CFD simulation to accurately model a majority of the flow physics present at the exit of this extremely challenging flowfield. Again, the slightly higher combustion efficiency of the CFD simulations can be attributed to the Burke kinetics 
model, which results in over-predicted heat release.

TABLE 4.4: Summary of UVaSCF combustion efficiency via TDLAT \& SPIV.

\begin{tabular}{lllll}
\hline $\begin{array}{l}\text { Case } \\
\text { Number }\end{array}$ & $\begin{array}{l}\mathrm{H}_{2} \text { Equivalence } \\
\text { Ratio, } \phi\end{array}$ & $\begin{array}{l}\mathrm{H}_{2} \dot{m} \text { (Facility) } \\
\text { grams } / \text { sec }\end{array}$ & $\begin{array}{l}\mathrm{H}_{2} \mathrm{O} \dot{m} \text { (TDLAT \& SPIV) } \\
\text { grams } / \text { sec }\end{array}$ & $\begin{array}{l}\text { Combustion } \\
\text { Efficiency }\end{array}$ \\
\hline 2 (Experimental) & 0.18 (Scram-mode) & $0.974 \pm 1 \%$ & $0.959 \pm 8.6 \%$ & $98.42 \% \pm 8.7 \%$ \\
2 (CFD) & 0.18 (Scram-mode) & $0.974 \pm 1 \%$ & 0.969 & $99.46 \%$ \\
3 (Experimental) & 0.49 (Ram-mode) & $2.64 \pm 1 \%$ & $2.077 \pm 8.3 \%$ & $78.67 \% \pm 8.4 \%$ \\
\hline
\end{tabular}

The combustion efficiency is calculated from TDLAT and SPIV measurements for both the scram-mode and ram-mode, as seen in Figure 4.38. The left column is once again experimental distributions used in the combustion efficiency calculation for the scram-mode, while the right column shows experimentally measured distributions for the ram-mode. The first row shows the water vapor number densities for the two modes, measured by TDLAT. It is evident once again that the amount of water produced in the ram-mode case is significantly higher than is produced in the scrammode case. The axial velocities for both modes of operation are seen in the second row of Figure 4.38. The scram-mode case shows high-speed flow concentrated mainly in half of the duct opposite the fuel injector, with maximum velocities near $630 \mathrm{~m} / \mathrm{s}$ and a mean of $265 \mathrm{~m} / \mathrm{s}$. The ram-mode case is seen to have high-speed flow across nearly the entire duct, with maximum velocities near $785 \mathrm{~m} / \mathrm{s}$ and a mean of $375 \mathrm{~m} / \mathrm{s}$. The water vapor fluxes through each $2 \mathrm{~mm} \times 2 \mathrm{~mm}$ pixel are seen in the third row of Figure 4.38. It is notable how much larger the water vapor flux is for the ram-mode case than the scram-mode case, due to both its higher water vapor number density and axial velocity (both peak and average).

To compute the combustion efficiency from the experimentally measured water vapor number density distributions and axial velocity distributions, acquired by TDLAT and SPIV respectively, again each pixel in the water vapor flux distribution is multiplied by its area and summed over the UVaSCF hardware boundaries. The resulting water vapor mass flow rates are compared to the mass flow rate of injected $H_{2}$ to obtain the combustion efficiency. The resulting combustion efficiency for both the 


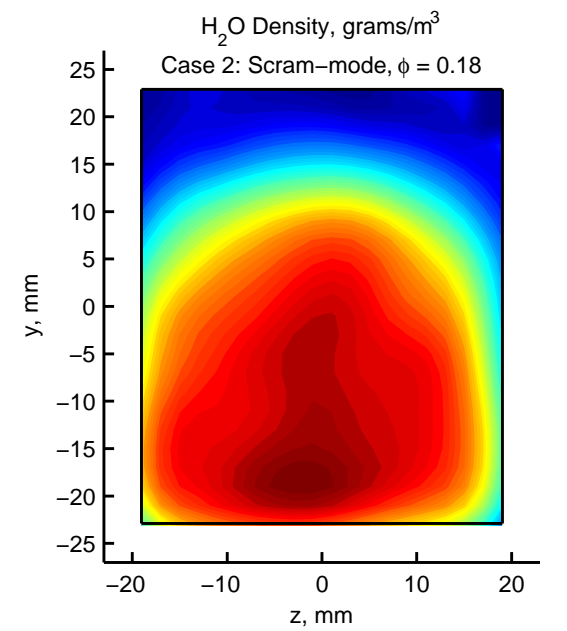

(a)

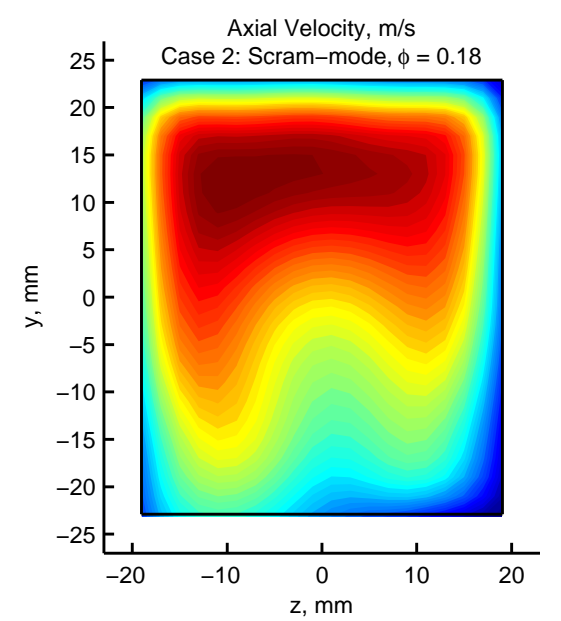

(c)

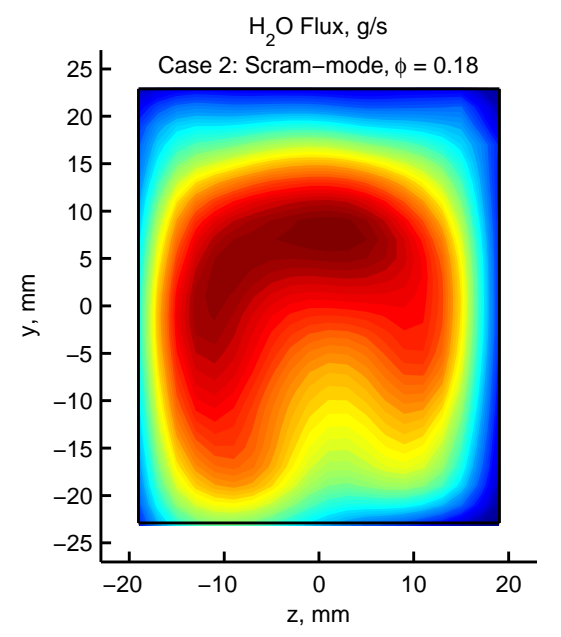

(e)
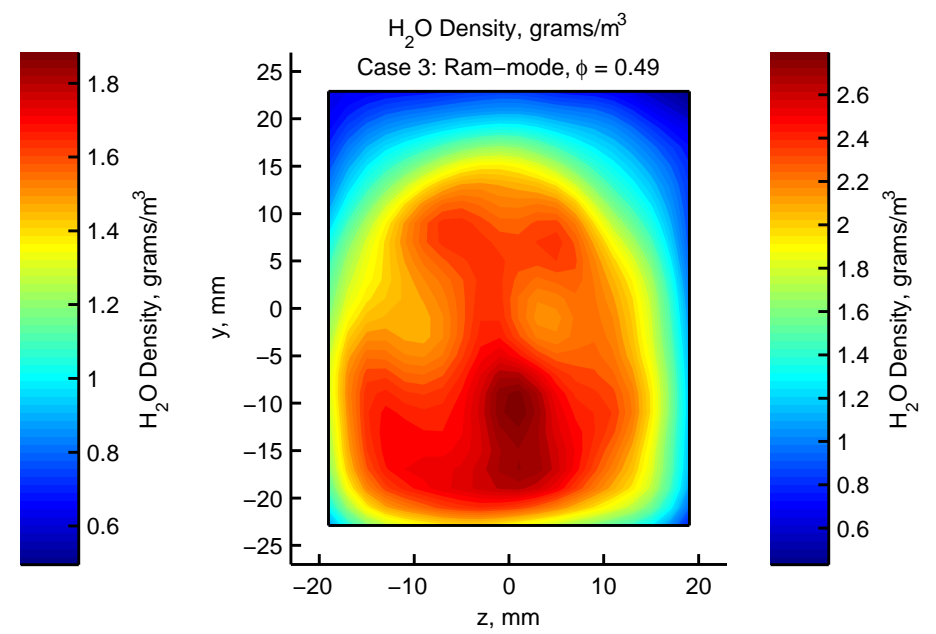

(b)
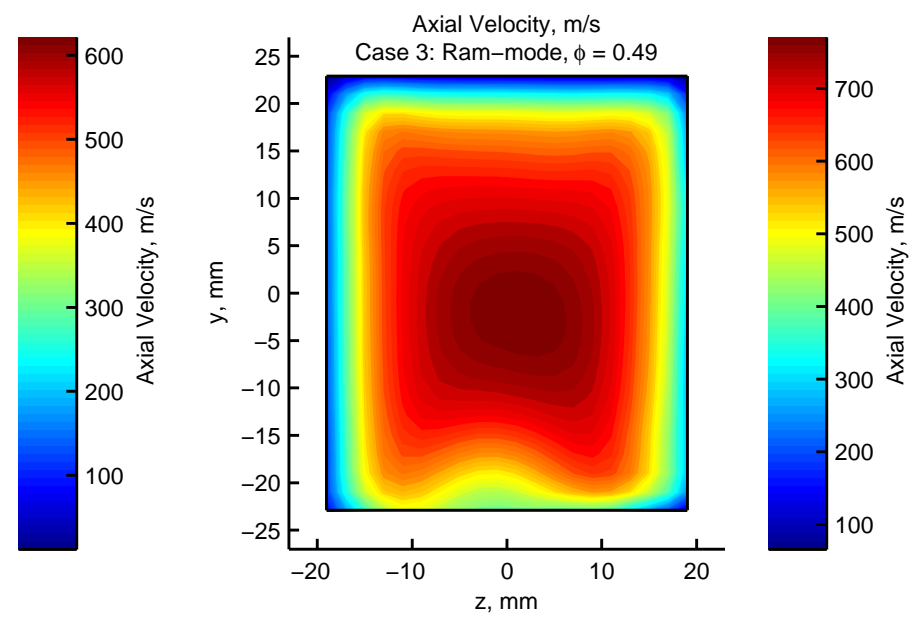

(d)
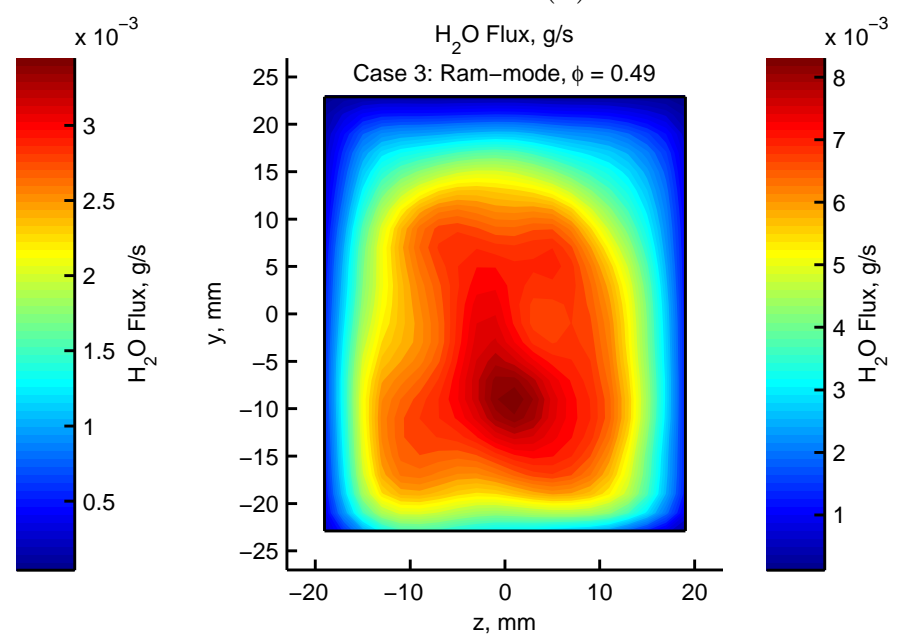

(f)

FIGURE 4.38: Water vapor number density from TDLAT measurement, axial velocity from SPIV measurement [60], and water vapor flux for the scram-mode (first column) and ram-mode (second column). 
scram-mode and ram-mode conditions tested in the UVaSCF are shown in Table 4.4. It is seen that the combustion efficiency of the ram-mode operating point tested is $78.67 \%$, significantly lower than was measured for the scram-mode operating point. This is due to increased $H_{2}$ fuel mass flow rate of $\sim 2.7$, while the average water vapor density increased by 1.4 and the average axial velocity also increased by 1.4 , as compared to the scram-mode.

The lower combustion efficiency observed for the ram-mode operating point is supported by CARS measurements of the UVaSCF for both operating points. Measurements of $\mathrm{H}_{2}$ mole fraction at a plane at $\mathrm{x} / \mathrm{H}=56.1$ show distributions of nearly $0 \% \mathrm{H}_{2}$ for the scram-mode, but approximately 8-10\% $\mathrm{H}_{2}$ for the ram-mode. [58] This indicates that a portion of the $H_{2}$ fuel has still not combusted at a plane which is located in the extender section of the UVaSCF. This presence of un-combusted fuel would decrease the combustion efficiency, as is measured by the TDLAT \& SPIV combustion efficiency calculation. Also, SPIV measurements have indicated that the ramp is less efficient at mixing the fuel and air during the ram-mode of operation than the scram-mode, due to the weaker vortices generated by the subsonic flow over the ramp. This decrease in fuel-air mixing also supports the findings of the TDLAT \& SPIV combustion efficiency calculation. 


\section{Chapter 5}

\section{NASA Langley Results}

\subsection{Introduction and Motivation}

Successful implementation of the TDLAT technique to the UVaSCF has prompted an extension of the technique to a more challenging experiment, with regard to experimental parameters. Of particular interest to scramjet ground testing is the ability to "scale" up in size, specifically in the magnitude of air mass flow rate and size of the vehicle or flowpath. As reported herein, TDLAT has been reconfigured to make spatially resolved temperature and species concentration measurements in the NASA Langley Direct-Connect Supersonic Combustion Test Facility (DCSCTF). The DCSCTF provides ten times the flow rate of the UVaSCF, and as such the complexity of this facility provided significant challenges compared to the UVaSCF. There were three inherent challenges with applying the TDLAT technique to the DCSCTF that were encountered and managed:

1. Larger test section area. The area of the DCSCTF exit plane is 5.5" $\times 5.2 "$ compared to the UVaSCFs $1.5 " \times 1.8 "$ exit plane.

2. Decreased test duration. Typical DCSCTF test times range between 30 and 50 seconds compared to hours of uninterrupted testing in the UVaSCF.

3. Harsh and noisy conditions at the DCSCTF exit plane. 
The first two problems listed were anticipated and considered in the preliminary phases of this research. The third problem was encountered during active testing and was resolved in the midst of testing.

\section{$5.2 \quad$ NASA Langley Direct-Connect Supersonic Combustion Test Facility}

The NASA Langley DCSCTF Durable Combustor Rig (DCR) is capable of testing ramjet and scramjet combustor models at conditions simulating flight from Mach 4 to Mach 8. A Mach 5 flight-enthalpy has been simulated for this research. Hydrogen combustion with oxygen-enriched air is used to heat the test gas to achieve the required conditions, known as vitiated heating. The facility can operate with a variety of nozzles and (sc)ramjet models. The DCSCTF is ideal for research in mixing, ignition, flameholding, and combustion characteristics. [62] Figure 5.1 shows a photograph of the DCSCTF with the TDLAT measurement system mounted to it. The DCSCTF is a blow-down facility, meaning that test air is supplied from a high-pressure bottle field and is either exhausted to atmospheric conditions or to a vacuum sphere. For the TDLAT experiments the DCSCTF test gas was exhausted to atmosphere and then was vented outside of the test facility. Flow is from left to right in Figure 5.1.

The TDLAT hardware is mounted to the exit plane of the DCSCTF inside the test cell, seen in Figure 5.1. During operation and between tests, the test cell remains closed. Therefore, although the TDLAT hardware is located inside the test cell, the TDLAT computer (runs data acquisition and motion control software) and the diode lasers are located in an adjacent room. The power cables, data cables, and optical fibers are fed into the test cell through a small hole in the wall as seen in Figure 5.2. This allows the user to actively control the TDLAT equipment remotely. 


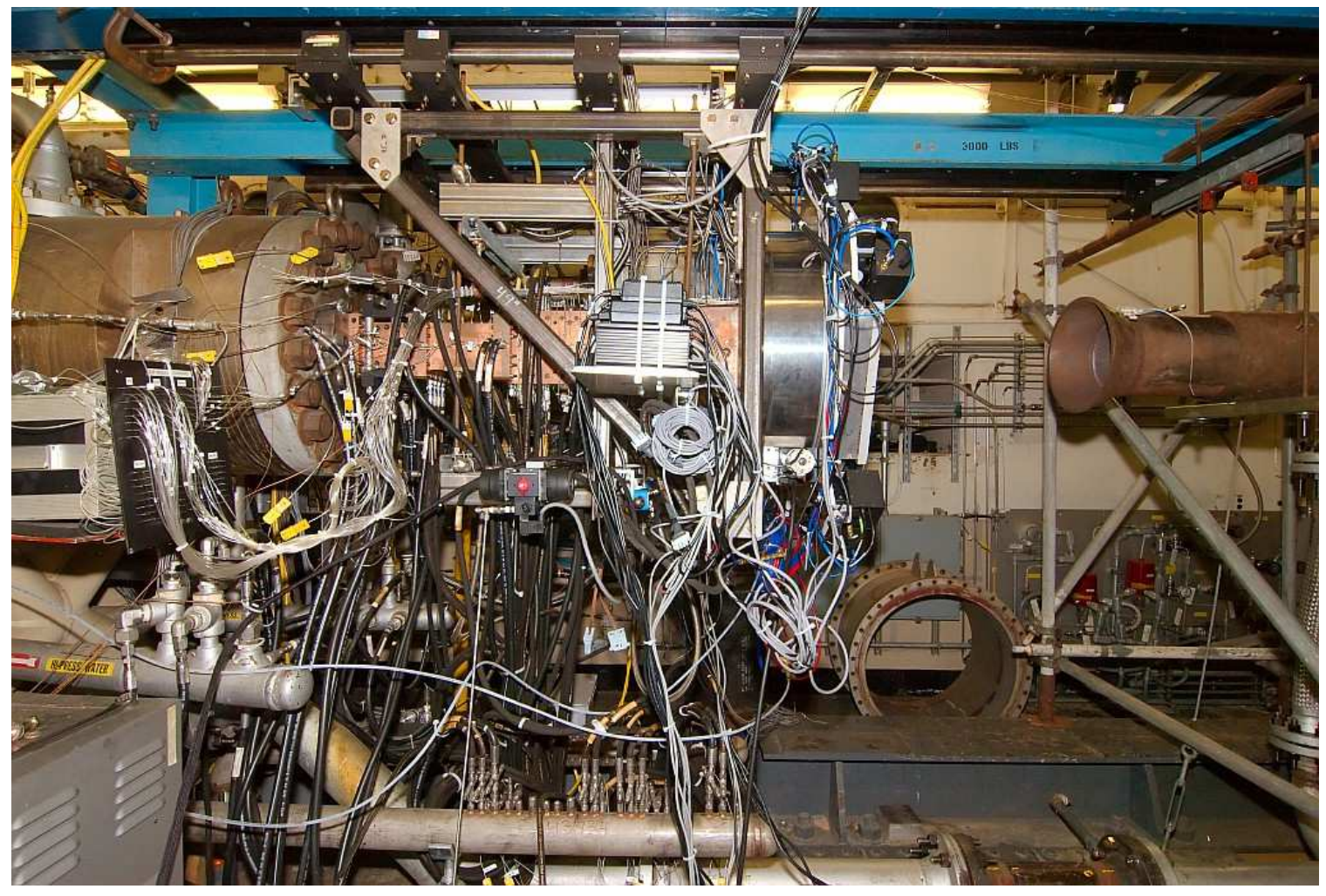

Figure 5.1: NASA Langley Direct-Connect Supersonic Combustion Test Facility with TDLAT system installed.

\section{DIRECT-CONNECT SUPERSONIC COMBUSTION TEST FACILITY} Outer door Top view B1221-D/R124

Outer door

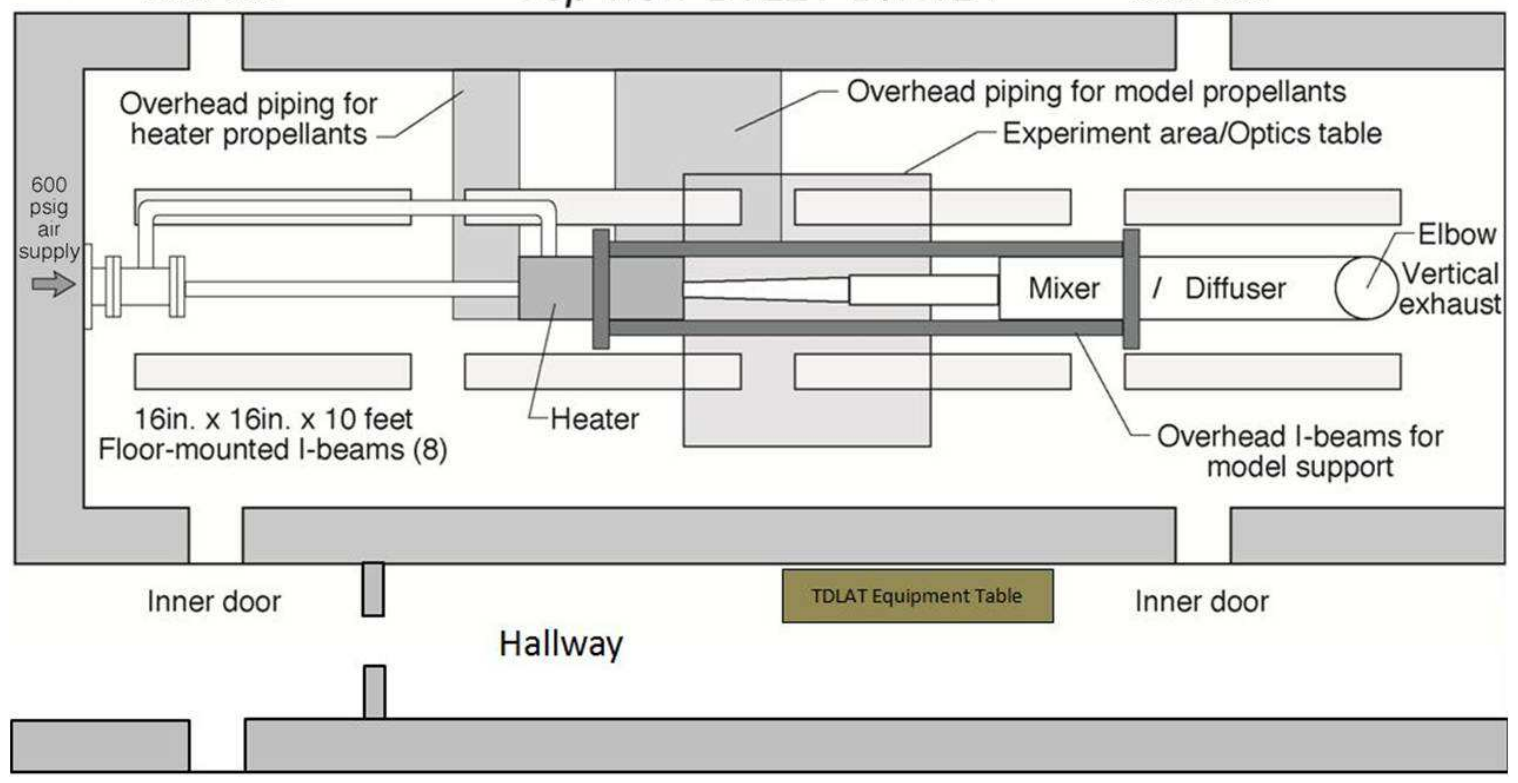

FiguRE 5.2: Schematic of DCSCTF and location of TDLAT equipment. 
As mentioned previously, there were two major facility challenges faced when applying the TDLAT system to the DCSCTF: the larger test section area and the decreased test duration. First, the larger test section area called for hardware modifications. The Newport rotational ring used for the tests conducted on the UVaSCF measured approximately 7" internal diameter, while the custom Newport rotational ring used for the DCSCTF measured approximately 19" internal diameter, see Figure 5.3. Also noteworthy is that the UVaSCF has a vertical test section which allowed the TDLAT rig to be mounted horizontally, with its axis of rotation perpendicular to the ground. The DCSCTF has a horizontal test section, requiring the TDLAT rig to be mounted vertically with its axis of rotation parallel to the ground.

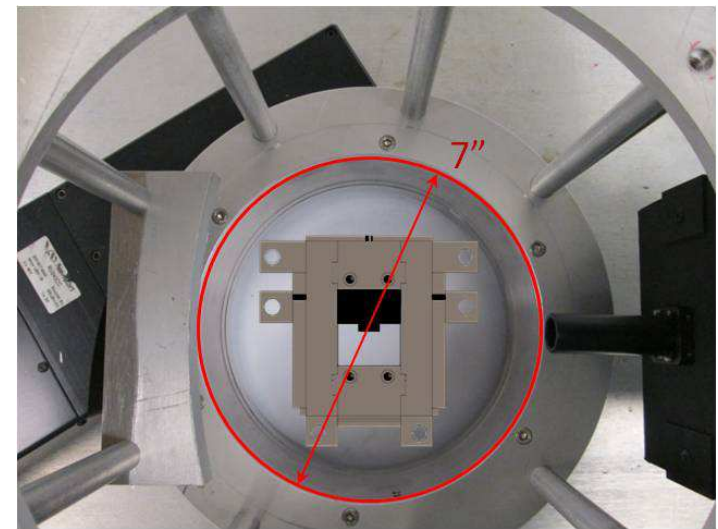

(a)

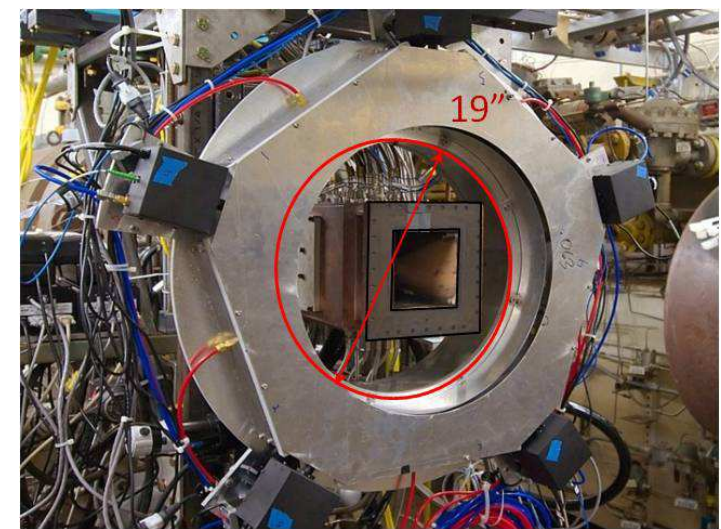

(b)

FigURE 5.3: TDLAT system used for measurements on (a) the UVaSCF, and (b) the NASA Langley DCSCTF.

The second challenge, the short test duration of the DCSCTF, called for both software and hardware modifications. The 50 second maximum test time is limited by the temperature of the un-cooled copper hardware on the DCSCTF rig. This required streamlining of the motion control and data acquisition software used in the UVaSCF. Various portions of the LabVIEW 8.6 code were condensed or removed altogether if unnecessary. Much of the extraneous code was for data communication between the PC and the motion controllers and was able to be replaced with simple TTL (transistor-transistor logic) signals. The shorter test duration also motivated 
the decision to increase the number of TED boxes from one to five, creating the multi-box configuration. This reduced the required test duration by the same factor. Figure 5.4 shows the multi-box TDLAT configuration.

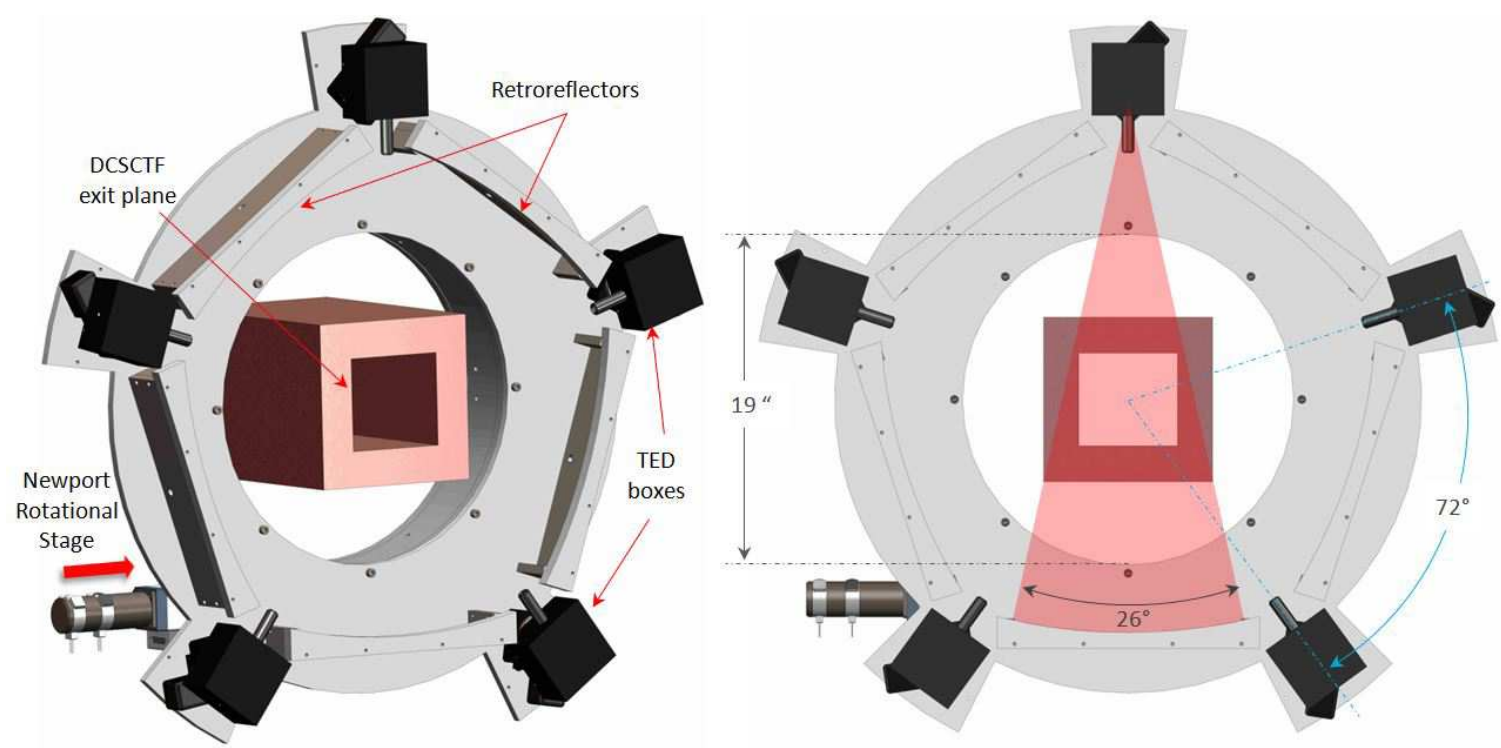

FIgURE 5.4: SolidWorks model of the multi-box TDLAT configuration for use on the DCSCTF.

As seen in Figure 5.4, the five TED boxes and retroreflector hardware are mounted on a Newport RTM660 rotational ring. This allows the assembly to rotate $360^{\circ}$ around the DCSCTF. However, due to the angular symmetry of the five TED boxes, the plate needs only to rotate $72^{\circ}$ for complete imaging. Attached to the adapter plate are five Velmex B5990TS rotational stages and five TED 2.0 boxes. The Velmex rotational stages allow each TED box to rotate and create a fanbeam. As seen in Figure 5.4, $26^{\circ}$ of rotation was needed to ensure the fanbeam encompasses the DCSCTF at the exit plane.

Motion and laser control have been optimized to allow for data collection during the short test duration. Each fan is composed of 21 rays which are incremented by $1.3^{\circ}$, consequently scanning a total of $26^{\circ}$. Each fan is separated by $9^{\circ}$, requiring a total of 8 data sets (each with 5 fans) to complete the database. A complete uninterrupted database requires between 6 and 7 minutes of test time, and thus the testing must be segmented. One data set, consisting of 5 fans of data, was collected 
during each tunnel operation. The start point of the fans during next test is then offset $9^{\circ}$ from the previous tunnel run. This requires 8 tunnel runs for a complete database, which is well within the test capabilities of the DCSCTF on a single day. This is a significant improvement in the test time required for the experiment - from $2+$ hours to 7 minutes.

The noisy environment of the DCSCTF created the need for several LOS measurements to be collected at each location and averaged. Figure 5.5 shows sample data from a measurement near the center of the test plane. For the TDLAT measurements of the DCSCTF, 150 individual LOS measurements are collected and averaged for each laser and at each location. The data is acquired at 100,000 samples/sec, with 150,000 samples/channel. Therefore, 1.5 seconds is spent collecting the data at each location. A sample LOS is shown, with 150 single-scan LOS and the resulting mean absorption signal.

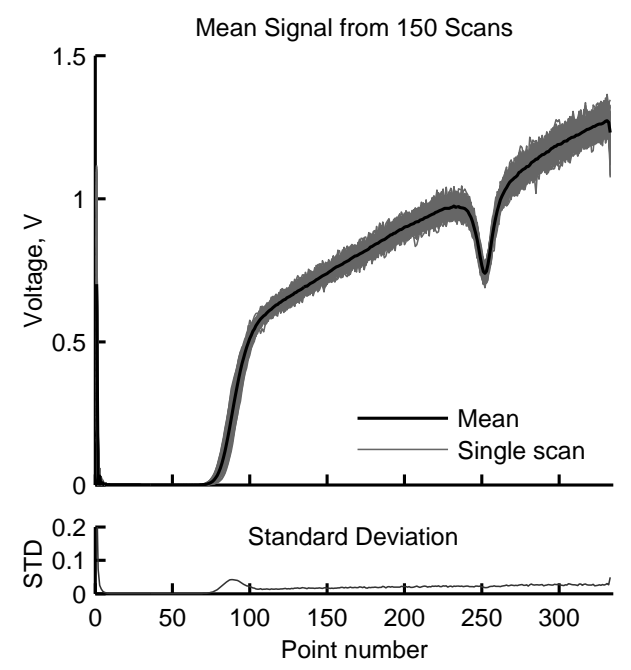

FIgURE 5.5: Single-scan and averaged LOS sample data from DCSCTF TDLAT.

The TDLAT experiments performed at the NASA Langley DCSCTF DCR fulfill Research Objectives I and IV. Successful implementation of the TDLAT technique to NASA Langley's DCR was significant due to the advancement of TDLAT's Technical Readiness Level (TRL) which is a 1-9 scale. Previously, the TDLAT system was at a TRL level 4, which NASA defines as "component and/or breadboard validation 
in laboratory environment". Through this research, the TDLAT technique has been advanced to a TRL level 6, which NASA defines as a "system/subsystem model or prototype demonstration in a relevant environment". This advancement of the technique brings us even closer to a successful "flight proven" system. The successful scaling of the TDLAT measurement system, as demonstrated by measurements on the DCSCTF, is extremely relevant and significant as research moves toward larger ground test facilities for scramjet development. The increase in size of the test facilities often prevents the use of thrust stands to characterize the performance of the engines, necessitating the use of other diagnostics such as TDLAT. As was a motive for the UVaSCF TDLAT measurements, the TDLAT measurements acquired at the DCSCTF will also be utilized for comparisons between experimental data and CFD calculations performed by Gaffney at NASA Langley Research Center's Hypersonic Airbreathing Propulsion Branch. [63]

\subsection{Experimental Parameters}

All data was collected for the TDLAT experiment at NASA Langley's DCSCTF DCR in August 2012. Combustion measurements utilizing $H_{2}$ fuel at a fuel-to-air equivalence ratio of $\phi=0.5$ were collected at a vitiated facility condition simulating flight at Mach 5. Due to its sensitive nature, the combustor geometry of the DCSCTF utilized for the TDLAT experiments will not be presented or discussed. As mentioned previously, a complete data set was captured with eight tunnel runs, resulting in a data set with 40 fans and $9^{\circ}$ between each fan. This created a dense measurement within the DCSCTF exit area. Table 5.1 summarizes the Mach 5 test conditions. 
TABLE 5.1: Test conditions during TDLAT measurements on DCSCTF.

\begin{tabular}{lllllllll}
\hline $\begin{array}{l}\text { Run } \\
\text { Number }\end{array}$ & $\begin{array}{l}\text { Cardinal } \\
\text { Angle, }\end{array}$ & Date & $\begin{array}{l}\text { Avg. Period, } \\
\text { seconds }\end{array}$ & $\begin{array}{l}P_{0}, \\
\text { psia }\end{array}$ & $\begin{array}{l}T_{0}, \\
\text { R }\end{array}$ & $\begin{array}{l}H_{T 0}, \\
\text { BTU } / l b_{m}\end{array}$ & $\begin{array}{l}O_{2}, \\
\%\end{array}$ & $\phi$ \\
\hline 89 & 36 & $8 / 16 / 2012$ & $34.0-44.0$ & 96.2 & 2113 & 578.2 & 20.82 & 0.555 \\
90 & 0 & $8 / 16 / 2012$ & $36.0-46.0$ & 96.0 & 2125 & 582.2 & 20.91 & 0.549 \\
91 & 18 & $8 / 16 / 2012$ & $39.0-49.0$ & 96.6 & 2132 & 583.9 & 20.98 & 0.562 \\
92 & 54 & $8 / 16 / 2012$ & $40.0-50.0$ & 96.6 & 2131 & 583.8 & 21.14 & 0.526 \\
93 & 9 & $8 / 16 / 2012$ & $38.5-48.5$ & 96.4 & 2132 & 584.2 & 20.86 & 0.552 \\
94 & 27 & $8 / 16 / 2012$ & $39.0-49.0$ & 96.8 & 2162 & 593.9 & 20.93 & 0.510 \\
95 & 45 & $8 / 16 / 2012$ & $40.0-50.0$ & 97.1 & 2137 & 585.9 & 20.91 & 0.533 \\
96 & 63 & $8 / 16 / 2012$ & $40.0-50.0$ & 97.1 & 2142 & 587.7 & 20.85 & 0.545 \\
\hline
\end{tabular}

\subsection{Case 4: NASA Langley DCSCTF Results}

The measured integrated absorbance for each of the four absorption features, formed by combining the measurements collected during eight tunnel runs, are shown in Figure 5.6. The left column shows the raw measured data, while the right column shows the data after minimal filtering. The magnitude of the absorbance for all four lasers is significantly higher than in the previous test cases, mostly due to the longer path length required to traverse the DCSCTF exit plane flowpath.

The integrated LOS temperatures for each of these 840 LOS can then be calculated using the Boltzmann plot analysis. Again, this integrated LOS temperature sinogram is useful mainly for preliminary inspection of the measurements and to obtain the general temperature range of the measured test gas. Shown in Figure 5.7, maximum LOS temperatures of $1,400 \mathrm{~K}$ are observed, with a mean of $\sim 1,200 \mathrm{~K}$ throughout the core of the sinogram (rays 7-15). The magnitude of this sinogram is most similar to that of case 3, which saw a maximum LOS temperature of 1,660 K and maximum reconstructed temperatures near $2,170 \mathrm{~K}$. 


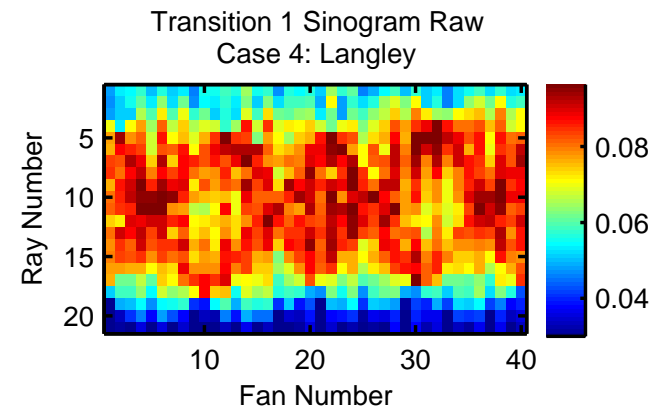

(a)

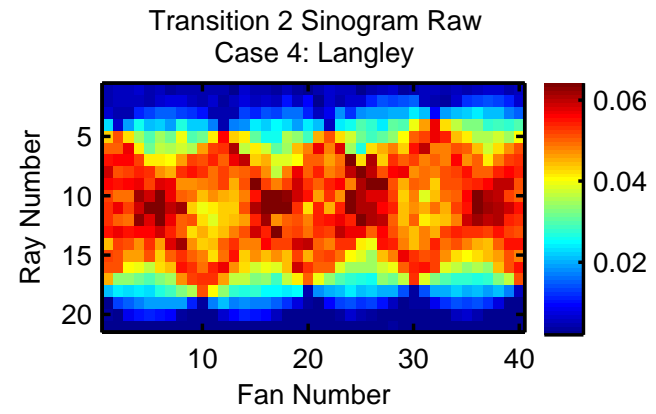

(c)

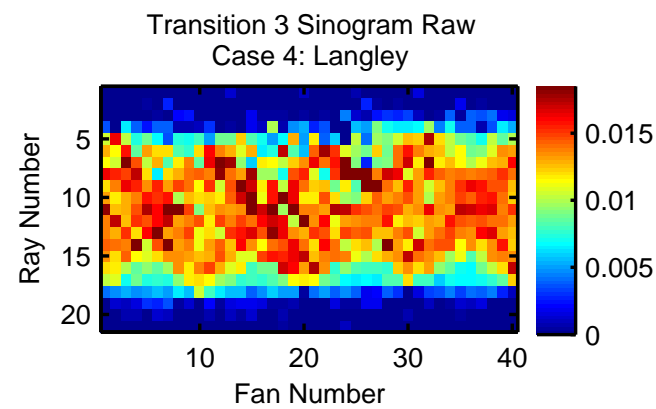

(e)

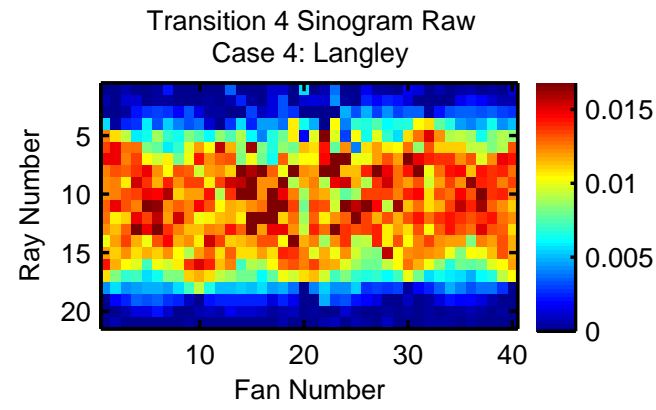

(g)

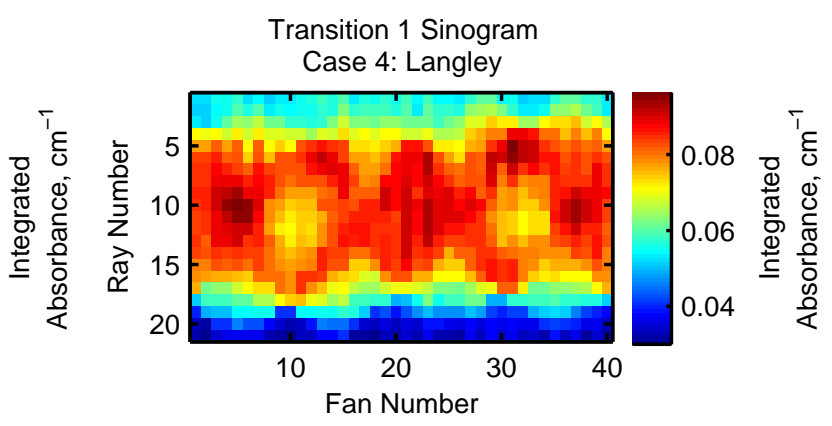

(b)

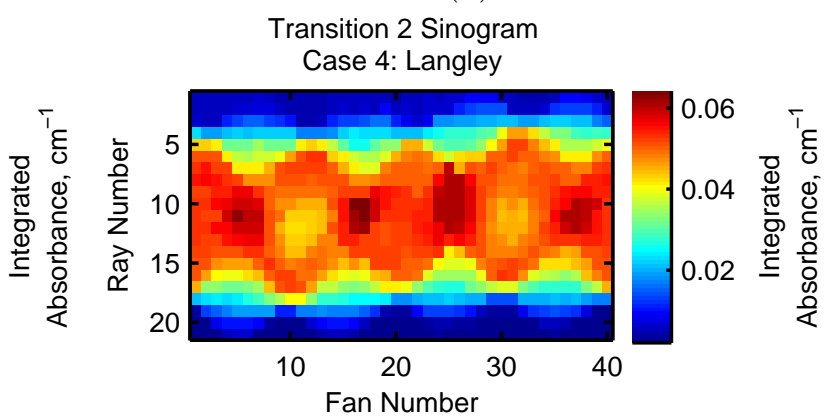

(d)

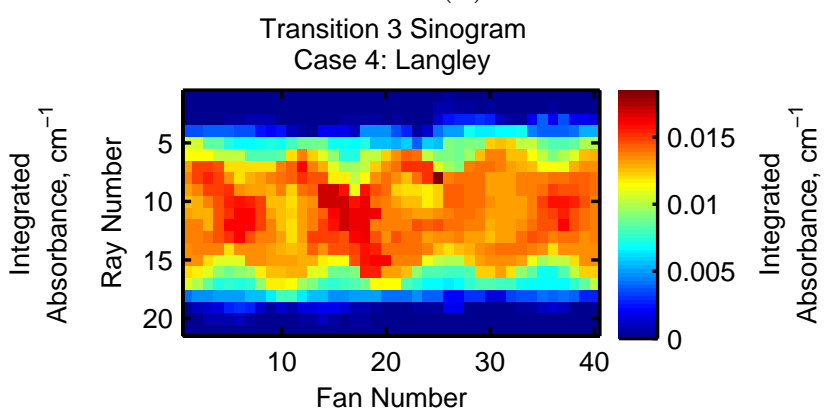

(f)

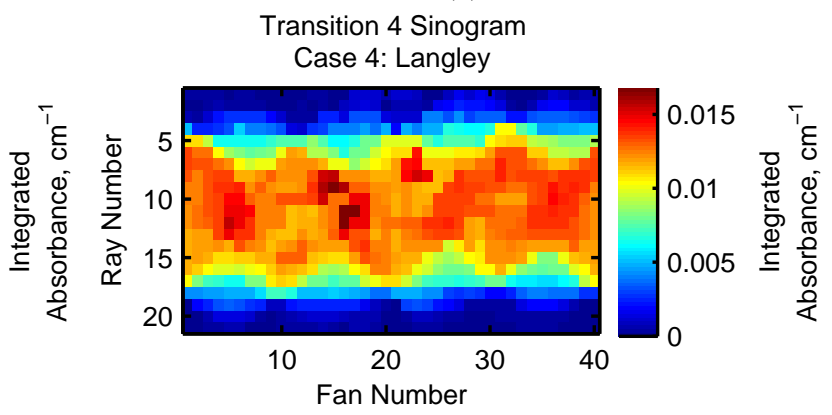

(h)

FiguRE 5.6: Integrated absorbance sinograms at the exit plane of the DCSCTF for case 4. Sinograms are formed by combining the measurements collected during eight tunnel runs. The first column contains the raw sinograms of TDLAT measured integrated absorbance for each of the four absorption features (KB1-KB4) and the second column contains the corresponding filtered sinograms. 


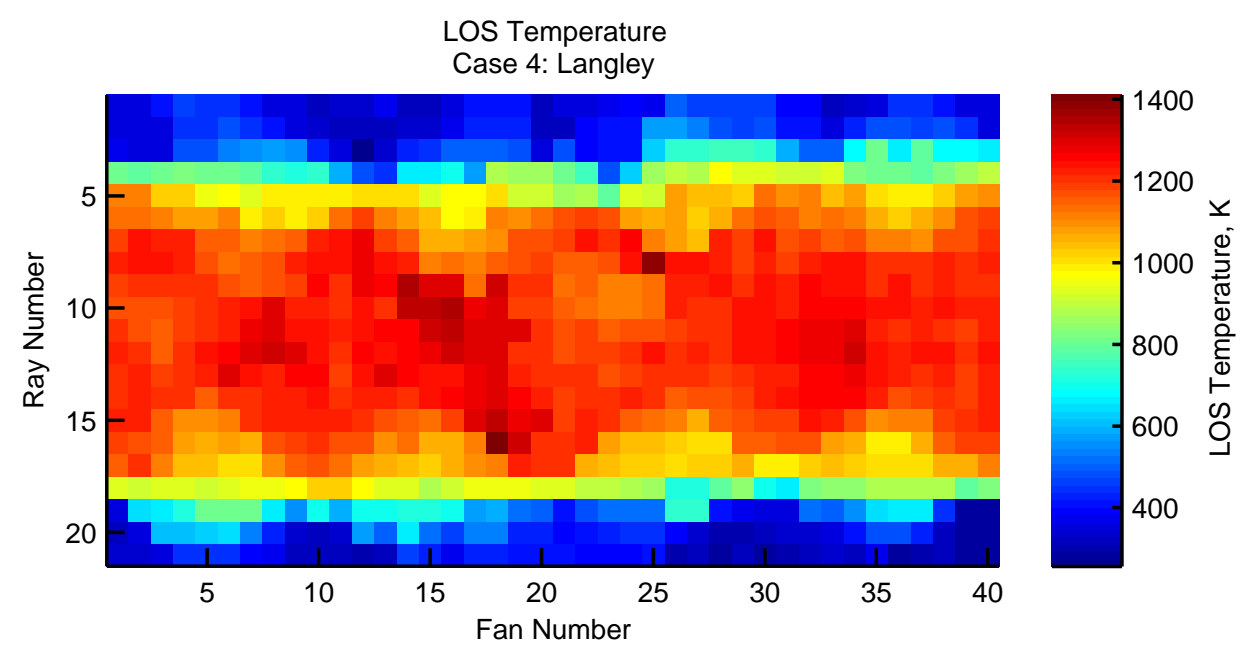

FiguRE 5.7: LOS temperatures calculated for case 4 TDLAT measurements.

The measured integrated absorbance sinograms seen in Figure 5.6 were reconstructed using the ML-EM algorithm and the resulting absorbance distributions are seen in Figure 5.8. The reconstruction of the KB1 transition is quite distinct and has a distribution unlike any other reconstructions within the scope of this work. A significant portion of the KB1 absorbance is shown concentrated along the upper and lower walls of the DCSCTF. A "valley" between these two ridges of high absorbance is also observed, with two depressions, located near $\mathrm{z}= \pm 30 \mathrm{~mm}$. The KB2 transition shows a similar distribution, but the difference between the absorbance at $\mathrm{y}= \pm 60$ $\mathrm{mm}$ and the absorbance near $\mathrm{y}=0 \mathrm{~mm}$ is not as severe as seen for KB1. Recalling the equation for two-line thermometry, Equation 2.10, temperature is a function of $1 / \ln \left(A_{1} / A_{2}\right) . A_{1} / A_{2}$ will be lower near the center of the DCSCTF than along the top and bottom edges, resulting in higher central temperatures than observed on the upper and lower edges. The KB3 and KB4 absorbance distributions do not show this striated pattern quite as distinctly as the other absorption features, though they do each possess a region of low absorbance near $(0,0) \mathrm{mm}$. 


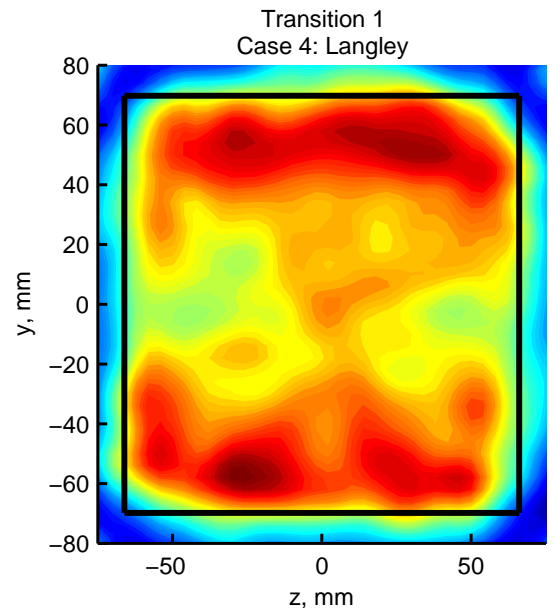

(a)

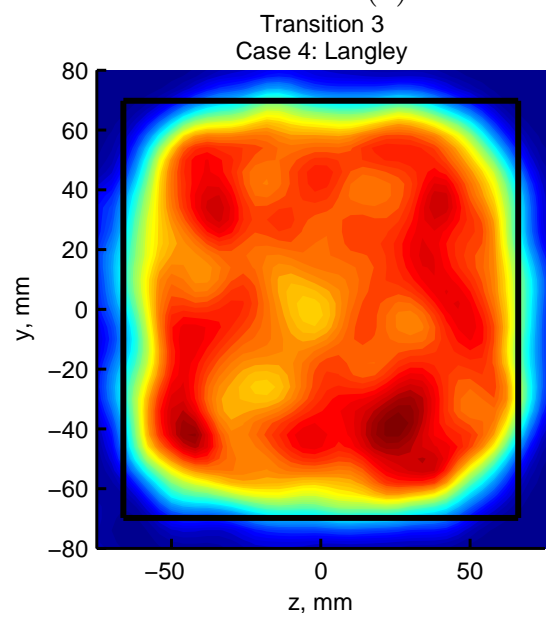

(c)
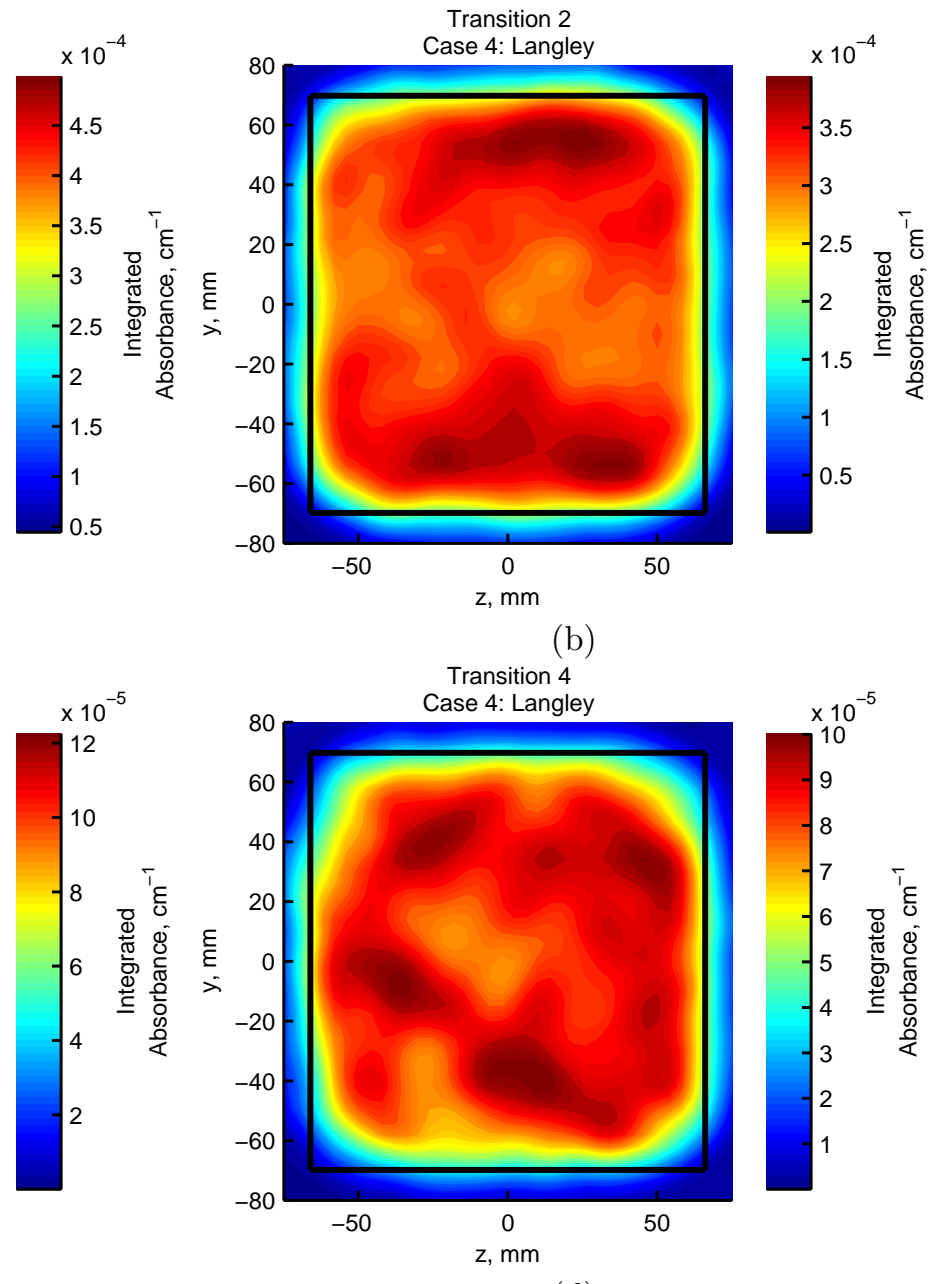

(d)

Figure 5.8: Reconstructed integrated absorbance at the exit plane of the NASA Langley DCSCTF for case 4, for (a) KB1, (b) KB2, (c) KB3, and (d) KB4. 
Equation 2.10 can be utilized for each combination of absorption features, shown in Figure 5.9. As was speculated, each combination of absorption features results in a temperature distribution which contains high temperature regions near the center of the duct. It is also apparent that the high temperature regions are focused into two areas, somewhat symmetrically distributed in z.

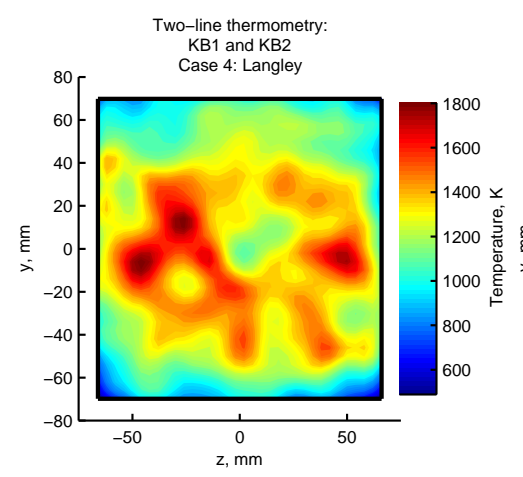

(a)

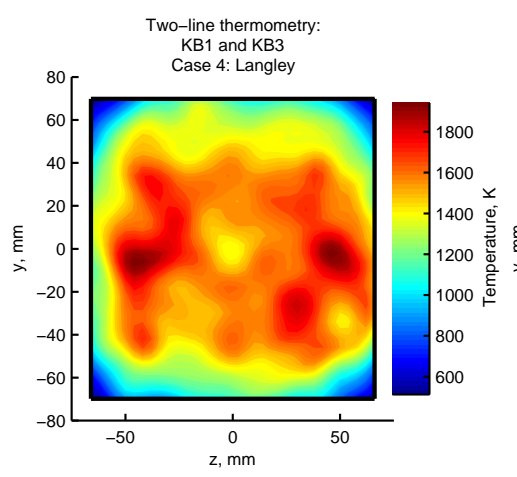

(b)

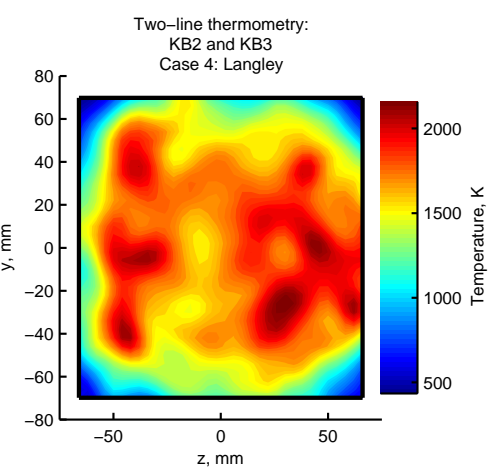

(c)

Figure 5.9: Two-line thermometry calculation via Equation 2.10 at the exit plane of the NASA Langley DCSCTF for case 4, for line-pairs (a) KB1 and KB2, (b) KB1 and KB3, and (c) KB2 and KB3.

To utilize the contributions of all three transitions simultaneously, (KB4 will again be discarded for this phase) the Boltzmann plot method is employed at each spatial location. A distribution of the RMSE of the linear fit at each pixel is shown in Figure 5.10 , showing excellent collinearity of the reconstructed integrated absorbance values.

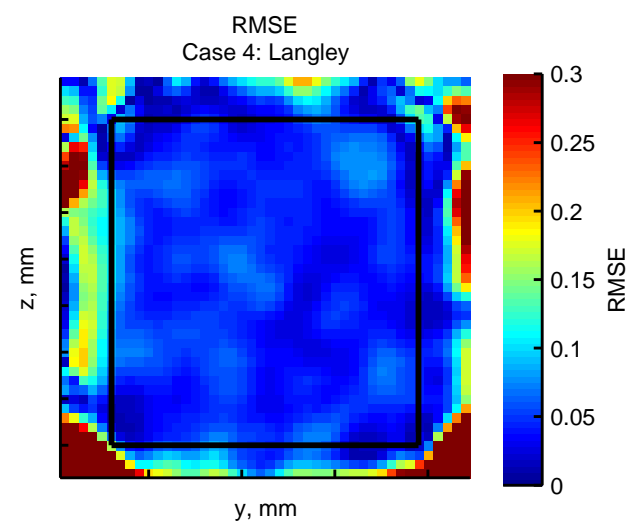

Figure 5.10: Root-mean square error for Boltzmann plot at the exit plane of the NASA Langley DCSCTF for case 4. 
Figure 5.11 shows the resulting temperature, water vapor number density, and water vapor mole fraction distributions at the exit plane of the NASA Langley DCSCTF at the test conditions described in Table 5.1. Two lobes of high temperatures are seen, near $\mathrm{z}= \pm 50 \mathrm{~mm}$. These regions of high temperature are concentrated near $\mathrm{y}=0 \mathrm{~mm}$, but stretch vertically across the duct spanning $65-75 \%$ of the space. The water vapor number density distribution shows nearly constant values of $\mathrm{N}_{\mathrm{H}_{2} \mathrm{O}}$ across the duct, with several small local maxima observed. A depression near the center of the duct is observed in all of the reconstructed distributions. The water vapor mole fraction distribution shows the same two lobes observed in the temperature distribution, with maximum water vapor mole fractions near 34\%. The average water vapor mole fraction across the DCSCTF duct is $22 \%$. Note that the freestream is expected to contain approximately $12 \% \mathrm{H}_{2} \mathrm{O}$ by mole, due to the vitiated heater. [57][62] 


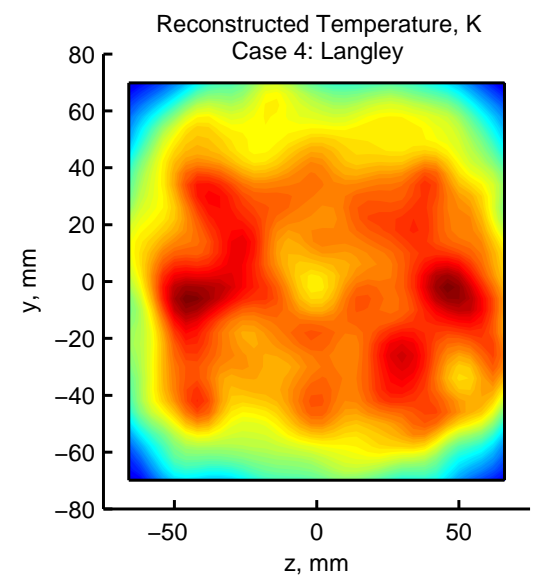

(a)

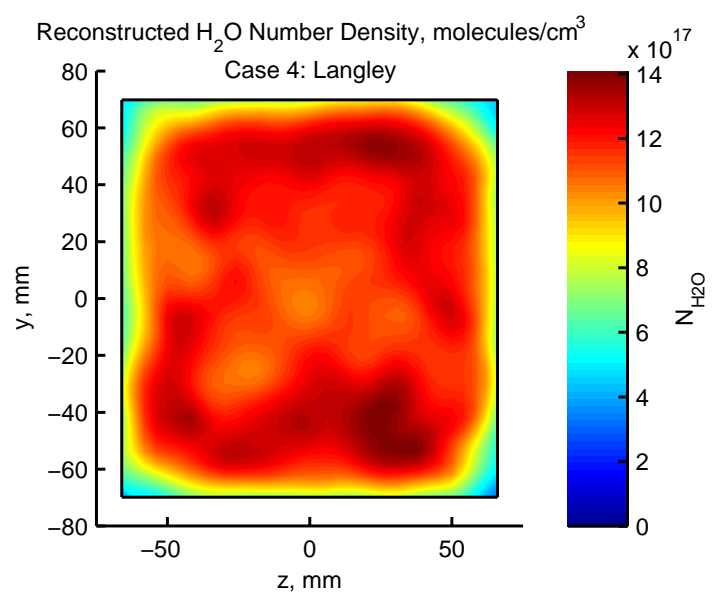

(b)

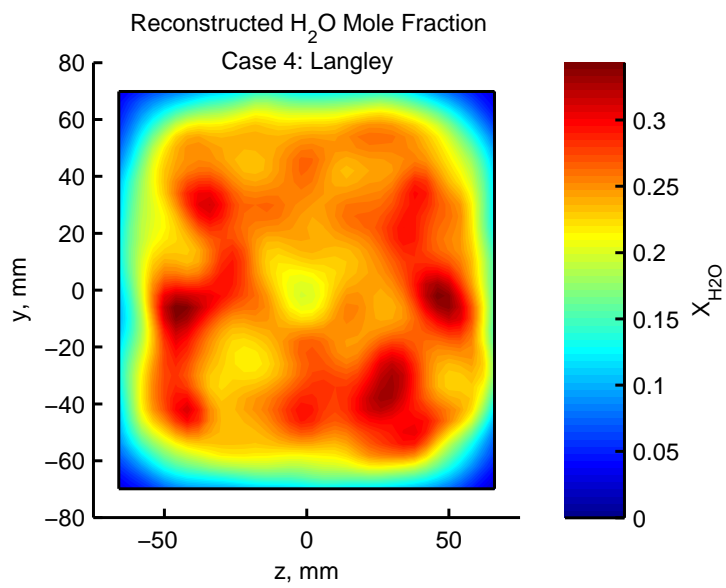

(c)

FiguRE 5.11: Final reconstructions at the exit plane of the NASA Langley DCSCTF for case 4: (a) temperature, (b) water vapor number density, and (c) water vapor mole fraction. 
These experimentally measured distributions of temperature, water vapor number density, and water vapor mole fraction can be directly compared to CFD simulations of the same DCSCTF facility conditions performed by Rick Gaffney. The CFD distributions have been interpolated onto the same grid as the TDLAT results to enable direct comparison. Side-by-side comparisons of the experimentally measured and CFD simulated distributions at the exit plane of the DCSCTF are shown in Figure 5.12. These comparisons have been plotted such that the colorbars of the experimental and CFD distributions are the same.

The first row of Figure 5.12 shows the temperature distributions. The two lobes of high-temperature discussed earlier are also evident in the CFD distribution. A third lobe is also visible, located at $(0,-20) \mathrm{mm}$, resulting in a distribution resembling a capital "M" for both the TDLAT-acquired and CFD-simulated temperature distributions. The bottom of the high-temperature "M" is seen to be near $\mathrm{y}=-45 \mathrm{~mm}$ in both distributions, while the top of the high-temperature "M" extends to only y $=40$ $\mathrm{mm}$ for the TDLAT and $\mathrm{y}=65 \mathrm{~mm}$ for the CFD. Near the top surface of the duct, at $(0,70) \mathrm{mm}$, a single small depression is visible in both the the experimental and CFD temperature distributions. Similarly, several depressions are seen along the bottom surface of the duct at $(0,-70) \mathrm{mm}$ for both the TDLAT and simulated distributions. Both methods also agree well in the corners of the duct, showing pockets of lowtemperature gas. Overall, the CFD predicts higher temperatures than the TDLAT experiment measured. However, the shape of the distributions are quite similar.

The second row of Figure 5.12 shows the resulting water vapor number density distributions for both TDLAT and CFD. Though both distributions show a significant portion of the number density approximately equal to $1.3 \times 10^{18}$ molecules $/ \mathrm{cm}^{3}$, it is interesting that the two distributions diverge in all other locations across the duct. For example, the CFD measurement predicts higher $N_{H_{2} \mathrm{O}}$ toward the center of the duct, while the TDLAT measured lower $N_{\mathrm{H}_{2} \mathrm{O}}$ values at the center of the duct. Similarly, the CFD also predicts large values of $N_{H_{2} O}$ near the walls of the duct, while the 
TDLAT measured low $\mathrm{N}_{\mathrm{H}_{2} \mathrm{O}}$ values.

The third row of Figure 5.12 presents the water vapor mole fraction distributions measured by TDLAT and simulated by CFD. The "M" shape is again observed, though slightly less distinct than was seen in the temperature distributions. Maximum water vapor mole fractions near $40 \%$ are observed for both the experimental and simulated distributions. The CFD appears to over-predict the amount of water vapor in the corners of the duct, as well as along each wall. The TDLAT measures $X_{\mathrm{H}_{2} \mathrm{O}}$ $\sim 5 \%$ in the corners of the flow, and $\sim 17 \%$ along the walls, while the CFD predicts values between $22-25 \%$ around most of the DCSCTF hardware boundary. The CFD also shows the water vapor mole fraction distribution extend fully across the duct, from $\mathrm{z}=-66 \mathrm{~mm}$ to $\mathrm{z}=66 \mathrm{~mm}$, while the TDLAT measures a transitional region between the high temperature, high water vapor mole fraction core flow and the hardware boundary.

For further clarification, line plots are shown in Figure 5.13 along $\mathrm{y}=0 \mathrm{~mm}$ and $\mathrm{z}=0 \mathrm{~mm}$ for all three measured quantities. The CFD distributions are shown in black and the TDLAT measurements are shown in color. The agreement between the CFD simulation and TDLAT measurements at the center lines is moderate. This is unsurprising, as the main structure at the DCSCTF exit was seen to be off-center. Therefore, off-center profiles of temperature, water vapor number density, and water vapor mole fraction are shown in Figures 5.14, 5.15, and 5.16 respectively. Each figure shows two vertical profiles, at $\mathrm{z}=-46 \mathrm{~mm}$ and $\mathrm{z}=42 \mathrm{~mm}$, and two horizontal profiles, at $\mathrm{y}=-46 \mathrm{~mm}$ and $\mathrm{y}=30 \mathrm{~mm}$. 


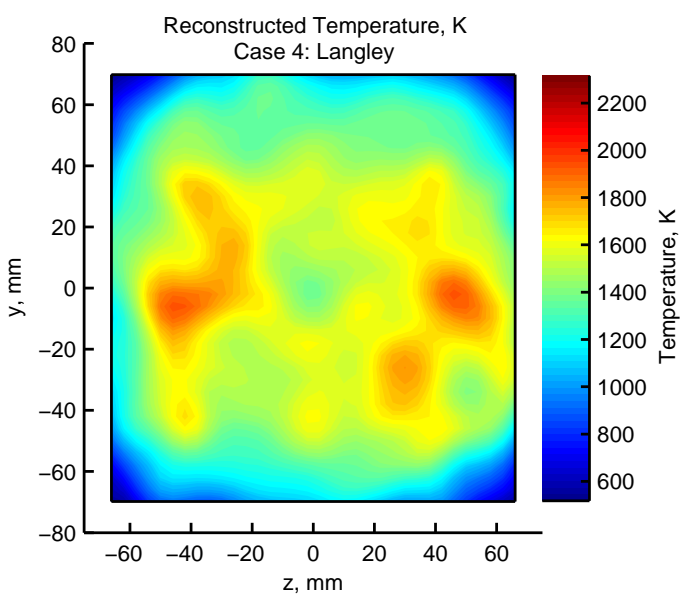

(a)

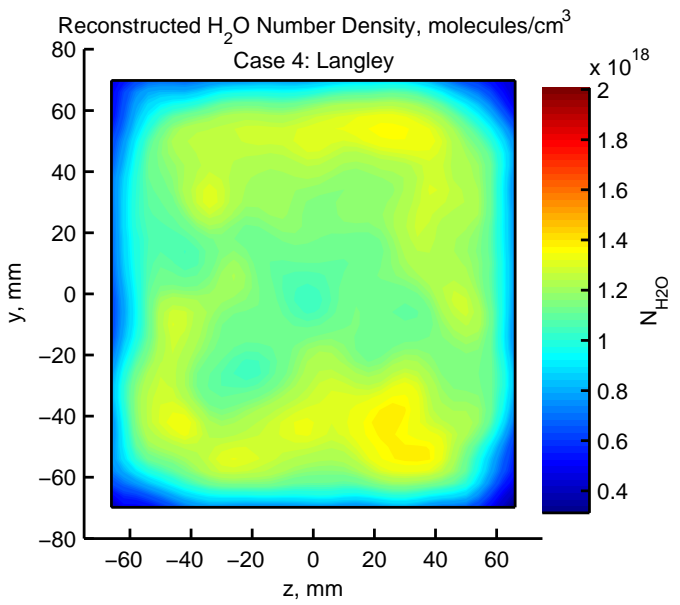

(c)

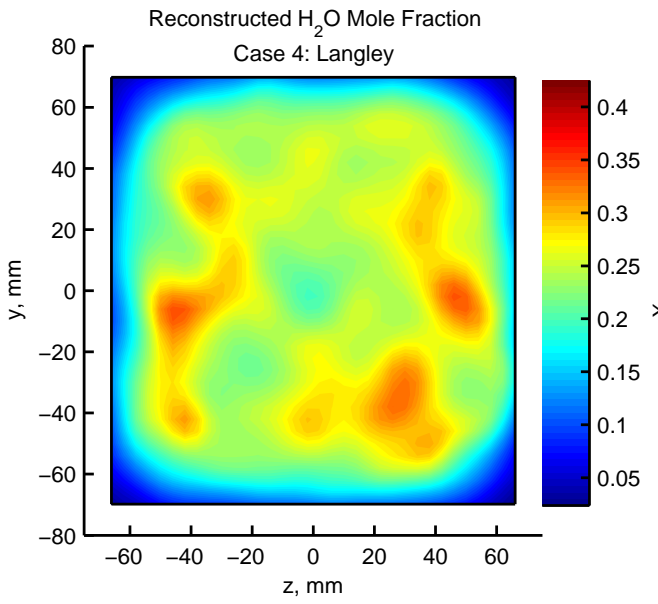

(e)

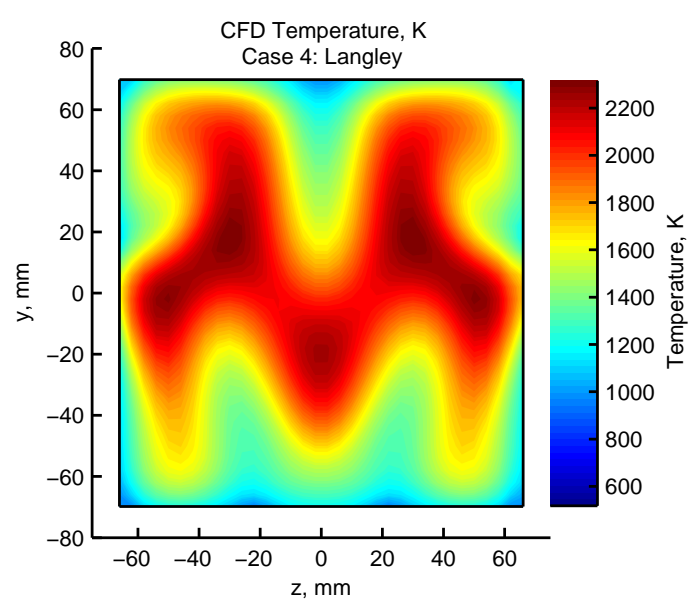

(b)

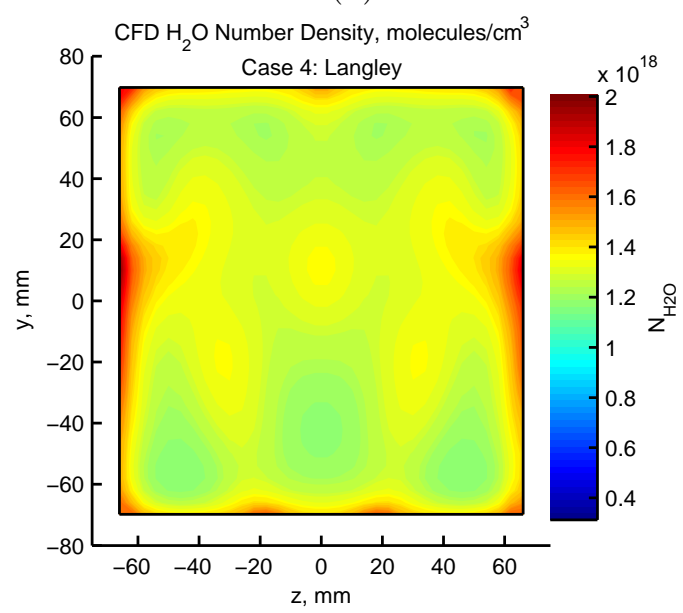

(d)

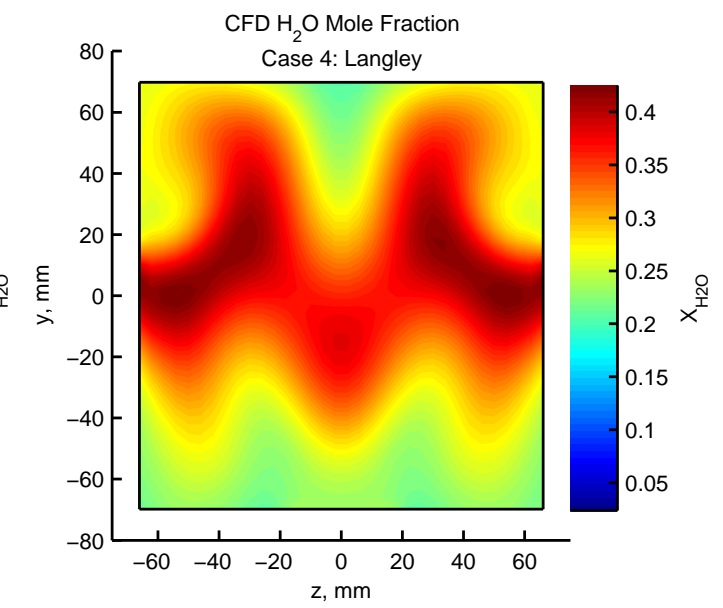

(f)

FiguRE 5.12: Side-by-side comparisons of TDLAT measurements and CFD at the exit plane of the NASA Langley DCSCTF for case 4, (a) TDLAT temperature distribution, (b) CFD temperature distribution, (c) TDLAT water vapor number density distribution, (d) CFD water vapor number density distribution, (e) TDLAT water vapor mole fraction distribution, and (f) CFD water vapor mole fraction distribution. 


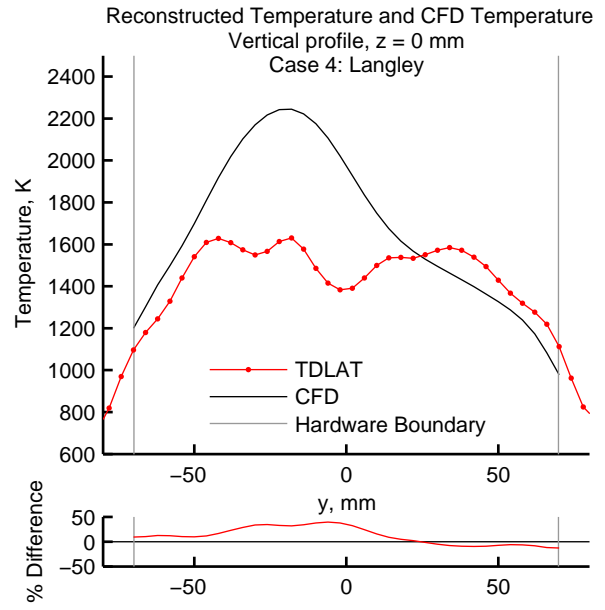

(a)

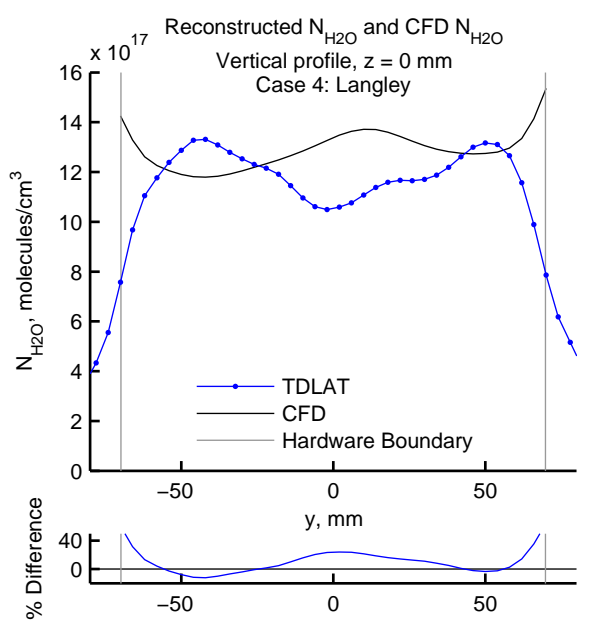

(c)

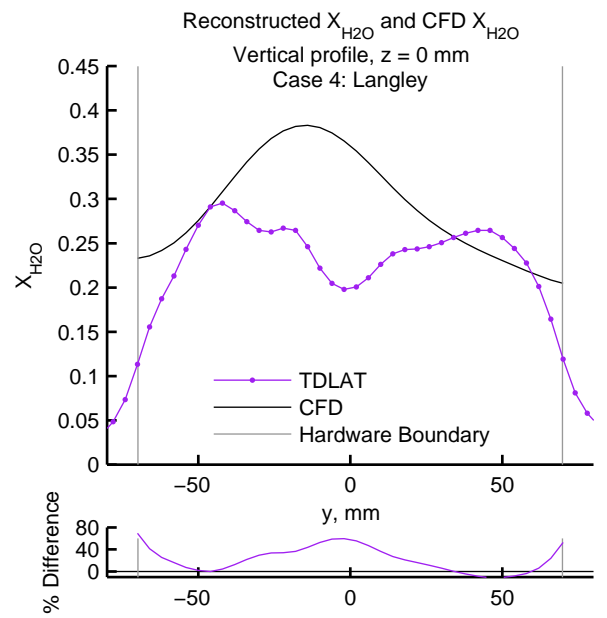

(e)

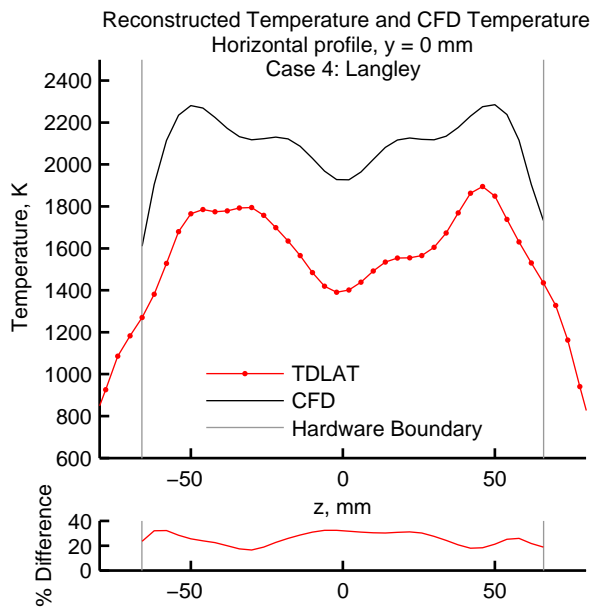

(b)

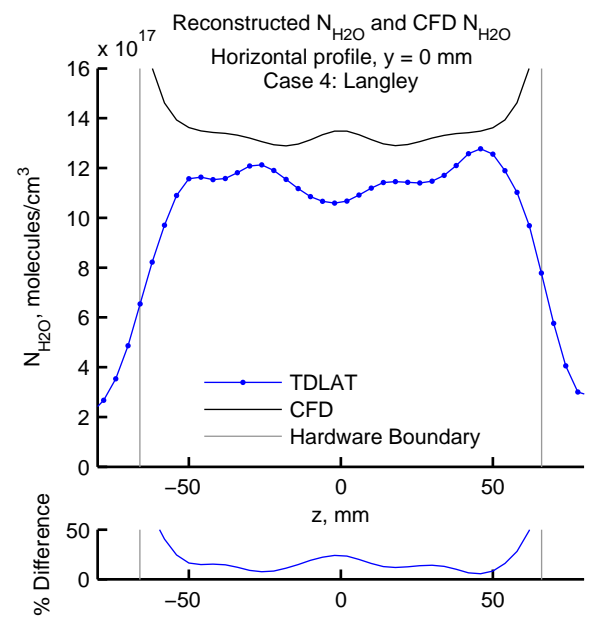

(d)

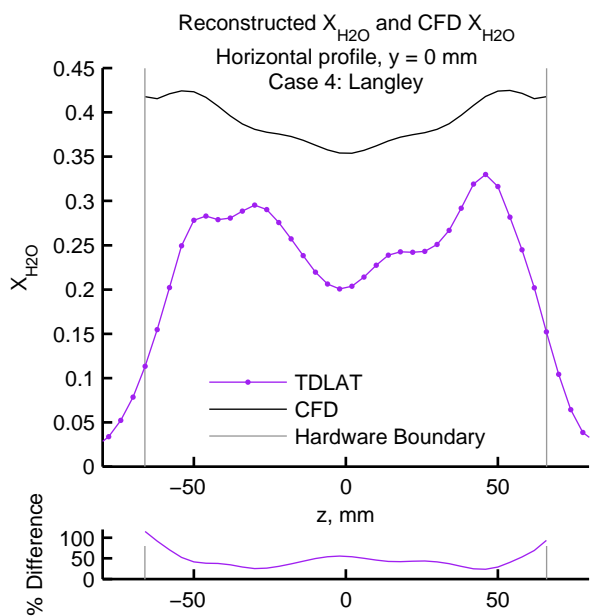

(f)

FiguRE 5.13: Line plot comparisons of TDLAT measurements and CFD at the exit plane of the NASA Langley DCSCTF for case 4, (a) vertical profiles for TDLAT and CFD temperatures, (b) horizontal profiles for TDLAT and CFD temperatures, (c) vertical profiles for TDLAT and CFD water vapor number densities, (d) horizontal profiles for TDLAT and CFD water vapor number densities, (e) vertical profiles for TDLAT and CFD water vapor mole fractions, and (f) horizontal profiles for TDLAT and CFD water vapor mole fractions. 


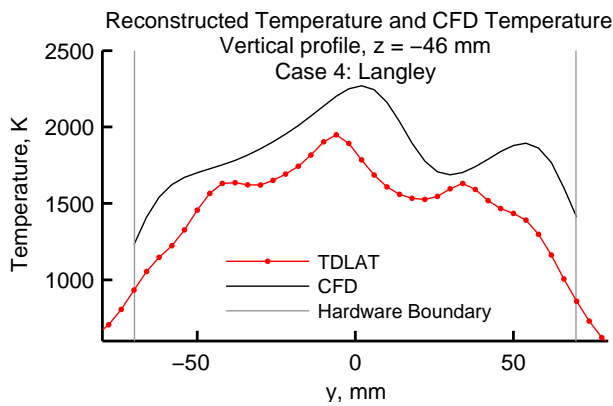

(a)

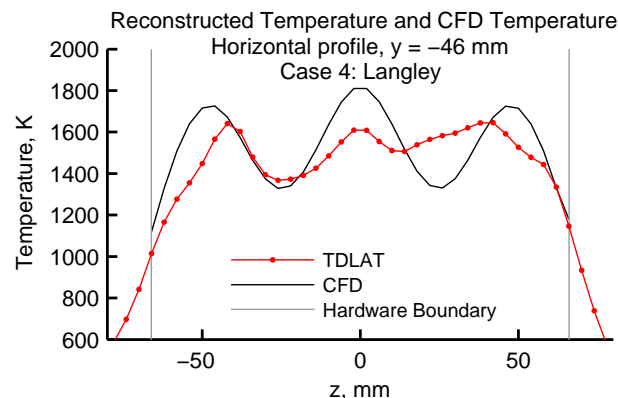

(c)

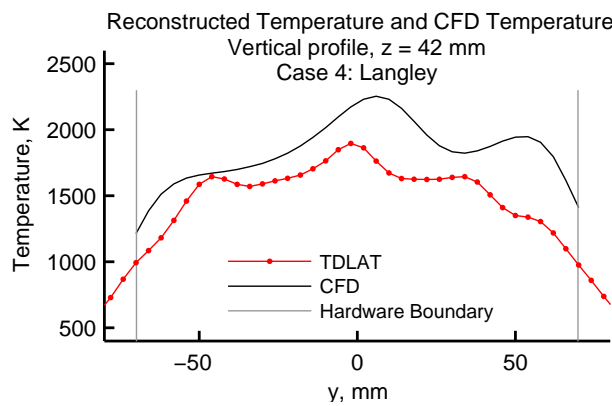

(b)

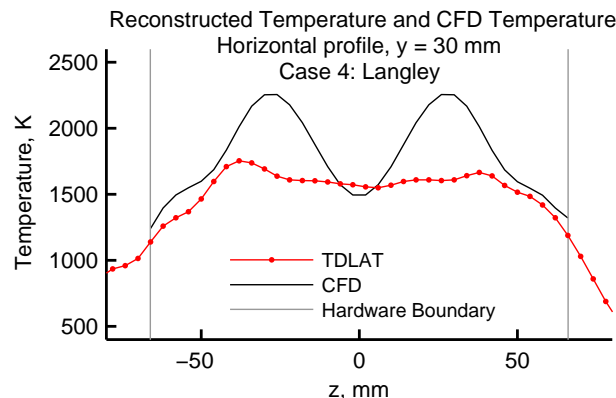

(d)

FiguRE 5.14: Off-center temperature line plot comparisons of TDLAT measurements and CFD at the exit plane of the NASA Langley DCSCTF for case 4.

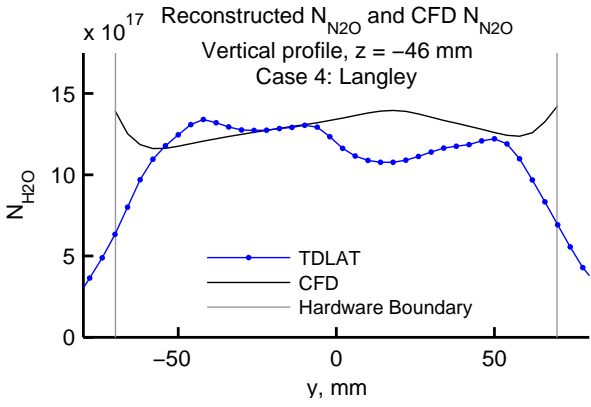

(a)

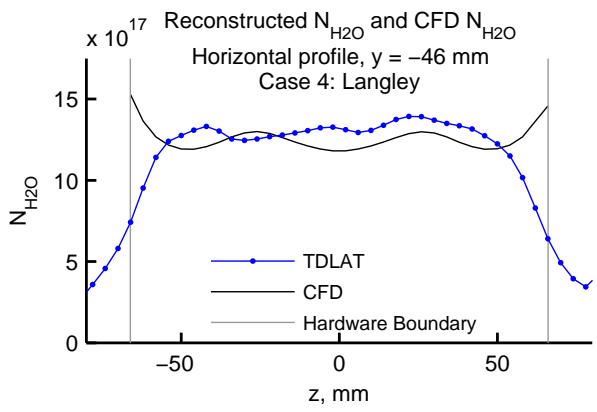

(c)

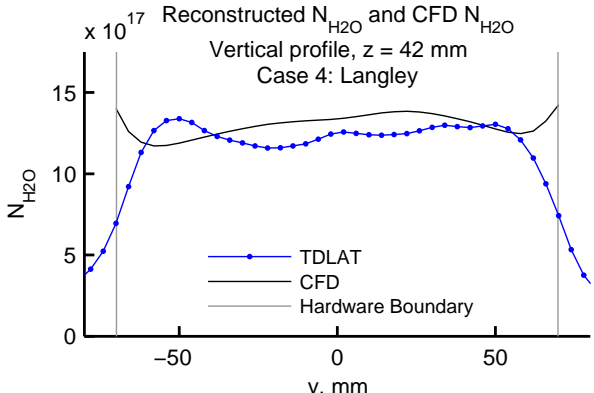

(b)

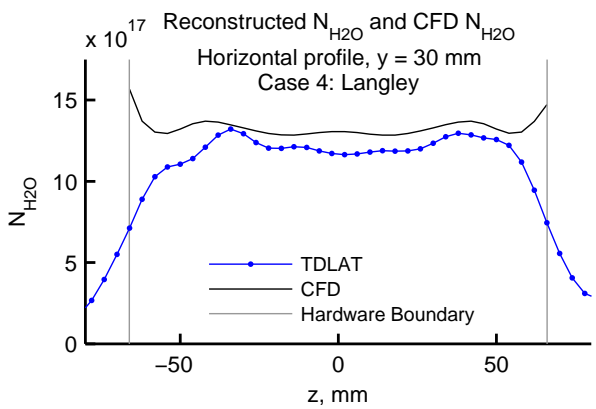

(d)

FIGURE 5.15: Off-center water vapor number density line plot comparisons of TDLAT measurements and CFD at the exit plane of the NASA Langley DCSCTF for case 4. 


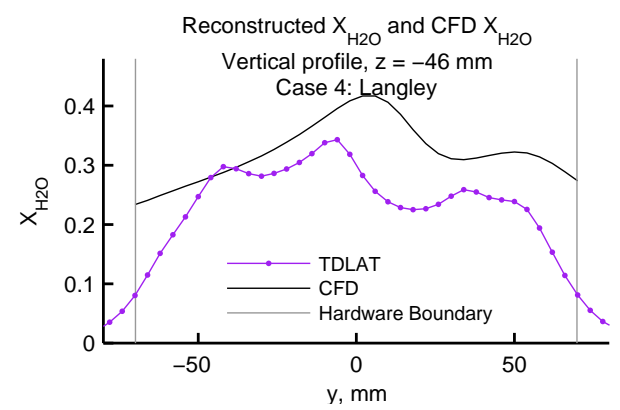

(a)

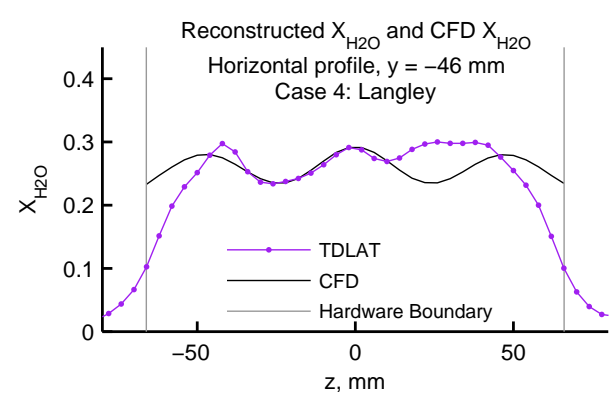

(c)

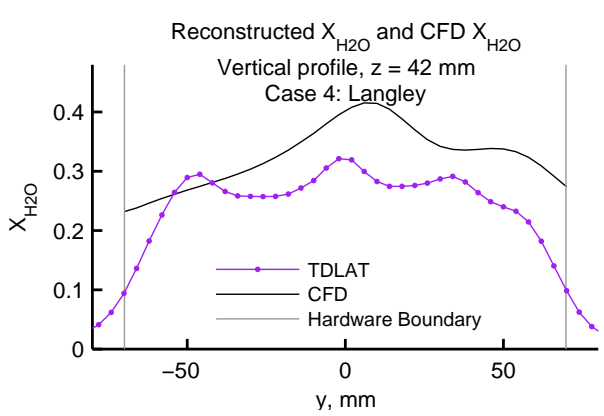

(b)

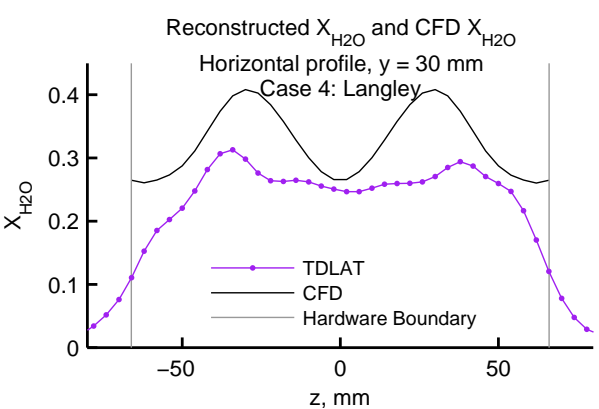

(d)

FiguRE 5.16: Off-center water vapor mole fraction line plot comparisons of TDLAT measurements and CFD at the exit plane of the NASA Langley DCSCTF for case 4.

From Figures 5.14, 5.15, and 5.16, good agreement is observed between the CFD simulations and TDLAT measurements. The temperature profiles show that the CFD predicts oscillations across the duct of a larger amplitude than are measured by TDLAT, especially along the z-direction. However, the TDLAT does capture very similar structure as predicted by the CFD, including the asymmetries in the vertical profiles, but the gradients are not as severe. The off-center water vapor number density line plots show good agreement between the CFD and TDLAT in magnitude. As was discussed previously, the CFD tends to have slightly larger values on average. Figure 5.16 shows several off-center line plots of the CFD calculated and TDLAT measured water vapor number density at the exit plane of the DCSCTF. As was seen for the temperature profile, the water vapor mole fraction profiles agree remarkably well. The structure predicted by the CFD, including asymmetries and oscillations, is captured by the TDLAT measurement. Again, the amplitude of the peak mole fractions measured by TDLAT are not quite as large as the CFD indicates. 


\section{Chapter 6}

\section{Summary and Future Work}

\subsection{Summary}

The work presented in this dissertation focused on the development of the optical diagnostic technique Tunable Diode Laser Absorption Tomography (TDLAT), and its application to two scramjet ground testing facilities. Building upon the work of previous students, Bryner and Snyder, the current work has matured the TDLAT technique both in the spectral analysis and tomographic processing codes, as well as in the design of the experiment itself. By combining the optical diagnostic technique Tunable Diode Laser Absorption Spectroscopy (TDLAS) with computed tomography algorithms, complex spatially-resolved thermodynamic quantities are able to be measured in the extraordinarily harsh conditions of (sc)ramjet exhaust flowfields. The TDLAT system has proven to be a valuable and unique diagnostic tool through measurements on both a dual-mode scramjet facility at the University of Virginia and a larger-scale direct-connect scramjet facility at NASA Langley Research Center.

The Research Objectives of this work, which were presented in Chapter 1 and are restated below for convenience, have each been addressed and the significance of each contribution is discussed below. 
I Develop efficient and accessible (well-documented) analysis codes for both spectral and tomographic reconstruction analysis

II Measure temperature and $\mathrm{H}_{2} \mathrm{O}$ number density distributions at the exit plane of the University of Virginia's Supersonic Combustion Facility (UVaSCF) at two operating points: scram-mode operation and ram-mode operation

III Measure combustion efficiency of the UVaSCF at the exit plane, in conjunction with SPIV measurements

IV Modify and improve first iteration of TDLAT hardware and software for extension of the TDLAT technique to other experimental facilities (NASA Langley Direct-Connect Supersonic Combustion Test Facility)

\subsubsection{TDLAT Hardware and Software Summary}

The TDLAT technique has been improved with respect to both the experimental design and the analysis codes, addressing Research Objective I. With regard to the hardware, the redesign of the optical components, i.e. the TED box, was most significant. By moving away from Thorlabs construction cubes, the design allowed for much improved flexibility in the locations of its components. The SNR of the measured signal was improved by the enhanced alignment capability. As was proposed by Bryner, a multiple-TED box configuration was designed and utilized for measurements at NASA Langley Research Center. This configuration provided the benefit of greatly reducing experimental test duration.

The current spectral analysis codes were fully re-developed for automated processing of several thousand TDLAS LOS. The codes process the recorded data to obtain the relative frequency of the light, perform a preliminary baseline fit of the absorption feature, simultaneously perform a Voigt fit and secondary baseline fit of the absorption feature, and calculate integrated absorbance. These integrated absorbance values, organized into sinograms, represent several hundred TDLAT LOS 
measurements. The sheer quantity of these LOS measurements, each acquired at a different spatial location, is quite remarkable and is a significant achievement of this work.

A large portion of the current work was devoted to the improvement of the tomographic processing algorithm. Due mainly to non-transparency of the previously utilized algorithm, MATLAB's filtered back-projection algorithm ifanbeam.m, a new algorithm was investigated. Also based on the recommendation of Bryner, the algebraic reconstruction method of Maximum-Likelihood Expectation-Maximization (ML-EM) was selected for reconstruction of TDLAT measurements. Development and coding of the algorithm was executed, providing the user with full control and monitoring of all properties in the algorithm, e.g. beam collection geometry, orientation, number of iterations, and convergence. The importance of the transparency of this algorithm was discernible during the investigation of imaging artifacts and background absorbance subtraction. Two numerical methods were developed to address the problem of background absorbance. The second, simpler method, showed better integration with experimental data and was utilized for the subsequent TDLAT measurements.

\subsubsection{UVa Measurements Summary}

TDLAT measurements were first collected at an un-fueled condition, with $12 \%$ steam injection far upstream of the UVaSCF test section. The TDLAT measurements showed a near-constant $12 \%$ water vapor mole fraction at the exit plane of the UVaSCF. An asymmetry in the temperature distribution at this plane agreed well with previous experimental diagnostics further upstream. This experiment sought to measure a quantity which was known for the facility and had been measured previously by other researchers via TDLAS. The TDLAT's ability to measure and spatially resolve this known quantity of water vapor gives a great deal of confidence in the accuracy of the results for the subsequent experiments, in which the expected 
distributions are not as well-established.

TDLAT measurements have been collected at the exit plane of the UVaSCF at both scram-mode and ram-mode operation conditions, addressing Research Objective II. These experiments mark the first time tomographic absorption measurements with high spatial fidelity have been collected at the exit plane of a scramjet during both modes of combustion. An un-swept ramp fuel-injector was utilized to inject $\mathrm{H}_{2}$ in the combustor section. Measurements of the scram-mode of operation showed maximum temperatures near 1,400 $\mathrm{K}$ and maximum water vapor mole fractions near 10\%. In support of the National Center for Hypersonic Combined Cycle Propulsion (NCHCCP), the TDLAT measurements were used to validate computational fluid dynamic (CFD) calculations by Edwards and Fulton of the same test conditions. While the magnitude of the temperature distributions (and consequently the water vapor mole fraction distributions) differed, the shapes of the distributions were in excellent agreement. The comparison between the water vapor number density distributions, calculated by CFD and measured by TDLAT, showed remarkable agreement. The agreement between the simulation and the experiment, highlighted by line plots of the water vapor number density, was within $5 \%$ for a majority of the duct. The penetration of the high-temperature high-water vapor mole fraction core of the flow was seen to be the same in both the CFD and TDLAT measurements.

TDLAT measurements collected at the exit plane of the UVaSCF in the rammode of combustion showed much higher temperatures and larger water vapor mole fractions, as was expected. Maximum temperatures reached nearly 2,200 K, while maximum water vapor mole fraction reached nearly $25 \%$ at certain locations across the duct. High temperatures and water vapor mole fractions extended much further across the duct than in the scramjet test case. The same asymmetry observed in the temperature distribution for the $12 \%$ steam injection case was again observed in this ram-mode case.

A significant consequence of these TDLAT measurements is the ability to directly 
measure the combustion efficiency of the (sc)ramjet engine, addressing Research Objective III. By combining the spatially-resolved distributions of water vapor number density obtained by TDLAT with spatially-resolved distributions of axial velocity obtained by stereoscopic particle image velocimetry (SPIV), the first direct measurement of combustion efficiency in a (sc)ramjet via a comparison of injected $\mathrm{H}_{2}$ mass flow to exhausted $\mathrm{H}_{2} \mathrm{O}$ mass flow is achieved. This ability to directly measure the combustion efficiency of a (sc)ramjet is an extremely powerful capability and is a major result of this current work. As many scramjet ground-test facilities are in need of alternative means of characterizing scramjet test articles, besides traditional methods like thrust stands, optical diagnostics which can survive harsh environments are becoming critical. With suitable optical access, the approach of directly measuring combustion efficiency by combining TDLAT and SPIV measurements has been shown to be successful.

\subsubsection{NASA Langley Measurements Summary}

The extension of the TDLAT technique for application of the NASA Langley DCSCTF was also a significant contribution of this work and addressed Research Objective IV. Originally conceptualized and designed for use with the UVaSCF, the implementation of the TDLAT technique to the NASA Langley DCSCTF presented several scaling challenges, both in physical size and in test duration. The experimental design was adjusted to include five optical sources, TED boxes, reducing the duration of the experiment significantly.

TDLAT measurements at the exit plane of the DCSCTF resulted in 2-D distributions of temperature, water vapor number density, and water vapor mole fraction for a fuel-to-air equivalence ratio of 0.5. Maximum temperatures observed in the flowfield neared 2,100 K, while maximum water vapor mole fractions neared $38 \%$. A significant amount of structure was observed in the temperature and water vapor mole fraction distributions, with two main lobes of high-temperature high-water 
vapor mole fraction seen. The distribution resembled an "M", which was also predicted by CFD calculations performed by Gaffney of NASA Langley Research Center. Overall, CFD predicted slightly higher temperatures, water vapor number densities, and water vapor mole fractions than were measured by TDLAT, but the contours of the distributions were remarkably similar. Best agreement was seen in line plots at off-center locations, aligned with the high-temperature lobes.

The successful extension of this TDLAT technique to the NASA Langley DCSCTF demonstrates TDLAT's viability as an optical diagnostic for larger-scale scramjet ground test facilities. Though faced with several challenges related to the scaling of the test facility, the adaptability and robustness of the TDLAT system was only improved.

\subsection{Future Work}

This section proposes several changes which could be integrated into the TDLAT technique for improvement in the future. First, as was detailed in Chapter 3, the use of absorption feature KB1 resulted in the challenge of background absorbance. Due to its spectroscopic parameters, significant absorption was detected outside of the main region of interest (the hardware geometry of the UVaSCF). This absorption feature was selected for use mainly due to its well-characterized spectroscopic parameters, along with its proximity to the other absorption features utilized. Therefore, two solutions are presented: continue to use the set of absorption features described herein and decrease the light's path length through ambient room air, or discard the KB1 absorption feature in favor of a transition less susceptible to ambient air moisture. Due to the strong absorbance of the KB1 transition and its high SNR, the first solution is recommended. An improved nitrogen or dry-air purge system would allow for significantly less room-air absorption to occur.

Another proposed improvement of the TDLAT technique, one which would require 
significant experimental design changes, is the randomization of the LOS geometry. As was discussed in Chapter 3, a significant amount of the imaging artifacts observed arise as a result of the symmetry of the beam collection geometry. This symmetry, along with an aliasing error, creates large ripples in the reconstructed image, coalescing to a low-valued ring which corresponds to the inscribed angle of the fanbeam. A brief study performed showed that the randomization of the LOS geometry, i.e. not fanbeam or parallel beam, dissipated the strong gradient seen earlier in the distribution of total laser path per pixel. Removing this large gradient in flow measurement density also removed the imaging artifacts, seen so predominantly in the KB1 reconstruction before background subtraction. This type of asymmetric beam sampling has been utilized by other researchers [46], though not with such a large number of optical samples.

One challenge that the TDLAT system will need to overcome for future work with large scale facilities is the duration of testing required. There are two main elements of the TDLAT technique which contribute to this obstacle: the amount of time required to collect a satisfactory number of samples at each location due to SNR issues and the amount of time needed for the hardware to physically rotate to its next measurement location.

The first issue which elongates experiment times stems from needing to collect several single-scan LOS at each measurement position to overcome a poor SNR. The TDLAS technique, which is used to collected each TDLAT measurement, utilizes direct absorption spectroscopy. This type of absorption spectroscopy can be very susceptible to noise, especially in the baseline fitting process, and therefore several LOS (150 were utilized in this work) are needed to assure accurate measurement of the absorption feature. A derivative of direct absorption spectroscopy, known as wavelength modulation spectroscopy (WMS), operates on the same principals of direct absorption spectroscopy but includes a high-frequency sinusoidal modulation, which is superimposed upon the original sawtooth wave. When the resulting absorption sig- 
nal is detected, the first and second harmonics of the signal, known as the $1 f$ and $2 f$ respectively, are also detected. This technique, known as first-harmonic-normalized wavelength-modulation spectroscopy with second-harmonic detection (WMS- $2 f / 1 f$ ), has been shown to greatly increase the SNR of the resulting signal. [28][64] Replacing the traditional direct absorption TDLAS measurement with WMS- $2 f / 1 f$ in the TDLAT technique may have great potential to improve the SNR enough that fewer single-scan measurements are needed at each measurement position, thereby significantly reducing the test time required.

Lastly, a large amount of the time spent during measurement acquisitions comes from the physical movement of the TDLAT hardware itself. To reduce this unnecessary wasted time, it is proposed that the mechanical motion of the TDLAT measurement system be eliminated all together. Instead of physically moving a single TED box between each measurement point, it is possible that several stationary points of optical access could utilized. As was shown in the TDLAT experiments conducted at NASA Langley Research Center, each diode laser supplies sufficient power to traverse a 40 inch path length, even when split into five separate beams. Also, the relatively low cost of a diode laser $(\sim \$ 1500)$ and their "off-the-shelf" availability makes it plausible that enough LOS could be achieved with no motion at all. This would greatly reduce the duration of the experiment and could lead to the possibility of a temporally- and spatially-resolved TDLAT measurement. Not only would eliminating the motion from the TDLAT system reduce required test duration, it would also serve to toughen the already robust measurement system by removing a potential source of complication. 


\section{Bibliography}

[1] J.C. McDaniel. National Center for Hypersonic Combined Cycle Propulsion, proposal. December 2008.

[2] D.R. Jenkins. X-15: Extending the Frontiers of Flight. CreateSpace Independent Publishing Platform, 2010.

[3] M.E. White. The national aerospace plane program and the APL role. Johns Hopkins APL Technical Digest, 13(1):218-229, Jan-Mar 1992.

[4] L. Schweikart. The Hypersonic Revolution: Case Studies in the History of Hypersonic Technology, volume III. Air Force History and Museums Program, 1998.

[5] M.K. Smart, N.E. Hass, and A. Paull. Flight data analysis of the HyShot 2 scramjet flight experiment. AIAA Journal, 44(10):2366-2375, October 2006.

[6] G. Norris. X-51 waverider achieves goal on final flight. Aviation Week, May 2 2013. http://aviationweek.com/defense/x-51a-waverider-achieves-goal-finalflight.

[7] M. Lewis. X-51 scrams into the future. Aerospace America, 48(9):26-31, October 2010.

[8] J.W. Canan. Breathing new hope into hypersonics. Aerospace, 45(11):26-31, November 2007.

[9] M. Wall. Air force's x-51a hypersonic scramjet makes record-breaking final flight. Space.com, May 03 2013. http://www.space.com/20967-air-force-x-51ahypersonic-scramjet.html.

[10] M.G. Allen. Diode laser absorption sensors for gas-dynamic and combustion flows. Measure, 9:545-562, 1998.

[11] R.K. Hanson, P.A. Kuntz, and C.H. Kruger. High-resolution spectroscopy of combustion gases using a tunable IR diode laser. Applied O, 16(8):2045-2048, 1977 .

[12] A.C. Eckbreth, editor. Recent advances in laser diagnostics for temperature and species concentration in combustion, number pp 1471-90. 18th Symposium (Int.) On Combustion, 1981. Pittsburgh, PA: The Combustion Institute. 
[13] M. Kimball-Linne, G. Kychakoff, and R.K. Hanson. Fiber-optic absorption/fluorescence combustion diagnostics. Combustion Science and Technology, 49:307-22, 1986.

[14] J.A. Silver and A.C. Stanton. Airborne measurement of humidity using a singlemode PB salt diode-laser. Applied Optics, 26(13):2558-2566, July 11987.

[15] L.S. Rothman, R.R. Gamache, L.R. Brown, R.A. Toth, H.M. Pickett, R.L. Poynter, J.-M. Flaud, C. Camy-Peyret, A. Barbe, N. Husson, C.P. Rinsland, and M.A.H. Smith. The HITRAN database: 1986 edition. Applied Optics, 26(19):4058-4097, October 1987.

[16] L.S. Rothman, I.E. Gordon, R.J. Barber, H. Dothe, R.R. Gamache, A. Goldman, V. Perevalov, S.A. Tashkun, and J. Tennyson. HITEMP, the high-temperature molecular spectroscopic database. Journal of Quantitative Spectroscopy \& Radiative Transfer, 111(15):2139-2150, 2010.

[17] V. Nagali and R.K. Hanson. Design of a diode-laser sensor to monitor water vapor in high-pressure combustion gases. Applied Optics, 36(36):9518-9527, Dec 201997.

[18] D.S. Baer, V. Nagali, E.R. Furlong, R.K. Hanson, and M.E. Newfield. Scannedand fixed-wavelength absorption diagnostics for combustion measurements using multiplexed diode lasers. AIAA Journal, 34(3):489-493, Mar 1996.

[19] M.E. Webber, J. Wang, S.T. Sanders, D.S. Baer, and R.K. Hanson, editors. In situ combustion measurements of $\mathrm{CO}, \mathrm{CO} 2, \mathrm{H} 2 \mathrm{O}$ and temperature using diode laser absorption sensors, volume 28. Proceedings of the Combustion Institute, 2000 .

[20] H. Teichert, T. Fernholz, and V. Ebert. Simultaneous in situ measurement of CO, $\mathrm{H} 2 \mathrm{O}$, and gas temperatures in a full-sized coal-fired power plant by near-infrared diode lasers. Applied Optics, 42(12):2043-2051, Apr 202003.

[21] L.C. Philippe and R.K. Hanson. Laser-absorption mass flux sensor for high-speed air-flows. Optics Letters, 16(24):2002-2004, Dec 151991.

[22] M.P. Arroyo, S. Langlois, and R.K. Hanson. Diode-laser absorption technique for simultaneous measurement of multiple gasdynamic parameters in high-speed flows containing water-vapor. Applied Optics, 33(15):3296-3307, May 201994.

[23] M.F. Miller, W.J. Kessler, and M.G. Allen. Diode laser-based air mass flux sensor for subsonic aeropropulsion inlets. Applied Optics, 35(24):4905-4912, Aug 201996.

[24] E.A. Whittaker, C.M. Shum, H. Grebel, and H. Lotem. Reduction of residual amplitude modulation in frequency-modulation spectroscopy by using harmonic frequency modulation. Journal of the Optical Society of America B, 5(6):12531256, June 1988. 
[25] D.S. Bomse, A.C. Stanton, and J.A. Silver. Frequency-modulation and wavelength modulation spectroscopies - comparison of experimental methods using a lead-salt diode-laser. Applied Optics, 31(6):718-731, February 201992.

[26] J.J. Nikkari, J.M. Di Iorio, and M.J. Thomson. In situ combustion measurement of $\mathrm{CO}, \mathrm{H} 2 \mathrm{O}$, and temperature with a 1.58-mu $\mathrm{m}$ diode laser and two-tone frequency modulation. Applied Optics, 41(3):446-452, January 202002.

[27] E.C. Rea, A.Y. Chang, and R.K. Hanson. Rapid laser wavelength modulation spectroscopy applied as a fast temperature measurement technique in hydrocarbon combustion. Applied Optics, 27(21):4454-4464, November 11988.

[28] C.S. Goldenstein, R.M. Spearrin, I.A. Schultz, J.B. Jeffries, and R.K. Hanson. Wavelength-modulation spectroscopy near $1.4 \mathrm{mu} \mathrm{m}$ for measurement of $\mathrm{H} 2 \mathrm{O}$ and temperature in high-pressure and -temperature gases. Measurement Science and Technology, 25(5):1-9, May 2014.

[29] JA Silver and DJ Kane. Diode laser measurements of concentration and temperature in microgravity combustion. Measurement Science and Technology, 10(10):845-852, Oct 1999.

[30] P. Kauranen, H.M. Hertz, and S. Svanberg. Tomographic imaging of fluidflows by the use of 2-tone frequency-modulation spectroscopy. Optics Letters, 19(18):1489-1491, Sep 151994.

[31] E. Bryner. Development of Tunable Diode Laser Absorption Tomography for Determination of Spatially Resolved Distributions of Water Vapor Temperature and Concentration. PhD thesis, University of Virginia, December 2010.

[32] F. Wang, K.F. Cen, N. Li, J.B. Jeffries, Q.X. Huang, J.H. Yan, and Y. Chi. Twodimensional tomography for gas concentration and temperature distributions based on tunable diode laser absorption spectroscopy. Measurement Science and Technology, 21:1-10, Feb 232010.

[33] M.S. Brown. Application of diode-laser-based measurements in hypersonic flows. Number AIAA 2012-0555, Nashville, Tennessee, January 9-12 2012. American Institute for Aeronautics and Astronautics Aerospaces Sciences Meeting.

[34] M.S. Brown, G.C. Herring, K. Cabell, N. Hass, T.F. Barhorst, and M. Gruber. Optical measurements at the combustor exit of the HIFiRE 2 ground test engine. Number AIAA 2012-0857, Nashville, Tennessee, January 9-12 2012. American Institute of Aeronautics and Astronautics Aerospace Sciences Meeting.

[35] Z. Nadir, M.S. Brown, M.L. Comer, and C.A. Bouman. Tomographic reconstruction of flow gases using sparse training. Paris, France, October 27-30 2014. IEEE International Conference on Image Processing. 
[36] C. Segal, J.C. McDaniel, R.H. Krauss, and R.B.III Whitehurst. Combustion efficiency determined from wall pressure and temperature measurement in a Mach 2 combustor. Number AIAA-91-0017, January 1991.

[37] M.S. Brown, S. Williams, C.D. Linstrom, and D.L. Barone. Progress in applying tunable diode laser absorption spectroscopy to scramjet isolators and combustors. Final Report AFRL-RZ-WP-TP-2010-2146, Propulsion Sciences Branch (AFRL/RZAS), Aerospace Propulsion Division, AifrForce Research Laboratory, Propulsion Directorate, United States Air Force, May 2010.

[38] A.C. Kak and Malcolm Slaney. Principles of computerized tomographic imaging, 2001.

[39] E.J. Hoffman, S. Huang, and M.E. Phelps. Quantitation in positron emission computed tomography: 1. effect of object size. Journal of Computer Assisted Tomography, 3:299-308, 1979.

[40] O. Medalia, I. Weber, A.S. Frangakis, D. Nicastro, G. Cerisch, and W. Baumeister. Macromolecular architecture in eukaryotic cells visualized by cryoelectron tomography. Science, 298(5596):1209, 2002.

[41] D. Lal. Cosmic ray labeling of erosion surfaces: in situ nuclide production rates and erosion models. Earth and Planetary Science Letters, 104:424-439, 1991.

[42] G.N. Hounsfield. Computerized transverse axial scanning (tomography): Part I. description of system. British Journal of Radiology, 46:1016-1022, 1973.

[43] K.M. Busa, E. Bryner, J.C. McDaniel, C.P. Goyne, G.S. Diskin, and C.T. Smith. Demonstration of capability of water flux measurement in a scramjet combustor using Tunable Diode Laser Absorption Tomography and Stereoscopic PIV. Number AIAA-2011-1294, Jan 2011.

[44] E. Bryner, M.G. Sharma, C.P. Goyne, M.C. Snyder, R.H. Krauss, and E.F. Martin. Tunable Diode Laser Absorption technique development for determination of spatially resolved water concentration and temperature. Number AIAA-2010299, Jan 2010.

[45] R. Gordon, R. Bender, and G.T. Herman. Algebraic reconstruction technique (ART) for three-dimensional electron microscopy and X-ray photography. Journal of Theoretical Biology, 29:471-481, 1970.

[46] W. Verkruysse and L.A. Todd. Novel algorithm for tomographic reconstruction of atmospheric chemicals with sparse sampling. Environmental Science and Technology, 39:2247-2254, 2005.

[47] X. Zhou, J.B. Jeffries, and R.K. Hanson. Development of a fast temperature sensor for combustion gases using a single tunable diode laser. Applied Physics $B, 81: 711-722,2005$. 
[48] C.S. Goldenstein, I.A. Schultz, J.B. Jeffries, and R.K. Hanson. Tunable diode laser absorption sensor for measurement of temperature and water concentration in supersonic flows. Number AIAA 2011-1094, Orlando, FL, January 4 - 72011. 49th AIAA Aerospace Sciences Meeting.

[49] M.C. Snyder. Development of a tunable diode laser absorption tomography technique. Master's thesis, University of Virginia, January 2009.

[50] F.L. Pedrotti, L.S. Pedrotti, and L.M. Pedrotti. Introduction to Optics. Pearson Education, Inc., 3 edition, 2007.

[51] D.L. Barone. Investigation of TDLAS measurement in a scramjet engine. Master's thesis, University of Cincinnati, May 212010.

[52] E.E. Whiting. An emperical approximation to the Voigt profile. Journal of Quantitative Spectroscopy \& Radiative Transfer, 8:1379-1384, 1968.

[53] S.M. Abrarov and B.M. Quine. Efficient algorithm implementation of the Voigt/complex error function based on exponential series approximation. Applied Mathematics and Computation, 2011.

[54] M. Kuntz. A new implementation of the Humlicek algorithm for the calculation of the Voigt profile function. Journal of Quantitative Spectroscopy 85 Radiative Transfer, 57(6):819-824, 1997.

[55] J.A. Fulton, J.R. Edwards, H.A. Hassan, J.C. McDaniel, C. P. Goyne, R.D. Rockwell, A. Cutler, E.C.A. Gallo, and L.M.L. Cantu. Continued hybrid LES/RANS simulation of a hypersonic dual-mode scramjet combustor. Number AIAA 20130117, Grapevine, Texas, January 7-10 2013. AIAA Aerospace Sciences Meeting.

[56] W.H. Press, S.A. Teukolsky, W.T. Vetterling, and B.P. Flannery. Numerical Recipes in $\mathrm{C}++$. Cambridge Univresity Press, Cambridge, England, 2002. Chap. 15.

[57] R.D. Rockwell, C.P. Goyne, W. Haw, J.C. McDaniel, C.S. Goldenstein, I.A. Schultz, J.B. Jeffries, and R.K. Hanson. Measurement of water vapor levels for investigating vitiation effect of scramjet performance. Journal of Propulsion and Power, 27(6):1315-1317, November-December 2011.

[58] A.D. Cutler, G. Magnotti, L. Cantu, E. Gallo, P.M. Danehy, R. Rockwell, C. Goyne, and J. McDaniel. Dual-pump CARS measurement in the University of Virginia's dual-mode scramjet: Configuration "C". Number AIAA 2013-0335. AIAA Aerospace Sciences Meeting, January 7-10 2013.

[59] M.P. Burke, M. Chaos, Y. Ju, F.L. Dryer, and S.J. Klippenstein. Comprehensive $\mathrm{H} 2 / \mathrm{O} 2$ kinetic model for high-pressure combustion. International Journal of Chemical Kinetics, 44(7):444-474, 2012. 
[60] B.E. Rice. Characterization of a Dual-Mode Scramjet via Stereoscopic Particle Image Velocimetry. PhD thesis, University of Virginia, August 2014.

[61] B.E. Rice, C.P. Goyne, J.C. McDaniel, and R.D. Rockwell Jr. Characterization of a dual-mode scramjet via stereoscopic particle image velocimetry. Number 2014-0986. AIAA Aerospace Sciences Meeting, January 13-17 2014.

[62] NASA LaRC. The Langley Direct-Connect supersonic combustion test facility brochure, October 2005.

[63] R. Gaffney. Personal communication. NASA Langley Research Center, Hypersonic Airbreathing Propulsion Branch, Hampton, VA. November 2012.

[64] J.T.C. Liu, J.B. Jeffries, and R.K. Hanson. Wavelength modulation absorption spectroscopy with $2 \mathrm{f}$ detection using multiplexed diode lasers for rapid temperature measurements in gaseous flows. Applied Physics B, 78:503-511, 2004. 